\title{
FLORI FOSSILIS
}

\section{FOR IIITIONIS OOLITHICAE}

\author{
LE \\ PIAUTE F0SSILI DELL' 00LITE
}

\author{
DESCRITTE ED ILLUSTRATE
}

\author{
DAL \\ BARONE ACIIILLE DE ZIGIO
}

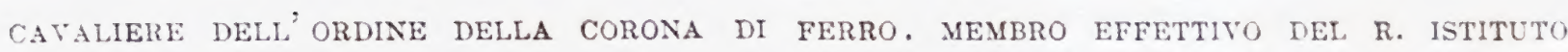
veNeto dI SCIENZE LETTERE ED ARTI, DELL' ACCADEMIA DI SCIENZE LETTERE ED ARTI II padova, della società italiana delle scienze naturali di milano, delia società

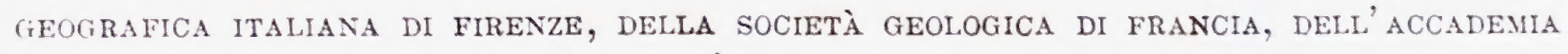
mp. Leopold. Carol. Dei naturalisti d'alemagna, della società imp. dei naturalisti

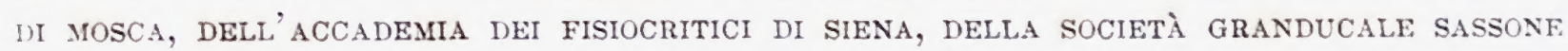
Di mineralogia, Geologia e paleontologia in Jena. membro onorario dell' aCCAdema di verona, dell' ateneo di treviso, della società di Storia Naturale di dresda E di

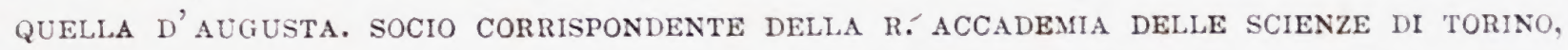

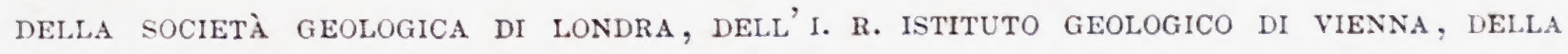

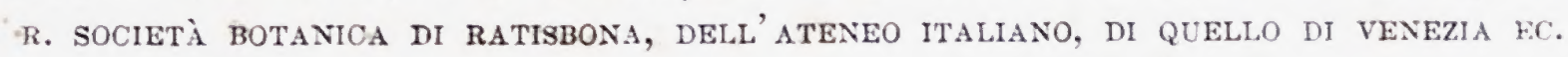

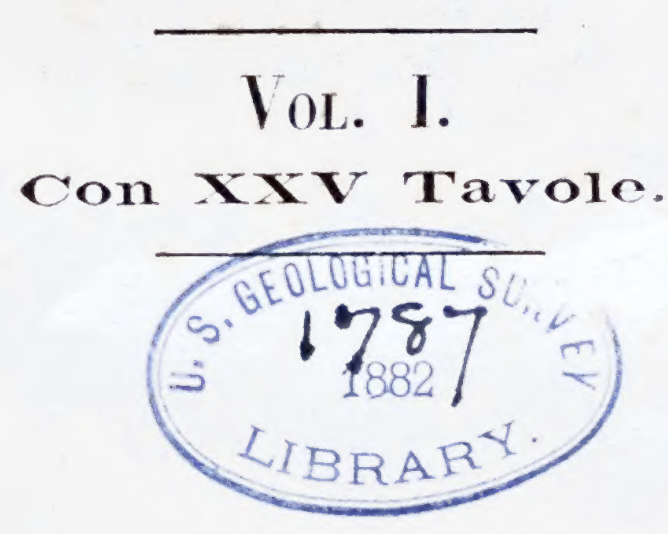

PA D O V A

DALLA TIPOGRAFIA DEL SEMINARIO 

Se lo studio dei fossili animali contribui a spargere molta luce sulla storia antica del globo, non meno importante dee risguardarsi quello delle piante che stanno sepolte negli strati delle diverse formazioni. Imperciocchè dai resti vegetali in cui sia dato riconoscere se abbiano appartenuto a piante o terrestri, o d'acqua dolce, o marine, siamo tratti a considerare quali fossero i limiti e la natura delle terre emerse nelle epoche più remote; ed i confronti fra le spente Flore e quelle che ora rivestono le varie zone del suolo che ci sopporta, aprono vasto campo alla investigazione delle condizioni che favorirono di preferenza lo svolgimento di certe famiglie ed improntarono di caratteri speciali la vegetazione dei diversi periodi geologici.

Quindi alla miglior conoscenza delle vicende cui soggiacque la corteccia terrestre non è, di altri studj a ciò intesi, meno giovevole quello delle piante fossili, ed ha già chiarito, per opera di valenti osservatori, buon numero di fatti importantissimi tanto per la scienza, quanto per le sue applicazioni.

E per vero, se la investigazione delle piante fossili, diretta a stenebrare il bujo in cui s'avvolge la Geografia di que' tempi che parlano alla nostra immaginazione un linguaggio si misterioso e solenne, tende a soddisfare l'irrequieta brama di sapere che ci tormenta; la esatta cognizione delle specie giova a farci riconoscere quali sieno $\mathrm{i}$ depositi di combustibile fossile che ponno tornare opportuni ad appagare $\mathrm{i}$ bisogni delle molteplici industrie, onde il genio inventivo del secolo presente va giustamente superbo.

Questa fatta di ricerche, sebbene avesse eccitata la curiosita dei nostri avi fino dai tempi del Listero, del Luidio e dello Schenzero, non poteva però estendersi e riuscire proficua nelle sue applicazioni se non se proce- 
dendo di pari passo col progresso di quella scienza la quale si fece guida sicura all' arte delle miniere, e che ajutata dalla Botanica e dalla Zoologia giunse a svelarci le prime orme di vita nelle varie forme delle spoglie organiche impietrite. Perciò veggiamo soltanto negli ultimi cinquant' anni e dopo che si segnarono con maggior precisione i limiti delle formazioni geologiche ed i caratteri delle singole Faune che le distinguono, moltiplicarsi le opere indirizzate ad investigare la natura della vegetazione delle diverse epoche geologiche, mercè il diligente studio dei resti fossili vegetali che si andavano scoprendo nei varii piani della serie cronologica dei terreni.

Tra le numerose e svariate pubblicazioni di cui s'arrichi la scienza in questo lasso di tempo, tengono a buon dritto il primo luogo, pella vastità dell'impresa, le Opere intente a schierarci dinanzi le descrizioni metodicamente ordinate di tutte le piante fossili conosciute. La Histoire des Végétaux fossiles ed il Tableau des Genres des Végétaux fossiles dell'illustre Brongniart, il Versuch einer Geognostisch Botanischen Darstellung der Flora der Vorwelt del Conte di Sternberg e l'Opera del chiarissimo Prof. Unger intitolata, Genera et Species Plantarum fossilium ci offrono splendidi esempi di un tal genere di lavori. È però a deplorarsi come la Histoire des Végétaux fossiles sia rimasta interrotta dopo la pubblicazione delle prime puntate del secondo Volume, e come l'Opera dello Sternberg manchi di un indice generale.

Meno precoce imprendimento e più fruttuoso nello stato attuale della scienza è senza dubbio quello delle Monografie e delle Flore speciali, che stringendo la cerchia delle osservazioni ad un campo più limitato, preparano con diligente studio i materiali per una futura più esatta compilazione delle Opere generali.

Stimo impertanto che i lavori più acconci a far rapidamente progredire questo ramo di scienza sieno: $1 .^{\circ}$ le Monografie di singole Classi o Famiglie che ne svelano la storia seguendone le spoglie in tutte le formazioni, in cui si trovano sepolte; $2 .{ }^{\circ}$ le Flore locali che porgono la illustrazione di tutte le specie rinvenute in una o più formazioni di una data regione; $\mathbf{3}^{\circ}$ le Flore che sebbene limitate alla illustrazione delle piante vissute durante un solo periodo geologico, spaziano però con ampiezza di ac- 


\section{$-\mathrm{V}-$}

curate indagini per tulto il globo e ci danno bello e compiuto uno dei piani che dovrà prender posto, quando che sia, nell' edificio di una Paleofitologia universale.

A quest'ultima categoria spetta l'Opera presente, che ha per iscopo la descrizione particolareggiata delle piante fossili fin qui trovate nei diversi piani della formazione oolitica. Da più anni rivolsi ogni mia cura a questo lavoro, incoraggiato dalla favorevole circostanza che le Alpi Venete mi avevano dischiuso nelle roccie di quel periodo, un' abbondante messe di resti vegetali, pel cui esame dovetti necessariamente svolgere le pagine di quanto era stato altrove pubblicato sulle piante dell' oolite, e ciò mi convinse della utilità che avrebbe recato ai cultori di questo ramo, non solo la illustrazione delle specie nuove trovate in questa parte d'Italia, ma eziandio una diligente revisione di tutte quelle che si erano rinvenute in altri paesi nella formazione medesima. E come suole avvenire che dall'unione di molti fatti scaturiscano quasi di per sè alcuni principj generali; cosi, dallo studio di tutto che fu scritto sulla Flora dell'Oolite e da quello dei copiosi materiali che potei adunare, mi fu aperta la via a rendere di pubblico diritto alcune considerazioni sulla distribuzione geografica e geologica di questa Flora, sulle famiglie che la compongono e sulle analogie che presenta paragonata colle Flore delle formazioni vicine e coll'attuale vegetazione di alcune regioni.

Queste considerazioni formarono il subbietlo di una Memoria sulla Flora dell oolite che lessi all' Istituto Veneto di Scienze Lettere ed Arti nel Marzo 1836, e che fu inserita nel Vol. VI. delle Memorie di quel Corpo Scientifico, nella quale fra le altre cose, parlando della estensione geografica di questa Flora ed annoverando tutti i punti del globo in cui era stata citata la presenza di piante nell'Oolite, esprimeva il dubbio che fosse ancor problematica la spettanza alla serie oolitica dei resti vegetali scoperti nella Virginia Orientale, nella penisola Indiana, nell' Australia e nella Tasmania, comunque parecchi distinti geologi si mostrassero in allora propensi a collocarli in quella formazione.

Tuttavia l'autorevole parere di quest'ultimi mi persuase ad annoverare anche le specie proprie di que' depositi nelle prime puntate del presente 
lavoro che si stamparono negli anni 1836 e 1838. Se non che avendo alcune circostanze, indipendenti dalla mia volonta, cagionato un ritardo di più anni alla continuazione di questa Opera, le osservazioni che si fecero in questo mezzo tempo contribuirono a far escludere del tulto dall' oolite alcuni di questi depositi fitolitiferi e risvegliarono nuove incertezze sull' appartenenza degli altri a questa formazione $\left(^{*}\right)$.

Contemporaneamente il chiarissimo Prof. Oldham di Calculta scopriva la magnifica Flora di Rajmahal nelle Indie, e il Sig. R. Tate illustrava le piante fossili scoperte nei terreni giurassici di Uitenhage nell'Africa meridionale, ma queste due Flore, che probabilmente appartengono ad alcuno dei piani inferiori del sistema giurassico, non ci porgono ancora dati sufficienti per decidere se spettino al Lias od all'Oolite inferiore. Perciò ho creduto che tornar potesse di maggiore utilità agli studiosi, il limitare la presente Opera alla accurata illustrazione di quelle sole specie che si rinvennero in terreni riconosciuti generalmente appartenere con certezza alla serie oolitica, e per tal guisa riunendo come in un quadro tutte le forme indubbiamente spettanti a questa formazione, porgere un'idea più precisa della vegetazione di quel periodo geologico, e somministrare elementi più positivi cui raffrontare le piante scoperte in altre regioni in terreni non ancor ben definiti, e che lasciano aperto il campo a dubitare se debbano appartenervi.

Dopo queste premesse è forza convenire che i depositi con piante fossili riconosciuti decisamente proprii dell' oolite, si limiterebbero per ora a quelli rinvenuti in Europa, e fra questi i più importanti e più ricchi di specie son senza dubbio quelli dell' Inghilterra, ove da gran tempo suonarono celebri per queste piante i terreni oolitici delle vicinanze di Whilby e di Scarborough nella Contea di York. Sembra che i Sig. Young e Bird sieno stati i primi a richiamare l'altenzione del pubblico su questi resti vegetali, porgendone alcuni delineati nel loro Geological Surcey of the

(*) Zıgno, Sopra i depositi di Piante fossili dell'America seltentrionale delle Indie e dell'Australia che alcuni Autori riferirono all' epoca Oolitica. Ris. Period. 1. R. Accad. Pad. 1863, pag. 139. 
Yorkshire Coast stampato nel 1822. Il Prof. Phillips nel 1835 ne annoverò trentatre specie, che figurò, ma non descrisse, nella sua Opera intitolata $\mathrm{Il}$ lustrations of the Geology of Yorkshire. Nella Fossil Flora of Great Britain dei Signori Lindley e Hutton ne troviamo cinquantasette specie, e questo numero s'accrebbe fino alle sessantatre nelle opere del Brongniart ed alle ottantatre in quella dello Sternberg. Non poche altre specie si scopersero di poi, e fra queste meritano speciale menzione quelle illustrate da Sir C. Fox Bunbury, Bart. nella sua dotta scrittura intitolata, On some Fossil Plants from the Jurassic strata of the Yorkshire coast, inserita alla pag. 179 del Vol. VII. del Quarterly Journal of the Geological Society of London, 1831. Per le quali scoperte noi veggiamo il novero delle piante fossili trovate nella formazione oolitica d'Inghilterra giungere nel 1834 a ben cento tredici specie, come si può rilevare dalla seconda edizione del Catalogue of British Fossils del Prof. Morris. Dopo quell' epoca la Flora Oolitica d'Inghilterra s'arricchi di parecchie altre forme illustrate dal chiarissimo Sig. Leckenby nel $\mathbf{1 8 6 5}$ in una Memoria inserita nel Vol. XX. del Quarterly Journal della Società Geologica di Londra, ove trattando dei terreni oolitici di Scarborough aggiunge la descrizione e le figure di alcune nuove specie di piante fossili e l' esame critico di altre già note.

Ma non è la sola Contea di York che contribui ad aumentare in questa guisa il numero delle piante oolitiche dell' Inghilterra, poichè i varii piani della formazione oolitica delle Contee di Dorset, di Oxford, di Gloucester e di Wilts, sebbene assai più scarsi di queste spoglie, ne porsero però anch' essi buona parte, come vedremo nelle considerazioni generali che serviranno di conclusione a quest' Opera.

Volgendoci verso Settentrione lungo il lido occidentale della Scozia, ci si affaccia l'isola d'Eig nelle cui stratificazioni, ritenute appartenere alla parte superiore della serie oolitica, scoperse il Witham dei frammenti, che esaminati al microscopio, gli diedero a divedere la struttura propria di una Conifera, alla quale impose il nome di Pinites Eggensis. Dal lato opposto, sulla riviera orientale della Scozia bagnata dal Mare del Nord, i banchi di combustibile fossile di Brora, adeguati dalla maestra penna di Sir R. I. Murchison, Bart. a quelli dell' oolite della Contea di York e le piante scoperte 
dal compianto Ugo Miller nei terreni oolitici dei contorni di Helmsdale nella Contea di Sutherland, segnano l'estremo punto in cui sieno comparse le traccie della Flora oolitica nell' emisfero boreale, fissandolo al grado $\mathbf{3 8} .^{\text {mo }}$ di latitudine settentrionale.

Nella Francia i terreni oolitici della Normandia presentano una serie corrispondente a quella che sta loro schierata dinanzi nell' Inghilterra meridionale, e si prolungano fino alla Loira formando cosi il lembo occidentale della gran cerchia giurassica, che circoscrive i bacini cretacei e terziarj di Londra e di Parigi.

Nel 1823 il Sig. J. Desnoyers scopriva su questa linea gli strati a piante fossili dei contorni di Mamers nel Dipartimento della Sarthe. Sono dessi composti di calcari bianchi a struttura oolitica, che egli adegua ai calcari scissili di Stonesfield e contengono quelle piante, che studiate dall'illustre Brongniart, si veggono descritte e figurate nel fascicolo d'aprile dello stesso anno negli Annales des Sciences Naturelles. Ma quelle determinazioni furono poscia reltificate dall'autore nelle sue opere posteriori, avendo riconosciuto come la maggior parte delle forme vegetali che aveva riferito alle Felci spettassero invece alle Cicadeacee.

Nella Francia settentrionale altri depositi filolitiferi si rinvennero poscia nell' oolite di Valoque e d'Alençon, poco lungi da Mamers ed in quella di Verdun, di Vaucouleurs e di S. ${ }^{t}$ Michel nel Dipartimento della Meuse. Nella Francia centrale, i contorni di Chatillon sur Seine nel Dipartimento della Côte d'or, e quelli di Châteauroux, fornirono pure alcuni bei saggi di Felci e di Cicadeacee, ed in maggior copia ne vediamo provveduti i terreni oolitici che si protendono verso Sud-Est, ad Orbagnoux, Abergement e Seyssel nel Dipartimento dell'Ain, ed in quelli delle vicinanze di Morestel nel Dipartimento dell'Isère. Questi depositi non appartengono però tulti allo stesso orizzonte, come vedremmo nell'ultima parte di quest' Opera, ove tratterò della distribuzione geografica e geologica della Flora dell' oolite.

Oltre ai luoghi testè indicati, altri se ne scopersero recentemente in Francia, ove l'oolite contiene piante fossili, cioè la Dorche presso Chanay nel Dipartimento dell' Ain, Ancy le Franc in quello dell'Yonne ed Etrochey presso Chatillon sur Seine nel Dipartimento della Côte d'or. Di quelle 
di la Dorche troviamo un breve cenno nell' Opera dell' illustre Prof. Heer intitolata, Urwelt der Schweiz pag. 145. Intorno a quelle degli altri depositi testè nominati si sta occupando il Conte Gastone di Saporta.

Anche la Svizzera forni non è guari il suo contingente alla Flora dell'oolite coi resti di Conifere e di Cicadee che si trovarono nel terreno Kimmeridiano di Vouargnez nel Vodese, nel coralliano di Olten e del Mont Risoux, e nell' Oxfordiano di Dänikon e di Bubendorf nel Cantone di Basilea.

Nell'Alemagna sono da gran tempo conosciuti i depositi di piante fossili di Nussplingen e Schnaithaim nel Wurtemberg, e di Solenhofen, Monheim e Kellheim nella Baviera, ove è da notarsi come alle piante terrestri siano associate le Alghe in numero prevalente. Questi depositi che si erano finora classificati quali rappresentanti di una zona equivalente al piano coralliano sono ora collocati dai Geologi Alemanni nella parte superiore del sistema oolitico. All'incontro i depositi fitolitiferi della Slesia e della Polonia che si credevano appartenere allo stesso orizzonte di quelli della Contea di York, per le recenti osservazioni del Roemer dovrebbero prender posto nella parte superiore del Keuper. Più verso settentrione i terreni a fossili oolitici di Kamenka e di Petrowekaya presso Izoume, di Popilani lungo il fiume Windau, dei contorni di Mosca e di qualche altro punto della Russia hanno pure svelato alcuni resti di Felci, di Cicadeacee e di Conifere giacenti in un orizzonte ancora non bene precisato, ma che sembra appartenere, o alla parte più bassa dell'oolite media, od alla serie oolitica inferiore.

Retrocedendo verso le regioni meridionali di Europa che cingono il gran bacino mediterraneo, noi c'imbatliano nei depositi delle Alpi Venete, ove assai prima di tutte le accennate scoperte si erano rinvenuti fino dal secolo scorso bellissimi esemplari di piante fossili presso il villaggio di Rolzo nei Sette Comuni del Vicentino in una roccia, la quale, come diremo in seguito, spetta senza alcun dubbio alla formazione oolitica. Se non che in quel tempo la geologia stratigrafica e paleontologica era ancor poco conosciuta, e s'ignoravano i caratteri distintivi delle diverse formazioni componenti le montagne. Perciò non dee recar meraviglia se a questa scoperta non si diede l'importanza che meritava, e se i naturalisti d'allora si limitarono a 
conservare alcuni saggi di queste piante nelle loro collezioni, come oggetti di semplice curiosità.

L'Abate Agostino dal Pozzo, nelle sue Memorie Istoriche dei Setle Comuni Vicentini narra come avvenisse il ritrovamento di queste piante nel 1764, nell'occasione che si scavavano delle lastre della pietra grigia di Rotzo per coprire i muricciuoli che attorniano il cimitero di quel Villaggio. Venti e più anni dopo tale scoperta, il Dottore Orazio Maria Pagani in una sua Orazione letta all'Accademia Olimpica di Vicenza nell' anno 1788, toccava egli pure questo argomento parlando di Rotzo e dicendo: "Ella è una cosa che "sorprende veramente e supera l'intelligenza, lo scoprire dentro a pietre "calcari finissime, compatte, rappresentazioni al naturale di piante e di erbe, "non mai conosciute dai più valenti botanici".

Reca, a dir vero, meraviglia come Giovanni Arduino, che poneva tanto studio nell' investigazione dei fenomeni geologici della sua patria, non abbia rivolta la sua attenzione a questo fatto che in quel torno aveva menato rumore. Di lui troviamo soltanto il seguente breve cenno, scritto due anni dopo l'Orazione del Pagani. "In Rotzo si trovano bensi entro dure pietre " calcaree a strati, delle piante solo indigene di climi caldissimi, dell' India " ec. " (Nuovo Giornale d'Italia 1790. Tomo I. pag. 552 nella Nota). Egualmente quel chiaro ingegno di G. B. Brocchi in una lettera al Gaidoni inserita l'anno 1794 nello stesso Giornale alla pag. 345 del Tomo V. dopo di avere parlato del famoso teschio di Coccodrillo, trovato a Treschè, si limita ad aggiungere in proposito le seguenti parole: "Eppure anche que"sta è una produzione non indigena dei nostri paesi, come non lo sono "alcune erbe esotiche, che si ritrovano in una pietra calcarea cenerognola "dei monti di Rotzo nei Selte Comuni".

Si trattenne più a lungo sopra questo interessante argomento il celebre Abate Fortis, il quale nelle sue, Mémoires pour servir a l'Histoire naturelle et principalement a l'Oryctographie de l'Italie et des pays adjacens, stampate a Parigi nel 1802, parlando dei luoghi più rimarchevoli dei Sette Comuni, alla pag. 92 del Vol. 11. cosi si esprime: "Je n'ai visité la haut "d'autre localitè interessante, que celle de Rotzo; ou dans une couche de "pierre calcaire bleuâtre et assez compacte on trouve des plantes desse- 
" chées et conservées dans leur état naturel, sans étre aucunement incorpo"rées avec la substance pierreuse qui les renferme. Lorsque on en rencon"tre, en cassant la pierre on peut en détacher les feuilles aussi aisement, "que si elles étaient prises entre deux cartons". E più innanzi nello stesso Volume alla pag. 158 ripete questa indicazione, accennando alla inclinazione degli strati di Rotzo e dicendo: "Les couches calcaires de prés de "Rotzo, sont trés inclinées vers le sud. A deux on trois cents pas de l'Egli"se paroissiale de Rotzo, tout a coté du chemin, on voit une de ces cou" ches de couleur bleuâtre, dans laquelle se trouvent, quoique bien rare"ment, conservées dans leur état naturel comme dans un herbier, au des"sêchement près, quelques fougères éxoliques dont les originaux sont en"core inconnus. On nous a même assuré qu'on y a rencontré quelques "squeleltes de poissons".

Nulla di più ci lasciò scritto questo arguto indagatore dei fenomeni geologici, sopra un subbietto cosi importante, e duole ch' egli non abbia spinto le sue ricerche allo scopo di conoscere, ove si conservassero quegli scheletri di pesci, che gli si dicevano occorsi a taluno in mezzo alla roccia medesima, in cui si trovano rapprese le piante. Intorno a questi ittioliti non s'ebbero dipoi altre notizie, ma che vi esistano è sufficientemente provato dai denti di Pycnodus che osservai negli strali di Rotzo e sopratulto poi dall' ittiolito che vi scoperse il Cav. Francesco Beggiato direttore meritissimo del Museo Civico di Vicenza. In questo esemplare si scorgono abbastanza bene conservati due terzi del corpo, compresa la testa, ed i caratteri presentali dalla forma di questa, dalle mandibule, dalla pinna pettorale e dalle squamme mi fecero in esso riconoscere una nuova specie del genere Pholidophorus che descrissi sotto il nome di Pholidophorus Beggialiemus $\left.{ }^{*}\right)$. Dopo il Fortis non si trova più alcuno che si sia occupato di queste piante, e fu soltanto dopo che io posi in chiara luce, col mezzo dei fossili, come la Calcarea ammonilica delle Alpi Venete spettasse al Giura, che si pote valutare quanto dovesse riuscire importante l'esalta conoscenza

(*) Descrizione di una nuova specie di Folidoforo, con una tavola, negli Alli del R. Istituto Veneto. Vol. XI. Ser. III. 1866. 
delle piante di Rotzo, giacchè la loro giacitura sottoposta alle stratificazioni della Calcarea ammonitica, rendera assai probabile che potessero essere coeve alla tanto celebrata Flora Oolitica della Contea di York.

Fino dal 1830 nel mio Coup d'oeil sur les terrains stratifiés des Alpes rénétiennes, io poneva nella serie oolitica inferiore lo strato a piante fossili di Rotzo, ed annunciava poscia le analogie che mi sembravano esistere tra la Flora di Rotzo e quella di Scarborough in una comunicazione fatta all' I. R. Istituto Geologico di Vienna nella tornata del 4 Maggio 1832 ed in varii altri mici scrilli che videro la luce dipoi. Lo strato con piante fossili che prima si scoperse sul monte Spitz di Rotzo, non solo ricompare nella soltoposta Val d'Assa e lungo la strada che conduce a Mezza Selva, ma estese le mie indagini ad altri punti delle vicine montagne, lo potei riconoscere lungo il confine occidentale della Provincia vicentina nei monti di Marana e Durlo e nella Contrà Lovati nel Comune di Crespadoro. Oltre a questo strato, che è ricoperto dalla calcarea a Terebratula Rotzoana, Scruur., ve ne sono inferiormente degli altri, che alternano con una calcarea argillosa ora grigio-oscura, ora giallo-bruna, zeppa di conchiglie assai mal conservate, e nella parte più bassa di questa serie, non di rado s' incontrano dei piccoli strali di carbone, la cui grossezza in senso verticale non eccede i quattro centimetri. Questa serie accquista maggior potenza procedendo nella provincia di Verona, ore gli strati dell' oolite con piante fossili si ravvisano bene sviluppati in moltissimi luoghi, e principalmente sul Monte Pernigotti presso S. Bartolomeo, sul Monte Raut, alla Giazza, a Bienterle e sul Monte Alba presso Campo Fontana, in Comune di Selva di Progno; a Scandolara e nella Valle delle Ortighe, in Comune di Badia Calarena; ai Coroli di Velo, sul Monte Parpari ed a Garzòn, nel Comune di Velo; nella Valle Zuliani e nella Valle di Salaorno presso Roverè di Velo; nella Valle di Squaranto e nella Valle dell'Anguilla, in Comune di Grezzana. Gli scavi ch' io feci operare in tutti questi luoghi, mi posero in grado di adunare un numero cosi ragguardevole di esemplari da poter proclatmare i depositi delle Alpi Venete, come i più ricchi dopo quelli della Contea di York. Nè si limitano alle sole montagne dei Sette Comuni Vicentini e dei Tredici Comuni del Veronese, chè li veggiamo spuntar fuori sotto 
il Calcare ammonitico, e ricomparire nel Tirolo meridionale sul Monte Malèra, alla bocea di Trappola presso Revolto nel Comune di Ala, ed al nord di Roveredo nelle montagne che fiancheggiano la Valle dell'Adige presso Volano.

In Europa non troviamo altri indizi della Flora oolitica, all' infuori di quelli che ci sono porti dalle Alghe trovate negli scisti varicolori della Toscana dai prof. Meneghini e Savi, e dai banchi di combustibile del Capo Mondego in Portogallo, ove lo Sharpe, seguendo la serie secondaria lungo la costa da Buarcos fino al Capo testè nominato, potè riconoscere come i banchi carboniferi che ivi si scavano, sieno adagiati sopra una potente formazione calcareo-marnosa, in cui si osservano parecchie conchiglie proprie della serie oolitica inferiore, e sieno ricoperti da un'arenaria cui s'intercalano degli strati calcarei, che contengono i fossili caratteristici dell'oolite media. La sola pianta ch' egli potè scorgere nelle marne interposte agli strati di carbone è quella specie di Cicadeacea, cui Morris diede il nome di Zamiles gramineus identificandola al Cycadites gramineus di Phillips, trovalo a Saltwick nel Yorkshire.

I depositi di combustibile del Portogallo segnerebbero al grado $40{ }^{\text {mo }}$ di latitudine sellentrionale, il limite meridionale di una zona che, come vedemmo, si estende fino al $\mathbf{3 8} .^{\mathrm{m} \theta}$ grado, e nella quale sono compresi tutti i depositi di piante fossili che si rinvennero finora in Europa nei varj piani dell' oolite.

Quando nel 1850 il chiarissimo Prof. Unger stampara la sua opera intitolata Genera el Species Plantarum fossilium, le piante realmente proprie dell' oolite giungevano a sole $\mathbf{1 6 0}$ specie. Possiamo ora affermare che le scoperte fatte dopo quell' epoca, fra le quali meritano il primo luogo quelle che si fecero nelle Alpi Venete, abbiano di gran lunga aumentato il numero delle specie di questa Flora.

È perciò che nel presente Volume, il quale contiene le sole Acotiledoni, se ne troveranno descrille ben $\mathbf{1 4 3}$ specie. E questo numero si troverà composto di un Fungo, 59 Alghe, 4 Calamitee, 5 Equisetacee, 94 Felci e 4 Licopodiacee, fra cui $\mathbf{3}$ generi e $\mathbf{5 5}$ specie nuove; cioè due generi e sei specie di Alghe, due specie di Calamitee e due Equisetiti, tre generi 
c ventitrè specie di Felei, cosi riuscendo aumentata di oltre un quarto questa sola parte della Flora dell' oolite.

Nel secondo Volume, che vedrà la luce fra breve, saranno comprese le Monocoliledoni e le Dicotiledoni Ginnosperme, fra le quali le Cicudeacee e te Conifere, che colle loro svariate forme e l'abbondanza dei loro resti formano uno dei caratteri principali della Flora dell' Oolite; ed in fine dell'Opera si troveranno esposte le conclusioni generali a cui mi potè condurre lo studio dei resti vegetali trovati in questa formazione, accompagnate da alcuni prospetli destinati ad indicarne la distribuzione geografica e geologica e le affinita che palesa questa Flora, paragonata con quelle dei terreni a lei più prossimi nell'ordine cronologico delle formazioni.

Possiamo però fin d'ora osservare come, specialmente nella serie oolilica inferiore, sieno dominanti i resli delle Calamarie, delle Felei, delle Cicadeace, delle Conifere, e questo fatto, che si nota pur anco nelle Flore del Keuper e del Lias e si ripete nell'Argilla di Weald induce a ritenere, che durante l'epoche nelle quali si deposero tulti questi terreni, le condizioni necessarie alla regetazione di queste piante si sieno mantente pressochè eguali in ciascheduno dei detti periodi.

Ma sebbene la comune prevalenza di queste classi in confronto delle altre, componenti le Flore di queste diverse formazioni, stabilisca una ben marcata analogia fra di loro, pure l'aumento considererole nel numero delle Felei, delle Cicadeacee e delle Conifere che si osserva nell'oolite, ci trae a ragionevolmente supporre, come segnando quest'epoca il punto culminante della loro regetazione, le condizioni del globo fosscro in allora, pii che negli altri periodi, favorevoli al loro svolgimento.

Il celebre Brongniart da più anni arrertiva alle affinità sussistenti fra queste Flore, notando tuttavia come fossero rare nell'oolite le Felci a nervalure relicolate, si frequenti nel Lias; come vi dominassero le Otozamiti e le Zamiti, ossia le Cicadeacee che più si approssimano alle viventi, e diminuisse il numero delle Nilssonie e dei Pterofilli che più si discostano da quelle della Flora attuale, e come alla fin fine le Conifere vi fossero più numerose di quello si mostrino nel Lias. Queste conclusioni dettate da quel raro talento che sa riunire la precisione nei particolari all'ampiezza 
delle vedute generali, e che traluce in ogni scritlo di quell illustre naturalista, furono in gran parte confermate anche dalle scoperte posteriori, e solo osserviamo come tra le piante rinvenute nelle Alpi Venete, appariscano alcune forme che segnano una più precisa separazione fra la Flora dell'0olite e quelle delle formazioni vicine. Pure questa Flora ci presenta un carattere comune a quelle nella prevalenza delle piante terrestri, sicuro indizio di suolo emerso durante quell' epoca in più punti del globo. E in quali condizioni fossero poste queste terre, sorte dal seno del vasto mare che in allora copriva tanta parte della superficie terrestre, ci viene svelato dalla natura stessa della loro vegetazione, paragonata a quella parte della Flora presente, che più vi si avvicina.

Imperciocchè se prendiamo ad esaminare le stazioni delle piante che attualmente appartengono a quelle classi e famiglie, che vediamo dominare nella Flora dell'oolite, noi redremo le Equisetace, frequenti nelle regioni temperate, mancare nell' emisfero australe, menomarsi fra i tropici, ma quivi approssimarsi per la maggior grandezza a quelle dell' oolite; le Felei verdeggiare numerose nelle regioni tropicali bagnate dal mare, ridursi a poche nella Flora continentale dei paesi ealdi e dei temperali quando privi d'acqua o di sufficiente umidità, diminuire ancor più dal Cancro al Polo, aumentarsi ed occupare gran parte del suolo nelle isole poste al di là del Capricorno.

Le Cicadeacee, scarse nelle regioni dell' antico mondo collocate fra i tropici, svolgono rigogliose le pinnate lor frondi in quelle poste vicino all'Equatore nel nuovo; aumentano di numero al di là del Capricorno e s'intrecciano alle Conifere lungo le coste e nelle terre australi della Nuova Olanda e del Capo di Buona Speranza.

Le Conifere rappresentate nell'oolite da forme che si avvicinano a quelle ora viventi nelle terre australi, sebbene in più scarso numero, palesano tultavia anch' esse una vegetazione che rammenta quella delle regioni più temperate del globo e più vicine al mare. Pure il carattere più essenziale e rilevalo della Flora oolitica è specialmente fornito dal gran numero di Cicadeacee che in essa dominava: caraltere che stabilisce la sua analogia colla Flora che ora si osserva in alcune regioni dell'emisfero australe, men- 
tre all' invece reggiamo, che durante il periodo oolitico, le piante di questa classe lasciarono frequenti traccie della loro presenza in piì luoghi dell'Europa, entro una zona che, come vedemmo, si estende dal 40. ${ }^{\mathrm{mo}}$ al $38{ }^{\text {mo }}$ grado di latitudine settentrionale.

Da questi fatti e da queste analogie noi saremmo indotti a conchiudere, come le terre emerse di questa parte del globo, fossero in quell' epoca circondate da un vasto oceano, e godessero all' incirca delle medesime condizioni di temperie e di suolo che favoriscono nell' epoca presente la vegetazione di quelle famiglie, cui appartengono le forme vegetali finora scoperte nei varj piani della formazione oolitica. Ma su queste considerazioni generali torneremo con maggior fondamento in fine di quest'opera, dopo aver compiuta la descrizione di tutti i resti vegetali rinvenuti nei terreni dell'epoca oolitica, ed avere approfittato di tutti quei lumi che da ulteriori scoperte potessero in questo frattempo esserci somministrati. 


\section{FLORA FOSSILIS FORIITIONIS OOLITHICAE.}

\section{ACOTYLEDONES.}

\section{Classis I. FUNGI.}

I

Funghi hanno un' assai limitata importanza nella storia delle spente vegetazioni, e ristretto è pure il numero delle specie fossili fin quì osservate.

Nel terreno carbonifero della Valle di Llangollen, nella Contea di Denbigh in Inghilterra, il sig. Borvman trovava due esemplari di una impronta, le cui analogie co Polipori viventi indussero gli autori della Flora fossile della Gran-Breltagna ad apporvi il nome di Polyporites Bowmani (Foss. Flor. of Great Brit. Vol. I. tab. 65). Anche il Garpolithes umbonatus dello Sternberg (Vers. Flor. Vorw. Tom. I. tab. 9. fig. 2.), rinvenuto nella formazione del Litantrace della Boemia, deve, secondo i signori Lindley, Hutton, Brongniart. ed Unger, riferirsi alla Classe dei Funghi; ed egualmente unªltra impronta che, giusta il Brongniart, vi assomiglia di molto, e proviene dal terreno carbonifero della Sardegna.

Meglio caratterizzate di queste sono le poche specie di Funghi epifilli recentemente descritte e figurate dal Geinilz (Preisschrift 1855. - Steinkohleuformation in Saxen, 1856), e quella scoperta dal Göppert sulle foglie dell'Hymenophyllites Zobelii (Göpp.) nel terreno carbonifero della Slesia, e cle illustrò sotto il nome di Excipulites Neesii (Göpr. System. Filic. Foss. pag. 262, tab. 36. fig. 4).

Queste forme dell ${ }^{\circ}$ epoca carbonifera segnano la prima apparizione di specie spettanti alla Classe dei Miceti. Dopo di che non ne veggiamo piu traccia fino al periodo in cui si deposero i varj piani del sistema giura-liassico, ove quattro specie furono osservate nel Lias, e due nell' Oolite. Mancano nuovamente nell'argilla di Weald, ed in tutto il sistema cretaceo; e tornano a farsi vedere soltanto nei terreni terziarj, ove si mostrano più numerose, giungendo ivi a tredici le specie conosciute.

Le due specie proprie dell' Oolite sono: il Xylomites irregularis di Göppert, rinvenuto nella Slesia superiore in una formazione chiarita per altri fossili contemporanea ai depositi oolitici con resti vegetali della Contea di York nell' Inghilterra; e quella forma singolare che lo Sternberg descrisse e figurò solto il nome di Algacites intertextus, ma che per le osservazioni del Trevisan deve prender posto tra i Funghi, presentando la mag. gior parte dei caratteri che distinguono il genere Rhizomorpha. 


\section{Ordo I. Hyphomycetes Fries}

\section{Geu. I. Itizonorpintes (Göpp.?) Trevis.}

Filamenta elongata radiciformia, compressa, parallela, ramosissima, ramulis linearibus irregulariter reticulato-anastomosantibus.

TREVIs, 1849 in miss. edit. II. Nomencl. Algarum.

? Rhizomorphites Göpr. in Brown Gesch. der. Nat. pag. כ. - Brons Ind. Palaeont. P. I. pag. 108 .

\section{Hhizomorphites intertextus Trevis.}

R. filamentis ramosissimis, longitudinaliter striatis; primariis latioribus subparallelis; ramulis linearibus, planis, intertexlis, irregulariter reticulato-anastomosantibus.

Rhizomorphites intertextus 'Trevis. 1849 in mss. edit. II. Nomencl. Algarum.

Algacites intertextus Sтввкв. Vers. Flor. Vorw. II. pag. 37. tab. 21. fig. 6. - Urg. Gen. et Spec. plant. foss. pag. 31. - Brons Ind. Palaeont. P. I. pag. 25. - Giebre Deutschl. Petref. pag. $15, \mathrm{n}^{\circ} 137$.

In schisto jurassico ad Solenhofen? - Ectypum hujus singularis speciei in Musaeo Universitatis Friburgensis asservatur (Stemb. loc. cit.)

Nella figura pubblicata dallo Sternberg si scorgono parecchi rami del diametro $d^{2} u n$ millimetro e mezzo, percorsi da strie longitudinali continue, dai quali si propagano in forma pimnata innumerevoli ramoscelli piani, lisci, patenti, che unitamente ai primi si anastomizzano fra di loro ad angolo più o meno retto, e formano una specie di rete a grandi maglie irregolari.

Il Cav. Vittore Trevisan in un lavoro tuttora inedito, che deve far parte della seconda edizione del suo Nomenclator Algarum, esclude dalle Alghe, per collocarlo tra i Funghi, l'Algacites intertextus dello Sternberg, scorgendo in esso molta analogia col vivente genere Rhizomorpha, e specialmente colla $R$. subcorticalis (Pers.). In quanto al genere Rhizomorphites (Göpp.), citato nella prima Parte dell'PIndex Palaeontologicus di Bronn (pag. 1085), esso si riferisce a quella Rhizomorpha cle il Göppert scoperse nelle ligniti terziarie della Slesia (Göpr. System. Filic. foss. pag. xxr). Non è però a mia cognizione che quest'ultimo naturalista abbia pubblicata alcuna descrizione del genere Rhizomorphites. 


\section{Ordo II. Gasteronycetes Fries \\ Gen. H. XYLourtes Ung.}

Perithecium vel receptaculum epiphyllum, crassum, durum, medio umbonatum.

UNG. Ghlor. protog. I. pag. 5 ; Syn. plant. foss. pag. 19 ; Gen. et Spec. plant. foss. pag. 58. - Göpr. Gatt. foss. Pfl. pag. 109 ; Foss. Flor. der. mitl. Jur. pag. 142. - Massat. Piant. foss. terr. terz. Vicent. pag. 57.

\section{Xylomites irregularis Göpp.}

X. perithecio rotundato vel oblongo, disco centrali integro impresso subconcavo.

Xylomites irregularis Göpr. Foss. Flor. der mitl. Jur. pag. 142, tab. I. fig. 12-17. Bronn Ind. Palaeont. P. I. pag. 1376. - UNG. Gen. el Spec. plant. foss. pag. 38. - Gieber Deutschl. Petref. pag. 18, n. ${ }^{\circ} 166$.

In argilla calcarifera ferruminosa (Thoneisenstein) ad Wilmsdorf Silesiae.

Questo Miceto epifillo è stato rimarcato dal Göppert in un’argilla ferruginosa, spettante al gruppo oolitico inferiore, sopra alcuni frammenti di foglie lineari allungate ed acuminate, le quali per le nervature parallele e lievemente convergenti verso l'estremità, da cui sono longitudinalmente percorse, sembrano avere appartenuto a qualche specie di Cicadeacea.

Esso si manifesta solto l'aspetto di minutissimi punti brunicci di varia grandezza, sparsi sulle foglie negli spazj che stanno framezzo alle nervature. Sottoposti al microscopio, si mostrano rotondato-allungati, ed alcun poco più elevati nella parte mediana, ove parecchi nel punto centrale e più sporgente palesano una lieve depressione circolare. (Vedi Görp. loc. cit. fig. 17.)

\section{Classis II. ALGAE.}

Le impronte riferibili a specie di questa Classe cominciano a farsi vedere nelle formazioni fossilifere più antiche. I terreni delti una volta di transizione e della grauwacke, ora chiamati Siluriani e Desoniani, la cui Flora fu di recente illustrata dalla maestra penna del Göppert, ci hamno svelato una Conferva, e non poche Fucoidee e Floridee che prima non erano note, e di cui il numero maggiore, scoperto nella formazione Siluriana d’Ame- 


\section{$-4-$}

rica, trovasi descritto e figurato nel Volume I. della Paleontologia dello Stato di Nuova York di Giacomo Hall. Scemano d'assai negli strati deposti nelle epoche immediatamente successive, e mancano finora del tutlo nell’arenaria variegata.

Nel Muschelkalk il solo Sphaerococcites Blandowskiamus di Goeppert, nel Keuper due Confervacee ed una Fucoidea, nel Lias due Fucoidee e sei Floridee, ne segnano la ricomparsa, che si fa maggiormente palese nella serie oolitica mercè la presenza di due Confervacee, di quattro Fucoidee e di trentadue Floridee.

Tornano a farsi rare nell'argilla di Weald, nella quale l'Ettingshausen ci ha non ha guari fatto conoscere, oltre al Confervites fissus del Dunker, tre nuove specie, cioè: il Confervites setaceus, il Sargassiles Partschii, e lo Sphaerococcites chondriaeformis. Finalmente si mostrano in gran copia nei varj piani della formazione cretacea e dei terreni terziarj.

Limitandoci alle Alghe trovate finora nella serie oolitica, osserveremo come si veggano precipuamente abbondare nelle calcarie scissili di Solenhofen nella Contea di Pappenheim, riferite dall' Orbigny alla parte superiore del suo piano Oxfordiano, ma poste dai piu dei Geologhi con buone ragioni nel Coral rag. Cinque sole specie ci offrono i terreni compresi nel gruppo dell' Oolite inferiore, tre delle quali proprie dell’ Inghilterra, e due da me rinvenute nelle Alpi Venete. Sembra pure che alla parte inferiore di questo gruppo si debbano riportare le sei specie scoperte negli scisti varicolori della Toscana dai Professori Meneghini e Savi, alcune delle quali sono riferite a forme che si credevano finora proprie soltanto di terreni più recenti. Altre due ne trovò $1^{\circ}$ Unger negli strati ggimassici dell’Austria inferiore, ed una il Kurr nel Giura superiore di Gerhausen presso Ulma.

Da questo novero sono escluse tutte quelle piante, le quali, sebbene abbiano sempre figurato tra le Alghe negli elenchi delle specie oolitiche, devono ora prender posto tra le Conifere, specialmente dopo le recenti osservazioni del Brongniart (1) e dell' Unger (2). Sono queste: il Baliostichus ornatus dello Sternberg, e tutte le specie del genere Caulerpites, che spettano a questa formazione, ad eccezione del Caulerpites tortuosus di Presl, che, seguendo Brongniart ed Unger, ritenni fra le Algुhe, proponendolo però qual tipo di un nuovo genere.

Tra le Alghe, di cui porgo la descrizione nel presente lavoro, si vedrà ommesso il Cystoseirites mutuns dello Sternberg, specie che il Bronn nell’ultima edizione della Lethaea geognostica colloca fra quelle dell' Oolite. Tanto questo naturalista, quanto il Giebel (Deutschland. Petref. pag. 7), citano a proposito di questa specie la fig. 1. tav. 7. del Volume II. della Flora der Vorwelt; ma questa figura corrisponde invece allo Sphaerococcites affinis (Sternb.), a cui per errore venne dallo Sternberg medesimo riferita la fig. 1. tav. 6. dello stesso Volume; figura che spetta invece all’Halymenites Schnitzleinii (Sternb.). Inoltre il

(1) Tableau des genres des végétaux fossiles. (Extr. du Dict. Univers. d' Hist. Nat. pag. 8 et 69.)

(2) Ueber einige fossile Planzen aus dem Zithographischen Schiefer von Solenhofen, mit. 2. Taf. 


\section{- こ -}

Brom aggiunge alla descrizione una figura (Lethaea geognostica, tav. 14. fig. 8.) affatto eguale alla fig. 1. tav. 18. Vol. II. della Flora der Vorwelt, che rappresenta il Cystoseirites taxiformis (Sternb.), specie clıe il Göppert e l'Eutingshausen hanno identificata col1'Araucarites Sternbergii di Göppert.

Nella Flora der Vorwelt non esiste delineato il Cystoseirites mutans, sebbene vi sia descritto e indicato provenire dai calcari scissili di Solenhofen. Però nelle correzioni stampate di poi dall' autore è dichiarato come $1^{1}$ impronta, su cui aveva creduto fondare questa specie, non appartenga al regno vegetale.

Altri errori sono corsi in quest' opera, $d^{\prime}$ altronde pregevolissima, i quali furono poscia raddrizzati dallo Sternberg stesso in un foglio pubblicato qualche tempo dopo; ma questo foglio non fu abbastanza diffuso, imperciocchè vegggiamo ripetuli gli errori medesini da quasi tutli gli autori, nelle cui opere è citata quella dello Sternberg.

Parlando delle Alghe trovate nell' Oolite, non devesi ommettere di rammentare come i caratteri generici e specifici di queste dilicate pianticelle riescano troppo fuggevoli ed incerti neggli esemplari fossili, perchè se ne possa stabilire con sicurezza la distinzione, e come perciò debbansi considerare quali indicazioni provvisorie delle forme che ci presentano. Nè dobbiamo riposare sulla presenza di taluna tra queste forme per determinare l'età del terreno in cui si trova rappresa, giacchè è ben noto agli Algologhi quali differenze cagioni l'età nella stessa specie, e come di spesso le singole parti di un medesimo individuo, prese separatamente, presentino forme le più svariate. Dal che ne consegue, che i frammenti per lo più imperfelti ed alterati dalla fossilizzazione delle piante appartenenti a questa Classe tornino assai poco gioveroli pel riconoscimento delle formazioni, qualora non sieno accompagnati da altri fossili. Nè tampoco possiamo far calcolo di quegli esemplari che appariscono meglio conservati, e di cui più costanti ci si palesano i caratteri. Impercioccliè se è vero che nella distribuzione dei resti organici quanto piu alta è l’organizzazione, tanto piu ricisamente sia definito il suo orizzonte stratigrafico, si può quindi ragionevolmente ammettere che segetali di una Classe tunto inferiore, quali sono le Alghe, e tanto acconci a sopportare $i$ cambiamenti fisici, possano aver contimuato ad esistere passando incolumi per que' grandi mutamenti a cui non poteva resistere la vita animale. Questo principio, esternato dall’ illustre Murchison nel far conoscere il niun valore caralteristico di alcune Fucoidi trovate promiscue ai terreni cretacei e terziarj d'Italia (1), viene ora possentemente avvalorato dall'avere il Prof. Meneghini rinvenuto alcune di queste stesse Fucoidi nei calcari $e$ scisti varicolori della Toscana, che altri fossili manifestano appartenere decisamente al Giura; con che è tolta a questa specie ogni importanza per la classificazione delle roccie in cui si ritrovano.

(1) R. I. Murchison, On the structure of the Alps, Apennines and Carpathians, etc. (Quart. Journ. Geolog. Soc. Lond. 1848, pag. 227); e nella traduzione italiana dei Professori Mleneghini e Savi, stampata in Firenze nel 18b̆1, alla pag. 117. 
Però, sebbene le Alghe non debbano per queste considerazioni valutarsi opportune alla sicura identificazione delle formazioni, tuttavia la loro presenza potrà essere utilmente adoperata ad investigare la natura della vegetazione che popolava le acque nei diversi periodi geologici.

In quanto poi all’ordinamento delle specie descritte nella presente Flora stimai fosse cosa intempestiva applicare ad esse le classificazioni non ha guari introdotte rispetto alle Alghe viventi, e basate sulle recenti scoperte intorno aggli organi della riproduzione, essendo lo studio delle Alghe fossili ancora troppo poco avanzato per autorizzarci ad instituire minuli confronti colle specie attuali. Credetti perciò miglior consiglio attenermi alla classificazione adoltata dal chiarissimo Professore Unger nell'opera intitolata Genera et Species plantarum fossilium, unico lavoro generale che si possegga sulle piante fossili.

\section{Ordo III. Confervaceae Endlich.}

Geu. IH. Covenvites Brong.

Frons filiformis; fila libera, simplicia vel ramosa, articulata vel continua, entosperma.

Broxa. Prodr. pag. 13; Hist. végét. foss. I. pag. 55 ; Tabl. gen. végét. foss. pag. 7. Sternib. Vers. Flor, Vorw. II. pag. 19. - Lidircir. Gen. plant. Suppl. III. pag. 55. - UxG. Gonspect. Flor. primord, in Ghlor. Protog. pag. xxvr.; Gen. et Spec. plant. foss. pag. 7.

\section{Confervites Veronensis Zign.}

Tar. I. fig. 1. 2.

G. filamentis simplicibus, rectis rel subincurris, fasciculatis rel effusis, rigidiusculis, septis inconspicuis.

In calcareo scissili formationis oolithicae inferioris ad M. Pernigotti prope San Bortolamio in Valle Tanara, Provinciae Veronensis.

La presente specie consta $d^{2}$ un aggregato di filamenti semplici, capillari, aventi un colore di rugine più o meno carico, ora ritti e rimiti a fascelto, ora curvati in varie direzioni, confusamente sparpagliati, e fra loro in più guise incrocicchiati.

La figura 1.a porge il primo di questi due aspetti, e nella figura $2{ }^{a}{ }^{a}$ è delinealo $l^{2}$ altro. $L^{\prime}$ esemplare raffigurato in quest'ultima, osservato ad occhio nudo, sembra in alcuni punti ramificarsi; ma soltoposto ad un conveniente ingrandimento, lascia scorgere di leg- 


\section{$-7-$}

gieri come questa apparenza sia dovuta alla separazione di filamenti per alcun tratto prima riuniti per semplice sovrapposizione.

Quest'Alga, ch'̀̀ il primo esempio di una Conferva trovata nell' Oolite, si assomiglia ad alcune specie rinvenute in altre formazioni. Quelle a cui maggiormente si avvicina sono il Confervites fasciculatus (Brong. Hist. végét. foss. I. pag. 35. tab. I. fig. 1.) degli strati crelacei dell' isola di Bornholm, ed il Confervites setaceus (Etringsuausen, Beitrag zur näheren Kenntniss der Flora der Wealdenperiode, pag. 9. tab. III. tig. 3.) del terreno Wealdiano delle vicinanze di Krems nell ${ }^{2}$ Austria inferiore. La nostra specie si distingue però dalla prima per avere $\mathbf{i}$ filamenti affatto privi di alcun indizio di setli; dalla seconda per essere assai più tenui e spesso incrociati, nè cotanto rigidi da frangersi, come è indicato nella descrizione e figura del Confervites selaceus dell ${ }^{2}$ Ettingshausen. Mostrano piutlosto di avere posseduto un grado di flessibilità sufficiente a potersi leggermente curvare senza rompersi.

\section{Gen. Hv. Evcoxochadur Zign.}

Frons fistulosa?, ramosa, obtusa, caule, ramis ramulisque huc illue irregulariter intumescentibus, apice clavato-incrassatis.

\section{Sincoelocladium tortuosum Zign.}

E. fronde pinnatim ramosa, ramis dichotome-ramosis cauleque irregulariler submoniliformi-tumidis et constrictis, ramis ramulisque apice clavato-incrassalis.

Caulerpites torluosus Prest in Stenxi. Vers. Flor. Vorw. II. pag. 105. Lab. 29. fig. 1. Endich. Gen. plant. Suppl. III. pag. 54. - UNG. Gonspect. Flor. primord. in Ghlor. Prolog. pag. xxv.; Gen. et Spec. plant. foss. pag. 7. - Brosx Ind. Palaeont. pag. 2ö1. - Gieber Deutschl. Petref. pag. ̋̈.

Codites? torluosus Brong. Tabl. gen. végél. foss. pag. 10 .

In calcareo scissili formationis oolithicae mediae ad Solenhofen.

L' esemplare figurato dallo Sternberg ha la fronda alquanto allargata alla base. Poi rapidamente si ristringe, si ramifica in forma pimnata, e palesa in ogni sua parte dei rigonfiamenti irregolari che lo fanno sembrare bernoccoluto. I rami si sparpagliano tortuosamente, si dicotomizzano, ed offrono le estremità ingrossate in forma di clava.

Presl ed Unger pongono questa specie nel genere Caulerpites, col quale non avrebbe di comune che il solo carattere della fronda ramosa ed ottusa. Questi due autori però 
siustificano tale determinazione attribuendo la mancanza degli altri caratteri allo stato imperfetto dell' esemplare. Tuttavia è un fatto che l'Alga figurata nell' opera dello Sternberge non presenta la fronda laciniato-pinnata o fogliaceo-squammosa del genere Caulerpites, in cui fi: posta da Presl; e manca della fitta pelurie e dei granellini che distinguono il genere Codites, a cui la riferisce, benchè con qualche dubbio, il Brongniart. Perciò mi è sembrato dovernela separare, proponendola a tipo di un nuovo genere, caratterizzato specialmente dal modo di ramificazione e dalla irregolare intumescenza della fronda.

\section{Ordo IV. Phycene Endlich. \\ Gen. V. Copres Sternb.}

Frons spongiaeformis, cylindrico-lubulosa, inordinate aggregata, pilis creberimis vestita, granulis coloratis dense obsita.

Sterre. Vers. Flor. Vorw. II. pag. 20. - Eirdich. Gen. plant. Suppl. III. pag. 54. Uxe. Conspect. Flor. primord. in Chlor. Protog. pag. xxvr.; Gen. et Spec. plant. foss. pag. 9. - Brokg. Tabl. Gen, végét. foss, pag. 9.

\section{Codites serpentinus Sternb.}

C. frondibus caespitose aggregatis, flagelliformibus, diffusis, teretibus, hine inde incrassatis constrictisque, apice clavatis, saepissime simplicibus, raro dichotomis.

Codites serpentinus Stennd, Vers. Flor. Vorw. II, pag. 20, tab, 3. fig. 1. - Endicr. Gen. plant. Suppl. III. pag. 5ั4. - Usg. Conspect. Flor. primord. in Chlor. Protog. pag. xxvr.; Gen. et Spec. plant. foss. pag. 9. - Brosi Ind. Palaeont. P. I. pag. 518 . Leth. geognost., edit. 3. fase. 2. pag, 40. tab. 14. fig. 11. - Gieber Deutschl. Petref. pag. 6, n. 52.

Codites difformis Brosc, Tabl, gen. végét, foss. pag. 10 ä (pro parte).

In calcareo lithographico ad Pappenheim.

$L^{\prime}$ aspelto, solto cui si presenta quest' $\mathbf{A l g a}$, si è quello $\mathrm{d}^{\prime}$ un aggregato di frondi affastellate nella parte inferiore, le quali poi si estendono indistintamente per ogni verso, isolandosi e prolungandosi però di più nella parte superiore, Si mostrano tubulari, irregolarmente rigonfie, di tratlo in tratto ristrette o strozzate, per lo più semplici, assai di rado dicotome, e colle estremità ora tronche, ora rotondate e claviformi. Ove è maggiore l'intumescenza il diametro della fronda giunge fino ai quindici millimetri. 
La figura data dallo Sternberg rappresenta questa specie nella sua naturale grandezza; quella del Bronn è una semplice riduzione a dimensioni minori della stessa figura.

\section{Codites erassipes Sternb.}

C. frondibus aggregatis?, inaequalibus, crassis, basi tuberose incrassatis, longiore medio et apice incrassato oblusissimo, breviore cylindraceo subfalcato obtuso.

Codites crassipes Sterne. Vers. Flor. Vorw. II. pag. 20. tab. 2. fig. 5. - Endich. Gen. plant. Suppl. III. pag. 5ै4. - UNG. Conspect. Flor. primord. in Chlor. Prolog. pag. xxrr. Gen. el Spec. plant. foss. pag. 9. - Bronn Ind. Palaeont. P. I. pag. 518. - Gieber Deutschl. Petref. pag. 6. n. ${ }^{\circ} 33$.

Codites difformis Brong. Tabl. gen. végét. foss. pag. 9 (pro parle).

Eodem in loco cum priore.

Da una base rigonfia, larga quattro centimetri ed alta tre e mezzo, $\mathrm{s}^{2}$ inmalza verticale un ramo tubulare della fronda, lungo sedici centimetri e tre millimetri, dapprima ristretto, che poi gradatamente s'ingrossa, finchè giunge nel suo mezzo ad un diametro di ventiquattro millimetri; poi torna a ristringersi fino ai selte millimetri e scorre per quattro centrimetri, curvandosi alcun poco e lievemente allargandosi fino alla base dell’ intumescenza ovoidea, che ne forma l'estremità. Questa è lunga due centimetri e mezzo, e larga due.

Lateralmente alla base si distende obbliquamente un altro ramo lungo sei centimetri e selte millimetri, e largo circa due centimetri, che si curva alquanto all' insu, diminuisce gradatamente di diametro progredendo verso l'estremità, non palesa alcuin rigonfiamento, e presenta $l^{p}$ aspetto di una falce rivolta col taglio verso la parte superiore.

Due frammenti staccati, che si osservano presso la base nell' esemplare figurato dallo Sternberg, fanno supporre che altre frondi potessero esservi aggregate.

In questa specie i peli caratteristici del genere sono meno visibili di quello lo sieno nell' antecedente.

Dubita lo Sternberg clie questa specie sia una semplice varielà del Coclites serpentimus. Il Brongniart opina invece che l’una e l'altra sieno forme accidentalmente diverse della stessa specie, e nell' elenco delle piante dell' Oolite, posto in fine della sua preziosa operetta intitolata Tableau des genres des végétaux fossiles, le riunisce sotto il nome di Codites difformis, col quale non si deve però confondere il suo Fucoides difformis, descritto e figurato nell'Histoire des végétaux fossiles, e che ora è il Chondrites difformis dello Sternberg, specie ben diversa e trovata nel terreno cretaceo inferiore di Bidache presso Bajona. 


\section{$-10$}

Le notabili differenze che presentano le due specie di Codites testè descritte, mi hanno indotto a seguire l'esempio dato dall'Unger nel suo Genera et Species plantarum fossilium, ed a tenerle per ora separate, ben lontano però dal voler con ciò contraddire in via assoluta all'opinione di una così illustre autorità, quale si è quella del Brongniart.

\section{Codites Grantzianus Zign.}

Tav. I. fig. 3.

C. fronde solitaria crassa erecta, dein incurva apice obtusa, basi ramulo uno brevissimo aucla, sursum ramis tribus subaequalibus elongatis incurvis angulo acuto egredientibus ornata, granulis oblongis elevatis confluentibus conspersa.

In calcareo lithographico formationis jurassicae mediae ad Solenhofen.

La fronda di questa specie, quantunque nell' esemplare sia alquanto compressa, lascia tuthavia vedere la consistenza propria del genere Codites. Essa scorre ritta per un tratto di oltre a sei centimetri, poscia si va incurvando fino all’apice, che è ottuso. Alla sua base si osserva lateralmente il rudimento di un ramo brevissimo, e nel punto ove essa comincia a curvarsi ne sorgono ad angolo acuto altri tre molto più allungati, che, discostandosi alcun poco, prendono la stessa curva della parte superiore della fronda, piegandosi per lo stesso verso, e terminando com'essa.

Due di questi rami sono inseriti un po più abbasso del terzo, ed havvi sì poca differenza tra le loro dimensioni e quelle della fronda, che quest'ultima si direbbe a prima gimta divisa nella metà superiore in quattro rami, e quasi digitato-flabellata.

Il diametro tanto della fronda che dei rami sta fra $\mathrm{i}$ tre e $\mathrm{i}$ quattro millimetri, e la lunghezza, senza calcolare la curva, raggiunge i quatlordici centimetri e mezzo.

Questo esemplare, proveniente dai calcari scissili di Solenhofen, mi fu inviato dal Dott. Augusto Krantz di Berlino, a cui l'ho dedicato. Esso non corrisponde ad alcuna delle specie fossili conosciute, ed il solo genere in cui possa per ora prender posto si è il genere Codites.

\section{Geu. V. Evcoecites Sternb.}

Frons tubulosa vel vescicaeformis, punctata. Sporangia subulata.

Sterne. Vers. Flor. Vorw. II. pag. 33. - End. Gen. plant. Suppl. III. pag. 54. - UnG. Conspect. Flor. primord. in Chlor. Protog. pag. xxvi.; Gen. et Spec. plant. foss. pag. 9. 


\section{Cneoelites Mertensif Sternb.}

E. fronde oblonga irregulari, sinuosa, nigro-punctatissima, margine sinistro cute cerino-brunnea obtecta.

Eneoelites Mertensii Sterke. Vers. Flor. Vorw. II. pag. 53. tab. 3. fig. 2. - Exdr. Gen. plant. Suppl. III. pag. 54. - Ung. Conspect. Flor. primord. in Chlor. Protog. pag. xxvr.; Gen. et Spec. plant. foss. pag. 10. - Bronw Ind. Palaeont. P. I. pag. 459.; Leth. geogn. edit. 3. fase. 2. pag. 44. tab. 14. fig. 6. - Greber Deutschl. Petrefact. pag. 6, n. ${ }^{\circ} 5$ 4.

In calcareo lithographico ad Pappenheim.

$L^{9}$ unico esemplare esistente si presenta di forma vescicolare rotondato-oblunga, sinuosa, con lieve ristringimento nella parte mediana, e con una espansione laterale nella parte superiore, che termina dividendosi in tre lobi ineguali di forma diversa, di cui il centrale è lineare, lungo sette millimetri, tronco all' estremità, e potrebbe essere il rudimento di un ramo; il superiore è lanceolato, e l'inferiore rotondato, coll' apice sinuato. La superficie, che nel margine si palesa giallo-bruna, è tulta coperta di minutissimi punti neri.

Secondo il Brongniart, i caralteri di questo genere non sarebbero sufficienti a separarlo dal genere Codites. E per vero, potrebbe essere lo stato giovane di una delle due precedenti specie. Per la natura della fronda, e per li punti di cui è cospersa, si avvicinerebbe anche al genere Halymenites, egualmente come per la forma si assomiglia all'Algacites erucaeformis dello Sternberg.

\section{Ordo V. Florideae Lamour. \\ Gen. HII. MÜusteria Sternb.}

Frons coriacea, cylindrica, fistulosa, simplex caespitose-aggregata vel dichotoma, transversim elevato-striata, striis creberrimis interruptis. Sporangia punctiformia, in spatiis inter strias interruptis creberrima, sparsa, frondis substantiae immersa.

StвRкв. Vers. Flor. Vorw. II. pag. 31. (non Deslongch.) - Endr. Gen. plant. Suppl. III.

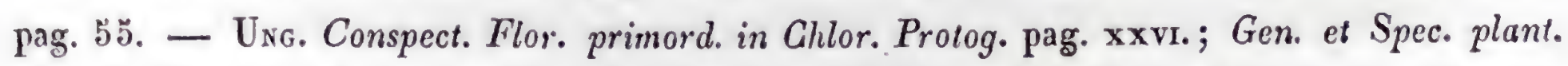
foss. pag. 14. - Brong. Tabl. gen. végét. foss. pag. 11. - Brons Leth. Geogn. edit. 3. fasc. 2. pag. 44.

Fucoides, §IV. Encoelites Broxg. Prodr. pag. 19.; Hist. végét. foss. I. pag. 55. 


\section{Miünsteria encoelioides Bronn}

M. frondibus caespitose-aggregatis, erectis, simplicibus, clavatis, obtusis, in basim ascendentem altenuatis, striis transversis tenuissimis.

Nünsteria encoelioides Brons Leth. Geogn. edil. 5. fasc. 2. pag. 44. tab. 14. fig. 1.

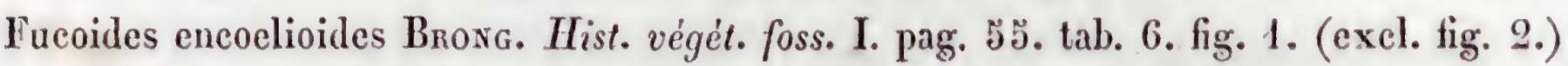

Nünsteria clavata Sterne. Vers. Flor. Vorw. II. pag. 31. - Exde. Gen. plant. Suppl. III. pag. 55. - UrG. Conspect. Flor. primord. in Chlor. Protog. pag. xxvi.; Gen. et Spec. plant. foss. pag. 14. - Bronv Ind. Palaeont. P. I. pag. 746. - BRong. Tabl. gen. végét. foss. pag. 103̈. - Greber Deutschl. Petrefact. pag. 8, n. $^{\circ} 57$.

In calcareo scissili ad Solenhofen.

L'esemplare figurato da Brongniart e da Bronn si scorge composto da cinque frondi riunite per semplice apposizione delle loro estremità inferiori, a cui lateralmente due altre cortissime si aggiungono, che per la loro sottigliezza sembrano doversi considerare abortive. Le cinque maggiori si dipartono con lieve curva dal punto di unione, e gradatamente ingrossando si raddrizzano e prolungano dagli otto ai dieci centimetri. Il diametro di ciascheduna neł punto della maggiore larghezza misura dai sette ai nove millimetri, e tutte poi terminano coll ${ }^{p}$ estremità ottusamente ovata.

Le finissime strie assai avvicinate, da cui è trasversalmente segnata, sono meglio espresse nella figura data dal Brongniart.

Il Brongniart nella sua Histoire des végétaux fossiles (loc. cit.) pose quest' Alga fra gli Encoelites col nome di Fucoides encoelioides; e notò le analogie che offre coll' Encoelium bullosum di Agardh, che vive lango le coste della Francia; coll' Ulva rugosa di Decandolle, ch' è l'Encoelium echinatum di Agardh (Spec. Alg. tom. I. pag. 145); e col Gastridium ovale di Lyngbye, cl’’è la Valonia oxalis di Agardh (Spec. Aly. tom. II. pag. 431).

Stemberg creando il genere Münsteria ve la annoverò, dandole il nome di Münsteria clacata; nome che assai bene risponde alla sua forma, e clie fu poscia ammesso dall ${ }^{2}$ Unger, dal Bronn, dallo stesso Brongniart nel suo Tableau des genres des végétaux fossiles, e da Giebel. Però il Bronn, nella terza edizione della Lethaea geognostica, cangiollo in quello di Münsteria encoeloides, così serbando memoria della denominazione data a questa specie dal Brongniart, che fu il primo ad illustrarla. Ciò essendo consono alle buone regole della nomenclatura botanica, è d'uopo quindi ritenere il nome che le fu imposto dal Bronn. 


\section{Münsteria rermicularis Sternb.}

M. fronde solitaria erecta, simplici, cylindrica, obtusa; striis transversis tenuibus.

Münsteria vermicularis Stenne. Vers. Flor. Vorw. II. pag. 32. tab. 1. fig. 3. - Endr. Gen. plant. Suppl. III. pag. 5ร. - UNG. Conspect. Flor. primord. in Chlor. Protog. pag. xxvi.; Gen. et Spec. plant. foss. pag. 14. - Bronn Ind. Palaeont. P. I. pag. 746. - Brosc. Tabl. gen. végét. foss. pag. 105. - Greber Deutschl. Petrefact. pag. $8, n_{\circ}^{\circ} 58$.

\section{Eodem in loco cum priore.}

Questa specie ha la fronda semplice eretta, tubulare, cilindrica. E lunga sette centimetri, oltusa e rotondata tanto nell' estremita inferiore che nella superiore. Verso la base ha un diametro di nove millimetri, che si conserva eguale per due terzi della sua lunghezza, e poi gradatamente va scemando, fino a che nel terzo superiore si riduce a cinque. Ivi mostra lieve ondulazione nel suo contorno, e si volge da un lato con curva appena sensibile. Essa è percorsa trasversalmente da strie interrotte assai più marcate di quelle della specie precedente.

Sulla figura data dallo Sternberg non si scorge alcuna traccia di que’ sporangi puntiformi di colore ranciato, di cui egli fa cenno.

\section{DIünsteria Iacunosa Sternb.}

M. fronde solitaria simplici, cylindrica, obtusa, incurva; striis transversis vel obliquis confluentibus, lacunoso-rugosis.

Münsteria lacunosa Sterne. Vers. Flor. Vorw. II. pag. 52. tab. 1. fig. 4. - End. Gen. plant. Suppl. III. pag. 55. - UNG. Conspect. Flor. primord. in Chlor. Protog. pag. xxvi.; Gen. et Spec. plant. foss. pag. 14. - Brons Ind. Paleont. P. I. pag. 746. - Brong. Tabl. gen. végét. foss. pag. 105. - Greber Deutschl. Petrefact. pag. 8, n. $^{0} 59$.

Gum priore ad Solenhofen.

Alga di colore bruno-ranciato, colla fronda semplice, lunga poco più di quattro centimetri e larga all'incirca otto millimetri, ottusa in ambe le estremità e alcun poco curvata nel mezzo. Essa è irregolarmente percorsa in senso obbliqquo da rughe assai rilevate, avvicinate e spesso confluenti, e da solchi trasversali disposti senza ordine, che in più punti $s^{p}$ intersecano. 


\section{$-14-$}

Da quanto ci palesa l'esemplare figurato dallo Sternberg, sarei indollo a ritenere che non avesse appartenuto ad un individuo giunto al suo pieno accrescimento.

\section{Gen. WH. Hazmentes Sternb.}

Frons coriacea vel submembranacea, plana vel fistulosa. Sporangia tuberculiformia vel punctiformia, laminae frondis immersa.

Sterix. Vers. Flor. Vorw. II. pag. 29. - Endu. Gen. plant. Suppl. III. pag. 54. Ung. Conspect. Flor. primord. in Ghlor. Protog. pag. xxvir.; Gen. el Spec. plant. foss. pag. 21. - Bronn Leth. Geogn. edit. 3. fasc. 2. pag. 43.

Fucoides, $\mathbb{S}$ 5. Gigartinites Brong. Prodr. pag. 20.; Hist. des végét. foss. I. pag. 56 (pro parte).

Sphaerococcites Brokra. Tabl. yen. végét. foss. pag. 10 et 103 (pro parte).

\section{Halymenites vermiculatus Sternb.}

H. fronde plana, diffusa, flexuosa, irregulariter bipinnatim ramosa, ramis ramulisque obtusis dentatis.

Halymenites vermiculatus Sternis. Vers. Flor. Vorw. II. pag. 29. tab. 5. fig. 5. - ENDL. Gen. plant. Suppl. III. p. 54. - UNG. Conspect. Flor. primord. in Chlor. Protog. pag. xxvir.; Gen. et Spec. plant. foss. pag. 21. - Brons Ind. Palaeont. P. I. pag. 568. - G GEbeL Deutschl. Petrefact. pag. 11, n. ${ }^{\circ} 94$.

Sphaerococciles vermiculatus Trevis. $1849 \mathrm{in} \mathrm{mss.} \mathrm{edit,} \mathrm{II.} \mathrm{Nomencl.} \mathrm{Algarum.}$

In calcareo lithographico ad Solenhofen.

Lo Sternberg, descrivendo quest'Alga, cita la tavola 8. fig. 3. della sua opera, e questa cilazione venne dopo di lui ripetuta da tutti gli altri. Ma questa figura non presenta alcuno dei caratteri indicati dalla frase specifica, e corrisponde invece perfettamente alla descrizione della Münsteria flagellaris (Sternb. Flor. Vorw. II. pag. 32), cui dovrà quindi innanzi essere riferita.

L' ispezione dell' esemplare illustrato dallo Sternberg, e che si conserva nell' I. R. Museo di Praga, basta a convincere che questa specie è invece rappresentata dalla fig. 3. tavola 5. Vol. II. della Flora der Vorwelt.

IIo lasciato quest' Alga nel genere Halymenites, in cui fu collocata dallo Sternberg, e ritenuta da Unger, Bronn e Giebel, sebbene, a dir vero, non abbia tutti i caratteri assegnati a questo genere dallo Sternberg stesso. 


\section{Malymenites cactiformis Sternb.}

H. fronde fistulosa dichotoma, creberrime punctata, ramis crassis inaequalibus obtusis, medio crassioribus.

Halymenites cactiformis Stenxi. Vers. Flor. Vorw. II. pag. 29. tab. 2. fig. 2. - End. Gen. plant. Suppl. III. p. כّ4. - UxG. Conspect. Flor. primord. in Ghlor. Protog. pag. xxvir.; Gen. et Spec. plant. foss. pag. 21. - Bronn Ind. Palacont. P. I. pag. \$̋ั4. - Gieber Deutschl. Petrefact. pag. 11, n. ${ }^{\circ} 95$.

Sphaerococcites cactiformis BRovg. Tabl. gen. végét. foss. pag. 105.

Eodem in loco cum priore.

Il nome specifico dato a questa specie dallo Sternberg risponde assai bene all' aspetto che presenta. La fronda si divide per dicolomia in alcuni rami di apparenza crassa, il cui diametro a vicenda si allarga e si ristringe, simulando cosi appunto in qualche modo le articolazioni delle Opunzie.

Tutta la fronda raggiunge una lunghezza di circa undici centimetri, ed i rami che la compongono variano assai nelle loro dimensioni. Alcuni giungono ad avere nel tratto piì allargato due centimetri di diametro, ed uno solo nei punti piu angusti; altri all'incontro sono della metà più piccoli. Generalmente il diametro dei punti, ove si ristringe il ramo, sta, a quello delle porzioni più larghe, all’ incirca come uno sta a due.

Tutta la pianta è fittamente seminata di punti brunicci tondeggianti e bene distinti, $e$ le estremità dei rami e dei ramoscelli terminano in punta ovato-lanceolata assai ottusa.

\section{Halymenites varius sternb.}

H. fronde fistulosa dichotoma punctatissima, ramis cylindricis obtusis hine inde sinuato-dentatis, aliis elongatis rectis, aliis brevioribus digitatis.

Halymeniles varius Sterne. Vers. Flor. Vorw. II. pag. 29. tab. 2. fig. 4. - Ende. Gen. plant. Suppl. III. pag. 5ै4. - UrG. Conspect. Flor. primord. in Ghlor. Prolog. pag. xxvir.; Gen. el Spec. plant. foss. pag. 21. - BRоNN Leth. geogn. pag. 43. tab. 14. fig. 3.

Sphaerococcites varius BRonG. Tabl. gen. végét. foss. pag. 10 כ.

Eodem in loco cum prioribus ad Solenhofen.

La fronda, tulta cospersa di punti minuti e bene marcali, si dicotomizza in rami piu o meno cilindroidi, che variano nel diametro dai tre ai sette millimetri, alcuni de quali 


\section{$-16$}

divergono ad angolo retto o leggermente aculo, prolungandosi dai quattro ai cincue centimetri; altri più brevi sembrano quasi mozzi, e non superano in lunghezza i sedici millimetri. Questi ultimi si espandono dalla porzione piì larga della fronda in forma digitata, e nell' esemplare figurato dallo Sternberg sono in numero di cinque.

Tutte le parti, in cui si divide o ramifica la fronda, terminano ottusamente e tondeggianti in punta, e si mostrano nei lati quà e là sinuato-dentate, cos denti minuti poco marcati, e distanti fra di loro.

La figura data dal Bromn nella Lethaea è alquanto diversa da quella dello Sternberg. La fronda vi comparisce piuttosto piana che fistolosa, nè vi sono tracciale le punteggiature, che d'altronde sono bene espresse nella figura della Flora der Vorvelt.

\section{Halymenites subarticulatus Sternb.}

H. fronde fistulosa dichotoma punclatissima, ramis geniculato-divaricatis medio constrictis, internodiis rentricosis, ramulis clavalis, in apice ramorum digitatis.

IIalymenites subarticulalus Sterne. Vers. Flor. Vorw. II. pag. 29. tab. 4. fig. 2. Exde. Gen. plant. Suppl. III. pag. 55. - Uvg. Conspect. Flor. primord. in Chlor. Protog. pag. xxvir.; Gen. et Spec. plant. foss. pag. 22. - Brons Ind. Palacont. P. I. pag. 56 د. Greber Deutschl. Petrefact. pag. 12, n." 97.

Sphacrococcites subarticulatus Brosk. Tabl. gen. végél. foss. pag. 10 ว̆.

Cum prioribus ad Solenhofen.

Questa specie deve avere avuto una fronda piultosto allungata, essendoché nell' esemplare, di cui lo Sternberg ci diede la figma, supera in lunghezza i sedici centimetri senza che se ne possa scorgere il termine, per essere rimasto mozzo l'esemplare verso l'estremità superiore.

La base della fronda ha sei millimetri di diametro, e dopo brevissimo tratto si ristringe; indi torna a farsi piu larga, ove un rano claviforme, lungo due centimetri, n’esce lateralmente ad angolo quasi retto, ed altro alquanto maggiore dal lato opposto si espande ad angolo acuto. Poi si fa strelta, riducendosi a poco più di tre millimetri, e scorre cosi per ollo millimetri fino al punto ove si piega bruscamente ad angolo retto. Ivi si allarga in forma obovato-bishunga, ragggiungendo, sopra quattro centimetri di lunghezza, una larghezza di quindici millimetri. Rapidamente da questa discende di nuovo ai tre millimetri di diametro; indi torna ad espandersi, lasciando scorgere da un lato breve rudimento di un ramo; prosegue per altri cinque centimetri, dilatandosi nuovamente fino a riavere il diametro di quindici millimetri; e termina agli orli dell' esemplare suddividendosi in forma digitala. 
Tali notabili variazioni del diametro danno a questa specie l'apparenza di essere pârtita in più arlicolazioni.

I punti minuti e spessi, da cui è segnata, non sono da per tutto cosi bene marcati e chiaramente visibili, come nelle altre specie fistolose di questo genere.

\section{Halymenites secundus Sternb.}

H. fronde fistulosa dicholome-tripinnalim ramosa, punctatissima, ramis secundis teretibus aequilatis ramulisque obtusis.

Halymenites secundus Stenne. Vers. Flor. Vorw. II. pag. 29. tab. 4. fig. 5. - Exds. Gen. plant. Suppl. III. pag. 54. - UNG. Conspect. Flor. primord. in Chlor. Protog. pag. xxvir.; Gen. et Spec. plant. foss. pag. 22. - Bronn Ind. Palaeont. P. I. pag. 565. - Greber Deutschl. Petrefact. pag. 12, n. $^{\circ} 98$.

Sphaerococeites? secundus Broria. Tabl. gen. végét. foss. pag. 10 ร.

Gum prioribus ad Solenhofen.

Una fronda tripimnata nelle ramificazioni inferiori, lunga all'incirca setle centimetri, colla base molto allargata, e dalla cui porzione centrale si diramano molti ramoscelli rivolti per lo più da un lato, caratterizzano questa distintissima specie. I rami, ch's escono lateralmente dalla base, piegano un poco all’ ingiù, e si ramificano suddividendosi assai piu minulamente di quelli che sorgono dalla parte opposta e dal tratto superiore della fronda, la quale corre per tre centimetri, mantenendo il diametro di tre millimetri. Questa porzione della fronda è munita da un lato solo di rami grossi due millimetri, ed in cima si divide in quattro ramificazioni $d^{\prime}$ eguale spessore. Da tutti questi rami sporgono più ramoscelli assai corti, tronchi all’estremità, rare volte claviformi. Tutta la pianta è uggualmente seminata di minutissimi punti.

\section{Halymenites Schnitzleinii Sternb.}

H. fronde fistulosa punctatissima, basi tenui tereti, hinc ventricosa, illine angustior, ramosissima, ramulis linearibus flexuosis obtusis.

Halymeniles Schnitzleinii Sterne. Vers. Flor. Vorw. II. pag. 30. tab. VI. fig. 1. - Exdr. Gen. plant. Suppl. III. pag. 5\%. - UNG. Gonspect. Flor. primord. in Ghlor. Protog. pag. xxvir; Gen. et Spec. plant. foss. pag. 22. - Brown Ind. Palaeont. P. I. pag. כ̌6̆. - GIeber Deutschl. Petrefact. pag. $13,{ }^{\circ} .^{\circ} 99$.

Sphaerococcites Schnitzleinii Broxg. Tabl. gen. végét. foss. pag. 10 .

Cum prioribus ad Solenhofen. 
Questa specie si distingue per avere una fronda che nella parte inferiore giunge appena a tre millimetri di diametro, e superiormente si espande fino ai due centimetri; indi si ristringe a cinque millimetri, poi torna ad allargarsi alcun poco nella porzione terminale.

Dagli orli di essa sporgono per ogni verso dei rami più o meno dilatali, di cui i più larghi si riuniscono fra loro; i più sottili, liberi, flessuosi, sono talvolta dicotomi.

La superficie in ogni sua parte è finamente punteggiata, ed ottuse terminano tutte le estremilà dei rami.

Anche qui conviene notare un errore esistente nell' opera dello Sternberg. In essa, alla pagina 30 , si cita la fig. 1. della tavola 5.; ma questa figura rappresenta invece una di quelle Caulerpiti che ora hanno preso posto tra le Conifere nel genere Arthrotaxites di Unger. Nel Genera et Species plantarum fossilium è ripetuto il medesimo abbaglio mentre all’Halymenites Schnitzleinii corrisponde perfeltamente la figura 1. della tavola 6.

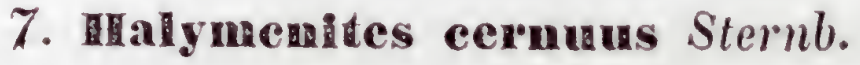

H. fronde fistulosa punctatissima, a basi dichotoma, ramis teretibus obtusis, arcuato-divaricatis, hinc latioribus, illine angustioribus, simplicibus ramulosisque, ramulis conformibus.

Halymenites cernuus Sterna. Vers. Flor. Vorw. II. pag. 50. tab. 8. fig. 4. - Ende. Gen. plant. Suppl. III. pag. 54. - UNG. Conspect. Flor. primord. in Chlor. Protog. pag. xxvir.; Gen. et Spec. plant. foss. pag. 22. - Brown Ind. Palaeont. P. I. pag. 56\%. - Gieber Deutschl. Petrefact. pag. $12, \mathrm{n} .{ }^{\circ} 100$.

Sphaerococcites cernuus Broxg. Tabl. gen. végét. foss. pag. 10 əั.

Eodem in loco cum prioribus.

La fronda è fistolosa, ed ha una base dilatata, che si dicotomizza in più rami divaricati, arcuati, dicotomi, aventi un diametro ineguale, perchè in alcuni punli s'allarga, in altri si ristringe, e solo verso la base si mantiene per breve tratto di cinque millimetri all ${ }^{2}$ incirca.

Tulta la superficie è minutamente segnata di punti, che nella porzione più larga della base sono più rilevati; e gli orli dei rami e dei ramoscelli si mostrano per intervalli or'a lisci, ora un $\mathrm{po}^{5}$ scabri. Le estremità sono tutte bislunghette, ed ottuse all’apice.

Non è certo a questa specie che può essere riferita nella Flora der Vorwelt la figura 4. della tavola 7., come viene erroneamente indicato dallo Sternberg, e dopo di lui 
da altri; ma bensi la figura 4. della tavola 8., che rappresenta esattamente l'esemplare segnato col n. ${ }^{\circ} 401$ nella Collezione di Praga.

\section{Dlalymenites Stockesii Ung.}

H. fronde fistulosa dichotome-tripinnatim ramosa, ramis ramulisque patenlissimis, cylindraceis, inaequalibus, obtusis, rugosis, subtuberculatis.

Halymenites Stockesii UNG. Gen. et Spec. plant. foss. pag. 22. - Giebed Deutschl. Petrefact. pag. 12, n. ${ }^{\circ} 101$.

Halymenites Stockii Sterni. Vers. Flor. Vorw. II. p. 50. - Endu. Gen. plant. Suppl. III. pag. 5ั. - Ung. Conspect. Flor. primord. in Chlor. Protog. pag. xxvIr.

Fucoides Stockii Brong. Hist. végét. foss. I. pag. 61. tab. 6. fig. 5. 4.; Prodrom. pag. 20.

Sphaerococcites Stockei Bronn Ind. Palaeont. P. I. pag. 565.

Sphaerococcites Stockii Bnovg. Tabl. gen. végét. foss. pag. 103.

Eodem in loco cum prioribus.

Questa specie ha la fronda lunga otto centimetri, fistolosa, cilindroide-compressa, che irregolarmente si divide in ramoscelli più fiate dicotomi, per alcun breve tratto talvolta pinnati, e sempre oltusi nelle estremità. Ne è vario il diametro, che però non sorpassa i tre millimetri. Taluno de rami terminali si mostra rigonfio, arcuato, e colla superficie rugoso-tubercolosa. Quest'apparenza è bene rappresentata dalla fig. 4. deila tav. 6. nell'Histoire des végétaux fossiles dell'illustre Brongniart.

Dedicata dal Brongniart al sig. Stockes (e non Stock), possessore dell' esemplare che descrisse e figurò nell'opera testè citata, conviene ritenere il nome specifico, come fu emendato nel Genera et Species plantarum fossilium di Unger.

\section{Malymenites Goldfussil Sternb.}

H. fronde fistulosa cylindrica, granulato-punctatissima, medio tripartila, ramis simplicibus, oppositis, cylindricis, apice attenuatis, medio majore.

IIalymenites Goldfussii Sterne. Vers. Flor. Vorw. II. pag. 50. - End. Gen. plant. Suppl. III. pag. 55. - UNG. Conspect. Flor. primord. in Chlor. Protog. pag. xxvir.; Gen. et Spec. plant. foss. pag. 22. - Brown Ind. Palaeont. P. I. pag. 565 ; Leth. Geogn., edit. 3. fasc. 2. pag. 45. - Greber Deutschl. Petrefact. pag. 12, n. ${ }^{\circ} 102$.

Achilleum dubium GoLdr. Petrefact. I. pag. 1. tab. 1. fig. 2.

Trioxites rugosa Rarinesque Bull. Soc. Géol. Fran. 1839. Vol. X. pag. 580. 
Gum prioribus ad Solenhofen.

La fronda di questa specie è lunga all’ incirca undici centimetri. Nella parte inferiore per tre centimetri si mantiene larga dai dieci ai dodici millimetri, e mostra lateralmente due espansioni brevissime, che sembrano rudimenti di rami. Al di sopra e quasi a mezzo della lunghezza della fronda sorgono, uno per parte, due rami un po curvi, e diretti ver'so $l^{2}$ apice; $\mathbf{i}$ quali, lentamente decrescendo, si fanno acuminati nella loro estremità. La porzione centrale della fronda oltrepassa di tre centimetri questi rami laterali, e termina anch' essa in punta, gradatamente diminuendo nel diametro.

La superficie è resa scabra dalle fitte granulazioni che la ricoprono.

\section{Halymenites Hrongniartii Sternb.}

H. frondibus fistulosis in coespilem aggregatis, elongato-clavatis, obtusis, crebre granulato-punctatis.

Halymenites Brongniartii Sterne. Vers. Flor. Vorw. II. pag. 30. - Endu. Gen. plant. Suppl. III. pag. эั5. - UNG. Conspect. Flor. primord. in Ghlor. Protog. pag. xxvir.; Gen. et Spec. plant. foss. pag. 22. - Bronn Ind. Palaeont. P. I. pag. 564. - Ginber Deutschl. Petrefact. pag. $12, \mathrm{n} \cdot{ }^{\circ} 105$.

Fucoides encoelioides Brovg. Hist. végét. foss. I. pag. 5̌. tab. 6. fig. 2. (exclus. fig. 1.)

Gum prioribus ad Solenhofen.

Il carattere essenziale di questa specie consiste nella costante riunione di più frondi a guisa di cespo, e nell' essere queste configurate in forma di clava un po allungata, senz'alcuna traccia di strie, e fittamente seminate di tubercoletti o granelli disposti con cerla simmetria e molto rilevati.

Lo Sternberg e l’Unger attribuiscono a questo Halymenites la fig. 2. della tavola 6 . dell' Histoire des régétaux fossiles di Brongniart, mentre invece riferiscono la figg. 1. alla Münsteria encoelioides di Bronn ( $M$. clasata Sternb.). Infatti quella figura 2. non mostrando alcuna traccia delle strie trasversali caratteristiche del genere Münsteria, escluderebbe il sospelto che in essa sia rappresentata una specie di questo; e neppure nella descrizione del Fucoides encoelioides, alla pag. 55 dell'opera citata, è fatta parola alcuna di simili strie. $\mathrm{Ma}$, d'altra parte, osserviamo che lo stesso Brongniart, nel più volte encomiato suo Tableau des genres des végétaux fossiles (pag. 105), ammettendo fra le specie del genere Münsteria la $M$. clavata, si astenne dall'annoverare fra i suoi Sphaerococcites l'Halymenites Brongniartii. Quindi ogni, forse solo apparente, incertezza su 
ciò non può essere tolta se non dalla ispezione degli esemplari della collezione del signor Stockes, sopra i quali il Brongniart ha fondata la sua specie. Del resto, sembrerebbe che la sola differenza fra le due piante denominate dallo Sternberg Münsteria clavata ed Halymenites Brongniartii si riduca alla presenza nell’ una, ed alla mancanza nell’altra, delle tenuissime strie trasversali.

\section{Halymenites concatenatus Sternb.}

H. fronde fistulosa dichotoma, punctatissima, ramis crassis, cylindraceis, concatenatis, hinc inde sinuato-dentatis, basi attenuatis.

Halymenites concatenatus Sxenne. Vers. Flor. Vorw. II. pag. 50. tab. 2. fig. 1. a. b. Endu. Gen. plant. Suppl. III. pag. 5̋. - Ung. Conspect. Flor. primord. in Chlor. Protog. pag. xxvir.; Gen. et Spec. plant. foss. pag. 25. - Brown Ind. Palaeont. P. I. pag. Ј̌6̆̀. Gieber Deutschl. Petrefact. pag. 12, n. ${ }^{0} 104$.

Sphaerococcites coneatenatus Brova. Tabl. gen. végét. foss. pag. 10 əั.

Cum prioribus ad Solenhofen.

La fronda larga, fistolosa, dicoloma di questa specie si distingue assai da tulte le altre per la forma concatenato-reticolata dei rami, che ora si accavalcano, ora si anastomizzano l'uno con l'altro, lasciando fra loro degli spazj vuoti triangolari. Nell' esemplare figurato dallo Sternberg ciò si scorge specialmente nella parte inferiore; superiormente un ramo lungo tre centimetri si espande obbliquamente da un lato; un altro s'erge con lieve curva, e s'allunga per cinque centimetri dal lato opposto. Entrambi hanno alla base un diametro di otto millimetri all’ incirca, e s'allargano fino ai quattordici verso le estremilì, che sono tronche. I rami inferiori variano nel diametro dai cinque ai dieci millimetri, $\mathrm{e}$ si uniscono dopo breve tratto fra loro ad angolo acuto. La fronda, seminata di punti minutissimi, riesce alquanto scabra, e gli orli ne appariscono sinuato-dentati.

Nella fig. $1 . b$ dello Sternberg è effigiato il rovescio della fronda.

\section{Halymenites ramulosus Sternb.}

H. fronde fistulosa dichotoma, punctatissima, cylindracea, ramis angulo aculo divergentibus cylindricis, apice in ramulos breves ovatos lobiformes divergentes divisis.

Halymenites ramulosus Sterne. Vers. Flor. Vorw. II. pag. 51. - Morris Cat. Brit. foss. pag. 9. - Endu. Gen. plant. Suppl. III. pag. 5马. - UNG. Gonspect. Flor. primord. in Ghlor. Protog. pag. xxvir.; Gen. et Spec. plant. foss. pag. 23. - Bronn Ind. Palaeont. P. I. pag. 66 כ. 
Fucoides furcatus, var. ß. ramis majoribus brevioribus apice vix inerassatis Brosa. Hist. végét. foss. I. pag. 62. tab. 3. fig. 2.

Sphacrococcites ramulosus Brong. Tabl. gen. végét. foss. pag. 105.

In calcareo scissili formationis oolithicae inferioris ad Stonesfield prope Oxoniam Angliae.

Quest ${ }^{2}$ Alga si presenta sotto $\mathrm{l}^{2}$ aspetto di una fronda cilindroide-compressa, avente um diametro di sei millimetri, fortemente incurvata alla base, poi raddrizzata alquanto, e per un tratto di quattro centimetri priva di rami. Si divide poscia in due ramificazioni divergenti, larghe quanto la parte inferiore della fronda. Una di queste viene troncata dal$l^{9}$ orlo dell'esemplare; l'altra si prolunga alcun poco, indi si pârte in due espansioni oroidee, pure divergenti.

La superficie anche di questa specie è tutta ricoperta da granulazioni ben distinte.

\section{Gen. HX. Bastocanpites Trevis.}

Frons coriacea, cylindrica, fistulosa?, simplex, oblonga, rugosa, papillis elongatis ornata. Sporangia verucaeformia, puncto centrali instructa, in disco frondis sparsa.

Trevis. 1849 in mss. edit. II. Nomencl. Algarum.

\section{Mastocarpites erucaeformis Trevis.}

M. fronde oblonga, obtusa, incurva, rugosa, papillis elongalis, sporangiisque verrucaeformibus sparsis ornala.

Naslocarpites erueacformis Trevis. 1849 in mss. edit. II. Nomencl. Algarum.

Algacites erucaeformis Sternb. Vers. Flor. Vorw. II. pag. 56. tab. 2. fig. 5. 6. - Ung. Gen. et Spec. plant. foss. pag. 51. - Brosn Ind. Palaeont. P. I. pag. 2 כ. - Gieber Deutschl. Petrefact. pag. 1 , n. n. 156 .

\section{In calcareo scissili ad Solenhofen.}

Fronda semplice, cilindroide, che sembra fistolosa, lunga tre centimetri, larga uno e mezzo, bislunga, rotondata in ambe le estremità, curva nel mezzo. La superficie rugosoverucosa è coperta di papille allungate, e sul rovescio (S SеRnв. loc. cit. fig. 6.) lascia vedere da un lato una porzione seminata di minulissimi punti neri. I tubercoletti (sporangi?), che si veggono sparsi quà e là, sono rotondi, rilevati, e con un punto nero nel centro. Alcuni fra essi raggiungono il diametro di un millimetro. 


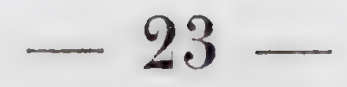

Sternberg ed Unger stanno dubbiosi se quest'Alga si debba collocare fra le Münsterie, o nel genere Halymenites. Però nella figura data dal primo di questi autori non $\mathrm{v}^{5} \mathrm{ha}$ traccia delle strie trasversali caratteristiche del genere Münsteria; mentre vi sono all’ incontro chiaramente espressi i tubercoli rilevali e le papille allungate, che eerto non formano parte dei caratteri assegnati dallo stesso Sternberg al genere Halymenites. Per questi motivi non esitai ad abbracciare $l^{2}$ opinione del Trevisan, il quale scorge molta analogia esistere fra quest' Alga ed alcune specie viventi riunite dal Kützing solto il nome generico di Mastocarpus, e collocate nella famiglia delle Gigartinee (Kützrsa, Species Algarum, pag. 732), nella quale sono pure ritenute da Jacopo Agardh nella diligentissima sua opera, in corso di stampa, intitolata Species Genera et Ordines Algarum.

\section{Gen. X. Trevisava Zign.}

Frons cartilaginea, teres, fistulosa, dichotoma, tubo diaphragmalibus intercepto. Sporangia inconspicua.

\section{Trevisania farcellada Zign.}

Tar. 1. fig. 4.

T. fronde tereli-filiformi, rigidiuscula, pluries dicholoma, ramis patenlibus aequilatis obtusiusculis.

In calcareo griseo formationis oolithicae inferioris ad Val d'Assa prope Rotzo in Septem Communibus Provinciae Vicetinae.

Questo nuovo genere si dislingue essenzialmente dagli altri per avere la fronda fistolosa ed internamente divisa da diaframmi (fig. 4. a). Esso è intitolato al distinto botanico Cav. Viltore Trevisan, che mi ha comunicate con somma liberalità parecchie interessanti sue osservazioni, tultora inedile, sulle Alghe fossili.

La fronda, nel nostro saggio, si dicotomizza regolarmente sino a tre volte, variando la distanza fra una dicolomia e l'altra dai quindici ai quaranta millimetri. Essa è di apparenza piuttosto consistente, ed ha il diametro di circa un millimetro, che si mantiene eguale anche nei rami, i quali sono patenti e cogli apici ottusi. Le porzioni visibili, nell'esemplare di cui porgo la figura, giungono a dodici centimetri di lunghezza; ma siccome nella parte inferiore non' se ne scorge il termine, cosi non possiamo ritenere questa misura come rappresentante la totale estensione della pianta. I setli o diaframmi, che nelle porzioni inferiori della fronda sono ben marcati e discosti l' uno dall' altro appena un millimetro, scorgonsi nelle parti superiori meno chiaramente visibili e più distanti. 


\section{$-24-$}

Il predominio dei resti vegetali spettanti a piante terrestri, e la finora rimarcata mancanza di altre Floridee nel banco fitolitifero dell'Oolite inferiore delle Alpi Venete, mi hanno fatto da prima esitare sulla Classe a cui dovessi riferire questa pianticella.

A primo aspelto essa ricorda il Chondrites Solenites dell' Unger, ossia la Solenites? furcata di Lindley ed Hutton (Foss. Flor. of Great Brit. Vol. III. tab. 209.), specialmente pel modo con cui si ramifica. L'apparente consistenza del tessuto, e la regolare divisione della fronda in rami dicotomi, potevano in certo modo giustificare il sospetto che la pianta di Rolzo fosse analoga al genere Psilotum dell? epoca attuale, come per la fronda fistolosa ed internamente spartita da diaframmi sembrava avvicinarsi eziandio alla Solenites Murrayana (Linds. and IIutr. Foss. Flor. of Great Brit. vol. II. tab. 121), posta dall' Unger nel genere Isvetites (Gen. et Spec. plant. foss. pag. 226). Ma l'Isoetites Murvayana ha le foglie semplici, nè giammai si dicotomizzano quelle delle altre specie, sia spente, sia viventi, che appartengono alla famiglia delle Isoetacee. Tanto poi le Alghe Cossili comprese nel genere Chondrites, quanto le Licopodiacee virenti del genere Psilolım, non sono fistolose, nè quindi porgono traccia alcuna dei diaframmi che si osservano nella nostra pianta; nella quale, $d^{2}$ altronde, manca del pari ogni vestigio delle foglie minulissime, setiformi, sparse, proprie dei Psiloti conosciuti.

Per questi caratteri, che molto ricordano quelli assegnati dagli autori al vivente genere Champia (Desv. in Lamovr. Ess. sur les Thalass. pag. 51 ) di Floridee ("Frons " cylindracea aut compressa, ramosa, tubulosa, tubo diapliragmatibus intercepto " J. G. A GARdi Spec. Gen. et Ord. Algar. Vol. II. P. 2. pag. 369), mi trovai indotto a collocarla fira le Alghe di quest' Ordine.

\section{Gen. X甘. Cuonorites Sternb.}

Frons cartilaginea, filiformis, dichotome ramosa, ramis cylindricis, in ectypis compressis.

Sterib. Vers. Flor. Vorw. II. pag. 25. - Göpp. System. Filic. foss. pag. 268. - End. Gen. plant. Suppl. III. p. 55. - Ung. Conspect. Flor. primord. in Chlor. Protog. pag. xxvir.; Gen. et Spec. plant. foss. pag. 15. - Broxa. Tabl. gen. végét. foss. pag. 9. - Massaz. Schizz. geogn. sulla Valle del Progno, pag. 32. - BRoNn Leth. geogn. edit. 5. fasc. 2. pag. 42.

Fucoides $\ 5$. Gigartinites Brong. Prodrom. pag. 20.; Hist. végét. foss. I. pag. 56.

\section{Chondrites Iaxus Sternb.}

Ch. fronde tripinnatim ramosa, ramis sparsis recurvato-pendulis, ramulispue filiformibus acutis. 


\section{$-2 \breve{3}-$}

Chondrites laxus Sterne. Vers. Flor. Vorw. II. pag. 27, tab. 24. fig. 1. - Ende. Gen. plant. Suppl. IIT. pag. 5̋. - UNG. Conspect. Flor. primord. in Ghlor. Prorog. pag. xxvir.; Gen. et Spec. plant. foss. pag. 18. - Bronn Ind. Palaeont. P. I. pag. 294. - Brong. Tabl. gen. végét. foss. pag. 10อ̈. - Gieber Deutschl. Petrefact. pag. 10, n. 76.

Fucoides pendulinus Münster in litt. ad $\mathbf{S}_{\text {Termb. }}$

In calcareo lithographico ad Solenhofen.

La fronda per un tratto di tre centimetri e mezzo si mantiene eretta e larga circa quattro millimetri, con lieve ristringimento nel mezzo; sotto del quale sorge un ramo che si scosta lateralmente ad angolo acuto, e progredisce all' insù per tre centimetri; indi si piega e volge al basso, suddividendosi in piu ramoscelli pinnati, gli ultimi dei quali sono brevissimi ed acuminati. La porzione più grossa della fronda termina pârtendosi in tre rami flessuosi, semplici per breve tratto, poi tripinnati, rivolti dopo lieve curva al$l^{2}$ ingiù, e $\mathrm{co}^{\mathrm{P}}$ ramoscelli terminali pendenti, filiformi ed aculi.

Questa specie fu rinvenuta dall'illustre naturalista il Conte di Münster nella calcaria scissile litografica di Solenhofen, e da esso commicata allo Sternberg solto il nome di Fucoides pendulinus, col quale volle esprimere il carattere particolare presentato dalle sue ramificazioni, che $\mathrm{s}^{2}$ incurvano e pendono colle estremità rivolte al basso da ambo i lati della fronda. Il nome datole dallo Sternberg caralterizza però assai meglio l'aspetto generale di questa pianta.

\section{Chondrites Solenites Ung.}

Ch. fronde stipitata pluries dichotoma, ramis divaricatis obtusis, stipiteque teretibus, linearibus, aequilatis, rectis, longitudinaliter obsolete striatis.

Chondrites Solenites Uvg. Conspect. Flor. primord. in Ghlor. Protog. pag. xxvir.; Gen. et Spec. plant. foss. pag. 19.

Solenites? furcata Lindu et Hutt. Foss. Flor. of Great Brit. Vol. III. tab. 209. - Morris Cat. of Brit. Foss. pag. 21. - Bronn Ind. Palaeont. P. I. pag. 1156 (exclus. synon.). Brosg. Tabl. gen. végét. foss. pag. 38 et 41.

Chondriles furcatus Prest in Sterne. Vers. Flor. Vorw. II. pag. 103 (non Chondrites furcatus Sterni. loc. cit. pag. 27).

Baiera furcata Braun in Münster Beitr. zur Petrefacten-kunde. Fasc. VI. pag. 21.

In arenaria inferiori formationis oolithicae ad Haiburn Wyke prope Scarborough in Anglia. 
Questa pianticella, lunga all' incirca nove centimetri, ha nella parte inferiore un diametro di due millimetri, e per un tratto di due centimetri si mostra affatto priva di rami. Poscia si dicolomizza, assottigliandosi e suddividendosi collo stesso sistema in più ramoscelli, che si scostano alquanto $l^{2}$ uno dall'altro dopo le inforcature, e finiscono ottusamente tronchi nelle estremità. La lunghezza di questi ramoscelli varia dai dieci ai quaranta millimetri, ed il loro diametro giunge appena ad un millimetro. La superficie ne è longitudinalmente segnata da minutissime strie, visibili soltanto in alcuni punti dell'esemplare.

Accennai poco fa alla simiglianza che passa fia la mia Trevisania furcellata e la

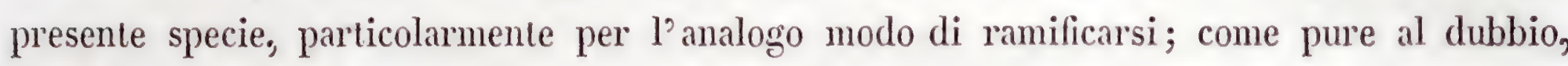
in me risvegliato da questo carattere, che la prima potesse appartenere al vivente genere Psilotum. La mancanza delle foglie, la fistolosità della fronda, e i diaframmi che intersecano la Trevisania, mi fecero abbandonare questo sospetto, che vediamo essersi egualmente destato nel Brongniart riguardo alla specie di cui qui si tratta. Infatti parlando, nel Tableau des genres des végétaux fossiles, alla pagina 41 , del genere Psilotites, cosi questi si esprime: "Le Solenites? furcata de Lindley et Hutton (Foss. Flor. n. ${ }^{\circ} 209$ ), ") du terrain oolithique de Scarborough, a beaucoup $1^{2}$ aspect des tiges dichotomes des „Psilotum; mais on $\mathrm{n}^{9} \mathrm{y}$ a pas observé ces feuilles rudimentaires, qui indiqueraient clai" rement sa nature caulinaire, et on peut aussi admettre que $c^{9}$ est une feuille dichotome, " comme celle des Baiera ou des Schizopteris."

A questo proposito giova rammentare come il Prof. Braun fino dal 1844 avesse appunto annoverata la Solenites? furcata fra le specie del suo nuovo genere Baiera con le seguenti parole: "Lindley's und Hutton's Solenites? furcata möchte ich gleichfalls " der Gattung Baiera zuzahlen, originale exemplare hatte ich leider zu untersuchen keine " Gelegenheil, die Uebereinstinnung der Gattungs-charaktere aber kann nur dadurch sicher " dargethan werden, habiluelle analogie spricht jedoch ganz dafür. ") (BRAun in Müsst. Beitr. Fasc. VI. pag. 20). Ma, a dir vero, è ben difficile trovare una qualche analogia fra questa impronta ed il genere Baiera, che dal suo autore è caratterizzato come segue: "Venae primariae frondis pluries dichotomae; venae secundariae ac venulae in areas s) hexagonales elongatas irregulariter confluentes. Sporocarpia capsulaeformia, oralia, pe"dunculata, ternata vel biternata." Nulla di tutto ciò si osserva nella Solenites? furcata, e quindi rettamente osservò il Brongniart (Tabl. genr. végét. foss. pag. 38), tratlando del genere Baiera, che "quant au Solenites? furcata de Lindley et Ilutton, sa forme "générale est si differente, qu’il me parait difficile de le classer dans le même genre."

Riguardo poi al genere Schizopteris di Brongniart, la pianta di cui tratliamo non vi può per certo essere riferita, non presentando essa alcuno dei caratteri assegnatigli. 


\section{7}

Per queste ragioni fui indotto ad abbracciare l'opinione di Presl, di Göppert, di Bronn e di Unger, i quali tutti collocano la Solenites? furcata tra le Alghe, conservan-

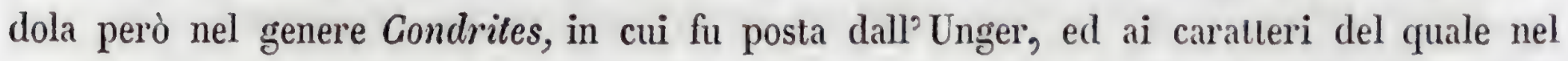
vero maggiormente $s^{2}$ attaglia.

\section{Chondrites Iumbricarius Münst.}

Gh. frondibus aggregatis implexis, dichotome ramosis, ramis cylindraceis in ectypis compressis, apicibus attenuatis.

Chondrites lumbricarius Münst. Beitr. zur Petref.-kund. Fase. VI. pag. 79, tab. 2. fig. 1. - Uvg. Conspect. Flor. primord. in Ghlor. Prolog. pag. xxvir.; Gen. et Spec. plant. foss. pag. 19. - Brons Ind. Palaeont. P. I. pag. 294. - Gieber Deutschl. Petrefact. pag. 10, n. 79.

In calcareo scissili ad Solenhofen.

L' esemplare, di cui il Münster ci porge la figura, apparisce composto da un ammasso di frondi insieme aggruppate, le quali cosi riunite occupano uno spazio circolare, il cui diametro raggiunge i ventisei centimetri. Nella parte centrale si scorgono maggiormente avviluppate fra loro, e formano una specie di plesso rilevato, che verso gli orli si dirada, mentre tulto all' intorno le ramificazioni si vanno gradatamente svolgendo ed isolando. Le frondi si ramificano per dicotomia, ed il loro diametro, come pure quello dei rami, è di circa un millimetro. Questi ultimi sono pure spesso dicotomi, e si assottigliano verso le estremità.

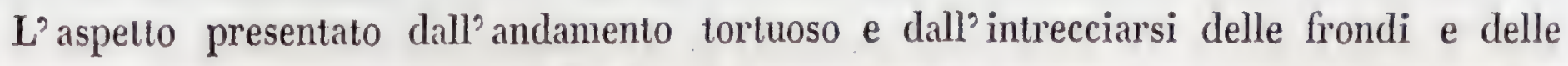
ramificazioni, rammenta quello delle Lombricarie, tanto frequenti nel calcare litografico di Solenhofen.

\section{Chondrites elongatus Presl.}

Gh. fronde stipitata? ramosa, ramis longissimis arcuatis, apicem rersus parumper angustatis, ramulis oppositis divergentibus angustato-acuminatis.

Chondrites elongatus Presi in Sterna. Vers. Flor. Vorw. II. pag. 104, tab. 28. fig. 2. Endu. Gen. plant. Suppl. III. pag. 55. - UNG. Gonspect. Flor. primord. in Ghlor. Protog. pag. xxvir.; Gen. et Spec. plant. foss. pag. 19. - Brong. Ind. Palaeont. P. I. pag. 294. Gieber Deutschl. Petrefact. pag. 10, n. 78 .

Caulerpites elongatus Münst. in litt. ad SтвRкв. 
In calcareo scissili formationis jurassicae ad Eichstedt.

Questa specie, la quale giunge colle sue ramificazioni ad una lunghezza di circa quaranta centimetri, ha una fronda di color bruno, che nella base, per un tratto di tre centimetri e mezzo, si mantiene larga tredici millimetri. Indi si pârte in più rami, che si prolungano curvandosi irregolarmente, ed hanno un diametro variante dai due ai quattro millimetri. I principali diminuiscono gradatamente di larghezza, e s'assottigliano verso le estremità, che sono sempre ottuse, ed in alcuni divise in due espansioni rotondate d'ineguale lunghezza. Dai lati dei rami sorgono rarissimi dei ramoscelli opposti patenti ed acuminati.

I signori Unger e Giebel annoverano quest ${ }^{2} A \lg a$ fra le specie proprie dell' 0 Oolite. Bronn all' incontro nel suo Enumerator Palaentologicus, in cui la parte che risguarda le piante fossili fu elaborata dal Göppert, la colloca nel Lias. È però indubitato che la calcarea scissile di Eichsted, al pari di quella di Solenhofen, appartenga al Mittler Weis-

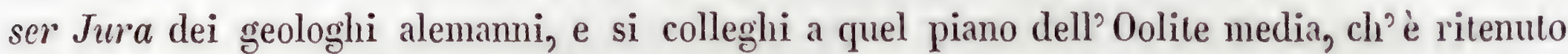
corrispondere alla parte inferiore del Coral rag dei geologhi inglesi.

\section{Chondrites Targionii Sternb.}

Tav, I. fig. 4.

Gh. fronde pinnatim vel bipinnatim ramosa, ramis filiformibus inaequalibus aequilatis, integris vel rarius furcatis obtusis.

Chondrites Targionii Sтекхв. Vers. Flor. Vorw. II. pag. 25, tab. 9. fig. 5-4. - Monris Gat. Brit. foss. pag. 5. - Expr. Gen. plant. Suppl. III. pag. 5วั. - UNG. Synops. plant. foss. pag. 9.; Conspect. Flor. primord. in Chlor. Prolog. pag. xxvir.; Gen. et Spec. plant. foss. pag. 16. - Brons Ind. Palaeont. P. I. pag. 294.; Leth. geogn. edit. 3. fase. 4. pag. 45 , fase. 5̋. pag. 108 , tab. 28. fig. 3. - Ввокт. Tabl. gen. végét. foss. pag. 9. - Sснағнаürt Sudbayr. Alpen, pag. 159 , tab. 3. fig. 2. - Gieber Deutschl. Petrefact. pag. 11, n. ${ }^{\circ} 86$.

Fucoides (Gigartinites) Targionii Brosg. Hist. végét. foss. I. pag. 56,57 et 84, tab. 4. fig. 2-6.; Prodr. pag. 20 et 204.

Fucoides Targionii MAnt. Geol. of the S. E. of England pag. 163. - Zeuschrer in Leowiard et Brown Neu. Jahr. 1846, pag. 178, 180 et 182. - Puscr. Polen. Palaeont. pag. 3 , tab. 1. fig. 1. - Prera Descriz. Terr. Etrur. pag. 19, tab. 2. fig. 1. - Mrrar. Struct. Alp. Apenn. and Carpath., in Quart. Journ. Geol. Soc. Lond. 1848, pag. 278.

Fucoides (Chondrites) Targionii Menegh. e SAvi Gonsiderazioni sulla Geologia stratigrafica della Toscana, P. III. pag. 381. 
In calcareo margaceo schistis versicoloribus interjecto formationis jurassicae inferioris Etruriae (Meneghini et Savi). In formatione jurassica ad M. Tatra (Zeulschner). Nec non in formationibus cretaceis et tertiariis Italiae, Germaniae, Helvetiae et Angliae.

$L^{\prime}$ esemplare, di cui porgo la figura, ha la fronda gracile, angusta, bipinnata, $0^{9}$ rami filiformi, indivisi o forcati, oltusi, spesso allungati, distanti fra loro e d $d^{3}$ ineguale lunghezza.

Pel modo di ramificazione si avvicina al Ch. Targionii var. $\gamma$ confertus dello Sternberg (BRosG. Hist. végét. foss. I. tab. 4. figg. 4. 5 ). Si distingue però da questa varietà per avere un aspelto più gracile, ed i rami molto più rari e sparsi, anzichè addensati.

Parlarono i primi di quest'Alga il Bartolini (Act. Acad. Sien. 1770, Vol. VIII. pag. 204, cum Iconibus), e Giovanni Targioni-Tozzetli (Relazione di alcuni Viaggi nella Toscana, ediz. seconda, 1787, Tom. X. pag. 81). Poscia il Brongniart la descrisse e figurò nelle varie forme solto cui si presenta, avendola studiata sopra esemplari provenienti dall' Inghilterra, dalla Svizzera, dalla Toscana, e particolarmente sopra quelli esistenti nella collezione stessa del Targioni. Lo Sternberg, ponendola nel genere Chondrites, ne descrisse cinque varietà, che distinse $c^{5}$ nomi di Ch. Targionii $\alpha$ fastigiatus; $\beta$ divaricalus; $\gamma$ confertus; $\delta$ expansus; $\varepsilon$ flexuosus: e di ciascheduna diede la figura. Ho cercato invano se taluna di queste forme potesse considerarsi propria di una formazione piuttosto che di un'altra. Per molto tempo si credette propria soltanto dei terreni cretacei superiori, e specialmente del piano della creta clorilica; da ciò ne veme che il macigno toscano, in cui abbonda, fu per più anni creduto appartenere all'epoca secondaria, fino a che gli studj paleontologici e stratigrafici instituiti dal Murchison posero in chiara luce la sua spetlanza all'epoca terziaria.

Lo Zeuschner nel 1846 trovava il Chondrites Targionii sul gruppo del Tatra nei Carpazj in una roccia con fossili giurassici; e recentemente i Professori Meneghini e Savi scoprivano questa specie a Pizzo d ${ }^{2}$ Uccello nelle Alpi Apuane e al Monte di Repole nei monti Pisani, nel calcare marnoso interposto agli scisti varicolori della Toscana, i quali pe' caralteri paleontologici e stratigrafici sembrano ivi costituire il piano inferiore dei depositi oolitici. Per tale motivo ho annoverato il Chondrites Targionii fra le piante dell' 0 olite, ed ho quì riportata la figura dell' esemplare trovato dai geologhi testè nominati, e che mi fu gentilmente comunicata dal Prof. Meneghini.

Il Chondrites Bollensis del Kurr, osservato nel Lias del Würtemberg, mostra tanta analogia con questa specie da polernelo considerare quasi semplice varietà. Da tutto ciò consegue, che il Chondrites Turgionii non può essere adoperato per la identificazione del terreno in cui se ne trovasse l'impronta. 


\section{Chondrites furcatus Sternb.}

Tav. II. fig. 1 .

Ch. fronde subdichotoma vel irregulariter bipinnata, ramis patentibus suboppositis, ramulis sparsis elongato-clavatis vel rectiusculis obtusis.

Chondriles furcalus Sterne. Vers. Flor. Vorw. II. p. 27. - Ende. Gen. plant. Suppl. III. pag. รั5. - Ung. Gonspect. Flor. primord. in Chlor. Protog. pag. xxvir.; Gen. et Spec. plant. foss. pag. 18. - Bronn Ind. Palaeont. P. I. pag. 294 (pro parte). - Brong. Tabl. gen. végét. foss. pag. 112. - GIeber Deutschl. Petrefuct. pag. 10, n. ${ }^{\circ} 83$.

Fucoides furcatus Brong. Mém. Soc. d'Hist. Nat. de Paris, Tom. I. pag. 509 , tab. 19. fig. 3. (excl. var. $\beta$ ). - Razumorsix Obs. min. sur les env. de Vienne, pag. $23_{2}$ tab. 4. fig. 25 . et 26.

Fucoides (Gigartinites) furcalus Brong. Hist. végét. foss. I. pag. 62, tab. 5. fig. 1. (excl. var. B); Prodrom. pag. 20, n. $^{\circ} 15$, et pag. 204, n. $^{\circ} 9$.

Fueoides (Chondrites) furcatus MrNegr. et $\mathrm{S}_{\mathrm{Avr}}$ Consid. sulla Geolog. stratigraf. della Toscana, pag. 381, n. ${ }^{\circ}$, et pag. 457, n. 129.

In calcareo margaceo schistis versicoloribus interjecto formationis jurassicae inferioris Etruriae in M. di Repole et in montibus ultra Serchio (Meneghini et Savi). Nec non in formationibus cretaceis et tertiariis.

Il chiarissimo Prof. G. Meneghini riferisce a questa specie il fucoide, di cui presento quì la figura, perchè, sebbene sia conosciuto, entra però nel novero di quelle specie che si mostrano promiscue a più formazioni, oltre l'oolitica.

Gli esemplari trovati dal suddetto naturalista in quel gruppo di strati, che per la posizione e pe' fossili può considerarsi rappresentare in Toscana il piano più basso del1: Oolite inferiore, hamno una fronda irregolarmente dicotomo-bipinnata, ed alqquanto più stretta della specie figurata dal Brongniart, raggiungendo essa appena nelle porzioni piu larghe i due millimetri. Le ramificazioni vi si scorgono divaricate, ma non ricurve; chè anzi i rami e ramoscelli sono costantemente dritti.

Tutte le estremità dell' esemplare figurato sono tronche, nè si può conoscere se ve ne fosse alcuna che presentasse il carattere dell' ingrossamento terminale clavato-allungato proprio della specie Brongniartiana.

Questa specie non deve confondersi col Chondrites furcatus di Presl (Stersib. loc. cit. pag. 103), che, come vedemmo più sopra, è sinonimo della Solenites? furcata di Lindley ed IIutlon, ossia Chondrites Solenites di Unger; nè manco col Fucoides furcatus figurato 


\section{$-31-$}

dal Pilla nella sua Descrizione del terreno Etrurio, inserita nel 1846 nei fascicoli di Maggio e Giugno del Giornale Il Gimento, ove colla fig. 3. della sua tavola 2. ci dà semplicemente la copia della fig. 2. tavola 3. della Histoire des végétaux fossiles del Brongniart. Ma questa figura rappresenta la varietà $\beta$ del Fucoides furcatus dello stesso Brongniart, la quale non si trovò mai in Toscana, ma bensì nel terreno oolitico di Stonesfield nell' Inghilterra, e che vedemmo ritenuta per ispecie distinta dallo Sternberg e da altri solto il nome di Halymenites ramulosus.

\section{Chondrites intricatus Sternb.}

Tav. II. fig. 2.

Ch. fronde decomposito-pinnatim ramosa, ramis ramulisque patenlibus, filiformibus, rectis crebris.

Chondrites intricatus Sterna. Vers. Flor. Vorw. II. pag. 26, tab. 7. fig. 5. a. - ENat. Gen. plant. Suppl. III. pag. 5̌5. - U U. Gonspect. Flor. primord. in Chlor. Prolog. pag. xxvir.; Gen. et Spec. plant. foss. pag. 17. - Brown Ind. Palacont. P. I. pag. 294.; Leth. geogn. edit. 5. fasc. 5้. pag. 109. - Brong. Tabl. gen. végét. foss. pag. 112. - Schaffaürt Südbayer. Alp. pag. 159, tab. 3. fig. 1. - Greser Deutschl. Petrefact. pag. 10, n. ${ }^{\circ} 81$.

Fucoides intricatus Bноќ. Mém. Soc. d'Hist. Nat. Paris. I. pag. 311, tab. 19. fig. 8. Sedgw. and Murcr. Géolog. Trans. P. III. pag. 332. - Puscir Palacont. 3. tab. 1. fig. 2. a. - Prla Descriz. del Terr. Etrurio, 1846, pag. 19, tab. 2. fig. 2.

Fucoides (Gigartinites) intricatus Brovg. Hist. végét. foss. I. pag. 59 , tab. 5. fig. 6-8.; Prodrom. pag. 20, n. ${ }^{\circ}$ 9, et pag. 204, n. $^{\circ}$ S.

Fucoides (Chondrites) intricatus Menegr. e SAvi Consid. sulla Geologia stratigraf. della Toscana, pag. 582, n. $^{\circ} 4$, el pag. 457, n. $^{\circ} 128$.

In eadem formatione cum prioribus alle Molina in M. Pisano et in montibus ultra Serchio invenerunt $c l$. Meneghini et Savi. Aliisque in locis in formationibus cretaceis et tertiariis frequenter occurrit.

L'esemplare, di cui il Prof. Meneghini mi ha favorito la figura, ha la fronda che si sparpaglia all' intorno, ramificandosi in forma ora dicotoma, ora pinnata. I rami principali sono patenti, sparsi, per lo più forcuti nell' estremità, e cogli apici ottusi. I ramoscelli sono brevi, ritti, meno patenti, e terminano essi pure ottusamente.

Come si scorgerà di leggieri dalla figura, la fronda nel presente esemplare non è cosi esile, e le ramificazioni non sono cosi intricate, come generalmente sogliono essere nel Chondrites intricatus dello Sternberg. Però questa specie, cui fu riferito dal Prof. Meneghini, è la sola alla quale maggiormente si atlagli per gli altri caralteri. 
Nell'opera dello Sternberg è citata la figura 4. della tavola 6., e questa citazione venne di poi ripetuta da tutti gli autori che parlarono del Ghondrites intricatus. Era però agevole il riconoscere che quella figura si riferisce alla Münsteria Hoessii (S SERnв.), e che invece il Chondrites intricatus è delineato nella figura 3. della tavola 7. nella parte superiore di un saggio, su cui si osserva pure una porzione della Münsteria medesima:

\section{Gen. XH. Sprinarecocates Sternb.}

Frons subcoriacea plana, dichotoma vel pinnata, aut filiformis.

Sterna. Vers. Flor. Vorw. II. pag. 28 (pro parte). - End. Gen. plant. Suppl. III. pag. 55 (pro parte). - UnGen Conspect. Flor. primord. in Chlor. Protog. pag. xxvirI.; Gen. et Spec. plant. foss. pag. 24 (pro parte). - Brown Leth. geogn. edit. 3. fase. 2. pag. 41.

\section{Sphaerococcites ligulatus Kurr}

S. fronde dichotome vel irregulariter ramosa, filiformi, ramis pinnatis, pinnulis lanceolatis acuminatis.

Sphaerococcites ligulatus Kunn Beitr. zur foss. Flor. der Juraf. Würtemb. pag. 16, tab. 2. fig. 5. - BRonn Ind. Palaeont. P. I. pag. 1162. - UNG. Gen. et Spec. plant. foss. pag. $23 . .-$ Gieber Deutschl. Petrefact. pag. 15, n. ${ }^{\circ} 115$.

In calcareo jurassico superiore ad Gerhausen prope Ulmam, nec non ad Neuffen.

Questa pianticella, lunga quattro centimetri, si divide irregolarmente in rami filiformi pinnati, piuttosto erelti, colle pinnule per lo più alterne, lanceolato-lineari, aventi la larghezza di un millimetro, e che terminano in punta lievemente acuminata.

Si trova in quantità nella calcaria scissile giurese superiore a Gerhausen presso Ulma, come pure a Nauffen negli strati inferiori del Giura bianco (Weisser Jura) dei geologhi alemanni.

\section{Sphaerococeites areuatus Presl}

S. fronde dichotoma multiramea, ramis ramulisque linearibus, acutis aut angustato-acuminatis, integerrimis, extus falcato-curvatis, apparenter unicostatis.

Sphaerococcites arcuatus $\mathrm{P}_{\mathrm{RESL}}$ in STERxB. Vers. Flor. Vorw. II. pag. 104. - MonRIS Gat. Brit. foss. pag. 23. - Ekdr. Gen. plant. Suppl. III. pag. 55. - Ung. Conspect. Flor. 


\section{$-33$}

primord. in Chlor. Protog. pag. xxvir.; Gen. et Spec. plant. foss. pag. 26. - Bross, Ind. Palneont. P. I. pag. 1162.

Fucoides arcuatus, Lindz. et Hutr. Foss. Flor. of Great Brit. Vol. III. tab. 185.

\section{In formatione oolithica ad Gristhorpe Angliae.}

Si distingue quest ${ }^{\top} \mathrm{Alga}$, come ce lo indica il nome specifico, per $l^{2}$ arcuazione delle sue ramificazioni. La fronda ne è consistente, e sembra segnata da qualche stria longitudinale. Essa si divide per dicotomia in più rami arcuati divergenti, che ove non sono troncati mostrano di terminare in punta assai acuminata. Nell' unico esemplare esistente, e che si trova nella collezione del Prof. Phillips, le porzioni inferiori della fronda, di cui però non si scorge il principio, hanno un diametro di quattro millimetri, che diminuisce gradatamente nei rami.

I signori Lindley e Hutton, avendo osservato su varj punti della fronda una linea biancastra scorrere per alcun tratto sul mezzo, parallela agli orli, propendono a credere

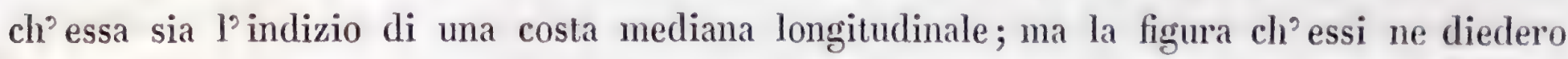
non esprime in modo abbastanza chiaro quest’apparenza.

Que ${ }^{2}$ due chiarissimi Naturalisti giustamente rimarcano che questa specie è diversa da tutte quelle che il Brongniart descrisse e figurò nella sua Histoire des végétaux fossiles. Presl scorgerebbe in essa qualche lontana affinità col suo Sphaerococcites crispiformis (Algacites crispiformis Schloth. nacht. Petref. I. pag. 45, tab. 4. fig. 1.), che il Brongniart non ammise fra le Alghe, sembrandogli piuttosto ch' esso presenti l'aspetto di una Rhizomorfa. A dir vero, alcnne porzioni dello Sphaerococcites crispiformis si ramificano in modo eguale a quello dei due rami visibili nella parte superiore dell' esemplare figurato nella Fossil Flora of great Britain; ma in tutto il resto queste due specie sono l'una dall'altra affatto distinte, come ne conviene lo stesso Presl.

\section{Sphaerococeites Meneghinii Zign.}

Tav. II. fig. 3.

S. fronde plana ramosa subdichotoma, ramis sparsis late linearibus aequilatis, infimis divaricatis arcuatis, superioribus erectis.

In calcareo argillaceo schistis versicoloribus interjecto formationis oolithicae inferioris Etruriae legerunt Professores Meneghini et Savi. 


\section{- 34}

La porzione visibile sull'esemplare qui figurato presenta una fronda piana eretta, avente un diametro costante di quattro millimetri, dalla cui parte inferiore si espande lateralmente un ramo semplice assai patente, che si prolunga fino all'orlo del saggio. Quattordici millimetri più in su, dallo stesso lato, sorge altro ramo molto ricurvo, lungo venti millimetri, e coll' estremità obbliquamente mozza. Subito dopo ne segue un terzo contiguo al precedente, ancor più arcuato, e vòlto allo stesso verso, che giunge fino al margine dell' esemplare. La fronda corre poscia ritta e senza rami per quindici millimetri, indi si pîte in due ramificazioni quasi eguali, di cui l'una dopo un tratto di quindici millimetri rimane mozza dal termine del saggio, l'altra finisce oltusamente un po prima di giungervi.

Questa specie, del cui disegno mi fu cortese il chiarissimo Prof. Cav. Giuseppe Meneghini, offre qualche punto di rassomiglianza collo Sphaerococcites affinis di Presl (redi Sternberg, Vers. Flor. der Vorwelt. II. pag. 28, tab. VII. fig. 1., figura che viene per errore citata dal Bromn nella sua Lethaea geognostica, Tom. II. pag. 45, a proposito della Cystoseiriles mutans dello Sternberg, che, come dimostrammo, non esiste) specialmente per l'arcuazione dei rami inferiori e per l'eguaglianza del loro diametro. Tuttavia stimai di poternela distinguere, perchè ha la fronda più eretta, e piuttosto dicotoma che bipimata, e le ramificazioni meno numerose, semplici, e la maggior parte più fra loro distanti.

\section{Sphacrococeites Savii Zign.}

Tav. II. fig. 4.

S. fronde erecta plana, inferne simplici superne tripartita, ramis ramulisque obliquis saepe longitudinaliter medio plicatis, marginibus leviter undulatis.

In schistis versicoloribus formationis oolithicae inferioris Etruriae legerunt Professores Meneghini et Savi.

La fronda, nell'esemplare di cui porgo la figura, corre ritta e senza rami per ventisei millimetri, mantenendosi uniforme nel diametro, chn è di cincue millimetri. Poscia si pârte in tre rami, due dei quali sorgono obbliquamente, opposti $l^{\prime}$ uno all'altro, ed hanno un diametro eguale a quello della parte inferiore della fronda, mentre il ramo mediano misura soli tre millimetri di larghezza, e dopo altrettanti di lunghezza rimane troncato. Da uno dei rami laterali si espande, dopo un tratto di circa quattordici millimetri, una seconda ramificazione, che si prolunga obbliquamente per ben tre centimetri, ed è un poco più stretta delle altre. Non è possibile conoscere come abbiano termine le estremità, essendochè negli esemplari è affatto mozza la parte superiore dei rami. I margini tanto 


\section{$-3 \breve{-}-$}

della fronda che delle ramificazioni si mostrano leggermente ondulati, e sui frammenti sparsi quà e là sulla superficie della roccia si distingue una specie di solco o piegatura, che ne segna longitudinalmente la parte mediana.

Anche questa specie, come la precedente, fu trovata in Toscana dai Professori Meneghini e Savi negli schisti che fanno parte del gruppo da essi riferito all'Oolite inferiore; e mi avvertiva il primo, che su altri esemplari rinvenuti da poi aveva potuto riconoscere quasi costante il carattere della piegatura longitudinale, che soltanto sopra alcuni frammenti del nostro si osserva.

\section{Sphaerococeites? dichotomus Zign.}

S. fronde compressa? dichotoma; ramis inaequalibus patulis obtuse rotundatis.

Fucoides dichotomus. Monns in Capt. Grant Mem. to illustr. a Geol. Map of Cutch. Transact. of the Gcol. Soc. of London. Series II. Vol. V. Pars II. pag. 289, tab. 21. fig. 7.

In arenaria et in argilla carbonifera formationis oolithicae ad Gutch Indiae.

Riferisco con qualche dubio al genere Sphaerococcites l’impronta trovata dal capitano Grant con altri resti vegetali nell'argilla schistosa e nell'arenaria che compongono la formazione carbonifera del paese di Cutch nelle Indie, adeguata agli strati carboniferi dell'Oolite d'Inghilterra. Il sig. Morris, che illustrò le piante figurate nella Memoria del capitano Grant, appose a quest’Alga la frase specifica che qui riporto, e la distinse col nome di Fucoides dichotomus. La figura non permette che vi si riscontrino altri caratteri, all' infuori di alcune ineguaglianze nel contorno, dipendenti forse dal callivo stato di conservazione dell' esemplare.

Gen. AHI. Hewommevites Trevis.

Frons plana, solida, ecostata, cartilaginea, flabellatim expansa segmentis dichotomis. Sporangia frondi immersa creberrima.

Trevis. 1849 in mss. Edit. II. Nomencl. Algarum.

\section{Hhodymenites ciliatus Trevis.}

Rh. fronde plana ramosa supra basim flabellato-digitata, ramis dichotomis erectis late linearibus, superne constrictis, obtusis, marginibus sinuato-ciliatodenlatis, creberrime punctata. 


\section{$-36-$}

Rhodymenites ciliatus. Trevis. 1849 in mss. Edit. II. Nomencl. Algarum.

Sphaerococcites ciliatus. Strenns. Vers. Flor. Vorw. II. pag. 28, tab. 4. fig. 1. - Endich. Gen. plant. Suppl. III. pag. 55, n. ${ }^{\circ}$ 1. - Uvg. Synops. pag. 15; Conspect. Flor. primord. in Chlor. Protog. pag. xxvir.; Gen. et Spec. plant. foss. pag. 24. - Brons, Ind. Pulaeont. P. I. pag. 1162. - Brong. Tabl. Gen. végét. foss. pag. 103. - Greber, Deutschl. Petref. pag. 13, n。114.

In calcareo lithographico ad Solenhofen.

La base della fronda, ristrelta in principio a sei millimetri di larghezza, si espande poscia fino a raggiungere un diametro di due centimetri per ogni verso. Da questo punto si estendono in forma digitata cinque rami, tre dei quali più lunghi ed inegualmente dicolomi, due molto più brevi e semplici. Queste ramificazioni hanno alla base un diametro di cinque in sei millimetri, che varia superiormente, e giunge fino ai dodici; indi gradatamente diminuisce verso le estremità. Queste ultime non terminano tutte in egual modo: alcune essendo sinuato-ciliato-dentate, come lo è in ogni parte il margine di tutta la fronda; altre prolungandosi in una espansione claviforme più strelta del ramo, e cogli orli affatto liscj.

Questa specie, che nell' esemplare dello Sternberg arriva ad una lunghezza di oltre a tredici centimetri, è spessamente punteggiata in ogni sua parte.

Il Cavalliere Vittore Trevisan proponeva sino dal 1849 questo genere nei manoscritti da lui predisposti per una compiuta nuova edizione del suo Nomenclator Algarum, ravvisando nello Sphaerococcites ciliatus dello Sternberg caratteri sufficienti per separarlo dal genere cui lo aveva riferito l'Autore della Flora der Worwelt. Notava bensi questo ultimo la grande rassomiglianza di questa specie collo Sphaerococcus laciniatus di Lynbye, ch'è ora la Rhodymenia laciniata di Greville, Alg. Brit. pag. 86, ed è per ciò che il 'Trevisan basava su quest'analogia i caratteri del nuovo genere Rhodymenites.

\section{Gen. Xy. Granuraria Pomel.}

Frons ramosa cylindrica vel plana, coriacea, crassa, dichotoma vel pinnata mamillis granuliformibus irregularibus creberrimis obtecta, saepe sulcis tenuissimis transversim reticulata. Rami, ramulisque dichotome vel pinnatim ramosi.

Granularia Ponel. Materiaux pour sersir à la Flor. Jurass. de la France, in Amtl. Bericht über die Versamml. der Gesellschaf. Deutsch. Naturforsch. in Aachen. September 1847. Aachen 1849, pag. $\mathbf{3 5 3}$ (exclus. pro parte synon.).

Phymatoderma. Broxg. Tabl. Gen. végét. foss. pag. 10. Paris 1849. 


\section{Granularia Iinearis Zign.}

Tav. II. fig. $\breve{.}$.

G. frondibus compressis, linearibus, dichotomis et lateraliter ramosis ramis obliquis vel recurvis divaricatis; papillis granuliformibus minutis.

Fucoides (Phymatoderma) linearis MEnegri. Consid. sulla Geol. stratigraf. della Toscana, in Append. ad version. ital. Dissert. clarissimi Eq. R. I. Murchison, De struct. geol. Alp., Apenn. et Carpath., pag. 582, n. ${ }^{\circ} 5$; pag. 390, n. ${ }^{\circ}$; et pag. 457, n. ${ }^{\circ} 130$.

In schistis versicoloribus in loco dicto le Molina ad M. Pisano simul cum Chond. intricato, nec non inter Campiglia et Montieri.

Sull' esemplare, di cui il Prof. Meneghini mi spedi la figura, si veggono sei porzioni di questo Fucoide, dalle quali non possiamo rilevare qual fosse la lunghezza della fronda, ed il modo con cui avea termine, perchè giungono quasi tutte all'orlo del saggio, o sono ricoperte dalla roccia in guisa da impedire che se ne possano scorgere le estremita. 'Tuttavia il detto Professore, che ne esaminò più esemplari, propende a ritenere che la lunghezza totale di quest ${ }^{2}$ Alga si prolungasse oltre $\mathrm{i}$ due decimetri.

Il diametro dei rami principali giunge ai dodici millimetri, e quello dei secondarj si limita a quattro. Le dicotomie si partono obliquamente ad angolo acuto, ed i rami sono superiormente divaricati, ora diritti, ora lievemente ricurvi.

Le papille minutissime ed irregolari si osservano rappresentate nel frammento ingrandito; debbo però avvertire che il disegnatore le ha alcun poco esagerate.

\section{Granularia repanda Pomel.}

G. fronde ramosissima; ramis ramulisque aequalibus repandis, gracilibus; ramusculis obtusiusculis brevibus vel elongatis, granulis minutis.

Granularia repanda. Pomer, Materiaux pour servir à la Flor. foss. des terr. jurass. de la France, in Amtl. Berich. über die Versamml. der Gesellseh. Deutsch. Naturforscher, in Aachen, Septemb.1847. Aachen 1849, pag. 354 .

In formatione oolithica media ad Chateauroux Galliae.

Non trovo caratteri sufficienti per separare il genere Phymatoderma, stabilito dal Brongniart nel suo Tableau des Genres des végétaux fossiles, stampato nel 1849, dal genere Granularia, fondato da Pomel nella sua Memoria intitolata Materiaux pour 


\section{$-38$}

servir à la Flore fossile des terrains jurassiques de la France, letta alla Rimione dei Medici e Naturalisti Alemanni, ch'ebbe luogo in Aachen nel Settembre 1847, ma che vide la luce soltanto nell' amo 1849, quando si publicarono gli Atti di quell’ Adunanza.

La specie che servì al Pomel per creare il genere Granularia è quella stessa, su cui s'appoggia il Brongniart per istabilire il genere Phymatoderma, l’uno e l'altro citando quale specie tipica del proprio genere l'Algacites granulatus di Schlotheim, o Sphaerococcites crenulatus di Sternberg.

E forza quindi ritenere il solo genere Gramularia, che ha una decisa priorità su quello del Brongniart, perchè reso noto in una publica adunanza di Naturalisti due anni prima. Però sostituendolo al genere Phymatoderma non intendo di abbracciare con esso tutte le specie poste dal Pomel fra le Granularie, e mi limito a comprendervi soltanto quelle Alghe, i cui caratteri si avvicinano maggiormente a quelli della specie indicata qual tipo del genere, fra le quali mi sembra che le due testè descritte possano senza esitanza essere annoverate.

\section{Gen. Xv. Coralenvites Ung.}

\section{Frons rigida calcarea arliculato ramosa.}

Uva. Chlor. Protogea, pag. 127.; Gen. et Spec, plant. foss. pag, 24. - Brosg. Tabl. Gen. végét. foss. pag. 9. - Massar, Piante fossili vicentine, pag. 71.; Monograf. Gen. Corallinites in Stud. Paleont. Pars VI. pag. 56 - 59.

\section{Corallinites arbuseula Ung.}

G. fronde plana filiformi ramosa, ramis pinnatis, ramulis gracilibus apice incrassatis obtusis articulis obsoletis.

Corallinites arbuscula. Uxa. Chlor. Protog. pag. 127, tab. 59. fig. 6.; Gen. et Spec. plant. foss. pag. 24. - Brong. Tabl. Gen. pégét. foss. pag. 105. - Gieber, Deutchl. pelref. pag. 12, n. 106. - Massat. Monograf. Gen. Corallin. in Op. cit., pag. 59.

In calcareo formationis oolithicae ad Pechgraben prope Weiher Austriae inferioris.

Questa pianticella a fronda compressa filiforme, ramificata in forma irregolarmente bi-tripinnata, ha i ramoscelli ristretti, alquanto rigonfj ed ottusi nelle estremità. Non si scorgono in essa distintamente segnate le articolazioni; contuttociò nel complesso si avvicina $d^{\prime}$ assai alle Coralline viventi. Nell' esemplare figurato dall' Unger la lunghezza della 
fronda oltrepassa di poco i quattro centimetri, e tanto i rami che i ramoscelli hanno un diametro che nei punti ingrossati, cioè verso le estremità, non arriva ad un millimetro.

Fu trovata presso Weilıer nell'Austria inferiore in una calcarea grigia che certo appartiene, secondo l’Unger, alla serie oolitica.

\section{Corallinites Halimeda Ung.}

G. fronde crassiuscula articulata dichotome ramosa, ramis divaricatis, articulis distinctis, ellipticis vel orbicularibus subcompressis.

Corallinites Halimeda. Uvg. Chlor. Protog. pag. 127, tab. 59. fig. 7.; Gen. ct Spec. plant. foss. pag. 24. - Brovg. Tabl. Ger. végét. foss. pag. 105. — Gieber, Deutschl. petref. pag. 12, n。 107. - Massal. Monograf. Gen. Corallin. in Op. cit., pag. 59.

In calcareo jurassico (Weisser jura) ad rivum Reich-Raming Austriae inferioris.

La fronda ramificata per dicotomia, $\operatorname{co}^{2}$ rami pure dicotomi e divergenti, ha da per tutto un diametro di tre in quattro millimetri, ed è composta di articolazioni rotondate ora ellittiche, ora orbicolari, neltamente marcate.

L’impressione lasciata sulla roccia dà a divedere che gli articoli fossero alcpuanto rigidi e consistenti, e piultosto compressi che globulari.

Il chiarissimo Prof. A. Massalongo nella sua Monografia del genere Corallinites osserva giustamente che questa specie ha maggiori analogie col genere Halimeda, che col genere Corallina.

\section{Classis III. CALAMARIAE.}

Nell'ordinamento dei diversi generi componenti questa Classe, e riferibili a specie proprie dell' 0 olite, io mi sono attenuto alle riforme introdotte recentemente dal chiarissimo Prof. Costantino di Ettingshausen. Le ragioni da lui addolte per riunire i generi Asterophyllites e Volkmamia al genere Calamites mi sembrarono atte a convincere anche i più avversi a codesto genere di rimioni, perchè fondate su diligenti indagini, instituite sopra un ragguardevole numero di esemplari benissimo conservati (1) Da ciò

(1) Vedi le Memorie dell'Ettingshausen, intitolate: Beitrëge zur Flora der Vorwelt. I. Untersuchungen über mehrere Calamiten-und Asterophylliten-Formen; II, Monographia Calamariarum fossilium; 
deriva che la Classe delle Calamarie, anzichè dividersi nei tre Ordini delle Calanitee, delle Asterofillitee e delle Equisetacee, deve quindi innanzi, secondo l'Euingshausen, pârtirsi ne? due soli Ordini delle Calamitee e delle Equisetacee; nel primo dei quali si comprenderanno i generi Galamites, Calamitea, Huttonia, Annularia, Sphenophyllum, Hippurites, Phyllotheca, Bornia, Boclischia, Anarthrocanna (1); e nel secondo i generi Equisetites e Columnaria.

Nell'ordine cronologico delle formazioni sedimentarie veggiamo comparire prime le Calamiti, le Bornie e le Anartrocanne, che si rinvengono nei terreni di transizione. Nella formazione carbonifera spariscono le Bornie, le Anartrocanne, e rimangono le Calamiti, accompagnate da altri generi che ivi si mostrano per la prima volta. A questi appartengono le Huttonie, le Annularie, gli Sfenofilli, le Ippuriti e le Bockschie, a cui si aggiungono in questo terreno le Equisetiti e le Columnarie. La maggior parte di questi generi non si ripete nelle successive formazioni. Nell'arenaria rossa i due Ordini sono rappresentati dai generi Calamitea ed Equisetites, e le sole Calamili, accompagnate dalle Equisetiti, si fanno nuovamente vedere nelle stratificazioni dell’arenaria variegata e del Keuper.

Nel lias si rinvennero finora soltanto le Equisetiti, che tornano a comparire nell'00lite unitamente al nuovo genere Phyllotheca, e ad alcune forme che per ora sono collocale nei generi Galamites e Sphaenophyllum, a cui maggiormente si approssimano, finchè migliori esemplari ne chiariscano la vera natura.

Nella formazione Wealdiana e nei terreni terziarj il genere Equisetites rimane solo rappresentante di questa Classe.

In quanto alle Calamarie proprie della Flora dell'0olite, non sono molti anni ch's esse limitavansi a due sole specie di Equisetacee: l'Equisetum columnare del Brongniart e 1'Equisetum laterale di Phillips. A queste si aggiunsero di poi il Calamites Hoerensis, scoperto dal Prof. Hisinger in questa formazione presso Hoer nella Scania (2); ed il Calamites Lehmanniamus, trovato dal Göppert nel terreno giurese di Ludwigsdorf nella

nelle Naturwissenschaftlichen Abhandlungen del Cav. di Haidinger, stampate in Vienna nel 18531; e la Steinkohlenflora von Radnitz in Bölmeir, inserita nelle Memorie dell' I. R. Istituto Geologico dell' Impero per l'anno 1833.

(1) Nel Vol. XI. delle Memorie dell'I. R. Academia delle Scienze di Vienna, stampate nel 18ä6, il chiarissimo Prof. Unger inseri un lavoro molto importante sulla Flora degli schisti e delle arenarie della Turingia, nel quale propone una nuova classificazione delle Calamarie, basata sull'esame microscopico dell'interna loro struttura, dalla quale desume i caratteri per fondare i nuovi generi Haplocalamus, Kalymna, Calamopteris, Calamosyrinx, Calamopithys. In quanto poi all'Asterophyllites coronatus, che vi è figurato e descritto, esso presenta, a parer mio, le più grandi analogíe col genere Phyllotheca del Brongniart.

(2) Hisinger, Lethaea suecica, Suppl. II. (1840), pag. 5ै, tab. 38. fig. 8. 


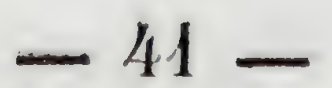

Slesia prussiana (1). Poco dopo il sig. Bean raccoglieva in Inghilterra nei terreni oolitici di Scarborough un esemplare che, illustrato dal sig. Bunbury, ed intitolato al ritrovatore sotto il nome di Calamites Beani, venne ad arricchire di un'altra specie la Flora di cui ci occupiamo (2). Finalmente il Prof. Rogers scopriva in America due nuove forme riferibili a questo genere nel terreno carbonifero della Virginia orientale, che buone ragioni inducono a ritenere spettante alla parte inferiore dell'Oolite (3). Anche l'Equisetum laterale di Phillips, dopo che il Bunbury ebbe ad osservarne un esemplare munito di foglie verticillate, per cui gli si era desta l'idea che dovesse annoverarsi fra le Asterofilliti, viene per ciò stesso ad aumentare il novero delle Calamiti della formazione oolitica.

Nei terreni carboniferi delle Indie e dell'Australia, che, come ho dimostrato nella mia Memoria sulla Flora fossile dell'Oolite, inserita nel Vol. VI. delle Memorie dell’ I. R. Istituto Veneto, deggiono, per le osservazioni del Greenough, del Bumbury e del II' Coy, considerarsi come appartenenti all'Oolite inferiore, i generi Vertebraria, Trizygia e Plyllotheca aggiungono altre sei specie alle Calamarie di questo terreno, e ci presentano delle forme che rammentano gli Sfenofilli e le Ippuriti delle formazioni più antiche. Alcuna di queste forme si ripete nell'Oolite delle Alpi venete, ove sopra buon numero di esemplari potei riconoscere l'esistenza di due nuove specie di Phyllotheca.

In quanto poi alle Equisetacee, esse sono rappresentate in questa parte della formazione giurese da quattro sole specie bene determinate: l'Equisetites colummaris STERna., dei depositi oolitici della Scozia, dell' Inghilterra e dell'America; l' Equisetites arundiniformis di Rogers, trovato coll'antecedente nel terreno carbonifero della Virginia orientale; ed altre due specie bene distinte, rinvenute negli strati calcarei sottoposti al terreno Oxfordiano nel Veronese.

Per questi ritrovamenti le Calamarie sommano ora nell'Oolite a diciotto, cioè quattordici Calamilee e quattro Equisetacee. Per ciò che risguarda poi la riunione di queste diverse forme nella Classe delle Calamarie, e la loro distribuzione nei generi Galamites, Sphenophyllum, Phyllotheca, Equisetites, molto vi sarebbe a dire, se ne togli il genere Equisetites, le cui specie ci presentano la maggior parte dei caralteri che distinguono gli Equiseti viventi; ed è soltanto perchè nelle specie oolitiche non mi sono ancora imbattuto nella fruttificazione, che stimai miglior consiglio serbar loro la denominazione generica di Equisetites, anzichè porle a dirittura fra gli Equisetum.

(1) Göppert. Ueber die fossile Flora der mittleren Jura-schichten in Ober-schlesien nell' Uebersicht der Arbeiten und Verïnderungen der schlesischen Gesellschaft, etc. Breslau 1846.

(2) Bumbury. On some Fossil Plants from the Jurassic strata of the Yorkshire Coast Quart. Journ. Geol. Soc. Lond. 18ä1. Vol. VII. pag. 179.

(3) Rogers. On the Age of the Coal Rocks of Eastern Virginia, Reports of the Assoc. of Americ. Geol. and Naturalists. Boston 1843, pag. 298. 
Le piante dell' Oolite comprese nel genere Calamites ci porgono pochi caratteri veramente decisi, per cui si devono calcolare poste provisoriamente fra le Calamiti fino a che nuove indagini ci dieno lumi maggiori. A dir vero, è molto dubia la natura anche di tutte le altre specie di questo.genere, che si rinvennero assai più numerose e meglio conservate nei terreni più antichi.

I generi Vertebraria e Trizygia di Forbes Royle (1) non mi sembrarono munili di caratteri sufficiènti a distinguerli dagli Sfenofilli; perciò, uniformandomi alle osservazioni del Brongniart ed al parere dell' Unger e dell' Ettingshausen, ne annoverai le specie nel genere Sphenophyllum.

È degna di rimarco la presenza nei terreni oolitici del Veneto di una forma sinora trovata soltanto in quelli delle terre Australi e delle Indie, voglio dire del genere Phyllotheca, al quale io riferisco alcuni cauli articolati forniti di guaine, il cui lembo si risolve in appendici fogliacee, che si osservano di frequente negli strati fitolitiferi della nostra formazione oolitica. Questi resti simigliano di molto all'Asterophyllites coronatus dell' Unger; pianta che, stando alle fiğure ch'egli ne diede, io collocherei piuttosto tra le Filloteche.

Assai disparate sono le opinioni intorno alle classificazioni delle varie forme che comprendiamo nei due generi Sphenophyllum e Phyllotheca. M'Coy (2) riferisce le Vertebrarie di Forbes Royle alle Marsileacee, e le Filloteche alle Casuarinee. Brongniart (3), unendo le Trizigie agli Sfenofilli, pone questi e le Filloteche fra le sue Asterofillitee nelle Dicotiledoni ginnosperme. Unger (4) le colloca fra le Calamarie nell' Ordine delle Asterofillitee; ed Ettingshausen (ă) avendo riunito quest' Ordine a quello delle Calamitee; le annovera fra queste ultime. $\mathrm{Ed}$ in vero è forza ammettere che le piante comprese nel genere Sphenophyllum formano un gruppo che offre ben poche analogie cogli altri di cui è composta questa Classe. La forma delle foglioline che ne compongono i verticilli le distingue netlamente dagli altri generi, e la loro collocazione fra le Calamarie verrebbe solo giustificata dal fatto, che la loro fruttificazione simiglia di molto a quella delle Asterofilliti, che, come dicemmo, sono ora riunile dall' Ettingshausen al genere Galamites. Tuttavia i loro organi della fruttificazione non si trovarono finora cosi chiaramente espressi

(1) Forbes Royle. Illustrations of the Botany and other branches of the Natural History of the Himalayan Mounlains and of the Flora of Cashmere. Vol. Il. plate 2. fossil plants of the Burdwan coal formation.

(2) M'Coy. On the Fossil Botany and Zoology of the of Rocks associated with the Coal in Australia. Annals and Magaz. of Nat. Hist. London 1847, Vol. XX. pag. 145.

(3) Brongniart. Tableau des Genres des végétaux fossiles. Paris 1849.

(4) Unger. Genera et Species plantarum fossilium. Vindobonae 1850, pag. 71-73.

(b) Ettingshausen. Monographia Calamariarum fossilium, pag. 23-24. 
da autorizzare che si proceda con sicurezza alla loro classificazione. Perciò mi trovai indotto a lasciare questo genere unitamente alle Filloteche in una Classe in cui sono comprese altre forme non meno problematiche, attendendo che future scoperte ci additino il posto che dovranno realmente occupare. $\mathrm{E}$ ciò tanto più, ch's essendo il presente lavoro circoscritto alle sole piante dell' Oolite, il numero delle Calamarie proprie dei terreni oolitici non è così rilevante da rendere necessaria una riforma di questa Classe: al che fare sarebbe d'uopo rivolgere i propri studj ai materiali ben più copiosi che ci porgono le formazioni delle epoche anteriori.

\section{Ordo VI. Calamiteae.}

\section{Gen. X. Calanites Suck.}

Caulis cylindricus, regulariter costatus, articulatus, sulcis articulorum alternantibus, saepius convergentibns. Vaginae patentes, profunde multidentatae rel earum loco in apicibus arliculorum folia, tuberculis infra articulationem inter sulcos symmetrice distributis, insita, caducissima. Rami mox in verticillis dispositi mox solitarii articulati, saepius striati, foliati. Folia verticillata, linearia, libera vel basi connata, saepius crebra. Fructificatio spicata. Spicae cylindricae, elongatae, verticillato foliatae. Sporocarpia in axillis foliorum, solitaria nuculaeformia.

Suckow in Act. Acad. Theod. Palat. V. 559. - Brosg. Prodr. pag. 57.; Hist. végét. foss. I. pag. 121. - Exde. Gen. plent. pag. 58. - Ung. Synops. pag. 20.; Gen. et Spec. plant. foss. pag. 44. - Extrvgsir. Beitr. zur Flor. d. Vorwelt II. Monographia Calamariarum, pag. 8.

\section{Calamites Cehmannianus Göpp.}

G. caule articulato, articulis subremotis tumescentibus, in statu juniori tuberculatis ad articulationes subconniventibus convexis striatis, sulcis planis tenuissime striatis.

Calamiles Lehmannianus. Görp. foss. l. d. mittl. Jura in Ucbers. d. Arbeit. und. Veränd. d. Schles. Gcsellsch. 1846, pag. 145, tab. I. fig. 1-3. - Uva. Synops. pag. 23; Gen. et Sprec. plant. foss. pag. 49. - Etrixgsi. Monograph. Calam. in Beitr. z. Flor. d. Vorvelt. pag. 15. - Giebel, Deulschl. Petref. pag. 25. 


\section{$-44-$}

In argilla scissili ferruminosa formationis oolithicae inferioris ad Wilmsdorf prope Landsberg Silesiae.

Il caule varia di grossezza a seconda dell' età, e cosi pure la lunghezza degl'internodj; ma si mantiene costante il carattere, che vale principalmente a distinguere questa specie dalle altre; cioè la presenza delle finissime strie longitudinali e parallele fra di loro, che segnano egualmente tanto i solchi, quanto le coste convesse da cui è percorsa.

Due esemplari, che si riferiscono ad individui d'età diversa, sono figurati e descritti dal chiarissimo Prof. Göppert nella sua Memoria sulla Flora fossile degli strati giurassici medj della Slesia superiore, inserita negli Aui della Società delle Scienze naturali della Slesia per l’anno 1845.

Nella figura 1., in cui si vede rappresentato lo stato giovane di questa specie, essa ha un diametro di sei millimetri, che s'aumenta di un millimetro al punto di congiunzione delle articolazioni, le quali sono discoste $l^{\text {'}}$ una dall' altra per tre centimetri e mezzo. Perciò è lecito ritenere che la lunghezza degli internodj equivalga a sei volte circa il diametro del caule. Alla fig. 2. si scorge una grossa porzione del caule, ormai giunto ad avere una larghezza di quasi sette centimetri, e clie mostra nella parte superiore le traccie di un'articolazione, sulla quale però non sono visibili i tubercoletti che sorgono in quel punto dalla estremità dei solchi ne' rami più giovani, come ci fa vedere la porzione ingrandita riportata dalla fig. 3. dello stesso Autore.

La formazione in cui il Göppert scoperse in Islesia lo strato fitolitifero che contiene questa specie unitamente ad altre, appartiene senz' alcun dubio all' epoca oolitica. I resti animali che si trovarono nelle roccie concomitanti presentano però una miscela di fossili spettanti parte al gruppo Oxfordiano e parte all' Oolite inferiore, sicchè non saprebbesi a quale dei due piani fosse da riferirsi lo strato che racchinde $\mathrm{i}$ resti vegetali, se questi ultimi non concorressero a togliere questa incertezza palesando, come vedremo più chiaramente in appresso, un'analogía ben marcata colla Flora che in altri paesi si osserva dominare nell' Oolite inferiore.

\section{Calamites Hoerensis Hising.}

C. caule cylindrico, articulis elongatis, 3-4 centim. longis, 3 cent. latis; costis circiter 1 millim. crassis, striaeformibus crebris.

Calamiles Hoerensis: Hisivg. Lelh. Suec. Suppl. II. (1840), pag. 5, tab. 38. fig, 8. - UNG. Synops. pag. 24.; Gen. et Spec. plant. foss. pag. 50. - Erriscsu. Monograph. Calam. in Beitr. z. Flor. d. Vorw. pag. 14. 
In arenaria ferruminosa ad Hoer Scaniae.

A prima giunta questa specie parrebbe di poco diversa dal Galamites arenaceus del Brongniart; e ciò fu eziandio rimarcato dal chiarissimo Autore della Lethaea Suecica, il quale a questo proposito cosi si esprime: "Calamite arenaceo Srerns. primo intuitu "similis, sed licet minor et angustior striis profundioribus et remotioribus exaratus. " A queste differenze aggiungeremo un altro carattere che si desume dalla diversa proporzione in cui sta la lunghezza degl'internodj rispetto al diametro del caule, giacchè mentre nel Calamites Hoerensis Hising. veggiamo la prima di poco eccedere la larghezza del caule, all' incontro nei varj stati del Calamites arenaceus, figurati e descritti finora, la lunghezza degl' internodj generalmente sorpassa di quattro e persino di sei volte il loro diametro trasversale.

Diverse sono le opinioni sull' epoca a cui si debba riferire $l^{\prime}$ arenaria fitolitifera di Hoer nella Scania. Brongniart, Hisinger e Braun propendono a collocarla nel Lias. Mantell scorge all ${ }^{p}$ incontro nei resti vegetali che contiene molta analogia con quelli dell’argilla di Weald. Nilsson e Forchhammer reputano invece questo deposito coevo a quello dell' Oolite di Scarborough nella Contea di York. Noi svolgeremo a suo luogo i motivi che c'indussero ad abbracciare quest'ultima opinione. Fratanto ci limiteremo ad osservare, come la presenza della Taeniopteris vittata Brong. e del Pterophyllum minus Brong. avvalorino questa classificazione, mentre $l^{p}$ importanza data alla Clathropteris meniscioides Brong., specie liassica rinvenuta pure ad Iloer, viene scemata dal fatto, chessa esiste isolata, e soltanto nella parte inferiore di quei depositi in una roccia diversa da quella in cui superiormente si trovano le altre piante.

\section{Calamites Reanii Bunbury.}

Tav. III. fig. 1.

G. caule decorticato, articulato, diam. circiter 16 centim., articulationibus valde tumidis, internodiis $8-9$ centim. Iongis, costis latis, tuberculis nullis.

Calamiles Beanii. Bunbury, Foss. plants f. the Jurass. strata of the Yorksh. Coast. Quart. Journ. Geol. Soc. of London. Vol. VII. 1851, pag. 189.

Calamites giganteus. BeAN in mss. Vid. Buns. loc. cit.

In arenaria carbonifera inferiori formationis oolithicae ad Scarborough Angliae.

Secondo il signor Bunbury, questa specie è particolarmente caratterizzata dalle larghe coste che la percorrono longitudinalmente, e dalla rilevante intumescenza delle sue arti- 
colazioni, che rende in quel punto il diametro del caule assai maggiore di quello sia negli spazj intermedj.

Dalla gentilezza di quel distinto Naturalista ebbi il disegno, ridotto alla metà della naturale grandezza di un esemplare posseduto dal sig. Bean. Però da questo disegno, che riportai alla fig. 1. della Tav. III., quale mi fu inviato, non si riscontra chiaramente espressa una parte dei caratteri che indussero il Bunbury a ritenerlo diverso dal Calamites arenaceus del Brongniart e da quello osservato in America negli strati carboniferi dell'pepoca oolitica nella Virginia orientale. Tuttavia vi si può riconoscere l'assenza dei tubercoli e della corteccia esterna, il diametro del caule che giunge ai sedici centimetri, e la lunghezza degl'internodj che non oltrepassa i nove centimetri.

Il sig. Bean, che nella sua numerosa raccolta di piante dell’ Oolite possede varj esemplari di questa specie, l'aveva distinta col nome di Calamites giganteus; ma il

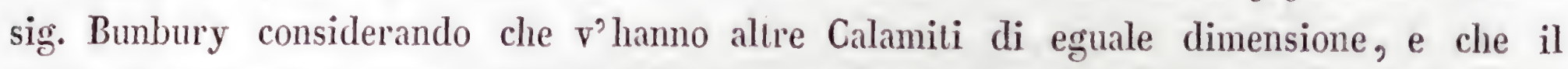
nome di Calamites gigas, dato dal Brongniart ad altra e ben diversa specie, potrebbe agevolmente confondersi con quello imposto dal sig. Bean alla presente, preferi apporvi il nome dello scopritore.

\section{Calamites lateralis Zign.}

Tav. III. fig. 3.

G. caule tereti semipollicem crasso striato, articulis 4-5 centim. circiter longis, discis inter articulos elevatis radiato-striatis, foliis verticillatis linearibus planis uninerviis basi contiguis.

Equisetum laterale. Pinluips, Geol. of Yorkshire, Pars I. pag. 125, tab. 10. fig. 13. - Lind. Hutr. Foss. Flor. of Gr. Brit. Vol. III. tab. 186.

Equisetites lateralis. Morris, Cat. Brit. Foss. pag. 8. - Göpr. in Brows, Gesch. d. Nat. pag. 15. - Uvg. Synops. pag. 28; Gen. et Spec. plant. foss. pag. 59. - Eтtixgsu. Monograph. Calam. in Beitr. z. Flor. d. Vorw. pag. 27.

Asterophyllites? lateralis. Buxbury, Foss. plant. from the Jur. strate of the Yorksh. Coast. in Quart. Journ. Geol. Soc. Lond. Vol. VII. pag. 189 (1851).

In arenaria carbonifera inferiori formationis oolithicae ad Haiburn Wyke, nec non ad White Nab prope Scarborough Angliae.

Il caule quasi cilindrico ha un diametro di undici millimetri circa, ed è percorso da strie longitudinali parallele, distanli fra loro poco più $d^{\text {s } u n ~ m i l l i m e t r o . ~ G l}$ internodi va- 


\section{$-47$}

riano nella lunghezza dai quattro ai cinque centimetri, e sono forniti, per lo più nel terzo inferiore, di un disco ombilicato nel centro, e segnato nei margini da strie disposte a raggio. Le articolazioni sono alcun poco rilevate, e lasciano vedere nel loro contorno una specie di brevissima guaina, che mi sembra prodotta dalla base delle foglie lineari piane uninervie che sono disposte a verticillo intorno all'articolazione. Queste ultime non oltrepassano in larghezza un millimetro, e giungono a due centimetri e mezzo di lunghezza.

Il Prof. Phillips, che diede pel primo la figura di questa pianticella, la riferisce al genere Equisetum. Gli Autori della Flora fossile dell'Inghilterra ne figurarono da poi un esemplare migliore, trovato dallo stesso Prof. Phillips a White Nab; e sebbene abbiano conservato il nome che le fu imposto da quest'ultimo, pure si mostrano poco persuasi che possa realmente appartenere agli Equiseti, e notano le analogie che mostra avere colle Calamiti.

Il sig. Bunbury nella sua dotta Memoria sopra le piante fossili degli strati giurassici della Contea di York prende ad analizzare i caratteri svelati da un esemplare assai più perfetto dei precedenti, esistente nella collezione del signor Bean a Scarborough; e dalle foglie lineari, piane, munite di una sola nervatura ch? escono a verticillo dalle articolazioni, viene indotto a collocare provisoriamente questa pianta fra le Asterofilliti. Non ommette però il Bunbury di ripetere egli pure l'osservazione dei signori Lindley e Hutton, che cioè essa si colleghi eziandio al genere Galamites mercè i dischi rilevati e radiati che si veggono sparsi quà e là sugli internodj.

Ora ritenendo che l'apparente r'udimento di guaina, visibile nella porzione ingrandita della nostra figura, sia dovuto alla persistenza della base connata delle foglie, la presenza di queste ultime esclude affatto l'idea che possa appartenere agli Equiseti; ed avendo ammesso coll' Ettingshausen la riunione delle Asterofilliti alle Calamiti, la pianta in discorso viene naturalmente a prendere il suo posto nel genere Calamites.

Questa specie sembra caratteristica dell’arenaria carbonifera inferiore dei depositi di Scarborough; arenaria che si ritiene interposta fra il terreno della grande Oolite ed il piano più basso dell' Oolite inferiore.

In quanto poi all' Equisetite trovato nell' arenaria liassica di Steierdorf nel Banato dall' Andrae, riferito alla specie presente, è da osservarsi che, meno i dischi radiati, in tutto il resto non offre caratteri sufficienti per identificarlo all' E. lateralis, almeno per quanto si può dedurre dai frammenti figurati nella Memoria dell ${ }^{p}$ Andrae. 


\section{Calamites Rogersii Bunbury.}

G. caule cylindraceo, cortice tenuissima, transverse plicato, diam. 12-24 centim., articulationibus distantibus, internodiis diametro caulis subaequalibus, costis creberrimis parallelis 1 millim. circiter latis, convexis, subcarinatis, tuberculis nullis.

Calamiles Rogersii. Buns. Foss. pl. from the Jur. str. of the Yorksh. coast. Quart. Journ. Geol. Soc. Lond. Vol. VII. pag. 190.

Calamites arenaceus. Rogens, Report. of Assoc. of American Geol. 1843, pag. 504. - Buns. Descript. foss, pl. from the Coal-field near Richmond, Virginia. Quent. Journ. Geol. Soc, Lond. Vol. III. pag. 284.

Calamiles Suckowii, var. $\delta$. Brosg. Itist. végét. foss. I. pag. 125, tab. 16. fig, 1.

In schistis et in arenaria cinerea inter strata carbonifera formationis oolithicae inferioris Americae ad Blackheath prope Richmond Virginiae.

Numerose porzioni del caule di questo Calamite si trovano di frequente negli schisti nerastri e nelle arenarie grigio-azzurrognole poco coerenti che accompagnano le roccie carbonifere della Virginia orientale, riferite alla parte più bassa dell’0olite inferiore. Gli esemplari che giaciono negli schisti sono coricati ed alquanto compressi; quelli che si osservano nelle arenarie sono in posizione verticale e cilindrici. Specialmente gli ultimi dánno a divedere numerose pieghe trasversali, probabilmente cagionate dalla pressione in senso verticale. Il caule giunge talvolta ad avere un diametro di ventiquattro centimetri, ed è ricoperto a tratti da sottile corteccia carboniosa assai fragile e liscia, $\mathrm{i}$ cui residui si scorgono riempiere $\mathrm{i}$ minuti solchi interposti alle numerose coste che longitudinalmente lo percorrono. Hanno queste nelle porzioni denudate un diametro di poco pii

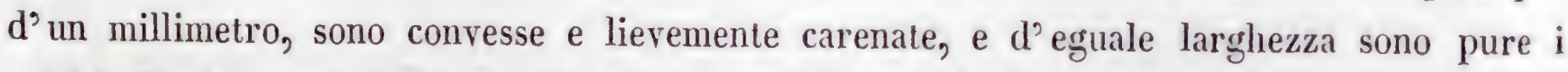
solchi che le separano. Le articolazioni sono distanti fra loro quanto è largo il caule, e non presentano traccia alcuna di tubercoli.

Il Prof. Rogers ed il sig. Bunbury riferirono con qualche dubio la presente specie al Calamites arenaceus del Brongniart, nello stesso tempo identificandolo alla varietà $\delta$ del Calamites Suckowii; varietà stabilita da quest ${ }^{2}$ ultimo illustre Naturalista sopra un esemplare proveniente dalle miniere di Richmond nella Virginia, inviatogli dal Prof. Silliman. Tuttavia tanto il Prof. Rogers quanto il signor Bunbury, notano alcuni caratteri che non permettono di adeguarla con piena sicurezza al Galamiles arenaceus; ed ultima- 
mente il signor Bunbury, accennando alle osservate differenze, propose per questa specie il nome di Calamites Rogersii. E, per vero, il Calamite in discorso, quantunque vario nelle dimensioni, raggiunge però sempre un diametro che supera $\mathrm{d}^{ }$assai il massimo finora conosciuto del Calamites arenaceus; nè si mostra giammai, come quest'ultimo, in alcuni punti cosi rigonfio da sembrare talvolta fusiforme. Le coste da cui è percorso non s'allargano alla base, ma corrono tutte eguali ed equidistanti fra loro in tutta la lunghezza degl'internodj, che per lo più sono lunghi quanto è largo il caule, mentre nel Calamites arenaceus havi ordinariamente fra le articolazioni una distanza assai maggiore del loro diametro. Aggiungeremo che le frequenti pieghe trasversali, da cui è segnato il Calamites Rogersii, ci palesano una consistenza assai più molle ed erbacea di quella del Calamites arenaceus. Per queste ragioni ho creduto di dover abbracciare $l^{9}$ opinione del Bunbury, separando la presente specie da quella a cui fu dapprima riferita, ed apponendovi il nome del Prof. Roger's.

\section{Calamites planicostatus Rogers.}

G. caule compresso articulato longitudinaliter sulcato, articulis cicatricibus circularibus instructis, costis planis basi convexiusculis, striis $1-2$ medio percursis, tuberculis nullis.

Calamites planicostatus. Rogers, On the age of the Coal Rocks of East. Virginia. Reports of the Assoc. Amer. Geol. 1843, pag. 305.

In argilla carbonifera formationis oolithicae inferioris Americae, frequenter occurrit prope James river in Virginia.

Un caule assai compresso, diviso in articolazioni prive di tubercoli, è l'apparenza che ci presenta questa specie negli esemplari meglio conservati. Esso è percorso in tutta la sua lunghezza da solchi poco profondi e paralleli, distanti l' uno dall'altro non più di due millimetri. Questi solchi sono continui, e si prolungano attraverso le articolazioni, solamente flettendosi alquanto o divergendo lateralmente in quel punto, e poscia riprendendo l'originario loro andamento. Le coste che stanno fra l'uno e l'altro solco sono liscie ed appianate, tranne presso le articolazioni, ove sono leggermente ed in modo irregolare convesse. Sulla parte loro mediana si scorge una o due finissime strie longitudinali. Le articolazioni sono spesso fornite di cicatrici circolari, che, secondo il Rogers, sarebbero le traccie del punto $d^{2}$ inserzione di appendici fogliacee. Queste cicalrici sono disposte ad intervalli di quindici millimetri all incirca.

Dalla generale compressione degli esemplari e dalla poca profondità dei solchi si prenderebbe quasi per una larga foglia striata; senonchè levando il sottile strato carbo- 
nizzato che cela le articolazioni, si scopre in modo non dubio la natura articolata propria di un caule.

Sembra che a questa specie abbia voluto alludere il sig. Bunbury nella sua Memoria sulle piante fossili della Virginia orientale, inserita nel Quarterly Journal of the Geological society of London (Vol. III. pag. $285, n^{\circ}{ }^{\circ} 9$ ), ove parla di alcuni fusti assai compressi, a coste striate, che riferisce semplicemente al genere Calamites, senz' apporvi alcun nome specifico. Egli propende a ritenerli spettanti al Calamites arenaceus del Brongniart, di cui sarebbero, secondo lui, giovani individui. Però nella presente specie i solchi continui che la segnano longitudinalmente, le strie che si osservano sopra il dorso delle coste appianate, e le circolari cicatrici, mi sembrano caratteri cle valgono a sufficienza per distinguerla dalla specie Brongniartiana.

\section{Gen. XVil. Spuevophymum Brong.}

Caules simplices vel ramosi articulati striati, foliis vestiti verticillatis senis, octonis, denis, duodenisve cuneiformibus; nunc integris apice truncatis, denticulatis, vel obovato rotundatis; nunc bilobis, lobis bipartitis vel laciniatis; nunc bifidis lobis linearibus angustis. Spicae cylindricae laterales vel terminales, squamis verticillatis.

Bnong. Classificat. tab. 2. fig. 8.; Prodr. pag. 68; Tabl. Gerb. végét. foss. pag. 52. - Enducu. Gen. plant. pag. 45. - Unc. Synops, pag. 115; Conspect. Flor. primord. in Chlor. Protog. pag. uir ; Gen. et Spec. plant. foss. pag. 69. - Etringsi. Monograph. Calam. pag. 20. - Geinitz, Verstein. der Steinkohlenform. in Sachsen, pag. 12. - Brows, Lethaca Geogn. Vol. I. pag. 105.

Rotularia. Stenns. Vers. Flor. Vorw. I. pag. xxxir.

Trizygia. Forbes Royle. Illustr. of the Bot. and Nat. Hist. of the Himalay. Mount. and Flor. of Cashmere. I. pag. xxix.*

Vertebraria. Forbes Royle in Op. cit. I. pag. xxix. ${ }^{*}$ - N' Coy, Foss. Bot. and Zoology of the Coal in Australia in Annals and Magaz. of Nat. Hist. Vol. XX. pag. 146.

\section{Sphenophyllum radiatum Ung.}

S. caule tereti flexuoso, verticillis foliorum contiguis senis vcl octonis, foliolis cuneiformibus apice truncatis integerrimis approximatis. 
Sphenophyllum radiatum. Ung. Gen. et Spec. plant. foss. pag. 71. - Ertingsh. Monograph. Calam. in Beitr. zur Flor, der Vorw. pag, 21,

Vertebraria radiata. Fonbes Royue. Illustr. of the Bot. of the Himalayan Mount. ete. 1. pag. xxix. II. tab. 2. fig. 5. 6. 7. - Uvg. Synops. pag. 114; Conspect. Flor. primord. in Chlor. Protog. pag, Lifr. - Brons. Ind. Palaeont. in Gesch. der Nat. 5. pag. 1264.

Sphenophyllum Indicum. UNG. Gen. et Spec. plant. foss. pag. 71.

Vertebraria Indica. Fonbes Royle in Op. cit. pag. xxix. II. tab. 2. fig. 1. 2. 5. - Uve. Synops. pag. 114; Conspect. Flor. primord. in Chlor. Protog. pag. LuI. - Brons. Ind. Palacont. in Gesch. der Nat. III. pag. 1564.

In schisto argillaceo et in arenaria formationis oolithicae inferioris ad Burdwan et Umret, nec non in collibus Mahádewa ad Nagpur Indiae.

Questa pianticella ci si presenta solto $\mathrm{l}^{\text {}}$ aspetto di un cilindro irregolare, avente un diametro non maggiore di due centimetri, ma cle talvolta è d'assai minore, ed in alcumi esemplari si scorge gradatamente diminuire, procedendo verso l'estremità superiore. Nel centro di questo cilindro scorre longitudinalmente il caule, rotondetto, flessuoso, dello spessore $d^{2}$ un millimetro o poco più, e da cima a fondo coperto tutto all'intorno da verticilli di foglie, sovraposti $1^{3}$ mo all'altro e contigui in guisa, che le pagine delle foglioline si toccano, e dánno alle piante, con questo combaciamento degli addensali verticilli, $l^{2}$ indicata apparenza di un cilindro. I verlicilli si compongono di sei ad olto foglioline cuneiformi, tronche all’ estremità, lunghe, secondo gli esemplari, dai quattro ai dieci millimetri, e larghe quasi altrettanto nella loro parte più ampia, ossia nel lato esterno. Queste foglioline lianno l'aspetto triangolare. Il lato che ne forma l'estremilà è un $\mathrm{po}^{9}$ curvo esternamente, e gli angoli ne sono smussati. All' incontro è acuto l'angolo interno, col quale $\mathrm{s}^{\mathrm{s}}$ inseriscono sul caule. Le foglioline, che, come dicemmo, sono contigue in senso verticale, lo sono pur anco nel senso orizzontale, e lasciano soltanto vedere le linee di separazione, che dánno un aspetto radiato all’ interna struttura, come si osserva considerandone il piano trasversale.

Io seguii l'Euingshausen, riunendo in una sola specie la Vertebraria radiata e la Vertebraria Indica di Forbes Royle, che l'Unger manteneva separate, ponendole però egli pure nel genere Sphenophyllum. Nè dalle figure del Forbes Royle, nè dalle frasi specifiche dell' Unger puossi dedurre alcun carattere che valga a distinguerle l'una dall'altra, eguali essendo in tutte due la forma e le dimensioni del caule, la distribuzione dei verticilli, la figura, il numero ed il modo d'inserzione delle foglioline. 


\section{Sphenophyllnm australe Ung.}

S. caule stricto, verticillis foliorum contiguis constanter octonis, foliis cuneiformibus apice truncatis integerrimis obsolete concentrice plicatis, nervis furcatis laeviter impressis.

Sphenophyllum australe. Usg. Gen. et Spec. plant. foss. pag. 72. - Etrivgsir. Monograph. Calam. in Beitr, zur Flor, der Vorw. pag. 23.

Vertebraria australis. M' Cor. On the foss, Bot. and Zool. of the Roclis associated with the Coal of Australia, in Annals and Magaz. of Nat. Hist. 1847. Vol. XX. pag. 152, tab. 9. fig. 1.

In schisto argilloso albescente ad Malubimba Novae Hollandiae australis.

In questa specie il caule è assai più soltile che nella precedente, e nell'esemplare illustrato dal $\mathbf{I}^{2} \mathrm{Coy}$ non mostra alcuna flessuosità od ineguaglianza, ma corre diritto nel centro del cilindro prodotto dagli appressati verticilli delle foglie. I diversi frammenti di questa pianta raccolti finora hanno varia lunghezza, ma un diametro quasi costante di quattordici millimetri. Le foglioline, pur cuneiformi e tronche all' estremità, sono alquanto più ristrette verso la base di quelle della Vertebraria radiata, e si osservano sempre in numero di otto per ciascun verticillo. Esse sono percorse da vene esilissime, dicotome, e raggianti dal punto d'inserzione delle foglie, e si mostrano segnate da alcune pieghe concentriche appena discernibili, alle quali corrispondono le strie longitudinali che si scorgono perpendicolari al piano dei verticilli nella sezione verticale degli esemplari.

Bene considerando le due specie testè descritte, non trovo motivi sufficienti per ritenerle separate dagli Sfenofilli, e mantenere il genere Vertebraria, fondato da Forbes Royle, che si limitò a dare le figure delle sue due specie indiane senz' apporvi alcuna descrizione, e senza indicare i caratteri del nuovo genere da lui proposto.

$\mathrm{M}^{2} \mathrm{Coy}$, che si occupò della specie trovata in Australia, caratterizza il genere Vertebraria nel modo seguente. "Caule sottile, circondato da verticilli addensati; foglie " cuneiformi, munite di nervature dicotome." Premette però, come un accurato studio instituito sovra parecchi esemplari lo induca a ritenerlo molto affine agli Sfenofilli; colla sola differenza, che nelle Vertebrarie i verticilli composti dalle foglioline cuneiformi sono sovraposti l' uno sull'altro, e talmente vicini da procurare alla pianta l'aspelto di un cilindro continuo. Unger ed Ettingshausen collocano a dirittura le Vertebrarie nel genere Sphenophyllum; ed io non esitai punto di uniformarmi al loro parere anche per non moltiplicare i nomi sopra dati così incerti. 


\section{- ร3 -}

Queste forme sono interessanti, perchè proprie finora soltanto delle roccie che accompagnano i carboni fossili delle Indie e dell'Australia; depositi che havi ogni ragione di credere sieno collocati nella parte inferiore del terreno oolitico; ed ove l'appartenenza di queste specie agli Sfenofilli venisse da future osservazioni convalidata, ci porgerebbero, con quella che segue, il primo indizio della esistenza di questo genere nell’0olite.

\section{Sphenophyllum Trizygia Ung.}

S. caule articulato, verticillis foliorum senis per paria approximatis, paribus oppositis aequalibus internodio longioribus, tertio minore, foliis obovalo-oblongis intcgerrimis nervosis, nervis furcatis subparallelis.

Sphenophyllum Trizygia. Ung. Gen. et Spec. plant. foss, pag. 74. - Etringsu. Monogr. Calam. in Bcitr. zur Flor. der Vorw. pag. 25.

Trizygia speciosa. Forbes Royle. Ill. of the Bot. and Zool. of the Himalayan Mornt. pag. xxrx." tab. 2. fig. 8. - UxG. Synops. pag. 114.; Conspect. flor. primord. in Chlor. Protog. pag. LIIr. Bronv. Ind. Palacont. in Gesch. der Nat. III. pag. 1295.

In schistis argillosis ad Burdwan et Umret, ac in arenaria collium Mahádewa ad Nagpur Indiae.

L'esemplare figurato dal Professore Forbes Royle ci presenta una porzione di questa pianta della lunghezza di quindici centinetri e mezzo, alla quale manca l'estremità, ma ch'è sufficiente a farci conoscere i caratteri principali che servono a distinguerla.

Il caule apparisce articolato, ed ha un diametro di poco più d'un millimetro, clie verso la parte superiore insensibilmente decresce. Le articolazioni in cui è pârtito sono più distanti fra di loro nella parte inferiore; perciò gl' internodj hanno ivi una lunghezza di oltre due centimetri, mentre procedendo verso l'estremità gradatamente si accorciano, e giungono appena ad un centimetro e mezzo; ed un'eguale proporzionata decrescenza si osserva anche nei verticilli e nelle foglioline di cui sono composti. Ogni verticillo consta di sei foglioline, disposte due a due in tre coppie, due laterali opposte e più lunghe, e la terza minore. Le foglie maggiori sono obovato-oblunghe, e giungono nei verticilli inferiori a tre centimetri di lunghezza e ad uno di larghezza nella parte loro piu ampia; quelle del pajo minore sono obovate soltanto, larghe pure un centimetro, e lunghe circa un centimetro e mezzo; ed ancor esse diminuiscono in proporzione progredendo verso l'estremità, ove le più grandi non oltrepassano i due centimetri in lunghezza, e le minori si limitano ad uno: cosicchè si può ritenere costante il falto, che in ciascun verticillo le foglioline delle coppie maggiori sieno lunghe precisamente il doppio di quelle che compongono la coppia minore. 
Le nervature di ciascuna fogliolina si dipartono in modo flabellato dal punto d'inserzione della stessa sul caule, indi si biforcano due o tre volte con lieve divergenza, e scorrono quasi parallele verso l'estremità rotondata delle foglie.

L'illustre Brongniart, parlando di questa pianta, così s'esprime all'Articolo Sphenophyllum del suo Tableau des Genres des végétaux fossiles, pag. 53: "Le genre "Trizygia de Royle, fondé sur une seule espèce (Trizygia speciosa), observée par ce "savant dans les mines de houille de l'Inde, me parait seulement une espèce remar"quable du genre Sphenophyllum." Il chiarissimo Prof. Unger, che nelle sue publicazioni anteriori aveva conservato il genere Trizygia di Forbes Royle, senza però indicarne i caratteri, nell'Opera intitolata Genera et Species plantarum fossilium, publicata l'anno 1850 , collocò la Trizygia speciosa tra gli Sfenofilli; e l'Ettingshausen nel suo recente dotto lavoro monografico sulla Classe delle Calamarie segue il parere dell' ${ }^{\text {Unger. }}$

All' incontro i signori Hislop ed Hunter nella loro importante Memoria sulla Geología delle vicinanze di Nagpur nell' India centrale non sembrano favorire questa riunione, forse perchè, ritenendo il genere Sphenophyllum esclusivo del vero terreno carbonifero, la pre-

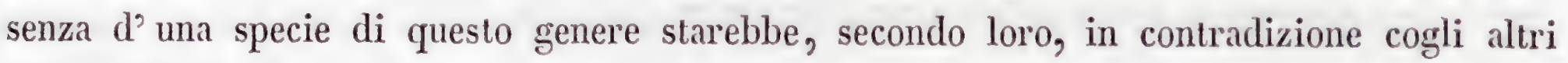
caratteri paleontologici che indussero que due valenti Naturalisti a classificare con tutta ragione i carboni fossili di Burdwan, gli schisti bituminosi di Umret, e le arenarie fitolitifere dei contorni di Nagpur, come spettanti alla formazione oolitica. Ma altri generi dell'epoca carbonifera sono pure comuni a questa formazione, e la pianticella che descrivemmo ha troppo strette analogie cogli Sfenofilli per polernela ragionevolmente separare.

Questa forma, propria delle Indie, non si è ancora veduta ripetersi nei terreni oolitici d'altre parti del globo.

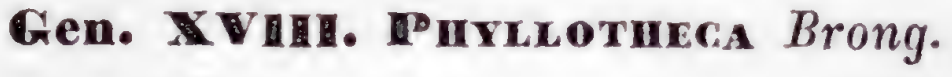

Gaulis rectus articulatus, simplex vel ramosus. Rami supra nodos orientes in eodem plano haud dispositi, superficie laevi vel longitudinaliter sulcata. Articuli vaginati, vaginis in laminas lineares enervias vel nervo medio plus minusve distincto percursas, Ionge productis. Inflorescentia extremitate ramulorum quorumdam verticillata.

Brong. Prodr. pag. 151.; Tabl. des Gen. péyét. foss. pag. 54. - N' Cox. Foss. Bot. and Zool. of the Rocks assoc. with the Coal of Australia in Annals and Magaz. of Nat. Iist. Vol. XX. pag. 152. - Uvg. Synops. pag. 35. ; Conspect. Flor. primord. in Chlor. Protog. pag. xxxir.; Gen. et Spec. plant. fass. pag. 72. - Erringsu. Monograph. Calam. in Beilr. zur Flor. der Vorw. pag. 24. 


\section{Phyllotheca australis Brong.}

Ph. caule erecto simplici articulato laevi vel substriato, vaginis angustis striatis, internodiis brevioribus, laminis angustis bis duplo longioribus acutiusculis enerviis.

Phyllotheca australis. Bnong. Prodr. pag. 152. - Monns in Strzelecki, Physic. descrip. of New South Wales and Van Diemen's Land. 1845, pag. 250. - M'Cox. On the Foss. Bot. und Zool. of the Rocks assoc. with the Coal of Australia in Annals and Magaz. of. Nat. Hist. 1847, Vol. XX. pag. 156. - Ung. Synops. pag. 55 ; Conspect. Flor. primord. in Chlor. Prolog. pag. xxxiI.; Gen. et Spec. plant. foss. pag. 75. - Brons. Ind. Palaeont. in Gesch. der Nat. III. pag. 971. - Etringsu. Monogr. Calam. in Beitr. zur Flora der Vorw. pag. 24.

In argilla carbonifera formationis oolithicae inferioris ad Newcastle et ad Hawkesbury river Novae Hollandiae orientalis.

Questa specie ha il caule semplice, eretto, articolato, munito alle articolazioni di guaine anguste ed approssimate al caule, il lembo delle quali si risolve in foglie lineari alquanto allungate. Queste espansioni fogliacee delle guaine sono piane, senz'alcun indizio di nervatura, e nell' estremità leggermente acuminate. La loro lunghezza raggiunge il doppio di quella delle guaine, e variano nella direzione, talvolta essendo erette, talvolta patenti, e spesso rivolte all'ingiù. Le guaine sono segnate da lievi solcature longitudinali, che svaniscono verso la base, e corrispondono agl' intervalli fraposti alle foglie. Il caule, secondo Brongniart, sarebbe liscio soltanto nello stato giovane, giacchè nei frammenti che hanno maggior diametro, e che, a parer suo, apparterebbero ad individui più adulti, si osservano delle strie regolari che rammentano quelle delle Calamiti. Il M' Coy riferisce invece questi frammenti alla specie che sussegue.

Fino dal 1828 il Brongniart fondava il genere Phyllotheca sopra alcuni esemplari comunicatigli dal Prof. Buckland, e provenienti dalle miniere di carbon fossile di Hawkesbury river presso Port Jackson nella Nuova Olanda.

Il carattere che vale a ricisamente distinguere questo genere da tulti gli altri compresi nella Classe delle Calamarie consiste nelle foglie lineari allungate ora diritte, ora patenti, e spesso assai riflesse, in cui si divide il margine superiore della guaina. Però i signori Lindley e Hutton nella Fossil Flora of Great Britain, pag. 89 del Vol. II., ove parlano della pianta da essi descritta sotto il nome di Hippurites gigantea, agginngono alcune osservazioni sulla Phyllotheca australis del Brongniart, dalle quali si de- 


\section{$-56-$}

sume ch'essi calcolano erroneo il carattere da quest'ultimo assegnato alle Filloteche, ed opinano invece che le foglioline sieno inserite sotto la base della guaina, la quale sarebbe cosi esternamente circondata dal verticillo delle foglie stesse.

Il Prof. Unger nella Synopsis plantarum fossilium, stampata nel 1845, e nel Conspectus Florae primordialis, che fa parte della sua Chloris Protogaea, piegandosi al parere degli autori della Flora fossile della Gran-Bretlagna, definisce il genere Phyllotheca nel modo seguente. "Caulis simplex, reclus, articulatus, vaginatusque. Folia ver" ticillata linearia enervia contracta vel expansa, vaginas articulorum strictas circumdan"tia." All' incontro il sig. Morris, che illustrò le piante fossili della Nuova Olanda, annoverate nell' Opera dello Strzelecki, che ha per titolo Physical Description of New South Wales and Van Diemaa's Land, publicata nel 1845, descrive la Phyllotheca australis assegnandole i caratteri già indicati dal Brongniart; ed il M' Coy nel suo bel lavoro sui fossili animali e vegetali delle roccie associate ai carboni fossili dell'Australia prova chiaramente l'aggiustatezza delle vedute del Naturalista francese, e svela come i signori Lindley e Hutton sieno stati indotti in errore da qualche frammento capovolto di quegli esemplari a foglioline molto riflesse, $\mathrm{i}$ quali, osservati in posizione inversa, possono appunto destare l'idea che queste ultime sorgano dalla base, piuttostochè dalla estremità delle guaine.

Nelle altre specie descritte e figurate dal $M^{\prime}$ Coy si riconosce sempre la costanza del carattere generico desunto dalla guaina, quale fu indicato dal Brongniart fino dal $\$ 828$ nel Prodrome d'une Histoire des végétaux fossiles, e recentemente nel suo Tableau des Genres des végétaux fossiles, pag. 54. Perciò 1’Unger nell’opera intitolata Genera et Species plantarum fossilium riformò in questo senso la frase caratteristica del genere, modellandola su quella proposta dal $M^{\circ}$ Coy.

Il $M^{5}$ Coy nell' Opera che cilammo aggiunge altre osservazioni, per le quali troverebbe nelle Filloteche molta affinità colle Casuarine dell'epoca attuale. Accenna in primo luogo ai rami inseriti al di sopra delle arlicolazioni, ed uscenti dalla parte del caule ch’̀̀ investita dalla guaina; indi all' infiorescenza, che riscontrò composta di articolazioni poste a brevissimi intervalli, e ricoperte da guaine lunghe quanto gl' internodj, le quali negli orli sono guarnite, a $\mathrm{mo}^{\circ}$ di frangia, da verticilli di corpi bicellulari, in cui egli crede ravvisare organi analoghi alle antere delle Casuarine.

A questo proposito troverei di osservare che il modo di ramificarsi delle Filloteche, se è diverso da quello delle Equisetacee, non lo è gran fatto da quello delle altre Calamitee; e clie all'incontro la infiorescenza, quale ci viene rappresentata dalla figura del II' Coy, ricorda molto più l'amento degli Equiseli, che il fore delle Casuarine. Bastera prendere la fruttificazione di un Equiseto giunta a malurità, quando i riceltacoli ne sono sollevati, e lievemente comprimerla, per ottenere un profilo eguale a quello che ci pre- 


\section{$-57$}

senta la figura di $M^{2}$ Coy (vedi Memoria inserita negli Annals and Magaz. of Nat. Hist. Vol. XX. tav. 11. fig. 1.); ed è molto più verisimile che i corpi bicellulari veduti da quel Naturalista rappresentino i resti degli sporangi, anzichè organi analoghi alle antere, la cui conservazione allo stato fossile è per lo meno assai rara.

Anche la forma molto prolungata dalle guaine e la consistenza erbacea delle Filloteche concorrono ad allontanarle ancor più dalle Casuarine, mentre gli altri caratteri paleserebbero in esse un genere che anderebbe a formare quasi $1^{2}$ anello di congiunzione fra le Calamilee e le Equisetacee.

Il genere Phyllotheca, trovato da prima soltanto nella formazione in cui giaciono i carboni fossili della Nuova Olanda, sembrava esclusivo di quella regione; ma di poi si rinvennero dei resti spettanti a questo genere anche nei terreni analoghi delle Indie, ove il sigg. Ilunter trovò con essi l'Equisetum laterale di Phillips, che io collocai provisoriamente nel genere Calamites, ma che ove si riscontrasse decisamente fornito di una guaina congiunta alla base delle foglie (come arrei motivo di sospettare), potrebbe anche esso prender posto tra le Filloteche.

A questo genere io riferisco pure alcune forme che si osservano nei terreni oolitici delle Alpi Venele, e che descriverò più imnanzi.

\section{Phyllotheca ramosa $M \prime$ Goy.}

Ph. caule cylindrico, erecto, articulato, ramoso, laevi vel substriato, diam. 5-10 millim.; ramis alternis supra nodos orientibus, cernuis; vaginis internodio dimidio brevioribus; laminis circiter 24, tenuibus, linearibus, angustissimis, planis, inaequalibus, vaginas duplo vel triplo superantibus, saepe reflexis; nervo medio tenuissimo vix distincto.

Phyllotheca ramosa. M' Cox. Foss. Bot. and Zool. of the Rocks assoc. with the Coal of Australia, in Amnals and Magaz. of Nat. Hist. Vol. XX. pag. 156, tab. 11. fig. 2-5. in Supplem. - Uva. Gen. et Spec. plent. foss. pag. 75. - Etringsir. Monogr. Calam, in Beitr. zur Flor. der Vorw. pag. 24.

In argilla scissili albescente seriei oolithicae inferioris ad Mulubimba Novae Hollandiae australis.

Questa specie, descritta e figurata da $\mathrm{NI}^{9} \mathrm{Coy}$, ha un caule ramoso, cilindrico, articolato, che varia nel diametro all’ incirca dai cinque ai dieci millimetri, ed è segnato da lievi strie longitudinali molto avvicinate e parallele, che nelle porzioni decorticate si osservano soltanto in prossimità delle articolazioni. Superiormente a queste ultime sono 
alternamente inseriti i rami pure cilindrici ed articolati, i quali curvandosi all'ingiu, si veggono pendere da ambe le parti del caule, ed hamno un dianetro che non oltrepassa la metà dello spessore di quest’ultimo. La lunghezza degl'internodj equivale all’ incirca al doppio del diametro, e ciò tanto sul caule che nei rami. Le guaine, che giungono quasi alla metà değl'internodj, sono striate, e si dividono superiormente in foglioline piane, lineari, sottilissime, $d^{\prime}$ ineguale lunghezza, percorse da un esilissimo nervo mediano appena discernibile colla lente. Queste espansioni fogliace delle guaine, che in qualche verticillo osservai giungere al numero di ventiqualtro, sono ritte sul caule, patenti o riflesse sui rami, a seconda della loro posizione, scorgendosi nella porzione pendente di questi ripiegate verso la base della guaina, e quindi rivolte in senso inverso alla direzione dei rami. I verticilli sono ellitlici, come nelle Annularie, le foglie di due opposti punti della circonferenza essendo molto più lunghe delle altre.

A proposito della Phyllotheca australis abbiamo notato come i frammenti di caule segnati da strie fossero dal Brongniart riferiti ad individui piu adulti. II' Coy ritiene al contrario che i cauli si mostrino privi di strie non già per effelto di giovane età, ma bensi quando si osservino sopra esemplari privi della corteccia esterna. Anche da ciò egli trae argomento per far rilevare l'analogia che gli sembra esistere tra le Filloteche e le Casuarine, rimarcando come in queste $\mathrm{i}$ solchi non sieno cosi profondi come negli Equiseti, e non lascino alcun segno sul caule, levata che ne sia la corteccia. Tultavia se è vero che nelle Casuarine il caule denudato dalla corteccia non palesi traccia dei solchi interposti alle coste prominenti, ond'è segnata quest'ultima; però vi si distingunono delle minutissime strie longitudinali, simili a quelle che percorrono nello stesso senso tutta la corteccia. Di più, nelle Casuarine le coste di questa alternano al punto di congiunzione delle articolazioni in guisa che $\mathrm{i}$ solchi dell'internodio inferiore corrispondono perfettamente alle coste dell' internodio superiore; il che non si scorge nelle Filloteche.

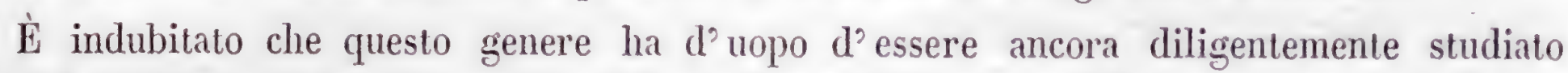
sopra buon numero di esemplari, onde meglio chiarirne le affinità. Fratanto per le cose esposte non mi sembra che i caratteri fin qui riscontrati nelle Filloteche sieno sufficienti per dimostrarle analoghe alle Casuarine.

Questa specie è comune nell'argilla scissile biancastra e poco coerente presso Mulubimba, in quella parte della Nuova Olanda, cui gl'Inglesi diedero il nome di Nuova Galles meridionale. 


\section{Phyllotheea Hookeri $M^{\prime}$ Goy.}

Ph. caule simplici, longitudinaliter grosse sulcato et striato; vaginis amplis, laxis, subinfundibuliformibus, striatis, internodiorum longitudine caulem omnino obtegentibus; laminis plurimis elongalis, vagina duplo longioribus, crassis, angustis; nervo prominulo valido medio percursis.

Phyllotheca IIookeri. M' Coy, Foss. Bot. and Zool. of the Rocks assoc. with the Coal of Australia, in Annals and Magaz. of Nat. Hist. Vol. XX. pag. 157, tab. 11. fig. 4. 5. 6. 7. in Supplem. Uxg. Gen. et Spec, plant. foss. pag. 75. - Ertixgsu. Monogr. Calam. in Beitr. zur Flora der Vorw. pag. 24.

In arenaria ad Clark's Ilill, in schistis siliceis ad Arowa, nec non in argillosis ad Hulubimba Novae Hollandiae australis.

Questa specie si distingue agevolmente da tutte le altre a cagione delle grandi guaine infundibuliformi, che libere e sciolte nella parte superiore ricoprono il caule per tutta la lunghezza degl'internodj. Fra i molti esemplari esaminati da $M^{2}$ Goy non ve $n^{2}$ ha alcuno che mostri indizio di ramificazione. Il caule, che in qualche saggio assai compresso raggiunge un diametro di cinque centimetri, si palesa solto le guaine segnato da coste ottuse e da solchi longitudinali e paralleli, che s'interrompono ove finisce l'internodio ́vedi $\mathrm{M}^{2} \mathrm{Coy}$, loc. cit., Suppl. tab. 11. fig. 7). Le guaine, che rapidamente s'allargano verso la loro estremità, sono marcate da minute strie, distanti l’una dall’altra appena un millimetro; e le foglioline, che si espandono numerose dal loro lembo superiore, sono lineari, di consistenza crassa, ritle ed avvicinate fra loro presso alla base, e superiormente alquanto divaricate e patenti, lunghe il doppio della guaina, e munitc di un nervo sporgente ben distinto, che ne percorre la parte mediana (vedi $\mathrm{M}^{2} \mathrm{Coy}$, loc. cit., Suppl. tab. 11. fig. 6).

Anche i saggi di questa specie abbondano nell’ argilla di Mulubimba, come pure negli schisti silicei di Arowa e nell' arenaria di Clark's IIill, roccie tutte che spettano al gruppo dell'polite inferiore.

\section{Ehyllotheea Erongniartiana Zign.}

Tay. 7 .

Ph. caule stricto, articulato, ramoso striato, diam. 2-millim.; internodiis 10millim. longis, superioribus brevioribus; ramis erectis, supra nodos et intra vaginas orientibus; vaginis laxis subcampanulatis, margine expansis, 2-millim. lon- 
gis; laminis 6-8 internodiorum longitudine vel paullo brevioribus, enerviis, inaequalibus, divaricatis, saepissime leviter incurvis.

In saxo calcareo griseo ex stratis superioribus seriei oolithicae inferioris (Étage Bathonien clarissimi A. d'Orbigny) ad Vallem Zuliani prope Roverè di Velo in Provincia Veronensi.

Il caule di questa specie, $\operatorname{cl}^{\prime}$ è segnato per lo lungo irregolarmente da minutissime strie molto avvicinate, ci si mostra eretto, articolato e sottile, non oltrepassando, sugli esemplari da me finora osservati, il diametro di due millimetri. Esso è fornito di ramificazioni semplici, distanti l'una dall'altra dai due ai tre centimetri. Gl' internodj sono lunghi all'incirca dieci millimetri; ma gradatamente s'abbreviano, procedendo verso le estremità della pianta, ove le articolazioni sono disgiunte fra loro dai due ai tre millimetri soltanto. I rami sorgono al di sopra delle articolazioni, e $\mathrm{s}^{9}$ inseriscono sul caule in quella parte ch`è rivestita dalla guaina, sicchè reggonsi uscire dall’ interno di questa (redi Tav. VII. fig. 3). Essi pure corrono ritti all' insù, scostandosi pochissimo dal caule, e si assottigliano verso $\mathrm{l}^{2}$ apice. Le guaine, di forma campanulata, sono brevissime, misurando appena due millimetri in lunghezza. Verso il margine si espandono, e dámno origine a sei od otto sottili lacinie d'ineguale lunghezza, a base allargata, discoste fra loro, che lievemente si curvano verso l'apice (vedi Tav. VII. fig. 7). Fra queste le più allungate raggiungono la lunghezza dell' internodio.

Questa pianticella, della quale non di rado si trovano i resti nel Veronese in Valle Zuliani, presso Roverè di Velo, nelle stratificazioni dell' 0 olite, ch' io ritengo rappresentare appo noi il piano Batoniano dell'Orbigny, si distingue agevolmente dalle altre specie per la sottigliezza del caule, per le ramificazioni erette e non ricurve che lo adornano, per la brevità delle guaine, e per lo scarso numero di foglioline in cui si risolve il margine. Io la intitolai all'illustre fondatore del genere. Tenue tributo d'omaggio e di riconoscenza verso un tanto uomo, clie con rara cortesía e liberalità mi fu largo di consigli e di preziose osservazioni intorno alle piante da me scoperte nell' Oolite delle Alpi Venete.

\section{Phyllotheca equisetiformis Zign.}

Tav, VIII.

Ph. caule erecto, articulato, simplici, substriato, diam. 4-8 millim., apice attenuato; internodiis inferioribus longitud. 20-millim., superioribus 10-millim. longis, versus apicem caulis sensim brevioribus; vaginis 3-millim. circiter lon- 
gis cauli arcte adpressis, superioribus approximatis, terminalibns confertis; lacinis $12-24$ aequalibus, rigidiusculis, crassis, enerviis, striatis, incurvis, 5-8-millim. longis, obtusis, deciduis.

Cum praecedente ad Vallem Zuliani prope Roverè di Velo, nec non in loco dicto i Pernigotti ad Vallem Tanara Provinciae Veronensis in eadem formatione.

Il caule articolato della presente specie si eleva ritto e senza ramificazioni, mantenendosi del diametro di otto millimetri per un tratto di venti centimetri all' incirca. Indi gradatamente diminuisce di spessore fino ai quattro millimetri, e poscia procede, assottigliandosi sempre più fino all'apice. Gl' internodj nelle porzioni di uniforme larghezza sono eguali fra loro, e segnati da minutissime strie appena visibili coll’ajuto della lente. Le articolazioni nella parte inferiore del caule sono discoste $l^{1}$ una dall'altra venti millimetri; superiormente questa distanza si riduce a dieci, e poscia va sempre diminuendo fino all' estremità della pianta. Le brevi guaine, di cui sono fornite, misurano tre millimetri in lunghezza, e sono affatto addossate al caule. Nella porzione terminale di questo per la brevità degl' internodj le vagine sono così approssimate in senso verticale da nascondere il caule stesso. Le lacinie cl' escono dal lembo superiore della guaina in numero di ventiquattro all'incirca nei frammenti che hanno maggior diametro, e di dodici nei più angusti, sono avvicinate alla base, eguali in ciascun verticillo, e lunghe all’ incirca dai cinque agli otto millimetri. Hanno un diametro di poco più $d^{3}$ un mezzo millimetro, ed una consistenza crassa ed alquanto rigida. Non mostrano alcun indizio di nervatura, ma sono invece segnate da lievi strie longitudinali. S' incurvano tutte più o meno verso il caule, e terminano ottusamente. Nei saggi spettanti alle parti inferiori di questa specie, e generalmente sulle porzioni più grosse del caule, mancano quasi sempre le lacinie, della cui anteriore presenza rimangono soltanto le traccie sull' orlo della guaina. All’ incontro gli esemplari che ci porgono i frammenti piu sottili delle parti terminali, o di quelle poste vicino alle estremità, si palesano sempre forniti dei verticilli formati dalle lacinie delle guaine, $\mathrm{i}$ quali per $\mathrm{l}^{\text {}}$ avvicinamento già accennato di queste ultime compariscono addossati l'ano sull'altro. (Vedi Tav. VIII. fig. 6.)

Sono frequenti i resti di questa specie nella zona calcarea fitolilifera dell’ Oolite veneta, ch’ io adeguai negli anteriori miei scritti al piano Batoniano dell’ Orbigny. I luoghi ove maggiormente abbondano trovansi nel Veronese, e precisamente nella Valle Zuliani presso Roverè di Velo, e nella Valle Tanara nel monte appellato Pernigotti.

Più comuni sono i frammenti del caule a diametro maggiore, colle guaine prive deile foglioline ormai cadute, ed è agevole confonderli col caule degli Equiseti, di cui allora 
presentano l'aspetto. Per questa simiglianza fui tratto ad applicare il nome di Phyllothect equisetiformis a questa specie, onde con ciò porre in avvertenza coloro che s'imbattessero in questi frammenti. Basterà esaminare con buona lente il margine della guaina per ravvisarvi i segni lasciati dalle lacinie.

Per le cose fin quì dette io credo che le due forme vegetali testè descritte non possano ad altro genere essere riferite. I caratteri desunti dalla guaina e dal modo di ramificazione mi sembrano chiaramente indicare la loro spettanza al genere Phyllotheca, che proprio dell' 0 olite, e circoscritlo finora ai depositi dell'Australia, fu recentemente osservato in quelli delle Indie dai signori IIislop e IIunter, però senza indicare la specie, e che si mostrerebbe per la prima volta in Europa nell' Oolite del Veneto con queste due specie certamente distinte dalle conosciute.

\section{Ordo VII. EquisetaceaE. \\ Gen. XX. EQuisetites Sternb.}

Spica terminalis, globosa, vaginae supremae semi-immersa, demum libera, squamosa, squamis valde approximatis peltatis pentagonis, concaviusculis subverticillatis. Caulis fistulosus cylindricus laevis vel Iongitudinaliter sulcatus striatusve, articulatus, articulis saepius introrsum concentrice striatis, simplex rel sub articulis ramosus, vaginatus; raginis sub articulationibus insertis erectis, multidentatis vel multifidis.

Stersb. Vers. Flor. Vorw. II. pag. 45. - Uxg. Synops. pag. 26; Gen. et Spec. plant. foss. pag. 55. - Etrngsu. Monogr. Calam. in Beitr. zu Flor. der Vorw. pag. 25.

Oncylogonatum. Koevig. Transact. Soc. Geol. Lond. Series II. Tom. II. pag. 500.

Equisetum. Broxg. Hist. vég. foss. I. p. 114 (pro parte). (Vid. Tabl. des Gen. vég. foss. p. 47.)

\section{Equisetites Bunburyanus Zign.}

Tav. III. fig. 2. 4. b̆. 6. Tav. IV. e V.

E. caule erecto ramoso, levi, diam. circ. 4-16 millim., sulcis 8-10 longitudinaliter instructo; internodiis 2-6 centim. Iongis, aequalibus, inferioribus brevioribus; vaginis subcampanulatis cauli adulto adpressis, 4-6 millim. circiter longis, striatis; dentibus minutis plurimis (20-30?) lanceolatis brevibus, marginatis, obtuse mucronatis. 


\section{$-63-$}

Equisetites elongatus Zign. (non Sternb.). Mém. terr. jur. Alp. Ven. 1852, pag. 10; Bull. Soc. Geol. Franç. Series II. Vol. XI. pag. 291; Leonh. Bronn. Neu. Jakh. für Min. Geogn. Geol. 1854, pag. 34.; Quurt. Journ. Geol. Soc. Lond. Vol.. X. n. 59.

In calcarea scissili grisea vel lutescente ex stratis Bathonianis in monte dicto Pernigotti ad Vallem Tanara, nec non ad Vallem Zuliani prope Roverè di Velo, et ad montem Raut in Provincia Veronensi.

Questa specie si distingue principalmente per avere un caule liscio, eguale, talvolta ramificato, e percorso in tutta la lunghezza degli esemplari più adulti da alcune pieghe o finissime solcature parallele. Sulla parte che non è nascosta dalla roccia ne contai da quattro a cinque, e suppongo che altreltante debbansi trovare sul lato opposto. Questi solchi sono discosti $l^{\text {' }}$ uno dall'altro all' incirca dai due ai tre millimetri. Secondo l'età varia il diametro del caule da' sei $a^{5}$ sedici millimetri; e cosi pure la lunghezza degl' internodj, che a seconda di quella misurano due, quattro o sei centimetri, ma sempre in guisa che il diametro corrisponda all’incirca al quarto della distanza che separa le articolazioni, le quali però presso alla base della pianta sono molto più avvicinate, come si scorge negli Equiseti virenti. Qualche variazione cagionata dall'età si osserva pure nelle guaine, essendo alcun poco campanulate, e non più lunghe di quattro millimetri negli esemplari minori; affatto appressate al caule, e lunghe dai quattro a' sei millimetri in quelli di un diametro maggiore. Sono esse regolarmente segnate da strie ben marcate, fra loro discoste poco più di mezzo millimetro, e contornate da minutissimi denti lanceolati brevissimi, a base allargata, che esaminati colla lente si mostrano lievemente marginati e brevemente mucronati. Dal novero di quelli che si possono vedere sulla superficie scoperta degli esemplari calcolo per approssimazione, che, secondo l’età di quesli, possano essere da renti a trenta tutto all' intorno. (Tedi $1^{2}$ ingrandimento alla fig. 4. della Tav. III.).

Negli elenchi delle piante oolitiche del Veneto, che inserii nelle publicazioni citate piu sopra, io aveva indicalo questa specie col nome di Equisetites elongatus, non avvertendo come lo Sternbergy aresse dato un'eguale denominazione ad altra e ben diversa specie. Per questo motivo doveui cangiare un nome che avrebbe recato confusione, e colsi questa opportunita per dedicarla al chiarissimo sig. C. J. F. Bunbury, dalla cui gentilezza mebbi parecchie importanti comunicazioni riguardo alla Flora dell’0olite.

Questa specie, la quale trovasi abbondare nei deposili spettanti al piano Batoniano del monte Pernigotti e della Valle Zuliani nel Veronese, fu recentemente scoperta eziandio nel monte Raut della stessa Provincia. Gli esemplari più grandi, figurati nella Tav. V.g provengono da quest'ultimo luogo. 


\section{Equisetites Veronensis Zign.}

Tav. VI.

E. caule erecto, cylindrico, ramoso?, striato, diam. 2 centim., internodiis aequalibus 4-5 centim. circiter longis; vaginis cauli adpressis longitud. 8-10 millim. profunde striatis, costatis; costis $1 .{ }^{1 / 2}$ millim. fere latis apice conicis; dentibus 26? remotiusculis, sinubus rotundatis divisis, subulatis, acuminatis, 4 millim. longis.

Equisetites Veronensis Zig. Mém. terr. jur. Alp. Ven. 1832, pag. 10; Bull. Soc. Geol. Franç. Series II. Vol. XI. pag. 291; Leonh. Bronn. Netr. Jahir. für Min. Geogn. Geol. 1854, pag. 54; Quart. Journ. Geol. Soc. Lond. Vol. X. n. ${ }^{\circ} 59$.

In calcarea scissili grisea vel lutescente ad montem Pernigotti in Valle Tanara et ad montem Alba prope Campo Fontana in Provincia Veronensi frequens, rarius occurrit in calcarea margacea grisea apud Rotzo in Val d'Assa Provinciae Vicentinae.

Questo Equisetite, di cui non mi fu possibile rinvenire la estremità superiore, sembra però che raggiungesse una considerevole lunghezza, la quale dal numero e dalle dimensioni dei frammenti raccolti si potrebbe calcolare per approssimazione non minore di un metro. Fra i varj esemplari che ne posseggo havvene uno della lunghezza di sessanta centimetri, la cui sommità è tronca, nè dà alcun indizio del termine della pianta.

La Tavola VI. rappresenta alcune porzioni del caule, e la parte inferiore congiunta al rizoma (Tav. VI. fig. 1.), che è grosso, bernoccoluto, repente, ramificato, e coperto di minutissime granulazioni.

Il caule è cilindrico, eguale, e senz’ alcun sensibile ingrossamento presso le articolazioni degl' internodj. Esso è segnato da finissime strie longiludinali, e da solchi paralleli corrispondenti a quelli delle guaine che vanno dileguandosi poco sotto le articolazioni. Sotto la base delle guaine stesse alcuni esemplari hanno dei segni circolari ora concavi, ora prominenti, che sembrano indicare il punto $d^{\prime}$ inserzione dei rami. Però non mi è avvenuto finora di rinvenire un esemplare ramificato. Il diametro del caule, prendendo una media fra tutti i saggi che ho potuto osservare, si può ritenere non minore di due centimetri, nè maggiore di due e mezzo. La lunghezza degl'internodj, e quindi la distanza fra $\mathrm{i}$ punti in cui hanno incominciamento le guaine, sta fra $\mathrm{i}$ quattro ed $\mathrm{i}$ cinque centimetri, e corrisponde cosi al doppio del diametro. Questa distanza diminuisce in quelle porzioni del caule che sono più da presso alla radice, come si osserva succedere negli Equiseti viventi. Le guaine sono molto accostate al caule, e dal punto della loro inser- 
zione fino alla base dei denti lunghe dagli ctto ai dieci millimetri. Sono alternamente segnate da solchi e da coste che si allargano in senso reciprocamente opposto, corrispondendo la parte più larga dei solchi allo spazio interposto fra i denti, e la più stretta alla base della guaina; mentre le coste che in quest'ultimo punto hanno una larghezza di circa un millimetro e mezzo, si stringono poscia gradatamente a guisa di cono, e vanno a formare il centro dell' estremità acuminata dei denti. Questi sono finissimi, subulati, lunghi quattro millimetri, un poco allargati alla base, alquanto discosti l'uno dall'altro, e separati da seni semicircolari. Dal numero di quelli che si possono discernere sulla porzione visibile dei saggi ho dedotto per approssimazione che possano essere all'incirca ventisei tutt' all' intorno.

La sola specie che a prima giunta sembra offrire qualche punto di rassomiglianza con questa, si è l'Equisetites Ungeri dell'Ettingshausen, trovato nelle marne liassiche a Hinterhol z presso Waidhofen e negli strati liasso-keuperiani di Reschitza nell' Ungheria. Ma qualora si esamini acuratamente quest'ultima, si scorgerà di leggieri quanto sia diversa dalla specie dell' oolite Veronese. L'Equisetites Ungeri ha il caule affatto liscio, le guaine lunghe due millimetri soltanto, i denti pochissimo allargati alla base e separati da seni quasi piani; mentre l'Equisetites Veronensis, come vedemmo, ha il caule striato, le guaine di una lunghezza giammai minore degli otto millimetri, $\mathrm{i}$ denti che partono da una base allargata che rapidamente si ristringe, e separati da seni semicircolari.

Fino dal 1852 ho dato il nome di Equisetites Veronensis a questa specie, perchè frequente nella calcarea bianco-giallognola del banco fitolitifero che ritengo coevo ai depositi di Scarborough, e che si osserva nel Veronese sul Monte Pernigotti, al Monte Alba, e sul Monte Raut. Dopo quell' epoca ne ritrovai qualche frammento nella calcaria grigia sottoposta al calcare ammonitico nella Valdassa al Sud di Rotzo nel Vicentino.

\section{Equisetites colmmnaris Sternb.}

E. caule erecto, simplici, laevi, cylindrico, diam. 4-10 centim.; internodiis inferioribus brevibus, superioribus 10 centim. longis et ultra; articulationibus incrassatis; vaginis erectis cauli adpressis, 2 centim. circiter longis, sulcatis, costatis; costis planis 2 millim. latis, apice lanceolatis; dentibus brevibus ovato-triangularibus in acumine caduco filiformi productis; sinubus obtuse lanceolatis $\mathbf{2}$ millim. inter se se discretis.

Equisetites columnaris. Stensi. Vers. Fl. Vorw. II. pag. 45. - Unger. Gen. el Spec. Plant. foss. pag. 56. - Eтtingsu. Monograph. Calam. foss. pag. 26 (exclus. pro parte Synon.).

FLORA FOSSILIS FORMATIONIS OOLITHICAE, - ACOTYLEDONES. 
Equisetum columnare. Brosg. Hist. Végét. foss. I. pag. 115. tab. 13. fig. 1. 2. 3. 4.

Oneylogonatum carbonarium. Koesig in Muncusos. Ou the Coal field af Brora. Trans. Geol. suc. Lond. Series II. vol. 2. pag. 293, tab. 32. fig. 1-6.

In arenaria carbonifera formationis oolithicae inferioris ad Haiburn-Wyke et ad Whitby in Comitatu York, nec non ad Brora in Comitatu Sutherlandiae (Young et Bird., Phillips, Murchison).

Gli esemplari di questo Equisetite figurati dai Signori Young e Bird, Murchison e Brongniart, e che provengono dai terreni oolitici dell'Inghilterra, presentano un caule eretto, semplice, liscio, cilindrico, avente un diametro che varia dai quattro ai dieci centimetri ed in cui la lunghezza degli internodii, che nella parte inferiore del caule è di circa tre centimetri, raggiunge i dieci centimctri nella parte superiore. Le articolazioni hanno un ingrossamento che in alcuni saggi è rilevante, ed aumenta ivi il diametro del caule di qualche millimetro.

Le guaine sono erette, appoggiate all' ingrossamento dell' articolazione, lunghe circa due centimetri, solcate longitudinalmente e munite di coste appianate larghe due millimetri e lanceolate all'apice ove le coste rapidamente convergono a formare la base dei denti. I denti di cui sono fornite sono brevi ovalo-lanceolato triangolari, e si prolungano in un'appendice filiforme acuminata, caduca; sono discosti l'uno dall' altro circa due millimetri e separati da seni ottusamente lanceolati.

Questa specie si è trovata negli strati inferiori della formazione oolitica ad Haiburı Wyke ed a Whitby nella Contea di York in Inghilterra ed a Brora nella Contea di Sutherland in Iscozia. Io qui pongo innanzi il parere che la specie trovata nei terreni oolitici d'Inghilterra e di Scozia sia diversa da quella rinvenuta nei terreni triassici dell'Alemagna ed a cui fu posto lo stesso nome. A dir vero non mi fu possibile di poter ottenere alcuno degli esemplari dei depositi Inglesi, ma posseggo all'incontro parecchi saggi della specie d'Alemagna provenienti dall' arenaria del Keuper dei contorni di Stuttgart.

In essi non si riscontra l' ingrossamento del caule alle articolazioni; le coste delle guaine, anzichè esser piane, sono scanalate verso l'apice, si attenuano assai prima e terminano molto più acuminate. I denti sono subulati e persistenti, ed i seni da cui sono separati sono semicircolari. Paragonati questi caratteri con quelli che si osservano sulle figure dell'Equisetites columnaris d'Inghilterra, quale è rappresentato nelle opere degli autori sopra citati, io sono indotto a ritener per ora i saggi d'Alemagna come spettanti ad altra specie. 


\section{$-67-$}

Avendo poi preso in accurato esame tutte le specie provenienti da altre formazioni che di recente furono riunite a questa (V. Ettingsh. Monogr. Calam. foss. loc. cit.), ebbi motivo di convincermi delle differenze che presentano confrontate colla specie di cui qui si tratta, e credo opportuno di farne qui cenno, onde render noti i motivi che mi persuasero ad escluderle dalla Sinonimia dell'Equisetites columnaris di Whitby, IIaiburnWyke e di Brora.

L'Equisetites cuspidatus, Strrno. non può essere riferito a questa specie perchè è munito di denti lineari angustato-acuminati, lunghi quanto le guaine e persistenti, ed ha inoltre il caule tutto segnato da solchi longitudinali indipendenti da quelli delle guaine.

Nell'Equisetites acutus, Sterss. sopra un caule del diametro di sette centimetri si veggono guaine lunghe appena un centimetro e mezzo, le cui solcature finiscono alla base della guaina e non si prolungano, come nell'E. columnaris, sulla parte superiore dell'internodio, chè anzi quest'ultimo è ivi segnato da uno o due solchi trasversali.

L'Equisetites Bromnii, Strena. (di cui sono sinonimi l'Equisetum arenaceum, Bronn. ed il Calamites arenaceus minor, JAEGER.), ha il margine degli articoli segnato da strie concentriche, le guaine brevi e tronche, e delle protuberanze tubercolari sui rami, caratteri che certo non l'agguagliano all'E. columnaris.

L'Equisetites Schoenleinii, Sternis. che è l'Equisetum platyodon, Brovg. è diverso dalla nostra specie pella larghezza assai maggiore dei denti delle guaine, e perchè secondo Sternberg ha il caule striato.

L'Equisetites Sinsheimicus, Sтеrкв. s'allontana dall'E. columnaris d'Inghilterra pella brevità e forma delle guaine, che molto somigliano invece a quelle dell'E. cuspidatus, col quale non esito d'identificarlo.

L'Equisetites areolatus, Sterrb. ha gli articoli segnati da linee trasversali che non esistono certo su quelli dell'E. columnaris. La mancanza poi di ogni traccia di guaine nell' esemplare figurato dallo Sternberg impedisce anche un lontano riferimento.

L'Equisetites conicus, Sterne. è pure assai diverso dalla specie oolitica, perchè ha i denti molto allargati alla base, senza appendice all'estremità e nettamente marcati da due nervature che si dipartono dall'apice del dente e scendono scostandosi gradatamente l'una dall'altra fino alla base della guaina.

L'Equisetites elongatus, Sтвrns. che ha le lacinie delle guaine lanceolate, allungate, marginate, lunghe oltre due centimetri e larghe alla base cinque millimetri è senza dubbio specie affatto differente da questa e da tutte le altre.

Finalmente l'Equisetites austriacus dell' Unger, che ha il caule molto striato e le guaine moltifide colle lacinie lineari cuspidate, sarebbe anch' esso totalmente diverso dall'Equisetites columnaris dell'oolite. 


\section{$-68$}

Tracciate cosi brevemente le differenze che mi è sembrato scorgere fra le sopraccennate specie e l'Equisetites columnaris di Haiburn Wyke, di Whitby e di Brora, rimane ad osservarsi come la riunione di tulte queste diverse forme nella sinonimia dell'Equisetites columnaris avvalori il sospelto, che solto questa denominazione siano state finor comprese piu specie diverse. Perciò tengo ferma credenza che l'accurato studio dei resti fossili attribuiti a questa specie tanto in Inghilterra che in Alemagna, farà vie meglio rilevare le differenze specifiche sussistenti fra i saggi che provengono dai terreni oolitici d'Inghilterra e quelli osservati nelle arenarie Keuperiche della Germania.

\section{Classis IV. FILICES.}

Le Felci costituiscono una parte assai rilevante nella Flora fossile delle diverse formazioni geologiche, specialmente dopo le scoperte fatte negli ultimi anni che ne portarono il numero al quarto di tutte le piante fossili conosciute.

Quando il Brongniart nel 1828 imprendeva la sua grande opera sulle piante fossili (1), le Felci sommavano a sole 178 specie. Nel Systema Filicum Fossilium di Göppert stanpato nel 1836, le vediamo giungere a 253. Tredici anni dopo, lo stesso Brongniart, nella sua memoria intitolata Pèriodes de Végétation et Flores des divers terrains (2), ne porta il numero a 482. Bronn nell'Index Paleontologicus che vide la luce in quel torno ne annovera 516 specie, e l'Unger nel Genera et Species Plantarum fossilium stampato nel 1850 le aumenta fino a $5 \% 2$, alle quali aggiungendo tutte quelle che si scopersero di poi si può concludere, senza tema di andare errati, che presentemente il novero delle felci fossili conosciute sia forse maggiore, ma certo non minore delle 600 specie.

Fra queste, due terzi all'incirca spettano alle formazioni geologiche più antiche, cioè ai terreni di transizione ed a quelli della formazione carbonifera. Il rimanente è distribuito con ineguale misura nelle epoche geologiche successive. Poichè, mentre si fanno repentinamente scarse nei terreni Permiani, ne aumenta gradatamente il numero nei terreni Triassici e Liassici, si fa piuttosto rilevante nella serie Oolitica, poi torna a decrescere nell' argilla di Weald e procede minorando nei terreni Cretacei e Terziarj. Giova però osservare come nella formazione Carbonifera ed in quella dell' Oolite, quantunque

(1) Brongniart. Histoire des Végétaux fossiles. Paris 1828-36.

(2) Annales des Sciences Naturelles. Vol. XI. a. 1849. 
separate dalle potenti stratificazioni degli altri terreni interposti, le Felci si trovino in eguale proporzione numerica in confronto delle altre piante e costituiscano, tanto nell'una che nell' altra formazione, circa due quinti delle rispettive Flore.

Questa eguale proporzione relativa delle Felci rispetto alle Flore di due epoche geologiche, poste a si grande distanza fra di loro nell' ordine cronologico delle formazioni, è un fatto che merita di essere avvertito, ma a cui dobbiamo guardarci dall' attaccare troppo grande importanza, imperciocchè future scoperte potrebbero per avventura alterarne di gran lunga gli estremi.

'Trent' anni fa si conoscevano appena trenta specie di Felci proprie della formazione Oolitica. Nel 1849 Brongniart (1) ne portò il numero a quarantaquattro e l'Unger nel 1850 ne descrisse cinquanta. Negli ultimi quattordici anni mercè le scoperte di Göppert, di Pomel, di Bunbury e di Leckenby si aumentarono d'altre diecinove specie, alle quali aggiungendo ventisei nuove specie trovate nelle Alpi venete, il numero attuale delle Felci conosciute nella Flora dell' Oolite si troverà quasi raddoppiato, giungendo ora a novantacinque specie.

Come è noto le Felci fossili furono distribuite in varii gruppi od ordini fondati principalmente sulla disposizione della nervatura. Questi gruppi che servono a distinguerle e coordinarle devonsi considerare come provvisorii al paro delle denominazioni generiche di quelle forme che finora non ci hanno svelato alcun indizio della loro fruttificazione.

Alcune di queste divisioni artificiali, nei caratteri loro assegnati, non corrispondono colla esattezza che sarebbe desiderabile, con quelli dei generi che vi sono compresi; c per tal motivo ho creduto opportuno introdurvi qualche riforma, ed aggiungerne taluna di nuova, per quelle Felci clie non potevano assolutamente prender posto negli ordini già stabiliti.

Perciò ho aggiunto l'ordine delle Pachitteridee per collocarvi il genere Pachypteris del Brongniart. Questo genere distinto per avere le pinnule percorse da un solo nervo mediano non s'attaglia per questo e per altri caratteri ad alcuno degli ordini esistenti, e la sua nervatura congiunta alla consistenza coriacea della fronda aveva indotto il Prof. Unger a porlo con qualche dubbio fra le Cicadeacee. Ma la forma bipinnata della fronda non permette di acconsentire a tale avvicinamento.

Separai dalle Neurotteridee le Felci a nervature eguali disposte a ventaglio o flabellate, cioè i generi Cyclopteris, Odontopteris, Dichopteris, concentrandoli tutti nel nuovo ordine delle Ciclotteridee.

Formai un altro ordine che chiamai delle Dittioterridee per tutti $\mathrm{i}$ generi a nervature reticolate che colle loro anastomosi formano delle maglie od areole più o meno

(1) Brongniart. Periodes de Végétation et Flores des divers terrains. Ann. Sciences Nat. Vol. XI. 
allungate, sia che queste si estendano per tutta la pinnula, sia che rimangano limitate ad alcun punto di essa, ed in questo annoverai i generi Camptopteris, Phlebopteris, Sagenopteris ed il genere Protorhipis di Andrae.

Finalmente pel nuovo genere Stachypteris del Pomel creai l'ordine delle Stachitteridee, destinandolo per le felci a fruttificazione disposta in forma di spica ed inserita sulle estremità delle rachidi.

Mi valsi poi degli ordini di già ammessi delle Sfenotterridee, delle Neurotteridee, delle Pecotteridee, delle Gleicheniacee e delle Daneacee per disporvi gli altri generi trovati in questa formazione, secondo fecero altri autori, però con qualche modificazione diretta ad agevolarne il riferimento.

Esclusi dalla sinonimia della Pachypteris lanceolata e della Pachypteris ovata del Brongniart, la Sphenopteris lanceolata e la Neuropteris laevigata di Phillips, giacche la nervatura flabellata di quest' ultime, quale si scorge nelle figure del Phillips, sta in contraddizione col carattere assegnato al genere, e mostra ad evidenza che sono due piante diverse da quelle descritte nell'Histoire des Végétaux fossiles del Brongniart.

Di questa disposizione dei nervi ebbi conferma dallo stesso Phillips; perciò collocai queste due forme nel mio genere Dichopteris col quale presentano non poche analogie tanto per la nervatura, che per l'aspetto generale e la forma delle pinnule (1).

Tenni le Hymenophyllites di Göppert separate dalle Sphenopteris del Brongniart: tanto più che anche quest' ultimo ammise tale separazione nel suo Tableau des genres stampato nel 1850.

Nella stessa opera il Brongniart esponeva i caratteri del nuovo genere Phyllopteris destinandolo per quella Felce che aveva descritta parecchi anni prima nella Histoire des Végétaux fossiles sotto il nome di Glossopteris Phillipsii, e che Phillips aveva figurata nella Geology of the Yorkshire coast col nome di Pecopteris longifolia e paucifolia.

La nervatura di questa Felce è evidentemente pinnata coi nervetti secondarii molto obbliqui e senza traccia alcuna di reticolazione. Dal che ne consegue che essa fu annoverata a torio dagli autori ora fra le Glossopteris ed ora fra le Sagenopteris cosi confondendola con altra specie a nervature reticolate. Stimai quindi opportuno conservare il genere Phyllopteris proposto dal Brongniart e rettificare in conseguenza di cio la sinonimia della specie.

Serbai pure i generi Polypodites di Göppert e Phlebopteris del Brongniart annoverando nel primo le sole specie a nervatura pinnata, e nel secondo quelle in cui i nervetti secondarii si congiungono in areole reticolate lungo il nervo principale, e quindi

(1) Vedi Zigno Monografia del genere Dichopteris nel volume XII delle Mem. dell'I. R. Istituto Veneto di Scienze Lettere ed Arti. 


\section{$-71$}

posi la Phlebopteris Woodwardii di Leckenby nel genere Polypodites perchè si mostra mancante di questo carattere.

Egualmente collocai nel genere Gleichenites la Pecopteris Desnoyersii del Brongniart, non porgendo essa alcun indizio che possa raffermare la sua appartenenza alle Pecotteridi ed avvicinandosi piuttosto alle Gleicheniacee per la forma delle pinnule.

Non devo ommettere di qui ricordare le due singolari forme vegetali trovate nell' $0_{0}$ lite di Cloughton Wyke in Inghilterra, descritte e figurate dai Signori Lindley e Hutton nella Fossil Flora of Great Brittain, Tom. III. Tav. 170 sotto il nome l'una di Tympanophora simplex, Fig. A, l'altra di T. racemosa, Iig. B.

Forme consimili si rinvennero in Francia dal Pomel nei terreni coralliani di Seyssel e dal Moreau in quelli di S. Michel. Il Pomel nella sua memoria sulla flora giurassica della Francia che presentò alla riunione dei naturalisti e medici Tedeschi ch' ebbe luogo in Aquisgrana nel Settembre del 1847, collocò tutte queste forme fra le Alghe, vedendo in esse qualche analogia col Fucoides turbinatus e col F. discophorus del Brongniart. Se non che qualche anno dopo il signor Bean che si è occupato con molta diligenza delle piante fossili di Scarborough esternò la opinione che la Tympanophora racemosa altro non fosse che lo stato fertile di qualche fronda di Felce e precisamente della Pecopteris Murrayana. Giò venne recentemente confermato dal Leckenby che diede la fignra di un saggio della Pecopteris Murrayana, in cui le pinne superiori presentano appunto l'aspetto della $T$. racemosa (1). Per ciò poi che risguarda la Tympanophora simplex il Leckenby sospetta che sia lo stato fertile della Pecopteris lobifolia L. II. Io pure trovai nell' Oolite del Veronese una fronda le cui pinne perfettamente s'altagliano colle figure della Tympanophora racemosa, ma non avendo ancor poluto rinvenirne un saggio in cui sia bene spiegata la forma delle pinnule nelle pinne sterili, non credo che per ora si possa con sicurezza identificarla colla Pecopteris Murrayana. Per tal motivo ho provvisoriamente descritta questa forma sotto il nome di Hymenophyllites tympanophoraeformis, fino a che migliori esemplari giungano a chiarirne la vera natura.

Lasciai per ora il genere Dichropteris di Pomel nell' ordine delle Sfenotteridee, ove fu temporaneamente collocato dallo stesso Pomel, quantunque la descrizione ch' egli ne porge non avvalori gran fatto questa classificazione, imperciocchè nessuno dei saggi fin qui raccolti palesa in modo abbastanza distinto quale sia la reale distribuzione dei nervi.

Con eguale incertezza collocai nello stesso ordine il genere Loxopteris del medesimo autore per le ragioni che indichero a suo luogo.

In quanto poi alle specie trovate nelle Alpi Venete noi le veggiamo abbondare per lungo tratto nelle calcaree grigie e giallognole soltoposte agli strati dell'Oolite media.

(1) Leckenby. Quart. Journ. Soc. Geol. London. Vol. XX Feb. 1864. 
A Rotzo, a Mezzaselva, nella Valdassa, a Grespadoro, Durlo e Marana nella Provincia di Vicenza; ai Pernigotti, a Bienterle, a Scandolara, nel Monte Alba, sul M. Raut, nella Valle Zuliani, ed in quelle di Salaorno e di Squaranto nella Provincia Veronese, ovunque palesano con numerosi esemplari la loro presenza.

Lo studio di così estesa e ferace miniera trasse fin qui in luce trentadue specie di Felci. Sei delle quali già note e comuni ai depositi oolitici d'altri paesi, e ventisei affatto nuove, cioè: 1 Hymenophyllites; 1 Gyclopteris; 1 Odontopteris; 5 Dichopteris; 4 Cycadopteris; 6 Sagenopteris; 1 Protorhipis; 1 Gleichenites; 1 Laccopteris; 3 TaeMiopteris; ed il nuovo genere Marzaria con due specie.

Quindi pel numero delle specie di questa famiglia la Flora Oolitica delle nostre montagne vien subito dopo quella dell' Inghilterra, che ne annovera quarantanove, ed è assai più numerosa di quella della Francia ove se ne trovarono diciasette, e di quella dell'Alemagna che finora ne comprende soltanto sette.

Da questi confronti è agevole riconoscere come le scoperte fatte nell'Alpi Venete abbiano accresciuto di oltre un quarto il numero delle Felci osservate nelle stratificazioni della formazione Oolitica.

L'illustre Brongniart nella più volte citata sua Memoria intitolata Périodes des Végétation et Flores des divers terrains parlando dei caratteri generali di cui è improntata la Flora dell' Oolite, accenna, per ciò che risguarda le Felci, alla scarsezza di quelle che sono fornite di una nervatura reticolata, mentre nella precedente Flora del Lias si erano invece mostrate più numerose. Ed è rimarchevole come ad onta delle svariate forme scoperte di poi che portarono il numero delle specie al doppio di quelle che si conoscevano nel 1850, questo carattere tuttora distingue la Flora dell'Oolite, sebbene varie specie di Sagenopteris ed una Protorhipis siano venute ad arricchirla.

E per vero se una dozzina di Felci a nervatura reticolata si osserva fra le cinquanta specie della formazione Liassica, dieci sole ne svela la Flora dell'Oolite sopra un numero complessivo di 94 specie, di guisa che rimangono tuttavia inalterate le proporzioni numeriche relative indicate quindici anni or sono dal Brongniart.

Che se poi ci facciamo a considerare la loro distribuzione geografica, noi le abbiamo vedute assai rare nei terreni oolitici della Germania, farsi alquanto più numerose in quelli della Francia, aumentare assai più nei depositi del Veneto, e palesarci il maggior numero di specie negli strati oolitici dell' Inghilterra.

Ai quali depositi ora si aggiungono gli strati giuresi di la Dorche in Svizzera, ove il Prof. Heer trovò dei saggi della mia Cycadopteris Brauniana, da lui considerata quale un eccellente tipo caratteristico per riconoscere la Flora del Giura. Non tutti però questi depositi appartengono allo stesso orizzonte geologico. Quelli di Scarborongh e di Stones- 


\section{$-73$}

field nell' Inghilterra giacciono intercalati a stratificazioni contenenti i fossili proprj della serie oolitica inferiore.

Quelli delle Alpi Venete dimostrai fino dal 1850 appartenere alla stessa serie, ed il ritrovamento del Polypodites undans Göpp, del Polypodites crenifolius Göpr, della Phlebopteris Polypodioides Brong, della Phlebopteris contigua Lind, Hut e della Sagenopteris cuneata Morris, specie comuni ai depositi d'Inghilterra confermò pienamente questa classificazione.

A questo stesso gruppo dell' Oolite inferiore mostrano di appartenere gli strati giuresi con piante fossili della Slesia e dell'Austria, e quelli di Namer's nella Francia, mentre quelli di Verdun, S. Michel, del Bugey, di Alençon, di Chateauroux e Chatillon sur Seine e quelli pure di Solenhofen, Schnaitheim e Nussplingen nell'Alemagna sono finora riferiti dalla maggior parte dei geologi al terreno coralliano, ossia al piano superiore della serie oolitica media.

\section{Ordo VIII. Pachypterideae.}

Frons pinnata vel bipinnata. Pinnae vel pinnulae uninerviae. Zıко, Enum. Filic. foss. formationis Oolithicae. pag. 19.

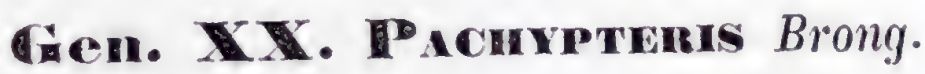

Frons pinnata vel bipinnata. Pinnae vel pinnulae integrae, coriaceae, basi angustatae, in rachide decurrentes, uninerviae, nervo plus minusve notato.

Brong. Prodrom. pag. 50 ; Hist. Végét. foss. I. pag. 166 ; Tabl. Gen. plant. foss. pag. 34. Stenrs. Vers. Fl. Vorw. II. pag. 55. - Göpr. Syst. Filic. foss. pag. 179, et suppl. pag. 378. UNG. Synops. pag. 165; Gen. et Spec. plant. foss. pag. 307.

\section{Pachypteris Ianceolata Brong.}

P. fronde pinnata vel bipinnata? rachide plana submarginata, pinnis pinnulisve sub angulo acuto patentibus, oppositis, lanceolatis, obtusis, sursum retuse unidentatis, basi angustatis, decurrentibus; nervo medio tenuissimo.

pachypteris lanceolata. Brosg. Prodrom. pag. 50 ; Hist. Végét. foss. I. pag. 167, tab. 45, fig. 1. a, b, (exclus. synon. Sphenopteridis lanceolatae. Puncles. Geol. York. tab. 10, fig. 6). - Presz. in Stenrb. Vers. Fl. Vorw. II. pag. 55 (exclus. synon. Palulirs.) - Göpr. Syst. Filic. foss. pag. 179 


\section{$-77-$}

ralmente minore e sono alquanto compresse, locclie forse è dovuto alla fossilizzazione. Le pinne, che gli autori indicarono come assai patenti, a parer mio forse non lo erano quanto si è creduto, avvegnachè bene osservando la figura del Brongniart, si scorgera di leggieri come, verso la base, la rachide secondaria si mostri spezzata, ed alcun poco disgiunta dalla rachide principale, per cui non puossi affermare con sicurezza qual fosse il suo modo d'inserzione e la vera sua direzione. Nella citata figura si vede pure mancare sul saggio tutta l'estremità della pinna. Però dal pezzo esistente e lungo tre centimetri si può arguire, che questo raggiungesse appena il terzo della totale lunghezza. poichè la rachide secondaria corre quasi eguale per tutto quel tratto, nè dà alcun indizio di rapido assotligliamento, chè anzi il diametro, piuttosto rilevante in confronto delle altre specie di egual portamento, farebbe sospettare dimensioni assai maggiori.

La pinna è guernita di pinnule distanti l'una dall'altra dai due ai tre ed anche quattro millimetri, lunghe dai dieci ai quindici e larghe all'incirca quattro, sessili, alterne, lanceolate, acute e pinnatifide a segmenti oblunghi, verso la base un po'ristretti e separati da seni ottusi, verso l'apice più espansi, ottusi, obliquamente tronchi e muniti di tre o quattro denti. Il segmento terminale è lanceolato, fornito nella estremità di due o tre denti per parte, obliqui e decrescenti verso l'apice del segmento che termina in punta.

Un sottil nervo principale scorre lunghesso la pinnula, e nel segmento terminale si risolve in altrettanti nervilli quanti sono $\mathrm{i}$ denticelli in cui è partito quest'ultimo. Dal nervo principale escono in forma pinnata i nervicciuoli secondarii per lo più geminati nella parte inferiore e forcuti superiormente, che percorrono gli altri segmenti della pinnula, meno quelli della base ove i nervetti non escono dal rervo primario, ma scorrono paralleli per breve tratto dal punto d'inserzione sulla rachide secondaria e poi s'immettono biforcandosi nei due prini segmenti laterali posti alla base della pinnula.

L'illustre Brongniart accenna come per la forma delle pinnule e per l'apparenza consistente e liscia della fronda, questa specie a primo aspetto si avvicini ad alcune specie di Davallie ed in particolare alla Davallia pyxidata $C_{A}$. ed alla Davallia epiphylla Sw. o Wibelia multifiala Bersn. Però fa in pari tempo rilevare come la specie fossile sia bastantemente da quelle distinta pelle profonde divisioni dei lobi o segmenti in cui è partita cadauna pinnula, al che troverei d'aggiungere anche la forma dei segmenti slessi e la base più allargata della pinnula.

Questo frammento fu trovato nei terreni oolitici di Scarborough lungo la costa della Contea di York dal chiarissimo sig. Williamson noto per le molte sue scoperte di piante fossili in que' terreni giurassici. I signori Young e Bird, Phillips, Lindley e IIutton non 


\section{- $79-$}

L'esemplare presente trovato nelle roccie della formazione oolitica di Scarborough ci porge una specie decisamente diversa dalle altre, sebbene da taluno si abbia voluto vedere in essa un frammento della Sphenopteris tenuifolia Brong. del terreno carbonifero di Francia. Il semplice confronto tra la figura di quest' ultima specie data dal Brongniart (Hist. Végét. foss. tab. 48, fig. 1.) e quella della Sphenopteris arguta di Lindl. e Hutton loc. cit., basterà a chiarire l'erroneità di un tale avvicinamento.

\section{Sphenopteris eysteoides Lindl. e Hutt.}

Sph. fronde bipinnata, pinnis alternis remotiusculis, patentibus; pinnulis subpetiolatis alternis irregularibus, inferioribus profunde pinnatifidis vel inciso lobatis 2-5-4 fidis, summis simplicibus lanceolato oblongis cuneatis, apice obtusis; rachide rigidula; nervis obsoletis.

Sphenopteris cysteoides. Linde. et Hutr. Foss. Flor. Gr. Brit. Vol. III. Lab. 176, A. - Morris. Catal. Brit. foss. pag. 21. - Zigno, Enum. Filic. foss, form. Oolith. pag. 20.

In schisto calcarifero seriei oolithicae inferioris ad Stonesfield Angliae invenit Glarissimus Doctor Buckland.

La fronda, quale ce la figurano i signori Lindley e Ilutton, si palesa bipinnata colla rachide di apparenza un po' rigida e lievemente ingrossata al punto d'inserzione delle pinne. Quest'ultime, lunghe poco più di dodici millimetri, sono alterne, alquanto distanti l'una dall'altra er assai patenti. Le pinnule che sono pure alterne e si attenuano tutte quasi in forma di picciuolo verso la base, si mostrano di forma piuttosto irregolare; le inferiori essendo talvolta pinnatifide, ma più di spesso profondamente divise in due, tre e persin quattro lobi o lacinie, mentre le superiori sono semplici o tutt'al più bifide coi segmenti angustissimi cuneato-lanceolati ed ottusi all'apice. Le rachidi secondarie sono filiformi, e niun indizio dei nervetti puossi scorgere sulla figura che ne porgono i signori Lindley e Ilutton.

Questa specie mi sembra a torto riunita dai signori Göppert ed Unger alla Sphenopteris multifida L. II. ed alla Sphenopteris tenella Brong., che oltre a ciò sarebbero, a parer mio, diverse anche fra di loro.

Differisce principalmente dalla prima pei lobi o segmenti lanceolato-allungati, cuneiformi alla base ed ottusi all'apice e pella maggior consistenza della rachide, mentre nella Sphenopleris multifida $\mathbf{L}$. II. Ie pinnule sono in generale più regolarmente par- 
tite ed a lobi lineari lanceolati acuti colla base assai meno attenuata, e la rachide si mostra esilissima e flessuosa.

All'incontro la Sphenopteris tenella Rrowg. colle pinnule ovato-oblunghe profondamente pinnatifide a segmenti regolari non attenuati verso la base, e la cui disposizione, al dire del Brongniart, rammenterebbe quella delle Pecopteris, non mi sembra possa essere riunita nè alla $S p h$. cysteoides, nè alla $S p h$. multifida. Oltre a ciò tanto la $S p h$. tenella Brong., quanto la Sph. multifida L. II. si rinvennero nel terreno carbonifero, mentre il saggio descritto dagli autori della Flora fossile della Gran Brettagna fu rinvenuto dall'illustre dott. Buckland nello schisto calcarifero di Stonesfield, che appartiene alla serie oolitica inferiore.

Anche questa diversa provenienza da formazioni collocate a si grande distanza l' una dall'altra nell' ordine cronologico dei terreni avvalora la proposta separazione della Sph. cysteoides L. H., e giustifica maggiormente l'aver io escluso dalla sua sinonimia le due specie che Göppert ed Unger avevano ad essa riunite.

\section{Sphenopteris athyrioides Presl.}

S. fronde bi-tripinnata, pinnis oblongis, pinnulis alternis patentissimis sessilibus, linearibus, obtusis, profunde pinnatifidis, basi non decurrentibus; laciniis ovatis, obtusis, rachim marginantibus, infimis pinnatifidis, superioribus adnatis, terminalibus confluentibus, lacinulis vel lobis acute tri-bidentatis, approximatis; nervulis tenuissimis, obliquis, bi-tri-furcatis.

Sphenopteris athyrioides. Prest, in Sterna. Vers. Flor. Vorw. II. p. 431. - Morris. Calal. Brit. foss. pag. 21. - Ung. Synops. pag. 68; Gen. et Spec. plant. foss. pag. 127. - Zigro, Enum. Filic. foss. form. Oulith. pag. 20.

Pecopteris athyrioides. Brosa. Hist. Végét. foss. I. pag. 360, tab. 125, fig. 3.

Coniopteris athyrioides. Brong. Tabl. Gen. Végét. foss. pag. 26, 105.

In formatione oolitica ad Saltwick prope Whitby Angliae.

Il parallelismo delle due pinne che si osservano sul saggio posseduto dalla Società filosofica di York, descritto e delineato dal Brongniart, gli fece giustamente sospettare che tutte due appartenessero alla stessa fronda, e questa fosse quindi tripinnata.

Queste pinne ci si palesano oblunghe e pinnate, colle pinnule lineari molto patenti, sessili, alterne, lunghe circa ventisette millimetri e larghe otto, profondamente pinnatifide colla base non decorrente e coll' estremità ottusa. Le lacinie ovate, ottuse, formanti ala 


\section{$-74$}

(exclus. synon. Pnilurs.). - Unger. Gen. et Spec. plant. foss, pag. 307 (exclus, synon. Puiluirs.). - Zigno, Enum. Filic. foss. form. Oolith. pag. 19.

In stratis inferioribus formationis oolithicae ad Whitby in comitan York, Angliae.

Il saggio figurato dal Brongniart ci presenta una fronda semplicemente pinnata, ma non esclude il dubbio che possa invece rappresentare una pinna di una fronda bipinnata. La rachide che la percorre è appianata e fiancheggiata longitudinalmente da una specie di margine. Essa ha un diametro verso la base di due millimetri compreso il margine, e va lentamente assottigliandosi verso la cima. Le pinne, o pinnule che sieno, sorgono ad angolo acuto dalla rachide, e poscia si scostano facendosi alquanto patenti, però conservando una generale obliquità. Sono opposte, lanceolate, ottuse, superiormente retuso-unidentate, attenuate verso la base e decorrenti. La loro consistenza è coriacea, e sono percorse da un nervo mediano piuttosto sottile, che si prolunga fino all'apice delle pinnule. La lunghezza di quest' ultime è di circa sedici millimetri, e misurano poco più di quattro millimetri nella loro maggiore larghezza.

Fu trovata nelle stratificazioni inferiori della formazione oolitica a Whitby nella Contea di York nell' Inghilterra.

\section{Pachypteris ovata Brong.}

P. fronde bipinnata; rachide crassa, plana, marginata; pinnis linearibus profunde pinnatifidis, alternis, oblique patentibus, remotis; pinnulis obovato ellipticis, basi angustatis, superioribus basi latioribus, ovato oblongis, obtusis, terminali oblonga, serrata, apice truncala, basi acuta; nervo ante apicem evanescente.

Pachypteris ovata. Brong. Prodrom. pag. 50 ; Hist. Végét. foss. I. pag. 168, tab. 45, fig. 2, a, b, (exclus. synon. Neuropteridis laevigatae Pullups.). - Prest. in Stenvi. Vers. Fl. Vorw. II. pag. 55 (exclus. synon. Pmllips.). - Göpr. Syst. Filic. foss. pag. 179 (exclus, synon. Pmillips.). - Usg. Gen. et spec. plant. foss, pag. 308 (exclus, synon. Phillips.). - Zigro, Enum. Filic. foss. form. Oolith. pag. 19.

La fronda in questa specie si palesa bipinnata e munita di una rachide primaria piuttosto grossa, appianata e marginata, avente un diametro di tre millimetri. Le pinne sono profondamente pinnatifide, lineari, alterne, oblique e patenti. Sono discoste l'una 


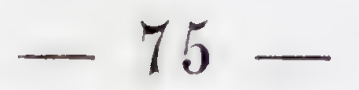

dall'altra oltre due centimetri, ed hanno una larghezza di dodici millimetri all'incirca. Le rachidi secondarie che le percorrono sono pure appianate e munite di un' espansione membranacea continua colla base delle pinnule. Le pinnule o lacinie sono obovato-ellittiche, un po' rotondate all'apice ed attenuate alla base, larghe poco più di tre millimetri e lunghe dai sette agli otto. Le superiori passano alla forma ovato-oblunga, ottusa all'apice e colla base più larga. La pinnula terminale si protende in forma oblunga più delle laterali. Essa è tronca all' estremità, un po' seghettata, ed acuminata ver'so la base. Il nervo mediano di cadauna pinnula s'assottiglia e svanisce verso l'apice.

Questa specie fu trovata colla precedente nella stessa formazione e nel medesimo $\log 0$.

Si vedrà più innanzi, ove tratto del genere Dichopteris, quali furono i motivi che m'indussero ad escludere dalla sinonimia della Pachypteris lanceolata e della Pachypteris ovata del Brongniart, la Sphenopteris lanceolata e la Neuropteris laevigata di Pundrrs. Nella mia Monografia del genere Dichopteris (Mem. dell'I. R. Istit. Veneto, Vol. XII, 1865) lo già fatto conoscere i caratteri che distinguono quest'ultime dalle Pachypteris del Brongniart, e che mi parvero sufficienti a giustificare la loro collocazione in un altro genere.

\section{Pachypteris Brongniartiana Zign.}

P. fronde.... pinnis in rachide decurrentibus; pinnulis coriaceis, obovatis, obtusis, basi non constrictis.

In formatione oolithica apud Chatillon sur Seine in Gallia (Brosg. Tabl. Gen. végét. foss. pag. 34).

$$
\text { Species non descripta. }
$$

\section{Wachypteris microphylla Brong.}

In formatione oolithica (coral rag.) ad Verdun Galliae (Brong. Tabl. Gen. végét. foss. pag. 3/4 et pag. 105). 


\section{Ordo IX. Sphenopterideae.}

\section{Gen. XXT. Spmevopinens Brong.}

Frons bi-tripinnata vel bi-tripinnatifida, pinnulis lobatis, rarius subintegris basi cuncatis, lobis inferioribus majoribus dentatis vel sublobalis. Nervi pinnati; nervo primario subdistincto excurrente subflexuoso, nervis secundariis laxis oblique adscendentibus simplicibus vel dichotomis, ramis in singulis lobis bis, raro ter, furcatis. Fructificatio punctiformis vel marginalis.

Brong. Prodrom. pag. 50; Hist. Vég. foss. I. pag. 169 ; Tabl. des Genres des Végét. foss. pag. 20. - Sтеrвв. Vers. Flor. Vorw. II. pag. 55. - Göpp. Galt. foss. Pf. I. pag. 67. - Uxсrn. Synops. pag. $59 ;$ Gen. et Spec. plant. foss. pag. 107.

\section{Sphenopteris dentieulata Brong.}

Sph. fronde bipinnata, pinnis patentissimis, pinnulis alternis, sessilibus, lanceolatis, acutis, pinnatifidis; laciniis oblongis, obtusis, apice tridentatis vel quadridentatis, sinubus obtusis; rachide primaria tereti, secundaria compressa; nervis pinnatis; nervulis in laciniis inferioribus geminatis, apice furcatis.

Sphenopteris denticulata. Brosg. Prodrom. pag. 50 ; Hist. végét foss. I. pag. 188, lab. 56 , fig. 1. a, b. Tabl. Gen. Végét. foss. pag. 105. - Sterinв. Vers. Flor. Vorw. II. pag. 61. - MonRus. Catal. Brit. foss. pag. 22. - Uxg. Synops. pag. 60; Gen. et spec. plant. foss. pag. 108. Zigro, Enum. Filic. foss. form. Oolith. pag. 20.

Cheilanthites denticulatus. Görp. Syst. fil. foss. pag. 232.

In formatione oolithica prope Scarborough Angliae invenit clarissimus Williamson.

Non è a mia cognizione che siensi trovati saggi più compleli del frammento sul quale Brongniart fondava questa specie. Perciò si rimane ancora nell' incertezza circa alla forma generale e precisa suddivisione della fronda. Tuttavia dal saggio, di cui Brongniart ci diede la figura, possiamo arguire che fosse certo bipinnata e for's' anco tripinnata. La rachide primaria di forma cilindroide e colla superficie piuttosto liscia, misura dai due ai tre millimetri di diametro. Le rachidi secondarie hanno un diametro natu- 


\section{$-78$}

famno menzione di questa specie. Brongniart la descrisse e ne diede la figura nella sua classica Histoire des Végétaux fossiles. Dopo di lui Sternbergog, Göppert ed Unger la riportarono e descrissero nelle loro opere. Il secondo però la separò dal genere Sphenopteris ponendola con altre specie nel suo genere Gheilanthites. Non essendone ancor nota la fruttificazione, ritenni coll' Unger questa specie nel genere cui fu riferita dal Brongniart.

\section{Sphenopteris arguta Lind. Hutt.}

Sph. fronde bipinnata; pinnulis subrhombeis, aculis, versus apicem decrescentibus, profunde pinnatifidis; laciniis trijugis, cuncatis, obtusiusculis, infimis tridentatis, mediis summisque bidentatis; nervis pinnalis, furcalis; rachide prominula, teretiuscula.

Sphenopteris arguta. Lind. Hutr. Foss. Fl. of G. Brit. Vol. III. Lab. 168. - Stennd. Vers. Flor. Vorw. 1I. pag. 130. - Monns. Catal. Brit. foss. pag. 21. - Ung. Synops. pag. 62; Gen. et Spec. plant. foss. pag. 113. - Zigno, Enum. Filic. foss. form. Oolith. pag. 20.

In stratis formationis oolithicae ad Scarborough Angliae invenit Cl. Williamson.

I signori Lindley e Hutton autori della Flora fossile della Gran Brettagna ebbero il primo saggio di questa specie dal chiarissimo sig. Williamson giuniore, accompagnato da alcune osservazioni che riportarono letteralmente nell' opera suddetta. Questa specie ci presenta una fronda di piccola dimensione, elegantemente acuminata, colle pinne pinnatifide, se si voglia considerare nell' esemplare in discorso l' estremità dell'intera fronda, e non piuttosto una delle pinne di una fronda assai maggiore, come dubitano alcuni autori assegnandole il carattere della fronda bipinnata, e chiamando pinnule quelle che nel primo caso dovrebbero dirsi pinne. Quest'ultime sono lunghe circa otto millimetri e larghe quattro; hanno forma romboidale attenuata alla base ed ottusa all'apice, e si dividono profondamente in lobi che decrescono di numero in quelle più vicine all' estremità. Le inferiori quindi appariscono pinnatifide colle lacinie pure partite in lobi piuttosto ottusi, di cui tre si scorgono formar l'apice delle lacinie più vicine alla rachide, e due soli quello di tutte le altre, toltane l'ultima terminale che è ottusamente tridentata. La nervatura è pinnata, e i nervetti laterali che scorrono semplici per alcun tratto nel mezzo dei segmenti delle pinnule si fanno forcuti partendosi nel senso dei lobi terminali. La rachide è alquanto prominente, liscia e senza alcuna traccia di coste od angoli . 
alla sottil rachide son pinnatifide nella parte inferiore della pinna, e verso la cima confluenti. I lobi in cui sono obliquamente partite, sono avvicinati e forniti nell' estremità di due o tre denti mediocremente acuti, ed i nervicciuoli che li percorrono si biforcano in guisa, che ogni ramificazione corrisponde ad una delle addentellature dei lobi.

Non si saprebbe spiegare come l'Unger abbia citato a proposito di questa specie il Göppert, che invece non ne fa alcuna menzione nè nel Sỵstema Filicum fossilium, nè manco nell'Opera intitolata Gattungen der fossilen P'flanzen. Fu all'incontro il Presl che pel primo collocò nel genere Sphenopteris questa specie, che il Brongniart aveva descritta sotto il nome di Pecopteris athyrioides, ponendola però nella sua Sezione delle Pecopteridi sfenotteroidi. Nell'opera dello Sternberg, Vers. Flor. Vorw. loc. cit., fu ritenuta fra le Sphenopteris, e così pure in quelle del. Morris e dell'Unger.

Posteriormente il Brongniart nel suo Tableau des Genres des Végétaux fossiles, si servi della sezione delle Pecotteridi sfenotteroidi per fondare il genere Coniopteris, riunendovi alcune forme intermedie fra le Pecopteris e le Sphenopteris, e vi annoverò anche la presente dandole il nome di Coniopteris athyrioides. Ma tutte queste separazioni generiche fondate principalmente sulla forma delle pinuule andranno soggette a rilevanti modificazioni quando ci sarà dato rinvenire esemplari muniti della fruttificazione. Perciò reputo miglior consiglio, a fine di non moltiplicar soverchiamente i nomi, lo attenermi alla nomenclatura proposta dal Presl, e ritenuta da Sternberg, Morris ed Unger.

\section{Splhenopteris Münsteriana. Göpp.}

Sph. fronde bipinnata; pinnis ovatis lanceolatis sessilibus patulis; pinnulis alternis sessilibus oblongis pinnatifidis, laciniis brevibus obtusissimis uninerviis; rachide tenui subalata.

Sphenopteris Münsteriana. Görp. Beitr. zur Flora d. ob. oder weissen Jura, in Uebers. d. Arbeit. d. Schles. Gesellsch. im Jahr. 1845. pag. 149. tab. II. fig. 8. 9. - Ung. Gen. et Spec. Plent. foss. pag. 124. - Zıøo, Enum. Filic. foss. form. Oolith. pag. 20.

In calcareo scissili lithographico formationis oolithicae ad Solenhofen Bavariae.

Questa specie è brevemente descritta e figurata in una Nota che fa seguito alla Mlemoria del chiarissimo Professore Göppert sulla Flora fossile dei terreni Gimrassici medii della Slesia superiore e che trovasi inserita nel Rendiconto dei lavori della Società Slesiana di patria coltura per l'anno 1845. 
Il saggio figurato ci presenta tre pinne quasi parallele uscenti da una rachide piuttosto sottile e che mostrano di avere appartenuto ad una fronda bipinnata. Sono desse sessili, ovato lanceolate e piuttosto patenti, lunghe dieci millimetri e larghe appena cinque, e composte di pinnule sessili, alterne, un po'bislunghe e pinnatifide, le cui lacinie brevissime e molto ottuse sono percorse nel mezzo da un solo nervetto. La rachide sottilissima apparisce lateralmente alata, come si scorge nell'ingrandimento delineato alla fig. 9. della Tav. II. che accompagna la citata memoria del Göppert.

Fu rinvenuta dal celebre naturalista il Conte di Münster, alla cui memoria dedicolla il Prof. Göppert, ed è il primo saggio di una Felce che siasi trovato nella calcarea lilografica di Solenhofen, tanto abbondante di Fucoidi e di Conifere.

\section{Splemoptenis angunta Pomel.}

Sph. fronde tripinnata, rachidibus laevibus, erectis rigidiusculis, subtus profunde canaliculatis; pinnulis obliquissimis linearibus remotiusculis, paucilobis; lobis linearibus angustis, rachidi adpressis, apice aculis, terminali longiore lineari-lanceolato acutissimo; nervis simplicibus.

Sphenopteris angusta. Pomel, Matériaux pour servir à la Flore foss. des ter. jurass. de la France, in Amtl. Bericht über die fünfundzwanzigste Versamml. der Gesellsch. Deulsch. Naturf. und Aerzle in Aachen im Septemb. 1847. pag. 337. n. 1. - Z1Gno, Enum. Filic. foss. form. Oolith. pag. 20.

In formatione oolithica media coralliana ad S.' Michel in Gallia.

Secondo il Pomel l'esemplare porge l'aspetto di una fronda che doveva essere per to meno tripinnata. Le rachidi sottili erette di consistenza piuttosto rigida mostrano sul loro rovescio una profonda scanalatura longitudinale. Le pinnule di cui sono fornite hanno forma lineare ed una disposizione molto obbliqua. Sono poco tra loro avvicinate e si suddividono in lobi poco numerosi lineari, appena divergenti dalla rachide in guisa da raffigurare dei denticelli acuti. Il lobo terminale delle pinnule ha maggiore dimensione degli altri, ed è di forma lineare lanceolata coll' estremità acutissima. Malgrado la piccolezza di questi lobi si possono discernere i nervetti, che sono semplici in cadauna partizione dei lobi stessi.

Questa specie fu rinvenuta nella calcarea del terreno oolitico coralliano a S.' Michel in Francia, e fu brevemente descritta dal Dott. Pomel nella sua Memoria intitolata: 


\section{$-83$}

Matériaux pour servir à la Flore fossile des terrains jurassiques de la France, presentata alla adunanza dei Naturalisti e Medici Alemanni che ebbe luogo in Aquisgrana nel Settembre 1847 e di cui si pubblicarono gli Atti soltanto nel 1849.

\section{Sphenopteris Iobophylla Pomel.}

Sph. fronde tripinnata; pinnis divaricatis plus minusve contiguis; pinnulis pinnarum inferiorum, 7-8 lobatis, lobis ovato-lanceolatis, apice obtusis; mediarum, triphyllis, lobis rotundatis; pinnarum terminalium, simplicibus; rachidibus crassiusculis; nervis divaricatis, bifureatis; ramulo medio elongato.

Sphenopteris lobophylla. Posed, Matériaux pour servir a la Flore des ter. jurass, de la France, in opere citato, pag. 337, n. 2. - Zigro, Enum. Filic. foss. form. Oolith. pag. 21.

Eodem in loco cum praecedenti.

Il Dott. Pomel indica questa specie come spettante al gruppo delle Sfenotteridi a foglioline trilobate. Varia però molto in essa il numero dei lobi in cui si frastagliano le pinnule. In fatti nelle divisioni inferiori della fronda le pinnule di forma ovale oblunga si dividono in sette od otto lobi decrescenti dalla base all' apice della pinnula. Nelle parti mediane sia della fronda che delle grandi pinne primarie non hanno piu di tre lobi che gradatamente si fanno meno distinti e passano superiormente a formare delle pinnule semplici, che divenendo più piccole e più ravvicinate, costituiscono nuovamente nelle pinne superiori, delle pinnule simili a quelle della parte inferiore della fronda, ma appartenenti ad un ordine di suddivisione meno elevato. Le pinne, sebbene più o men contigue alla base, sono però molto divergenti l' una dall'altra, come pure le pinnule i cui lobi sono ovali-lanceolati ad apice ottuso e, nelle trilobate, rotondato. Le rachidi sono robuste ed erette, i nervetti divaricati e biforcati, col ramoscello mediano prolungato a guisa di nervo primario.

Varii saggi di questa specie si trovarono nella calcarea del piano coralliano a S.' Michel in Francia, ma al dire del Dott. Pomel le variazioni nelle frastagliature delle pinnule ne rende assai malagevole la esatta determinazione. 


\section{Spluenopteris Michelinii Pomel.}

Sph. fronde...., pinnis...., pinnulis remoliusculis lanceolatis plus minusve profunde lobatis; lobis ovato-lanceolatis decrescentibus, inferioribus obtusiusculis, superioribus obtusis, terminali latiore simplici vel lobulato; nervis 1-2-5 furcatis; nervulis simplicibus vel bifurcatis.

Sphenopteris Michelinii. Poné, Matériaux pour servir à la Flore des ler. jurass. de la France, in Opere cilato, pag. 338, n. 3. - Zigso, Enum. Filic. foss. form. Oolith. pag. 21.

In formatione coralliana seriei oolithicae mediae prope Chateauroux in Gallia.

Nella descrizione il Dott. Pomel omette di favellare della forma generale della fronda e delle pinne, ma solo osserva come questa specie potrebbe prender posto in un gruppo di cui fosse tipo la Sphenopteris macrophylla del Brongniart. Da ciò si può arguire che gli esemplari da lui esaminati gli abbiano porto indizj sufficienti per ritener che la fronda ne fosse bipinnata.

Le pinnule, al dire del Pomel, sono piuttosto fra loro discoste, di forma lanceolata e partite in lobi più o meno profondamente separati da vani distinti che non giungono però fino alla rachide. Questi lobi hanno forma più o meno ovato-lanceolata e decrescono rapidamente di grandezza. L'apice alquanto outuso nei lobi basilari lo è assai più negli altri, fra i quali il lobo terminale si mostra più largo, talvolta semplice, e talvolta suddiviso in lobuli. Dai nervi escono dei nervelti una, due, e persino tre volte forcuti, secondo la grandezza dei lobi che percorrono, ed i ramoscelli in cui si partono questi nervetti, ora son semplici, ora biforcati anch'essi.

I saggi di questa specie provengono dai calcari della formazione oolitica coralliana delle vicinanze di Chateauroux in Francia.

\section{Sphenopteris pennatula Pomel.}

Sph. fronde tripinnata; rachide valida; pinnis elongatis patentibus approximatis; pinnulis contiguis sublinearibus, lanceolatis, basi angustatis; apice acutis subaequalibus.

Sphenopteris pennatula. Poner, Matériaux pour servir à la Flore des ler. jur. de la France, in opere citato, pag. 338, n. 4. - Zigio, Enum. Filic. foss. form. Oolith. pag. 21. 


\section{In formatione oolithica coralliana apud S.' Michel Galliae.}

Questa specie è rimarchevole, al dire del Dott. Pomel, per la lunghezza delle sue pinne di terz' ordine che sono assai divergenti dalla rachide, ma ravicinate fra di loro. Esse sono fornite di pinnule contigue lanceolate e quasi lineari ristrette alla base, appuntate all'apice e quasi tutte di eguale lunghezza fino all'estremita della pinna. Le rachidi sono piuttosto robuste specialmente le primarie. Della nervatura il Sig. Pomel non fa alcun cenno, per cui è a ritenersi che non abbia trovati esemplari in cui potesse distinguersi.

Anche la presente specie fu rinvenuta nelle roccie calcari spettanti al piano coralliano dell' oolite media presso S. ${ }^{t}$ Michel nella Francia.

In un' Opera che ha per iscopo la Flora generale dell' oolite, non si potevano omettere le piante di questa formazione annoverate dal Pomel nella Memoria sulla Flora giurassica della Francia, ch' egli presentò al Congresso dei Naturalisti e Medici Alemanni, ch'ebbe luogo in Aquisgrana nel Settembre del 1847.

E tanto più vi dovevano essere contemplate in quanto che non se ne trova fatta menzione in alcuna delle Opere paleofitologiche comparse di poi.

A dir vero, le descrizioni del Pomel lasciano molto a desiderare, e sebbene io abbia posto ogni studio per renderle un poco più precise, e tali che giungano a dare un' idea dei caratteri distintivi delle specie, tuttavia è ben difficile che io possa esservi riuscito, non avendo avuto sott'occhio nè figure, nè esemplari su cui istituire gli opportuni confronti.

Riuscirono vane tutte le ricercle da me fatte per ottenere Inmi maggiori sulle piante indicate dal Pomel, come proprie dell'oolite, ed è veramente a deplorarsi che avendo egli di poi rivolto i suoi studj ad altri rami della Paleontologia, abbia tralasciato di dare alla luce una più particolareggiata illustrazione di materiali così importanti.

10. Sphenopteris modesta Bean, MS.

Sph. fronde bipinnata, late lanceolata, apice acuminata; pinnis sessilibus, suboppositis, patentibus, lineari-lanceolatis elongatis; pinnulis alternis, obliquis, ovatis, obtusis, tota base rachidi adnatis, obsolete lobatis, lobis confluentibus crenulato-dentatis; nervis bi-trifureatis, nervulis divaricatis. 
Sphenopteris modesta. Beax, MS. Lecrexbr, On the Sandst, and Shales of the Ool. of Scarb., with descript. of some new spec. of Foss. plants, in Quart Journ. Geol. Soc. Lond. Vol. XX, pag. 79, Tab. X, fig. 3, a, b. - Zigno, Enum. Filic. foss. form. Oolith. pag. 24.

In stratis seriei oolithicae inferioris ad Cloughton et ad Gristhorpe prope Scarbo. rough in comitatu York Angliae.

Una fronda bipinnata ampiamente lanceolata, che nella sua maggiore larghezza misura poco men di sette centimetri e che progredendo verso l'apice si fa lentamente acuminata, distingue questa bella specie. Dalla rachide, che nella parte inferiore del saggio ha un diametro di appena due millimetri e corre ritta assottigliandosi verso l'estremità, escono ad angolo retto le pinne, sessili ed assai patenti, di cui le inferiori sono appena alterne, e le superiori opposte. Tutte poi hanno forma lineare-lanceolata molto allungata e lievemente attenuata verso l'apice, che è ottuso. Le maggiori raggiungono una lunghezza di tre centimetri e mezzo ed hanno una larghezza di otto millimetri al più. Le pinnule di cui sono fittamente guernite, sebbene nella figura appariscano opposte, sono dal Sig. Leckenby indicate come alterne. Inserite obbliquamente con tutta la base sulla rachide, di forma ovata-ottusa un po' rotondata, con appena un leggero indizio di divisione in lobi quasi totalmente confluenti, e col margine lievemente crenulato-dentato, misurano quattro millimetri di lunghezza sopra due o poco più di larghezza. Il nervo sottile che le percorre, e che al punto d'inserzione è collocato un po' fuori del centro della pinnula, si biforca fino a tre volte, risolvendosi in nervetti o ramoscelli assai divaricati.

Questa specie s'incontra frequentemente nelle arenarie della serie oolitica inferiore a Cloughton ed a Gristhorpe presso Scarborough nella Contea di York. Essa fu descritta e figurata per la prima volta dal Sig. Leckenby in una Memoria letta alla Società Geologica di Londra il 17 Giugno del 1863, ed inserita nel fascicolo di Febbrajo 1864 del Giornale trimestrale di quella Società. E fra le piante che essendo comuni agli strati fitolitiferi tanto di Gristhorpe che di Cloughton servirono al Sig. Leckenby per comprovare l'appartenenza di questi due depositi allo stesso orizzonte.

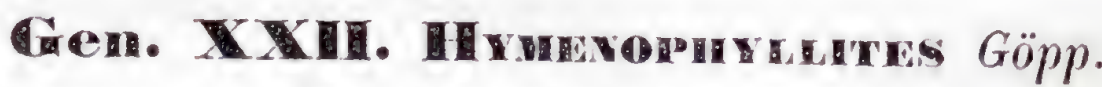

Frons tenera membranacea, bi-vel tripinnata, nunc irregulariter incisorlobata, nunc pinnatifida, pinnis vel laciniis in rachide 
substricta filiformi decurrentibus. Nervi pinnati excurrentes, in singula lacinia solitarii rarius dichotomi. Sori subrotundi, loborum laciniarum apicibus insidentes.

Görp. System. Filic. foss. pag. 251; Gatt. foss. pflanz. pas. 53. - Broxg. Tabl. Gen. Végét. foss. pag. 20. - UxGer. Synops. pag. 69; Gen. et Spec. Plant. foss, pag. 128.

\section{Itymenophyllides maerophyllus Göpp.}

H. fronde bipinnata; pinnis alternis remotis, petiolatis, patentibus, irregulariter pinnatifidis; laciniis distantibus, late linearibus obtusis uninerviis; rachide alata, nervis pinnatis simplicissimis.

Hymenophyllites macrophyllus. Görr. System. Filic. foss. pag. 262. - Uraer. Synops. pag. 71 ; Gen. et Spec. Plant. foss. pag. 131. - linong. Tabl. Gen. Végét. foss. pag, 20 et 105. - Zigno. Enum. Filic. foss. form. Oolith. pag. 21.

Sphenopteris? macrophylla. Brong. Prodrom. pag. 51; Hist. Végét. foss. I. pag. 212. Tab. 58, fig. 3. - Stenne. Vers. Fil. Vorw. II. pag. 63.

Rhodea macrophylla. Prese, in Stenam. Vers. Fl. Vorw. II. pag. 111. - Monnas. Cal. Brit. foss. pag. 19.

In schisto calcarifero formationis oolithicae inferioris ad Stonesfield Angliae, nec non in calcareo jurassico ad Morestel prope Lugdunum Galliae.

I caratteri cbe si possono desumere dal frammento di cui ci porge la figura l'illustre Brongniart, inducono a ritenere che questa specie avesse un' ampia fronda bipinnata, colle pinne alterne, distanti l'una dall'altra venticinque millimetri, verso la base foggiate a picciuolo, ed irregolarmente pinnatifide, colle lacinie discoste fra loro, lineari, oltuse all' apice e percorse da un solo nervo mediano. La sola lacinia che si scorge completa sul saggio, misura due millimetri e mezzo in larghezza sopra venti di lunghezza.

Secondo il Brongniart l'esemplare rinvenuto nella calcaria oolitica coralliana di Morestel presso Lione è di gran lunga più conservato di quello esistente nel Museo d'0xford e che proviene da Stonesfield; ed è veramente a deplorarsi che non se ne abbia alcuna descrizione o figura che giovar possa a meglio precisare $\mathrm{i}$ caratteri generali della specie. 
Non è poi vero che il Presl, collocandola nel genere Rhodea, la abbia posta fra le Alghe, poichè il genere Rhodea fu creato da Presl per abbracciarvi alcune felci che non gli sembravano fornite di caratteri sufficienti per essere annoverate fra le Imenofilliti di Göppert, nè manco fra le Trichomaniti dello stesso autore, nè infine per essere riunite al genere Sphenopteris del Brongniart.

Il genere Rhodea, cui Presl aveva data troppa estensione, fu dall' Unger circoscritto a due sole specie, l'una del terreno carbonifero della Boemia, l'altra, del Keuper delle vicinanze di Bamberga.

Questa specie trovata tanto a Stonesfield, che a Morestel, è l'unica fra le Felci che si palesi promiscua ai depositi oolitici d'Inghilterra, ed a quelli della Francia.

\section{Mymenoplayllites crenulatus Göpp.}

H. fronde pinnata (bi-vel tripinnata?), pinnis alternis sessilibus oblongis obtusis, superioribus acuminatis, profunde pinnatifidis, laciniis obovatocuncatis, apice truncatis, bi-trilobatis, crenulato-dentatis; rachide alata, nervis pinnatis, nervulis secundariis furcatis.

Hymenophyllites crenulatus. Görp. Syst. Filic. foss. pag. 255.-- Uxgrn. Synops. pag. 69 : Gen. et Spec. Plant. foss. pag. 129. - Zigno. Enum. Filic. foss. form. Oolith. pag. 21.

Sphenopteris crenulata. Broxs. Prodrom. pag. 50 ; Hist. Végét. foss. I. pag. 187, Tab. 56 , fig. 3; Tabl. Gen. Végét. foss. pag. 105. - Sterar. Vers. Fl. Vorw. II. pag. 60. - Morms. Cat. Bril. foss. pag. 22.

Dicksonia Brongniarlii. Eтtrssgr. Farnkr. der Jetzwell etc. pag. 217.

In arenaria inferiori formationis oolithicae ad Cloughton in Comitatu York Angliae.

Si può ragionevolmente dedurre, dalla forma e disposizione dei due frammenti collocati sul saggio figurato nell'opera del Brongniart, che questa specie avesse la fronda piuttosto grande e tripinnata. Le pinne sono sessili, alterne, distanti fra loro circa otto millimetri e generalmente tronche all' estremità. Le superiori però si fanno acuminate pella graduata decrescenza delle pinnule verso l'apice. La lunghezza delle pinne sta fra i quindici ed i venti millimetri, e la larghezza tra gli otto ed i dieci. Ogni pinna è profondamente pinnatifida colle lacinie obovato-cuneate, che all' apice si allargano e finiscono coll' estremità bruscamente tronca, frastagliandosi in due o tre lobi brevissimi, crenulato-dentati. La rachide è brevemente alata, largamente to sono le rachidi secon- 


\section{- $89-$}

darie pella continuità della base delle lacinie. I nervi sono disposti in forma pinnata ed i nervetti secondarii si biforcano in corrispondenza ai denticelli della estremità.

Osserva il Brongniart come questa specie debba considerarsi fra le meno frequenti nei depositi oolitici d'Inghilterra, poichè nel IIuseo della Società filosofica di York, ebbe solo a vederne dei piccoli frammenti, fra i quali prescelse il Saggio di cui ci porse la figura, e clie è abbastanza conservato per potervi riconoscere la forma e suddivisione delle pinne e delle pinnule e la distribuzione dei nervi, non però sufficiente a dare un'idea complessiva della forma generale della fronda.

Il Sig. Leckenby annovera questa specie fra quelle che si trovano esclusivamente nel deposito fitolitifero di Cloughton.

Il Professore Costantino di Ettingshausen nella recente sua Opera intitolata: Die Farnkreuter der. Jetzwelt zur untersuchung und bestimmung der in den formationen der Erdrinde eingeschlossenen überreste von vorweltlichen Arten dieser ordnung: descrive questa specie dandole il nome di Dicksonia Brongniarti, e facendo rilevare le analogie che presenta confrontandola con alcune Dicksonie della Flora attuale (Dicksonia apiifolia Sw.; D. expansa Kaduf.; D. rubiginosa Kaulf.). Non v'ha dubbio che nell' aspetto generale, e nella distribuzione dei nervi, essa palesi realmente una qualche analogia colle Dicksonie viventi. Però, mancando il saggio di ogni traccia di fruttificazione, non credo si possa per ora ammettere, in modo cosi riciso, il collocamento di questa specie nel genere Dicksonia.

\section{Tymenophyllites Williamsonis Göpp.}

H. fronde bipinnata; pinnis distantibus, sessilibus, subalternis saepius oppositis, patentibus; pinnulis suboppositis flabellatim digitato-quadrifidis, quandoque profunde et inaequaliter bifidis, basi cuneato-attenuatis, laciniis linearibus lobatis, dentatis, integrisve apice truncatis; rachidibus anguste. alatis, nervis tenuissimis aequalibus parallelis.

Hymenophyllites Williamsonis. Görp. Syst. Filic. foss. pag. 259. - Ung. Synops. pag. 70 ; Gen. et Spec. Plant foss pag. 130. - Zigno. Enum. Filic. foss. form. Oolith pag. 21.

Sphenopteris Williamsonis. Brosg. Prodrom. pag. 50; Hist. Végét. foss. I. pag. 177, Tab. 49, fig. 6, 7, 8; Tabl. Gen. Végét. foss. pag. 105. - Lindu. Hutt. Foss. Flor. Gr. Bril. II. Tab. 131, pag. 439. - Sтеккв. Vers. Fl. Vorw. II. pag. 58. 


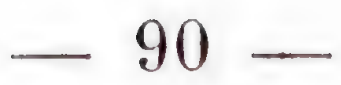

Sphenopteris digitata. Punctrs. Geol. Yorksh. pag. 152, Tab. 8, fig. 6, 7.

Rhodea Williamsonis. Prest in Stensв. Vers. Fl. Vorw. II. pag. 110, - Monris. Cat. Brit. foss. pag. 19.

In formatione oolithica ad Gristhorpe Bay prope Scarborough Angliae.

Nella descrizione di questa specie io mi attengo alle figure pubblicate da Phillips e da Lindley ed Hutton, come quelle che meglio rappresentano i caratteri distintivi della specie, perchè eseguite sopra esemplari più conservati di quelli che servirono per l'Opera del Brongniart. In esse la fronda ci si palesa bipinnata, colle pinne inserite a qualche distanza l'una dall'altra, rade volte alterne, più spesso opposte, sessili, assai patenti e lunghe dai tre ai quattro centimetri. Le pinnule, quasi sempre opposte, e verso la base cuneato-attenuate, si suddividono talvolta irregolarmente in tre o quattro lacinie lineari di forma digitato-flabellata; più di sovente però sono profondamente bifide. Verso le estremità sono, o dentate, o partite in lobi lineari in cui l'apice si mostra sempre o ottuso o tronco. Le rachidi appariscono brevemente alate, ed i nervi sono esilissimi, eguali, e paralleli.

Questa specie si trova piuttosto di rado nelle arenarie della formazione oolitica presso Scarborough nella Contea di York ed è posta dal Sig. Leckenby fra le specie proprie del deposito di Gristhorpe Bay.

La maggior parte degli autori che descrissero questa specie, il fecero sulle figure date dal Brongniart, e citando quelle della Fossil Flora of Great Britain dei Signori Lindley e Hutton, commettono l'errore d'indicare la Tav. 31 del Volume II, in luogo della Tav. 131 dello stesso Volume ove ne sono figurati due esemplari, ed è pure delineato l'ingrandimento microscopico della porzione terminale di una pinnula in cui si ravvisano con tutta chiarezza i nervetti finissimi e paralleli che la percorrono in senso longitudinale.

\section{Iymenophyllites Dillipsii Göpp.}

H. fronde tenui bipinnata; pinnis sessilibus, alternis, distantibus, patentissimis, lineari-lanceolatis elongatis; pinnulis alternis, approximatis, adnatosessilibus in rachidem alatam decurrentibus, ovato-oblongis, pinnatifidis; laciniis sterilibus, obliquis, apice trilobis, inferiori interna, majori, lobis tri- 
dentatis; laciniis fertilibus brevismis, simplicibus, raro bifidis, apicibus fructiferis dilatatis, indusio reniformi, instructis; nervis pinnatis simplicibus.

Hymenophyllites Phillipsii. Görp. Syst. Filic. foss. pag, 256. - Urg. Synops. pag. 70 ; Gen. et Spec. Plant. foss. pag. 129. - Zigno. Enum. Filic. foss. form. Oolith. pag. 21.

Sphenopteris hymenophylloides. Brong. Prodr. pag. 51; Hist. Végét. foss. I. pag. 189, Tab. 56 , fig. 4; Tabl. Gen. Végét. foss. pag. 105. - Sterns. Vełs, Fl. Vorw. II. pag. 60. - Monnus. Cat. Brit. foss. pag. 22.

Sphenopteris stipata. Pullurs. Geol. Yorksh. pag. 153, Tab. 10, fig. 8.

Sphenopteris nephrocarpa. Bensury. Foss. Pl. from the Jur. strata of the Yorksh. Coast; in Quart. Journ. Geol. Soc. Lond. Vol. ViI. pag. 179, Tab. 12, fig. 1.

Hymenophyllites nephrocarpos. Zigso. Enum. Filic. foss, form. Oolith. pag. 22.

Dicksonia hymenophylloides. Eттікgsm. Farnkr. etc. pag. 217.

In arenaria formationis oolithicae ad Egton Moors, Saltwick, Gristhorpe et ad IIaiburn Wyke Angliae.

Questa specie che non deve confondersi colla Sphenopteris Phillipsii di Mante!l del terreno Wealdiano di Heathfield e Wilts (Vedi Mantell. Geol. of the S. E. of England pag. 239), ci presenta una bella fronda bipinnata colle pinne sessili, alterne, distanti, assai patenti, di forma lineare lanceolata, lunghe dai cinque ai sei centimetri e larghe alla base ben quindici millimetri. Le pinnule sono pure alterne, ma molto avvicinate tra loro, lunghe appena nove millimetri e larghe da tre a quattro, di forma ovata-bislunga e pinnatifide, riunite per la base e decorrenti in guisa da formar ala alla rachide. Le lacinie in cui si suddividono sono oblique e verso l'apice partite in tre lobi eguali di cui il medio è più sporgente, ma la inferiore interna di cadauna pinnula è assai più grande dell'altre ed i lobi più ampi in cui è frastagliata hanno le estremità tridentate, coi denti ottusi. Le lacinie poi delle pinnule, fornite della fruttificazione, sono allo invece semplicissime ed assai abbreviate, talvolta bipartite all'apice e colle estremità dilatate e fornite di un ingrossamento marginale rotondato e reniforme che ha tutta l'apparenza di un indusio contenente la fruttificazione.

La rachide è alata ed i nervetti pinnati, coi ramoscelli semplici percorrenti ogni singolo lobo delle lacinie.

Il Professore Phillips indica questa specie come proveniente dalle arenarie inferiori della serie oolitica di Egton Moors, di Saltwick e di Haiburn Wyke. Brongniart ne 
cita la frequenza negli schisti carboniferi della stessa serie presso Whitby. Leckenby la annovera nel suo elenco delle piante fossili dei depositi oolitici di Gristhorpe e di Cloughton.

Nel mio Catalogo delle Felci fossili dell'oolite, io tenni la Hymenophyllites Phillipsii Göpr. separata dalla Sphenopteris nephrocarpa di Bunbury; ma la opinione, posta innanzi dal Sig. Leckenby, che quest'ultima altro non fosse che lo stato fertile della prima, m'indusse a prendere nuovamente in accurato esame i caratteri dell' una e dell'altra per investigarne sotto questo punto di vista le affinità. E per vero, la forma e suddivisione delle pinnule, il modo con cui s'uniscono decorrendo nel margine alato della rachide, la distribuzione dei singoli nervetti nei lobi delle lacinie, l'ingrossamento reniforme dell'apice di quest'ultime, quale si osserva nella Sphenopteris nephrocarpa Busi., palesano esistere, fra queste due forme, quelle stesse analogie che si riscontrano paragonando fra di loro le pinnule sterili, e quelle munite della fruttificazione, nelle Dicksonie della Flora attuale.

L'illustre Brongniart aveva già rimarcato nella sua Sphenopteris hymenophylloides, una grande rassomiglianza con varie specie del genere Dicksonia, e dopo di lui il chiarissimo Cavaliere Bunbury, illustrando la sua Sphenopteris nephrocarpa faceva rilevare le stesse analogie ed a maggior prova di ciò porgeva a canto alla figura di quella specie, il disegno di una pinna fruttifera della Dicksonia coniifolia Iоок, ora vivente nella Nuova Granata, che a dir vero molto si avvicina all' esemplare della sua specie fossile.

Dall' esame di tutti questi caratteri, e di quanto scrissero Brongniart e Bunbury sopra queste analogie, mi sembra che il parere esternato dal Sig. Leckenby acquisti tale grado di probabilità, da giustificare per ora la riunione di queste due specie, ritenendo la Sphenopteris nephrocarpa di Bunbury per lo stato fertile della Sphenopteris stipata di Phillips o Hymenophyllites Phillipsii di Göppert.

\section{Hymenophylites Murrayana Zign.}

H. fronde bi-tripinnata, rachidibus laevibus flexuosis; pinnis sterilibus, alternis, sessilibus, ovato-lanceolatis elongatis, superioribus tantum pinnatis, inferioribus bipinnatis; pinnulis basi contractis ovato-triangularibus obtusis, crenatis vel pinnatifidis, lobis obliquis ovato-lanceolatis oblusis, nervulis bipinnatis parenchymate immersis; pinnis fertilibus ad meras rachides reductis, pinnulis abrupte pinnatis, laciniis brevibus oppositis, remotiusculis, linearibus angustissimis, apice in disco subhemisphaerico dilatatis. 


\section{$-93-$}

Pecopteris Murrayana. Brong. Hist. Végét. foss. I. pag. 358, Tab. 126, fig. 1-5. - Urgen. Synops. pag. 99 ; Gen. et Spec. Plant. foss. pag. 179.

Polystichites Murrayana. Prest in Stennis. Vers. Flor. Vorw. II. pag. 117. - Monnis. Cal. Brit. foss. pag. 19.

Coniopteris Murrayana. Brong. Tabl. Gen. Végét. foss, pag. 26 et 105.

Sphenopteris Murrayana. Zıgxo. Enum. Filic. foss. form. Oolith. pag. 20.

Tympanophora racemosit. Linds. Hutw, Foss. Flor. Gr. Brit. Vol. III, pag. 170, Tab. 170 B.

- Lecrensy. On the sandst. and Shales of the Ool. of Scarb. with descript. new spec. foss. plants, in Quart. Journ. Geol. Soc. Lond. Vol. XX, pag. 79, Tab. XI, fig. 2.

In arenaria inferiori formationis oolithicae ad Gristorpe et ad Cloughton mrope Scarborough Angliae.

Da quanto si può desumere esaminando i varii saggi di questa specie, figurati nell'opera del Brongniart, e quelli esistenti nel mio Gabinetto, dobbiamo ritenere che la fronda superiormente bipinnata, si frastagliasse in forma tripinnata verso la base.

La rachide primaria si mostra liscia ed ha un diametro di appena due millimetri. Le rachidi secondarie sono assai sottili e flessuose. Le pinne sono alterne, sessili, patenti, ovato-lanceolate, e molto allungate, essendovene taluna che oltrepassa gli otto cenlimetri sopra una larghezza di venticinque millimetri. Le superiori, che van decrescendo verso l'apice della fronda, sono semplicemente pinnate, bipinnate le inferiori. Le pinnule, ristrette alla base, sono ovato-triangolari, ottuse, crenate, o pinnatifide, coi lobi ovato-lanceolati oltusi, ed un poco obliqui. Il nervo mediano che le percorre, si suddivide in nervelti secondarii pinnati, poco visibili perchè immersi nel parenchima.

Appoggiato al parere autorevole di varii distinti paleofitologi, io riunisco a questa specie quella forma problematica che i Signori Lindley e Ilutton figurarono e descrissero sotto il nome di Tympanophora racemosa, e che il Pomel collocò fra le Alghe, credendo ravvisarvi qualche somiglianza col Fucoides turbinatus, e col Fucoides discophorus del Brongniart.

Fino dal 1850, quest'ultimo illustre naturalista, nell'opera intitolata Tableau des genres des Végétaux fossiles, faceva rilevare l'affinita esistente fra la Tympanophora racemosa L. II., e la Thyrsopteris elegans Kunze dell' Isola di S. Juan Fernandez, in in cui la parte fertile della fronda è mancante di parenchima, e ridotta alle sole rachidi, le cui suddivisioni portano sulle loro estremità degli indusii in forma di coppa.

Sir Charles Bunbury, nella sua Memoria sopra alcune piante fossili degli strati giuresi della Contea di York (Quart. Journ. Geol. Soc. Lond. Vol. VII. pag. 181), dopo 


\section{$-94-$}

avere accennato, come i magnifici esemplari della Tympanophora racemosa L. II., esistenti nella collezione del Sig. Bean, palesino chiaramente una decisa analogia collo stato fertile delle Dicksonie e delle Imenofillee, riferisce essere opinione dello stesso Sig. Bean, che questa forma altro non sia che lo stato fertile della Pecopteris (Hymenophyllites) Murrayana Brosa.

È bensi vero che il Brongniart, nella Histoire des Végétaux fossiles, fra le varie figure di questa specie contenute nella Tav. 126 , ci porse la fig. 5 . A, in cui si osservano sopra una pinnula tre protuberanze circolari ombilicate nel centro, che rammentano gli indusii orbiculari peltati di alcune Aspidiacee; ma nel testo l'autore non entra in alcun particolare su questo proposito, e dopo che si trovarono a Scorborough degli esemplari di questa specie colle pinne sterili, unite alle fertili sullo stesso individuo, uno dei quali fu pubblicato dal Sig. Leckenby, nella citata Memoria sull'Oolite di Scarborough, colla fig. 2. della Tav. 11. (Quart. Journ. Soc. Geol. Lond. Vol. XX. pag. 79), è gioco forza ritenere, che quell' apparenza di sori, di cui ci diede la figura il Brongniart, sia invece dovuta alla presenza di qualche miceto epifillo.

Il saggio figurato dal Sig. Leckenby è sommamente istruttivo, e toglie ogni dubbio, poichè ci presenta porzione di una fronda fornita di cinque pinne sterili, evidentemente spettanti alla specie di cui trattiamo, e di tre pinne fertili, che per la forma delle pinnule corrispondono a puntino con quelle della Tympanophora racemosa L. H.

Sono quindi le pinne fertili della Hymenophyllites Murrayana emaciate e ridotte alle sole rachidi. La forma ne è pinnata e tronca all' estremità. Le pinnule in cui si dividono sono menomate e ristrette in guisa da potersi piuttosto considerare quali lacinie lineari angustissime. Son desse collocate rimpetto l' una dell'altra con qualche distanza fra cadaun pajo. L'estremità poi di ogni lacinia si espande e si dilata formando così una specie di disco semisferico che presenta qualche analogia colla fruttificazione della Thyrsopteris e di alcune Dicksoniacee.

Dopo il fin qui detto, non credo si possa muover dubbio sull'appartenenza di queste due forme alla medesima specie, ed egualmente sono di parere, che esaminando il complesso dei loro caratteri, si troverà giustificato il collocamento della specie nel genere Hymenophyllites.

Anche questa specie è annoverata dal Sig. Leckenby fra quelle che sono commi tanto ai depositi di Gristhorpe, che a quelli di Cloughton presso Scarborough.

Prima che le osservazioni dei Signori Bean e Leckenby, mettessero in chiara luce la vera natura della Tympanophora racemosa, e l'intima sua relazione colla Pecopteris (Hymenophyllites) Murrayana, il Sig. Pomel, nella più volte citata sua Memoria sulle 
piante giurassiche della Francia, presentata al Congresso dei Naturalisti Alemanni ch' ebbe luogo in Aquisgrana nel 1847, poneva, come dissi, il genere Tympanophora fra le Alghe, riunendovi la Sphatereda paraloxa di Lindley e Ilutton, il Fucoides turbinatus ed il $\boldsymbol{F}$. discophorus del Brongniart, e la Gaulerpites sphaeroides del Münster, come pure due nuove specie del terreno coralliano di S.' Michel e di Seyssel, da lui denominate Tympanophora conferta e T. irregularis, e contraddistinte colle frasi specifiche che si leggono negli Atti di quel Congresso alla pag. 335.

Credo opportuno richiamare su quest'ultime l'attenzione dei Naturalisti che fossero in grado di procurarsene degli esemplari, imperciocchè, dalle brevi descrizioni del Pomel, non si può con sicurezza decidere, se queste due forme appartener debbano alla classe delle Felci, o a quella delle Alghe.

\section{Hymenophyllites Leckenbyi Zigno.}

Tav. IX. fig. 3, 4. 5., Tav. XIV. fig. 1.

H. fronde tripinnata; rachide stricta subflexuosa sulcata; pinnis alternis elongatis patulis; pinnulis sessilibus alternis, superioribus brevibus, flabellatim 2-4 partitis, reliquis elongatis profunde pinnatifidis; laciniis angustis oppositis, plerumque bifidis, segmentis cuneiformibus divaricatis, apice dilatatis; ncrvulis in singulis laciniis, solitariis, vix conspicuis, in loborum apicibus, furcatis, an sorigeris.

In saxo calcareo cinereo seriei oolithicae inferioris ad Valles Zuliani et Salaorno prope Roverè di Velo in Provincia Veronensi.

La fronda di questa specie è largamente Janceolato-allungata, e si divide in forma tripinnata. Dai varii frammenti raccolti si può dedurre che raggiungesse talvolta una lunghezza di oltre trenta centimetri sopra una larghezza di dieci. La rachide primaria ha un diametro di due millimetri negli esemplari più adulti. Essa corre ritta fino all'apice della fronda, percorsa da un solco longitudinale piuttosto profondo, e solo mostrasi apparentemente flessuosa pegli ingrossamenti clie marcano alternamente i punti d'inserzione delle rachidi secondarie. Queste ultime, alquanto flessuose, sono pure segnate da un solco longitudinale.

Le pinne sono sessili, alterne, e patenti uscendo quasi ad angolo retto dalla rachide principale. Negli esemplari che presentano la parte inferiore della fronda sono discoste 


\section{$-96$}

l'una dall'altra circa dieciotto millimetri, ma verso l'estremità si vanno avvicinando in modo che tale distanza si riduce gradatamente a soli tre millimetri. A seconda poi dell'età, varia la loro lunghezza dai due ai cinque centimetri, e la larghezza dai tre ai dieci millimetri. Le pinnule di cui vanno fornite sono pure sessili ed alterne, ma non presentano tutte la stessa forma. Negli individui giovani ed in tutta la parte superiore che termina la fronda, come pure nelle estremità delle pinne, si mostrano assai brevi e profondamente partite in due divisioni, cadauna delle quali è suddivisa in due segmenti cuneiformi, talvolta triangolari, riuniti fra loro per la parte più angusta e disposti a ventaglio. Tutte le altre pinnule sono assai più allungate e pinnatifide colle lacinie opposte angustissime, per lo più bipartite e coi segmenti, pure cuneiformi, divaricati e dilatati all'apice.

I nervetli, che appena si possono scorgere su qualche saggio ben conservato, appariscono singoli in ogni lacinia, biforcandosi verso le suddivisioni dei lobi nelle cui estremità un certo ingrossamento del tessuto sembrerebbe indicare che ivi fosse collocata la fruttificazione.

A prima giunta questa specie presenta una grande rassomiglianza colla figura della Tympanophora racemosa dei Signori Lindley e Hutton. E per vero nei varii saggi che conservo nella mia collezione si osserva un analogo modo di frastagliarsi della fronda ed un'eguale disposizione delle pinne e delle pinnule. Ma ben diversa è la forma delle lacinie le quali nella presente specie sono cuneiformi e, fuorchè nell' età giovane, costantemente bipartite coi segmenti o lineari o cuneiformi o triangolari; nè giammai s'incontra in essi quell'espansione terminale semisferica e tronca all'apice, che ver'so la base si stringe rapidamente a mo' di pedicello da cui vedemmo distinta la Tympanophora racemosa, e che rammentando l'aspetto della fruttificazione di alcune Felci viventi, faceva presagire la sua appartenenza allo stato fertile di una qualche specie fossile.

Queste differenze ed il fatto di non essermi giammai imbattuto nelle Alpi Venete, durante quattordici anni di accurate ricerche, in alcun minimo frammento della fronda sterile della Pecopteris (Hymenophyllites) Murrayana, m’indussero a considerare questa forma come spettante ad altra specie, proponendo per essa il nome di Hymenophyllites Tympanophoraeformis. Ma siccome per le recenti osservazioni riusci palese la spettanza delle due specie comprese nel genere Tympanophora alla forma fruttifera di due Felci appartenenti a generi conosciuti, cosi ne consegue che il genere Tympanophora debba ora eliminarsi dall' elenco delle piante fossili. Non sarebbe quindi esatto il denominare la presente specie in guisa da accennare alla sua affinita con un genere che non può più sussistere; d'altronde il nome di Hymenophyllites Tympanophoraeformis non essendo mai stato pubblicato, ma solo segnato sulle etichelte degli esemplari della mia 
collezione credetti più opportuno, pubblicandone ora la descrizione, di distinguere questa forma con altro nome, cogliendo in pari tempo l'occasione d'intitolarla al chiarissimo Sig. Giovanni Leckenby, noto per varii interessanti lavori paleontologici e stratigrafici sulla formazione oolitica e sulle piante fossili di Scarborough.

I saggi di questa curiosa felce, furono da me trovati nelle calcaree grigie e giallastre della serie oolitica inferiore, nella Valle Zuliani ed in quella di Salaorno, presso Roverè di Velo nella Provincia Veronese. Però non è tanto frequente ed è cosa assai rara trovarne esemplari ben conservati.

Nella tavola IX. fig. 3. 4. 5. sono delineate alcune porzioni delle estremità della fronda, col loro ingrandimento (fig. 3. a, fig. 5. a). Avendone di poi rinvenuto un saggio colla fronda quasi completa, ne do la figura alla tavola XIV. fig. 1., unitamente all'ingrandimento (fig. 1. b) di porzione della rachide primaria e di una pinna, in cui è espresso il modo di frastagliarsi delle pinnule, e la forma delle lacinie, quali si palesano nelle pinne della parte media ed inferiore della fronda.

\section{Itymenophyllites? prisea Zigno.}

H. fronde pinnata vel bipinnata? pinnis linearibus, rachide tenui, substriata, pinnulis alternis ovato-lanceolatis obtusiusculis, basi constrictis, in rachidem subalatam decurrentibus, profunde septem lobatis, lobis apice lanceolatis obtusis, integerrimis, tribus terminalibus, minoribus; nervis basi divisis, medio longiore excurrente, apice furcato; nervulis tenuibus obliquis, ramulis furcalis.

Sphenopteris prisea, Eicnwald. Lethaea Rossica. Vol. II. pag. 14. Lab. IV. fig. 2. a.

In formatione oolithica (oxfordiana?) ad Kamenka prope Izoume in Russia meridionali.

Il frammento di pinna descritto e figurato dall'Eichwald non esclude il dubbio che la fronda potesse anche essere bipinnata. Questa pinna si mostra dritta, lineare, larga circa dodici millimetri e munita di una rachide sottile e leggermente striata. Le pinnule sono alterne, ovato-lanceolate, coll' estremità piuttosto ottusa, lunghe circa sei millimetri, larghe verso la base quattro millimetri, dopo di che stringendosi decorrono formando ala alla rachide. Esse si dividono in sette profondi lobi, dei quali gli inferiori, due per cadaun lato, sono largamente ed oltusamente lanceolati, ed i tre supe- 


\section{$-98$}

riori, di cui il mediano costituisce l'apice della fronda, sono più stretti e più piccoli, ma egualmente ottusi. I nervi al punto d'inserzione sono fra loro disgiunti ed il mediano si prolunga fin verso l'estremità della fronda, mentre i nervetti secondarii che escono obliquamente in forma pinnata da quello si dirigono ver l'apice dei lobi, dividendosi in ramoscelli forcuti alquanto divaricati.

Questo saggio fu trovato in un calcare giurassico, spettante al gruppo Oxfordiano, a Kamenka presso Izoume nella Russia meridionale.

Questa specie, per la forma generale delle pinne e delle pinnule, ha qualche somiglianza colla Hymenophyllites Phillipsii di Göppert o Sphenopteris stipata di Phillips. Però le divisioni dei lobi delle pinnule sono meno profonde, ed i lobi stessi non si suddividono in altri minori come nella specie di Phillips. Tuttavia l'aspetto complessivo la avvicina piuttosto alle Imenofilliti che alle vere Sfenotteridi.

\section{Gen. XXIII. Dichoptenis Pomel.}

Frons simplex, stipitata, dichotoma, lobata, lobis 6-8, linearibus subflabellatis; stipite tereti, superne canaliculato, medio crassiore; nervis tenuibus, parallelis, aequalibus, in typica specie obsoletis.

Poxer, Malériaux pour servir à la Flore des ter. Jurass, de la France in Amtl. Berich der Gesellsch. Deutsch. Natur, und Erste in Aachen. Sept. 1847, pag. 339. Aachen. 1849.

\section{Dicropteris Iaciniata Pomel.}

D. fronde pollicari, lobis 8 , divergentibus, elongatis, apice attenuatis, obtusiusculis, dichotomiarum angulis remolis, aequidistantibus; nervis obliteratis.

Dichropteris laciniata Ponez. Matér. puur servir à la Flore Jurass. de la France, ete in opere citato pag. 339, n. 1; - Z1Gro. Enum. Filic. foss. form. Oolith. pag. 25.

In formatione coralliana (coral rag), seriei oolithicae mediae apud S.' Michel in Gallia. 


\section{$-99-$}

La fronda è lunga un pollice, e si dicotomizza dividendosi in lobi divergenti, alIungati, attenuati verso l'apice, ove terminano alquanto ottusamente. Gli angoli prodotti dalle dicotomie sono remoti ed equidistanti. In quanto ai nervi, il saggio non ne presenta alcuna traccia.

Questa specie fu trovata in una roccia riferita al piano coralliano della serie oolitica media presso S. Michel in Francia.

\section{Dicropteris Mabelliformis Pomel.}

D. fronde minori, lobis 8 , erectis, subflabellatis, apice dilatatis, obtusis, basi subattenuatis, dichotomiarum angulis approximatis, inaequidistantibus, nervis obliteratis.

Dicropteris laciniata. Porrel. loc. citat. pag. 339, n. 2. - Zigso. Enum. Filic. foss. form. Oolith. pag. 25.

Eodem in loco cum praecedente.

La fronda di questa specie è ancor minore di quella della precedente, e si divide in otto lobi eretti disposti a ventaglio, dilatati ed ottusi all'apice e leggermente attenuati alla base. Gli angoli delle dicotomie son collocati ad ineguali distanze e più ravvicinati. Anche in questo esemplare non si possono distinguere i nervi.

Si trova nella stessa roccia e nel medesimo luogo.

\section{Dicropteris longifolia Pomel.}

D. stipite tereti canaliculato, fronde semipedali, lobis 6 , interioribus simplicibus, omnibus elongatis, erectis, basi attenuatis; dichotomiarum angulis approximatis; nervis tenuibus, parallelis, contiguis (an medio validiore?).

Dicropteris longifolia. Ponet, loc. cital. pag. 339, n. 3. - Zigro. Enum. Filic. foss. form. Oolith. pag. 25.

In formatione Goralliana prope Chateauroux in Gallia. 
In questa specie la fronda è più grande e raggiunge una lunghezza di mezzo piede e si divide in soli sei lobi semplici eretti, allungati ed attenuati verso la base. Gli angoli delle dicotomie sono in essa più approssimati, e lo stipite che la sorregge a guisa di picciuolo è rotondo, ma superiormente scanalato. Soltanto nei saggi di questa specie potè il Pomel discernere i nervi, ch'egli indica essere tenui, semplici, eguali, paralleli ed avvicinati fra di loro, e solo accenna con qualche dubbio come il nervo medio potesse essere stato più degli altri robusto.

Questa specie fu rinvenuta nella medesima formazione nelle vicinanze di Chateauroux in Francia.

Il Pomel parlando brevemente delle affinità che presenta questo suo nuovo genere, esprime l'opinione che debba collocarsi nell'ordine delle Sfenotteridee. A dir vero, la forma della fronda e la distribuzione dei nervi mi avevano indotto ad annoverarlo nel mio nuovo Ordine delle Ciclotteridee (Vedi Zigno, Enum. Filic. foss. form. oolith. pag. 25), ma, non avendo potuto procurarmi l'ispezione degli esemplari, stimai miglior consiglio lasciare questo genere nella famiglia in cui fu collocato dal suo fondatore.

\section{Gen. XXIV. Coxoptenis Pomel.}

Frons tripinnata; pinnulis plus minusve adnatis, integris vel profunde pinnatifidis; nervo inferne marginali; nervulis secundis simplicibus, furcatis pinnatisve.

Ponel. Mém. sur le lias de la Moselle et sur quelques gisements de veget. foss. in Bull. Soc. Géol. de France, Ser. II. Vol. 3. pag. 654 ; Matér. pour la Flore Jurass. de la France etc. in opere citato, pagg. 336.

\section{Loxopteris elegans Pòmel.}

L. fronde tri... pinnata; pinnis contiguis late linearibus, pinnulis falcalis, oblongis, approximatis, in rachidem decurrentibus, angulo superiore subauriculatis, apice obtusis, margine inferiore integris, superiore repandocrenatis; rachide stricta gracili; nervo distincto, nervulis obsoletis.

Loxopteris elegans. Posies. Matér. pour la Flore Jurass. de la France etc., in opere citato pag. 336. - Zıåo. Enum. Filic. foss. form. Oolith. pag. 25. 


\section{$-101-$}

Invenit Clarissimus Moreau in formatione Coralliana apud S.' Michel in Gallia.

La fronda si palesa in questa specie tripinnata colle pinne contigue e largamente lineari e colle pinnule falcate, bislunghe, avvicinate e decurrenti sulla rachide. Nell'angolo superiore della base sono pressochè auricolate; all'apice si mostrano oltuse ed hanno il margine superiore repando-crenato, mentre l'inferiore è invece intero. La rachide corre ritta ed è piuttosto sottile. Il nervo primario delle pinnule è abbastanza distinto, ma i nervetti secondarii non sono visibili.

Il saggio descritto dal Pomel fu trovato dal Sig. Moreau nella formazione Coralliana presso S.' Michel in Francia.

Questo genere di cui il Pomel porgeva l'illustrazione fino dal giugno 1846 (Vedi Bull. Soc. Géol. de France. Serie II. Vol. 3. pag. 654) si distingue principalmente per avere le pinnule obblique e quasi dimidiate, col nervo principale corrispondente al margine inferiore e munito soltanto nel lato superiore di nervetti secondarii semplici o forculi. L' orlo superiore delle pinnule è più o meno profondamente diviso in lobi, mentre l'inferiore è invece interissimo.

L'illustre Brongniart nel più volte citato suo Tableau des genres des Végétanx fossiles, pag. 20, opina che il genere Loxopteris debba prender posto nella stessa famiglia in cui sono collocati i generi Sphenopteris, Hymenophyllites, Trichomanites, ed è per tal ragione che io l' ho qui provvisoriamente annoverato fra i generi dell'ordine delle Sfenotteridee, sebbene $\mathrm{i}$ caratteri assegnati dal Pomel a questo genere non mi sembrino giustificare pienamente tale classificazione.

\section{Ordo X. Cyclopterideae.}

Frons, aut stipitata integra vel incisolobata, aut pinnata vel bipinnata. Pinnae liberae, pinnulis basi cordatis vel adnatis aut decurrentibus. Nervi aequales, simplices vel furcati, omnes ab ima basi flabellati, vel e rachide seriatim exorientes ad apicem marginemve pinnularum excurrentes, ramulis subparallelis. Fructificationes punctiformes sparsae, vel fortasse marginales.

Zigno. Enum. Filic, foss, form. Oolith. pag. 22. 


\section{Gen. XXv. Crchopheris Brong.}

Frons, aut stipitata flabellato-semiorbiculata, integra vel inciso-lobata, aut digitata vel pinnata. Nervi creberrimi ab ima basi flabellati, dichotomo-furcati, aequales, ramulis subparallelis. Fructificatio adhuc dubia, fortasse marginalis.

Brong. Prodrom. pag. 51; Hist. Végél. Foss. I. pag. 215 ; Tabl. des Gen. Végét. foss. pag. 17. - Stenns. Vers. Flor. Vorw. II. pag. 65 (pro parte). - Görp. Gatt. foss. P/l. pag. 89. - Ung. Synops. pag. 53; Gen. et Spec. Plant. foss. pag. 94 (pro parte).

Adiantites. Görp. Syst. Filic. foss, pag. 216 (pro parte).

\section{Cyelopteris digitata Brong.}

C. stipite tenui, supra canaliculato; fronde simplici, flabellato-semiorbiculata, multiloba, in stipitem attenuata, lobis parallelis subaequalibus, contiguis, truncatis, apice repandis incisisve; nervis tenuibus, creberrimis, aequalibus, dichotomis.

Cyclopteris digitata. Brorg. Hist. Végét. foss. I. pag. 219, tab. 61 (bis) fig. 2. 3. - Sterrв. Vers. Flor. Vorw. II. pag. 66. - Urg. Synops. pag. 53; Gen. et Spec. Plant. foss. pag. 94. - Zigro. Enum. Filic. foss. form. Oolith. pag. 22.

Adiantites digitata. Göpr. Syst. Filic. foss. pag. 217.

Sphenopteris latifolia Pnilcips. Geol. of the Yorksh. Coast. I. pag. 152, tab. VII. fig. 18.

In stratis arenaceis et argillosis seriei oolithicae inferioris ad Gristhorpe et ad Cloughton prope Scarborough Angliae.

Da uno stipite assai sottile e superiormente scanalato si espande a ventaglio la fronda, semiorbicolare e divisa più o meno profondamente in lobi cuneiformi, paralleli, contigui, tronchi all' estremità ed ivi irregolarmente sinuosi od inciso-dentati. I nervetti che numerosi sorgono dal punto ove la fronda si fonde nello stipite sono eguali e finissimi, si biforcano ripetutamente e scorrono fino al margine della fronda mostrandosi quasi paralleli fra di loro, ma però gradatamente prendendo una disposizione flabellata in armonia colla forma generale della fronda. 


\section{$-103$}

Nell' esemplare figurato dal Brongniart lo stipite ha un diametro di due millimetri appena, e la fronda ha una larghezza di cinque centimetri, sopra tre centimetri e mezzo di lunghezza. Le divisioni dei lobi non si profondano che fino alla metà della fronda o poco più, ed i lobi stessi sono molto avvicinati fra di loro e quasi si toccano.

Riferisco con altri a questa specie la Sphenopteris latifolia di Phillips, quantunque la figura ch' egli ne porge lasci molto a desiderare e non s'attagli perfettamente alla descrizione. Tuttavia esaminandola attentamente, mi sembra che le differenze ch'essa presenta in alcune sue parti debbansi riferire all' accidentale sovrapposizione di due saggi, l'uno spettante alla presente specie, l'altro a quella che segue.

Ciò che principalmente distingue la Cyclopteris digitata Brosa. dalle altre ed anche dalla Cyclopteris Huttoni di Sternberg che alcuni hanno ad essa riunita, si è il parallelismo delle divisioni dei lobi, la estremità tronca ed inciso-dentata dei medesimi ed il numero assai maggiore dei nervetti da cui è percorsa la fronda.

Questa specie si rinviene nelle arenarie e nelle argille scissili della serie oolitica inferiore tanto a Gristhorpe che a Cloughton presso Scarborough nell' Inghilterra.

\section{Cyclopteris Iuttoni Sternb.}

C. stipite tenui; fronde simplici profunde bi-tri-quinque-partita, in slipitem attenuala, partitionibus flabellato-cuneatis bi-tri-lobis, vel lanceolatis integris, lobis subinaequalibus, oblongis, obtusis, profunde emarginatis; nervis rarioribus, lenuibus, rectis, dichotomis, subparallelis.

Cyclopteris Ifuttoni Stennв. Vers. Flor. Vorw. II. pag. 66. - Göpr. Galt. Foss. Pflanz. 5-6, tab. 4. fig. 17-19. - Uxgen. Synops. pag. 53; Gen. et Spec. Plant. foss. pag. 94. - Zigso. Enum. Filic. foss. form. Oolith. pag. 22.

Cyclopteris digitata. Linde et Hutr. Foss. Flor. of Gr. Brit. I. pag. 179, Lab. 64. - DexKer. Monograph. d. Norddeutsch. Wealdenbild. pag. 9, tab. I. fig. 8. et 10,; tab. V. fig. 5. 6. (esclus. synon.) - Etrixash. Beitr. z. Flora d. Wealdenperiode, pag. 12, tab. IV. tig. 2. (exclus synon.).

Adiantites Huttoni Görp. Syst. Filic. foss. pag. 217.

Sphenopteris longifolia Beax. Pmilips. Geol. Yorksh. I. pag. 152, tab. 7. fig. 17.

In arenaria seriei oolithicae inferioris prope Scarborough Angliae. Juxta D. W. Dunker et Prof. von Ettingshausen occurrit etiam in formatione Wealdiana ad Obernkirchen, Duingen, Bückeburg, Deister et Osterwald Germaniae. 


\section{$-104-$}

Anche in questa specie lo stipite è piuttosto sottile e scanalato, e misura in larghezza due soli millimetri. La fronda semicircolare, nel suo generale contorno, giunge talvolta ad avere otto centimetri di larghezza e quattro di lunghezza. Essa è partita profondamente, fino al punto ove si fonde nello stipite, in due, tre e persino cinque divisioni un poco tra loro divaricate, che nella parte più ampia giungono talora ad un centimetro di larghezza, ed hanno forma flabellato-cuneata colle estremità, ora ottusamente lanceolate ed interissime, ora divise in due o tre lobi ineguali, bislunghi, ottusi 0 profondamente smarginati. I nervetti in questa specie sono meno avvicinati fra di loro, si dicotomizzano assai di rado e corrono ritti e paralleli fino all' estremità dei lobi.

Secondo il Sig. Leckenby trovasi nelle arenarie e nelle argille scissili sovrapposte alla Calcaria grigia della serie oolitica inferiore a Gristhorpe presso Scarborough in Inghilterra.

I Signori Dunker ed Ettingshausen ne figurarono parecchi esemplari trovati nella formazione Wealdiana in Germania ad Obernkirchen, Duingen, Bückeburg, Deister ed Osterwald.

Questa forma che lo Sternberg ed il Göppert avevano così bene distinta dalla $C y$ clopteris digitata del Brongniart, fu ad essa riunita dai Signori Dunker ed Ettingshausen senza farsi carico delle notabili differenze che presenta e che abbiamo cercato di far rilevare nelle descrizioni di queste due specie. Desta poi sorpresa come nella sinonimia abbiano annoverato anche la Bajera digitata di Braun, che cosi ricisamente se ne distingue, per le angustissime divisioni della fronda, che si dicotomizzano in lobi lineari colle estremità o tronche o semplicemente bidentate, e per la presenza di nervetti secondarii che si anastomizzano fra di loro dando origine ad una reticolazione a maglie assai allungate.

E rimarchevole come questa specie si trovi egualmente tanto nella serie oolitica dell'Inghilterra, quanto nella formazione dell' argilla di Weald della Germania. A dir vero, le figure che si osservano nell'opera del Dunker, essendo un po' confuse, darebbero forse adito a dubitare della identità della specie, ma quella bellissima che ci porge l'Ettingshausen (Beitr. z. Fl. Weald. Tav. IV. fig. 2.) vale a togliere ogni dubbio.

\section{Cyclopteris gracilis Zigno.}

C. stipite elongato, lineari, striato, supra canaliculato; fronde flabellata, profunde bipartita, in stipitem attenuata, partitionibus dichotomo-lobatis, 
lobis linearibus, elongatis, apice abrupte acuminatis; nervis simplicibus, parallelis, rarissime furcatis.

Cyclopteris gracilis. Zıко, Enum. Filic. foss. firm. Oolith. pag. 22.

Bajera? gracilis. Bunв. Foss. Plants of Scarb., in Quart. Journ. Soc. Gool. Lond. Vol. VII. pag. 182, tab. 12, fig. 3 .

Schizopteris gracilis. BEax, in mss.

In arenaria seriei oolithicae inferioris ad Cloughton prope Scarborough in Anglia.

Da uno stipite lineare assai allungato, ed avente un diametro di circa due millimetri, striato longitudinalmente e percorso nello stesso senso da un solco nella sua parte superiore, si distende gradatamente a ventaglio una fronda profondamente divisa in due parti, che alla lor volta si suddividono e dicotomizzano regolarmente fino a tre volte, ed i cui lobi terminali giungono ad una lunghezza di circa tre centimetri, e terminano rapidamente in punta. La fronda, verso la base, e poco solto alla sua partizione, ha una larghezza di due millimetri e mezzo ai tre, che si mantiene pressochè eguale nelle singole parti in cui è suddivisa, come pure fin verso l'estremità acuminata dei segmenti o lobi terminali, il cui apice finisce in punta un poco ottusa. I nervi che ne percorrono le varie parti per lo lungo sono poco numerosi, da tre a cinque soltanto, eguali, paralleli, per lo più semplici, rade volte forcuti, giammai confluenti, nè havi traccia alcuna di un nervo mediano più appariscente. In fine l'accurato esame delle figure e degli esemplari dà a divedere la disposizione dei nervi che è propria del genere a cui l'ho riferita.

Questa specie trovata nell' arenaria superiore della serie oolitica inferiore a Scarborough nell'Inghilterra è annoverata dal Sig. Leckenby fra le specie proprie del deposito di Cloughton.

Sir C. Bunbury, nel suo eccellente lavoro sulle piante fossili degli strati giuresi della contea di York, inserito nel fascicolo di Agosto dell' anno 1851, Vol. VII. del Quarterly Journal della Società Geologica di Londra, descrisse questa pianta, di cui ebbe ad osservare parecchi esemplari nelle collezioni dei Signori Murray, Phillips e Bean, e che quest' ultimo avea riferita al genere Schizopteris. Sir C. Bunbury facendo rilevare i caratteri differenziali che allontanano questa pianticella dal genere Schizopteris, la pone con un punto interrogativo nel genere Bajera, avvertendo come il Brongniart, nel suo Tableau des genres des Végétaux fossiles, favellando del genere Bajera, 


\section{$-106$}

avesse accennato alle analogie che presenta coll' aspetto generale della Cyclopteris Huttoni Stenns, e con quello di altra specie dell'oolite di Scarborough, a lobi lineari.

Osserva però il Brongniart, di non aver mai ravisato in queste due ultime forme, la reticolazione delle nervature secondarie, propria delle Bajere.

Anche Sir C. Bunbury dichiara mancare la sua specie di questo carattere, ed io pure ne riscontrai la mancanza negli esemplari, provenienti da Scarborough, da me posseduti, nei quali la distribuzione dei nervi si palesa eguale a quella delle altre specie del genere Cyclopteris; per cui mi sembra giustificato il suo collocamento in questo genere.

\section{Cyclopteris minor Zigno.}

Tav. IX. fig. 1. 1, a.

C. stipite rigidiusculo striato; fronde coriacea, transverse oblongo-rotundata, integerrima, superne depressa, lateraliter dilatata; nervis crassiusculis, e basi flabellatim adscendentibus, subflexuosis, aequalibus, bi-tri-furcatis.

Cyclopteris minor. Zigro, Enum. Filic. foss. form. Oolith. pag. 23.

In saxo calcareo ex stratis superioribus seriei oolithicae inferioris in Monte dicto Pernigotti, prope S. Bortolamio in Provincia Veronensi.

Da un picciuolo piuttosto consistente, e minutamente striato, di cui si scorge sul saggio una porzione lunga sei millimetri, ed avente il diametro di un solo millimetro, si espande la fronda di consistenza coriacea e di forma trasversalmente rotondato-bishunga, schiacciata all'apice e dilatata nei lati, lunga cinque millimetri e larga dieci, col margine superiore alcun poco sporgente. I nervi, avuto riguardo alla piccolezza della fronda, sono relativamente piuttosto robusti ed appariscenti. Essi sorgono dal picciuolo o stipite e scorrono disposti a ventaglio verso i margini della fronda, biforcandosi più volte e mostrandosi eguali fra di loro e leggermente flessuosi.

Trovai una sol volta questa specie nella calcaria scissile giallognola dell' oolite sul Monte Pernigotti presso S. Bortolamio nella Provincia di Verona.

Balzeranno all'occhio di oggnuno le analogie che passano fra la specie testè descritta e la Cyclopteris Brauniana del Chiarissimo Professore Göppert. Ed in vero stetti lungamente dubbioso se dovessi o no riferirla a quest' ultima specie. Se non che, oltre 


\section{$-107$}

alle dimensioni che sono di un terzo minori di quelle della specie Göppertiana, la forma trasversalmente bislunga, anzichè subreniforme, e quindi la mancanza dei profondi seni, che nella Cyclopteris Brauniana si osservano alla base della fronda lateralmente allo stipite, ed infine la sporgenza del margine superiore, che invece, nella $C$. Brauniana, ivi si mostra alquanto rientrante, m'indussero a considerarla quale specie diversa. Rimarrà poi a vedersi se questa forma, del pari che la C. Brauniana, ed altre che vi si avvicinano, rappresentino vere specie, o non siano piuttosto che lo stato giovane di qualche fronda non ancor sviluppata.

Devo qui far osservare che nell' ingrandimento delineato alla Tav. IX. fig. 1. $a$, il disegnatore non ha rappresentato i nervetti colla dovuta esattezza, avendo ommesso di segnarne la ripetuta biforcazione.

\section{Cyclopteris incisa Eichwald.}

C. fronde simplici lata, flabellato-semiorbiculari, incisa, multiloba, lobis primariis adnatis, incisuris superficialibus tantum distinctis, apicem versus discretis, bilobis, lobis rotundatis; nervis tenuibus aequalibus dicholomis.

Cyclopteris incisa. Eicnwaln, Lethaea Rossica. Vol. II. pag. 13, lav. IV. fig. 6.

In saxo calcareo ferruminoso formationis oulithicae Oxfordianae ad Kamenka, prope Izoume in Russia meridionali.

Nel saggio descritto e figurato dall' Eichwald manca lo stipite, di cui una lacerazione semicircolare, che si scorge alla base della fronda, segna soltanto il posto. La fronda è larga undici centimetri e due millimetri e lunga sei centimetri e mezzo. Essa è semplice di forma flabellata semiorbiculare, apparentemente partita in sei divisioni primarie, indicate da delle incisioni superficiali radianti dalla base, per cui le partizioni della fronda riescono invece congiunte fin verso l'apice ove si dividono l'una dall'altra e terminano partite in due lobi larghi e rotondati che non oltrepassano in lunghezza un centimetro. Le due laterali inferiori sono più anguste ed hanno all'incontro l'estremità intera e semplicemente ovato-ottusa. I nervetti assai sottili, numerosi ed eguali fra di loro, si dicotomizzano, scorrendo, in forma flabellata, verso i margini della fronda con lieve divergenza.

Questa specie fu trovata a Kamenka nei dintorni d'Izoume nella Russia meridionale, in un calcare ferruginoso che ritiensi appartenere al gruppo Oxfordiano della formazione Oolitica. 


\section{Cyclopteris lingua Eichw.}

C. fronde simplici? lanceolato-elongata, basi rolundata, apicem versus angustiore falcata, apice obtuso; nervis tenuissimis, approximalis, simplicibus, interdum furcatis, e basi flabellatim radiantibus ad apicem marginesque frondis excurrentibus; interstitiis transversim striato-plicatis.

Cyclopteris lingua. Eıcnwald, Lethaea Rossica. Vol. II. pag. 12, lav. II. fig. 4. $a, b$.

In eadem formatione ad Kamenka prope Izoume in Russia meridionali.

La fronda è lanceolata, allungata, rotondata inferiormente ed attenuata verso l'estremità, ove si curva da un lato a mo' di falce e finisce coll'apice ottuso. Essa misura in lunghezza sei centimetri e mezzo, e due nel punto della sua maggiore larghezza. I nervetti sottilissimi, assai avvicinati fra loro, ora semplici, ora biforcati, sorgono dalla base e si dirigono a guisa di ventaglio verso i margini laterali e l'estremità della fronda. Negli spazii frapposti ai nervi si osservano delle singolari pieghe o strie trasversali, che danno un aspetto reticolato alla nervatura (Vedi l'ingrandimento fig. $4 . \quad b$ della tav. II. nella sopra citata opera dell' Eichrvald.).

Anche questa specie proviene dal calcare ferruginoso di Kamenka presso Izoume nella Russia meridionale.

La forma che ci presenta questo saggio permette di sospettare che possa avere appartenuto piutlosto ad una fronda digitata o fors'anco pinnata, anzichè semplice quale ce la indica l'Eichwald.

In quanto poi alle strie trasversali figurate nell'ingrandimento (fig. 4. $b$ ), esse farebbero credere alla presenza di nervetti secondarii che congiungessero fra loro i nervi primarii, dando origine a delle areole quadrate che allontanerebbero ricisamente questa forma dal genere Cyclopteris, se l'autore della Lethaea Rossica non ci avesse fatto avvertiti che sono semplici piegature, probabilmente accidentali, dal che siamo indotti a concludere che non furono esattamente delineate nella citata figura, e che la loro regolarità e sporgenza vi sieno oltremodo esagerate. 


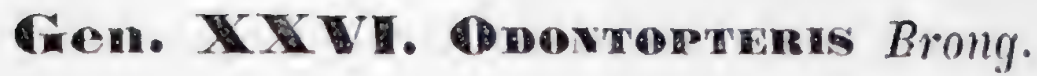

Frons pinnata vel bipinnata. Pinnae pinnulaeve, basi integra rachi adnatae. Nervus medius nullus vel vix notalus. Nervuli aequales, simplices aut furcati, tenuissimi, e rachide seriatim exorientes ad apicem marginemve pinnularum excurrentes.

Broxg. Prodrom. pag. 60 ; Hist. Végét. foss. 1. pag. 250 ; Tabl. des Gen. des Végét. foss. pag. 18. - Görp. Syst. Filic. foss. pag. 208; Gatl. foss. Pflanz. pag. 97. - Ura. Gen. et Spec. Plant. foss. pag. 88.

\section{Odlontopteris? jurensis Kurr.}

0. fronde coriacea bipinnata, rachide crassa squamosa; pinnis alternis, sessilibus, patulis, lanceolato-linearibus elongatis obtusis; pinnulis alternis, adnatis, conliguis, ovato-oblongis, obliquis, integerrimis, apice rotundatis, ad basim, longitudinaliter brevissime medio plicatis; nervis, nervulisque inconspicuis.

Odontopteris? jurensis. Kunn. Beitr. zur foss. Flor. a. Jura form. Würlemb. pag. 12, tab. I1. fig. 1. - Zısхo, Enum. Filic. foss. form. Oolith. pag. 23.

Invenit D.r Ferd. Krauss in calcaria coralliana formationis oolithicae, apud Schnaitheim in Germania.

Questo saggio, descritto e figurato dal Prof. Kurr, ci presenta una porzione di fronda bipinnata, lunga diecinove centimetri e larga all'incirca sette, sorretta da una rachide molto grossa e sporgente, coperta di piccole squamme, ed avente al basso un diametro di circa quattro millimetri, che assai lentamente si assottiglia procedendo verso l'estremità superiore della fronda. Escono dalla rachide primaria le pinne, alterne, di forma lineare lanceolata, allungata, ed ottuse all'apice. Esse sono larghe quindici millimetri, e lunghe dai tre ai quattro centimetri, e distanti l'una dall'altra nel punto d'inserzione dai dieci ai dodici millimetri. Le pinnule di cui sono fittamente fornite sono coriacee, alterne, contigue fra di loro, ed aderenti obbliquamente per tutta la base alle rachidi secondarie, di forma ovato-bislunga, interissime e rotondate nell'estremità, con una brevissima piega longitudinale nel mezzo della loro parte inferiore e prive di qualsiasi traccia di nervi. 
Questa specie fu trovata dal D.r Ferdinando Krauss nella calcaria del piano coralliano dell' oolite media, presso Schnaitheim nell'Alemagna.

La mancanza di ogni indizio della nervatura rende assai dubbia l'appartenenza di questa forma al genere Odontopteris, ed è soltanto dall'apparenza complessiva dei caratteri generali che si può essere indolti a collocarla temporaneamente in questo genere, fino a che il ritrovamento di qualche esemplare, munito dei nervi, venga a svelarci in qual gruppo generico debba realmente essere annoverata.

\section{Odontopteris Ungeri Zigno.}

Tav. XI. fig. 1, 2, 3, 3, a.

0. fronde coriacea bipinnata; pinnis pinnatifidis, sessilibus, approximatis, patentibus, lineari-lanceolatis elongatis-obtusis; pinnulis vel laciniis alternis, crassis, semiorbicularibus, oblongis, obliquis, integerrimis, confluentibus, terminali elongata subintegra tantum sinuato-crenata; nervis tenuibus, crebris, aequalibus, simplicibus, interdum furcatis, seriatim e rachide pinnarum exorientibus ad apicem marginemve pinnularum excurrentibus.

Odontopteris Ungeri. Zigso, Enum. Filic. foss. form. Oolith. pag. 23.

Cyeadopteris Ungeri. Zigno, Sulle Cicad. foss. dell' oolite in Riviste dell'Accademia di Padova 1853, pag. 349; Entdeck. foss. Pflanz. in d. Jura d. Venet. Alp. in Neu. Jahrb. Min. Geol. Palaeont.v. Leonh. u. Bronn. 1854, pag. 34.

In calcarea cinerea seriei oolithicae inferioris apud Rotzo in Provincia Vicentina, nec non in saxo calcareo lutescente vel cinereo fusco ad Montem Pernigotti in Provincia Veronensi.

La fronda di questa specie mostra una consistenza coriacea, ed in alcuni saggi svela una forma bipinnata. Le pinne, lunghe dagli otto agli undici centimetri, e larghe dai quindici ai venticinque millimetri, sono sessili, avvicinate, patenti, lineari-lanceolate allungate, coll'apice ottuso. Hanno forma pinnatifida colle lacinie o pinnule semicircolari, un po' bislunghe ed oblique, interissime, assai approssimate e congiunte fra di loro poco prima della base. Esse variano in lunghezza dai dieci ai quindici millimetri, ed in larghezza degli otto ai dieci. La pinnula terminale che forma l'estremità della pinna è assai più grande ed allungata ed apparisce composta dalla riunione di più lacinie contigue in tutta la loro lunghezza ed indicate soltanto dalle curve molto allargate che ne 


\section{$-111$}

rendono i margini in certa guisa sinuato-crenati. Mancano le lacinie di qualsiasi indizio di nervo mediano, ed inserite per tutta la base sulla rachide, si mostrano percorse da numerosi nervetti, esilissimi, or semplici, ora forcuti, che escono tutti egualmente dalla rachide, e scorrono, con lievissima divergenza, fino all' estremità, ed ai margini delle lacinie. Della rachide principale, che deve avere avuto una grossezza ragguardevole, non si può dai saggi fin qui trovati, formarsi una chiara idea, veggendosene soltanto qualche frammento striato longitudinalmente. Bensì scorgesi l'impressione lasciata dalle rachidi secondarie dalla quale rilevasi che aveano un diametro di circa due millimetri, e facevansi più sottili verso l'estremità, continuando fino all' apice della pinna.

I due esemplari figurati nella Tav. XI. fig. 1.2. furono trovati nella calcarea giallognola del Monte Pernigotti nel Veronese, e quello rappresentato dalla fig. 3. nella calcarea grigia di Rotzo nel Vicentino, roccie che tutte due appartengono allo slesso orizzonte geologico della serie oolitica inferiore.

Nel 1853 io aveva collocato questa forma nel mio genere Cycadopteris, tratto in errore da alcuni saggi malconci privi affatto di nervi, ma le cui pinnule coriacee ed in apparenza marginate mi sembravano autorizzare tale riunione. Però avendo di poi scoperto la nervatura pinnata delle vere Gycadopteris (V. Zrgno, Sopra un nnovo genere di Felce fossile. Alli dell'Istituto Veneto. Vol. VI. Serie III. 1861), ed essendomi imbattuto in qualche esemplare della presente specie, in cui erano ben visibili i nervi, riconobbi in essa, si per la forma ed inserzione delle pinnule, che per il numero e la disposizione dei nervetti da cui sono percorse, tale un' analogia colle Odontotteridi da ritenermi sufficientemente giustificato se la ripongo in quest'ultimo genere. ì bensi vero che la consistenza coriacea della fronda non è un carattere che si riscontri comunemente nelle Odontopteris, ma non lo credo bastante a separarnela, tanto più che anche la Odontopteris Jurensis del Kurr è pure indicata colla fronda coriacea.

\section{Ddontopteris? Leckenbyi Zigno.}

O. fronde bipinnata, rachide crassa profunde striato-sulcata; pinnis subopposilis, oblique patulis; pinnulis oppositis, basi dilatata rachidi adnatis, subcontiguis, integerrimis, oblongis, falcatis, apice angustalis incurvis, obtusis; nervis basi remoliusculis, e rachide seriatim exorientibus, ad apicem marginemque pinnularum excurentibus, subparallelis, bi-tri-furcatis.

Clenis Leckenbyi. Bens, MS. in Lecierbr, On the Sandst, and Shal. of Ool. of Scart. ete. in Quart. Journ. Soc. Geol. Lond. Vol. XX. Proceedings. pag. 78, tar. 10. fig. 1. 1, a. 1, 6. 


\section{$-112$}

In formatione oolithica ad Gristhorpe, prope Scarborough, in Comitatu York Angliae.

Questa specie ha una fronda bipinnata in cui la rachide principale, striata e profondamente solcata, appare piuttosto robusta e grossa, mentre le secondarie si palesano assai più sottili ed attenuate. Le pinne sono quasi opposte ed cscono obbliquamente dalla rachide primaria scostandosi poscia gradatamente e facendosi alquanto patenti. Esse sono fornite di pinnule opposte, colla base lievemente dilatata ed inserita per intero sulla rachide, ove al punto d'inserzione sembrano fra loro congiunte lateralmente pel lembo inferiore e separate da un seno rotondato più o meno angusto. Sono interissime, bislunghe e falciformi, lunghe circa ventiqualtro millimetri e larghe poco più di dieci; verso l'apice si stringono, si curvano all'insù, e terminano in punta assai ottusa. I nervetti che le percorrono sono poco numerosi (per lo più sei) ed escono dalla rachide a qualche distanza l'uno dall'altro, poi facendosi due o tre volte forcuti corrono quasi paralleli fino all' estremità ed ai margini delle pinnule.

L'esemplare figurato dal Signor Leckenby fu trovato nelle roccie della formazione oolitica a Gristhorpe presso Scarborough in Inghilterra, e la figura che abbiamo citata lo rappresenta ridotto a tre quarti della sua naturale grandezza.

Il Chiarissimo Sig. Bean collocò questa specie nel genere Gtenis, indotto probabilmente dal modo d'inserzione delle pinnule, dalla distanza, ehe verso la base separa l'uno dall'altro i nervetti che le pereorrono, e da una certa affiniti che traspare dall'aspetto generale delle pinne. Però, la forma bipinnata della fronda non si è finora riscontrata nel genere Ctenis, e ciò, non solo nell' unica specie, che secondo la mia opinione, veramente vi appartiene, e che è la Ctenis falcata Lrnd. IIutr., ma neppure in quelle che furono erroneamente annoverate in questo genere dal Braun, e che devono invece prender posto fra le Cicadeacee, mentre il genere Gtenis, distinto nella specie tipica, per la reticolazione dei nervetti secondarii, spetta senza dubbio alla classe delle Felci.

L'assenza di questa nervatura areolata, e la forma bipinnata della fronda, non permettono la collocazione della presente specie nel genere in cui fu posta dal Sig. Bean, mentre quest' ultimo carattere, congiunto al parallelismo dei nervetti che percorrono le pinnule, e che escono tutti egualmente dalla rachide, non si riscontra che nel genere Odontopteris. E bensi vero, che nelle Odontopteris, i nervetti sono assai più numerosi e più avvicinati, ma però questo è il solo genere cui, per il loro modo di distribuzione, possa riferirsi per ora la specie descritta e figurata dal Sig. Leckenby. 


\section{Gen. N⿴囗十. Dichopteris Zigno.}

Frons bipartita, bipinnata, rachide primaria, crassa, striata. Pinnae liberae, pinnatifidae, alternae vel suboppositae. Pinnulae coriaceae integerrimae, saepe basi angustatae, in rachides alatas decurrentes. Nervi aequales pauci, simplices, interdum furcati, e rachide seriatim orti, ad apicem marginemve pinnularum flabellatim excurrentes. Sori rotundi, prominuli, sparsi. Capsulae (Sporangia) ovato-globosae, sessiles, vel subsessiles, annulo completo cinctae.

Filices elasticae, rachide crassa bipartita, facie Gleicheniacearum.

Zıgno, Monogr. Gen. Dichopt. in Mem. Istit. Veneti. Vol. XII. pag. 217; Enum. Filic. foss. form. Oolith. pag. 23.

L'aspetto generale e costante che presenta questo genere, si è quello di due frondi bipinnate riunite per la base ad una rachide principale comune ad entrambe. Questa rachide assai grossa e consistente, il cui diametro, nell'estremità inferiore degli esemplari più grandi, arriva sovente ai due centimetri e mezzo, è segnata per lo lungo da numerose strie irregolari ed assai ravvicinate. Ad una certa distanza dalla base essa si parte in due soli rami che costituiscono le rachidi primarie delle due grandi divisioni della fronda e corrono ritli fino all'apice di cadauna, guerniti a brevi distanze da numerose pinne pinnatifide, alterne, lineari, allungate, per lo più patenti, talvolta obblique e percorse da una rachide secondaria striata e lateralmente alata, che s'ingrossa al punto d'inserzione sul ramo della rachide principale.

Le rachidi delle pinne sono fiancheggiate da pinnule interissime, patenti ed alterne, assai di rado quasi opposte, e generalmente un po' ristrette alla base ove sono decorrenti e connate in guisa da formar ala alla rachide. Esse sono fornite di pochi nervicciuoli eguali fra di loro, per lo più semplici, fra i quali se ne ravvisa qualche volta taluno forcuto o bipartito. Questi nervetti partono tutti dalla base della pinnula, e poscia lievemente divergendo e scostandosi l'uno dall'altro a forma di ventaglio vanno, senza curvarsi, a por fine al margine terminale della pinnula. La fruttificazione si presenta sotto l'aspetto di sori sporgenti dalla cuticola, di forma rotonda e sparsi irregolarmente per la pagina inferiore delle pinnule. Essi contengono degli sporangi di forma globosa tendente all'ovale, in cui finora non potei ravvisare alcuna traccia di pe- 
dicello, e che si mostrano cinti tult'all'intorno da un largo anello articolato a brevissimi intervalli. Gli sporangi fin qui osservati sono tutti aperti e vuoti, perciò non mi fil possibile chiarire la forma delle spore.

Il caraltere della divisione in due rami della rachide principale, da me riscontrato sopra gran numero di esemplari, essendo quello che balza all'occhio fin dalle prime, t più colpisce nell'esame di queste forme vegetali, m'indusse a distinguere questo genere col nome di Dichopteris, che significa Felce bipartita o doppia, perchè appunto presenta l'aspelto di due frondi di felce bipinnata riunite per la base. Ora ponendoci ad investigare le differenze che allontanano questo genere da tutti gli altri, tanto della Flora attuale, quanto di quelle che vegetavano nelle passate epoche geologiche, basterà accennare ai caratteri che lo distinguono da que' generi, i quali a prima giunta sembrerebbero ad esso più affini. Perciò dobbiamo anzi tutto osservare come nelle Felci viventi a fronda pinnatifida ed a sori rotondi e sparsi, non si riscontri la disposizione flabellata nei nervetti delle pinnule. Nelle Felci della Flora attuale, tale distribuzione dei nervi si osserva soltanto nei generi in cui la fronda è o semplice, o flabellata, o pinnato-composta, o sopradecomposta; mentre nelle Felci a fronda pinnatifida domina invece la nervatura pinnata o reticolata. Inoltre la fruttificazione, nelle Felci viventi a nervetti eguali e flabellati, si compone per lo più di sori lineari, rade volte di forma rotonda, che stan quasi sempre collocati lungo il margine della pinnula o disposti nel senso della nervatura, nè giammai si trovano sparsi su tutta la pagina inferiore, come nel genere presente. Da ciò ne consegue che la distribuzione dei nervetti, la disposizione dei sori e la bipartizione della fronda, ci presentano nel nostro genere tre caratteri, la cui riunione vale a costituirlo diverso da tutti quelli dell' epoca attuale.

Fra le Telci fossili troviamo il solo genere Odontopteris che al nostro si avvicini per un'analoga disposizione dei nervetli delle pinnule, congiunta alla forma pinnatifida delle pinne. Ma la frutlificazione delle Odontotteridi, quale ci fu indicata dal Göppert (Gatt. foss. Pflanz. Lief. 5. 6. pag. 98. tav. VI. fig. 1-5), e più recentemente dal Geinitz (Die Verstein. d. Steikohlenform. in Sachsen, pag. 21. Lav. XXVI. fig. 10-11), è affatto diversa e si palesa invece composta di vescichette rotondate che sostituiscono le pinnule nella parte terminale della fronda. Oltre a ciò i nervetti delle pinnule nelle Odontopteris sono assai più sottili e numerosi che nelle Dichopteris. All'incontro la consistenza della fronda, e la forma delle pinne e delle pinnule, presentano a primo aspetto qualche maggiore rassomiglianza coi generi Pachypteris del Brongniart, Thimnfeldia dell'Eungshausen e Kirchneria di Braun. Però, nelle Pachypteris, ogni pinnula è percorsa da un solo nervo mediano, nelle Thinnfeldie i nervetti delle pinnule sono più numerosi, più frequentemente forcuti, più riuniti verso la base, ove talvolta sembrano co- 
stituire per breve tratto un nervo mediano e poscia divergono curvandosi all'infuori in senso opposto, verso i margini delle pinnule. Nella Thimfeldia speciosa Etringsu sono poi decisamente pinnati ed hanno un nervo mediano bene marcato. Nelle Kirchnerie scorre in mezzo alle pinnule un nervo primario che verso l'apice si frastaglia in più nervicciuoli forcuti, e che è fiancheggiato da nervetti secondari obbliqui, uscenti dai suoi lati in forma pinnata, che si biforcano più volte verso i margini delle pinnule.

Ma oltre tutte queste differenze che distinguono questi generi dal nostro, havi la costante bipartizione della rachide primaria che to impronta di un carattere tutto suo. E bensi vero che il Chiarissimo Prof. Göppert definisce il suo genere Gleichenites colle parole Frons dichotoma pimnata; ma nelle Gleichenie viventi la dicotomia si estende anche alle pinne secondarie, mentre nelle Dichopteris si limita, come vedemmo, alla divisione della rachide principale in due rami forniti di pinne costantemente pinnatifide. Inoltre nelle Gleichenites di Göppert la nervatura delle pinnule è flabellato-pinnata, e nelle Gleichenie viventi è decisamente pinnata.

Il nostro genere adunque, per l'aspetto generale delle pinnule, ricorda le Pachypteris, le Kirchnerie e le Thimnfeldie, per la distribuzione dei nervetti s'approssima alle Odontopteris, e pella bipartizione della fronda alle Gleichenites di Göppert. Tultavia diversifica da ognuno di questi generi pei caratteri, a mio avviso, abbastanza distinti, di cui ho fatto superiormente parola.

Sette forme diverse riunisco nel genere Dichopteris distinguendole in altrettante specie basate principalmente sulla forma e sulle dimensioni delle pinnule, quante volte queste diversita non mi apparvero cagionate dalla semplice differenza di età. Cinque di queste specie si trovano nei terreni giurassici del Veneto, e due appartengono a quelli dell'Inghilterra, ed ebbero finora altre denominazioni. Di quest'ultime diede le figure il Prof. J. Phillips nella sua bell'Opera intitolata: Illustrations of the Geology of Yorkshire, ove appose il nome di Sphaenopteris lanceolata all' una (tav. X. fig. 6) e di Neuropteris laevigata all'altra (tav. X. fig. 9), e tutte due, tanto per la forma e disposizione delle pinnule, che per la nervatura onde sono percorse, corrispondono ai caratteri da me assegnati al genere Dichopteris.

L'illustre Brongniart nel suo Prodrome d'une Histoire des Végétaux fossiles, fondava fino dal 1828 il genere Pachypteris, e qualche anno dopo, nella Histoire des V'́gétaux fossiles, caratterizzandolo colla frase: Foliae pinnatae vel bipinnatae, pinmulis integris coriaceis enerviis vel uninerviis, basi constrictis nec rachi adnatis, collocava nella sinonimia della sua Pachypteris lanceolata, la Sphaenopteris lanceolata di Phillips, ed in quella della Pachypteris ovata, la Neuropteris laevigata del medesimo autore, accompagnando le descrizioni delle due specie con due figure (Hist. Végét. foss. 
1. pag. 166, Tav. 45, fig. 1.2.), che per nulla s'attagliano a quelle pubblicate dal Phillips, le quali io qui riproduco (Tav. XIV. fig. 2. 3.) per far vedere la diversa disposizione della nervatura in confronto di quella indicata nelle diagnosi e nelle figure del genere Brongniartiano.

E per vero, come potrebbonsi collocare nel genere Pachypteris, distinto colle pa, role: pimmlis enerviis vel uninerviis, due pianticelle che nelle loro pinnule palesano una nervatura composta di parecchi nervicciuoli tutti eguali e flabellati? Questa disposizione dei nervetti delle pinnule corrisponde perfettamente con quella di tutte le altre forme da me riunite nel genere Dichopteris, e si scosta egualmente tanto dalle Pachypteris del Brongniart, quanto dai generi Sphaenopteris e Neuropteris cui Phillips aveva con qualche dubbio riferite le sue due specie.

Per questi motivi, quando alla pag. 73 della presente Opera trattai delle Pachypteris, esclusi le due specie figurate dal Phillips, dalla sinonimia della Pachypteris lanceolata e della Pachypteris ovata del Brongniart, riservandomi di annoverarle fra le Dichopteris, come feci nella Monografia di questo genere, pubblicata nel 1865, nel Volume XII. delle Memorie del R. Istituto Veneto di Scienze, Lettere ed Arti alle pagine 223 e 224.

\section{Dichopteris Wisianica Zigno.}

Tav. XII. fig. 1. 1, a. Tav. XIII. fig. 1. 1, a. b.

D. fronde bipartita bipinnata; rachide crassissima striata constanter bifida; pinnis pinnatifidis, alternis, linearibus, elongatis, subpatulis apice attenuatis obtusis, rachidibus alatis, striatis, basi incrassatis; pinnulis coriaceis, suboppositis, patulis ovato-lanceolatis oblongis, apice subfalcatis, obtusis, basi vix constrictis confluentibus ac in rachidem decurrentibus, terminali exigua obovata, nervis $\mathbf{5}-\mathbf{6}$ flabellatis, interdum bifidis.

Dichopteris Visianica. Zigno, Monogr. Gen. Dichopteris in Mem. R. Istit. Venet. Vol. XII pag. 218, Tav. XI. fig. 1.2. 3.; Enum. Filic. foss. form. Oolith. pag. 25.

In stratis calcareae cinereae seriei oolithicae inferioris ad Rotzo et in Val d'Assa Provinciae Vicentinae, nec non ad Valles Juliani et Salaorno prope Roverè di Velo in Provincia Veronensi ubi magis abundat.

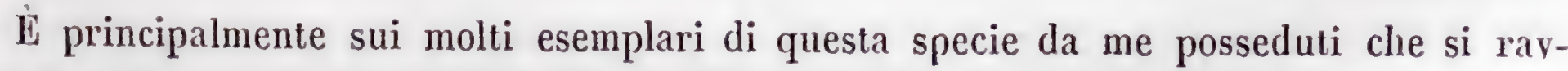
visa il carattere più importante fra quelli assegnati al genere, cioè la costante bipar- 


\section{$-117$}

tizione della rachide primaria. Il saggio di cui porgo la figura alla Tavola XIII. fig. 1. ridotta alla metà della sua naturale grandezza, è uno dei più belli e caratteristici ch' io possegga. Esso è lungo settanta centimetri, e ci presenta una rachide assai grossa e robusta, il cui diametro misura alla base due centimetri e mezzo, e va poi lentamente diminuendo, finchè dopo un tratto di ventisei centimetri si parte in due rami, cadauno della grossezza di otto in dieci millimetri, che gradatamente assottigliandosi, corrono rilti, con poca divergenza fino alle due estremità della fronda guerniti ai lati di numerose pinne. Però, anche prima dell'inforcatura della rachide principale, si veggono lateralmente uscire dal terzo superiore di essa, delle pinne affatto simili a quelle che rendono bipinnati i due rami procedenti dalla bipartizione della rachide primaria.

Uno di questi rami spettante ad altro saggio è delineato separatamente, nelle sue naturali dimensioni, alla Tav. XII. fig. 1.

Le pinne sono alterne, distanti fra loro circa due centimetri, e percorse da una rachide alata, striata, che al punto d'inserzione fortemente s'ingrossa a guisa di picciuolo. Esse sono pinnatifide, lineari lanceolate, allungate, gradatamente attenuate verso l'apice, e giungono spesso ad una larghezza di due centimetri ed alla lunghezza di dieciotto.

Le pinnule sono quasi opposte e patenti, di consistenza coriacea, di forma ovatolanceolata bislunga, coll'apice oltusamente falcato ed un po' obbliquo, lievemente ristrette alla base, per la quale sono unite fra di loro, e decorrono formando ala alla rachide. Esse hanno una lunghezza di circa dodici millimetri ed una larghezza di cinque o poco più; decrescono però gradatamente procedendo ver'so l'apice della pinna clıe è formato da una pinnula piccolissima ed obovata.

I nervetti che le percorrono partono tutti dalla base della pinnula e poi si allargano a ventaglio. Essi sono per lo più cinque o sei, eguali, semplici, rade volte forcati e di apparenza piuttosto rigida.

I saggi di questa specie provengono dalla calcarea grigia, della serie oolitica inferiore, che giace soltoposta al calcare ammonitico, e sono frequenti nella Valle di Salaorno e nella Valle Zuliani presso Roverè di Velo nella Provincia Veronese; trovansi pur anco, ma più di rado, a Rotzo e nella Val d'Assa, nei Sette Comuni del Vicentino.

La specie è dedicata all'illustre botanico e pregiatissimo mio amico, Prof. Cav. Roberto de Visiani, nella cui splendida collezione di piante fossili, se ne ammira pure un bellissimo esemplare. 


\section{$-118$}

\section{Dichopteris Ianceolata Zigno.}

Tav. XIV. fig. 2.

D. fronde pinnata vel bipinnata? rachide plana subalata; pinnulis inaequalibus obliquis, alternis, remotiusculis, obovato-lanceolatis, elongatis, obtusis, basi longe attenuatis, decurrentibus; nervis $\mathbf{3}-\mathbf{6}$ aequalibus flabellatis.

Dichopteris lanceolata. Zigro, Monogr. Gen. Dichopt. in Mem. R. Istit. Venet. Vol. XII. pag. 223, Tab. XIII. fig. 1.; Enum. Filic. foss. form. Oolith. pag. 24.

Sphaenopteris lanceolata. Punciss, Illustr. Geol. of Yorkshire. Vol. I. pag. 125, Tav. X. fig. 6.

Ad Saltwick in Comitatu York Angliae, in schisto argillaceo carbonifero formationis oolithicae.

La figura di questa specie, quale si osserva nella bella opera del Prof. Phillips intitolata: Illustrations of the Geology of Yorkshire. Vol. I. tav. X. fig. 6., ci presenta un frammento della lunghezza di otto centimetri, che per la sua posizione obbliqua permette sospettare che possa avere appartenuto ad una fronda bipartita e fors'anco bipinnata. Comunque ciò sia, corrisponde per tulti gli altri caratteri al nostro genere. La rachide apparisce piana, lıa un diametro di due millimetri ed è fornita di pinnule un poco irregolari nelle lor dimensioni, ma costantemente alterne, lanceolate, allungate, ottuse all' apice, attenuate verso la base, e decorrenti in guisa da formar ala alla rachide. Esse variano nella lunghezza dai quindici ai venti millimetri, e nella larghezza dai tre ai cinque, ed ognuna è fornita di cinque o sei nervetti eguali, flabellati, che corrono ritti, divergendo alcun poco, verso il margine terminale della pinnula.

Questo frammento fu rinvenuto negli schisti argillosi carboniferi della formazione oolitica a Saltwick nella Contea di York in Inghilterra.

\section{Diehopteris Iaevigata Zigno.}

Tav. XIV, fig. 3 .

D. fronde bipinnata, rachide alata, striata; pinnis alternis, remotis, patulis, lanceolato-linearibus, elongatis, pinnatifidis, apicem versus attenuatis; pinnulis vel laciniis brevibus, obovatis, apice rotundatis, basi angustatis, con- 
fluentibus opposilis, decurrentibus, terminali minima; rachidibus secundariis, tenuibus, alatis; nervis crebris, tenuissimis, aequalibus, flabellatis.

Dichopleris laevigata. Zısno, Monogr. Gen. Dichopt, in Mem. R. Istit. Venet. Vol. XII, pag. 224, lab. XIII. fig. 2.; Enum. Filic. foss. form. Oolith. pag. 24.

Neuropteris laevigata. Pniluirs, Illustr. Geol. of Yorksh. Vol. I. pag. 125, lav. X. fig. 9.

In eadem formatione ad Egton Moors et ad Haiburn Wyke prope Whitby in Gomitatu York, Angliae.

Il saggio figurato dal Phillips ci porge parte di una fronda bipinnata la cui rachide striata ed alata, sorpassa di poco in larghezza quella della specie precedente ed è munita di quattro pinne disposte alternamente, distanti l' una dall' altra circa dieciotto millimetri. Tre di queste mancano dell' estremità, una però si scorge intatta e palesa una forma lanceolato-lineare, avente alla base, ove è più larga, dai dodici ai quattordici millimetri di larghezza, e si ristringe poscia gradatamente procedendo verso l'apice. La lunghezza di questa pinna non oltrepassa i quattro centimetri. Le pinne sono percorse da una rachide assai sottile e si suddividono in forma pinnatifida colle pinnule o lacinie brevi opposte, obovate, assai rotondate all' apice e pochissimo attenuate alla base, ove sono decorrenti e connate.

Le pinnule maggiori collocate verso il punto d'inserzione delle pinne misurano dai sei agli otto millimetri di lunghezza, sopra tre o quattro di larghezza, mentre quelle poste verso l'estremità hanno due millimetri di larghezza sopra una lunghezza di tre, e la pinnula terminale è piccolissima. Ogni pinnula o lacinia è segnata da nervetti numerosi, minuti, semplici, disposti a ventaglio, che partono dalla base e si dirigono, con leggerissima divergenza, verso il margine della lacinia.

Anche questa specie deriva dagli schisti argillosi carboniferi della formazione oolitica d'Inghilterra e si rinvenne ad Egton Moors e ad Haiburn Wyke presso Whitby nella Contea di York.

Le descrizioni di queste due specie sono basate alle figure inviatemi dal Prof. Plitlips e che qui ripeto alla tav. XIV. fig. 2. 3., non avendo potuto procurarmene degli esemplari. Anzi sembra che i due saggi, i quali si conservano nel Museo della Società Filosofica di York, sieno gli unici fin qui trovati. 


\section{Dichopteris Paroliniana Zigno.}

Tav. XIV. fig. 4., Tav. XV. fig. 6.

D. fronde laevi pinnata vel bipinnata?; pinnis profunde pinnatifidis, lanceolato-elongatis; rachide gracili, subflexuosa alata; laciniis vel pinnulis obliquis, alternis, oblongis, cuneiformibus, apice oblique truncatis subfalcatis obtusis, sensim basim versus attenuatis, confluentibus, approximatis, decurrentibus; nervis tenuibus obsoletis.

Dichopteris Paroliniana. Zigno, Monogr. Gen. Dichopt. in Mem. R. Istit. Ven. Vol. XII. pag. 220 , tab. XIII. fig. 3.; Enum. Filic. foss. form. Oolith. pag. 24.

In calcarea cinerea formationis oolithicae ad Vallem Zuliani prope Roverè di Velo in Provincia Veronensi.

Da questo frammento di una sola pinna, non si può certamente decidere se la fronda di questa specie fosse bipinnata o semplicemente pinnata. Però, appoggiandosi a quanto si osserva nelle altre specie di questo genere, può ragionevolmente supporsi che anclie la fronda di questa fosse bipinnata.

Non si ravvisa in questa specie la consistenza coriacea delle altre, anzi sottili e quasi membranacee ci si palesano le pinne, e pure assai sottile e leggermente flessuosa è la rachide che percorre la pinna. Quest' ultima, nel suo contorno generale, ha una forma largamente lanceolata-allungata, che sembra terminasse ottusamente, e misura nella sua maggiore larghezza dai ventiotto ai trenta millimetri. La sua lunghezza non può determinarsi, poichè l'unico saggio, fin qui trovato, manca della base e di parte dell'estremità superiore.

Le pinnule, assai maggiori di quelle che si osservano in tutte le altre specie, giungono ad una lunghezza di circa dieciotto millimetri, sopra una larghezza di cinque nel punto ove sono più ampie, che è nel terzo superiore. Esse sono alterne ed hanno forma bislunga, cuneata verso la base ove, stringendosi gradatamente decorrono, e connate in senso verticale, vanno a formar l'ala della rachide. Verso l'estremità si allargano lentamente, finchè terminano coll'apice obbliquamente tronco, quasi falcato ed assai ottuso. Sono molto avvicinate fra di loro, alcune toccandosi nel punto della loro maggiore larghezza, e mostrano di essere segnate per lo lungo da nervicciuoli appena visibili ed assai più sottili di quelli che si scorgono nelle altre specie. Sulla superficie delle pinnule veggonsi pure le traccie di minutissime strie longitudinali. 


\section{$-121$}

Rinvenni questa specie nella calcarea grigia della serie oolitica inferiore in Valle Zuliani presso Roverè di Velo nella Provincia di Verona, e fino dal $1865 \mathrm{fu}$ da me intitolata al distinto naturalista e carissimo mio amico il Cav. Alberto Parolini di Bassano.

\section{Dichopteris angustifolia Zigno.}

Tav. XV. fig. 1.2.3

D. fronde coriacea, bipinnata; pinnis lineari-lanceolatis, pinnatis, apice attenuatis obtusis; rachide striata, subalata; pinnulis acqualibus alternis vel suboppositis, remotiusculis, linearibus, decurrentibus nec basi constrictis, apice subfalcatis, attenuatis, obtusis, tribus terminalibus brevissimis, media obtuse bidentata; nervis crassiusculis, in pinnulis inferioribus magis conspicuis.

Dichopteris angustifolia. Zigro, Monogr. Gen. Dichopt. in Mem. R. Istit. Ven. Vol. XII. pag. 222, tab. XIII. fig. 4. 5.; Enum. Filic. foss, form. Oolith. pag. 24.

In calcarea cinerea formationis oolithicae ad Vallem Zuliani prope Roverè di Velo in Provincia Veronensi.

La fronda di questa specie apparisce nei nostri saggi di consistenza coriacea e di forma bipinnata. Le pinne lineari, lanceolate, attenuate all' apice ed alquanto ottuse, sono larghe dai quindici ai venti millimetri e lunghe dagli otto ai nove centimetri. La rachide che la percorre è piuttosto consistente, striata e meno ampiamente alata, in confronto di quelle delle altre specie. Le pinnule (tav. XV. fig. 2.) sono quasi opposte, lineari, lunghe circa dodici millimetri, e larghe dai due ai tre, discoste appena due millimetri l'una dall'altra, eguali ed ottusamente appuntite. In qualche esemplare (tav. XV. fig. 3.) sono alterne e si mostrano leggermente falcate all'apice. Alla base non si contraggono e decorrono nella rachide in guisa che appena si può scorgere che vi formino ala. Le tre pimnulette che costituiscono l'estremità delle pinne sono brevissime, e quella posta nel mezzo è ritta, piccolissima, un poco più sporgente e bidentata all'apice. 1 nervetti sono poco visibili nelle pinnule superiori, ma nelle inferiori si palesano eguali a quelli della Dichopteris Visianica.

Tutti tre questi saggi provengono dalla calcarea ora grigia, ora giallo-bruna, spettante alla serie oolitica inferiore della Valle Zuliani presso Roverè di Velo nella Provincia di Verona. 


\section{Dichopteris rhomboidalis Zigno.}

Tav. XV. fig. 4 .

D. fronde laevi pinnata vel bipinnata, pinnis linearibus, rachide teretiuscula, striata, subalata; pinnulis membranaceis, alternis, patulis, ovatorhombeis, obtusis, basi constriclis, decurrentibus, superioribus obovatis; nervis tenuibus, fere omnibus obliteratis.

Dichopteris rhomboidalis. Zigno, Monogr. Gen. Dichopt. in Mem. R. Istit. Venet. Vol. XII. pag. 222 , lab. XIII. fig. 6 ; Enum. Filic. foss. form. Oolith. pag. 24

Eodem in loco, Provinciae Veronensis, cum praecedentibus.

Anche di questa specie abbiamo finora una sola pinna, e questa di forma lineare allungata, che va gradatamente stringendosi dalla base all' apice, misurando al basso due centimetri di larghezza e verso l'estremilà soli quattordici millimetri. Manca nel saggio porzione dell'apice, per cui non si può conoscere in qual modo avesse termine la pinna. La rachide da cui è sorretta, è sottile, e lungo il dorso della pinna scorgesi piuttosto rilevata e striata, mentre sulla faccia anteriore è marcata soltanto da una costa filiforme alata ed un po'flessuosa. Essa è fornita di pinnule alterne e patenti, di apparenza membranacea e di forma ovato-romboidale coll'apice ottuso, ristrette alla base e decorrenti, lunghe quindici millimetri e larghe sei. Nelle pinnule che sono presso all'estremità della pinna domina invece la forma obovata. Dei nervetti si scorge solo qualche traccia, la quale dà a divedere ch'erano assai sottili.

Questo saggio fu trovato con quelli delle specie precedenti nello stesso luogo e nel medesimo strato.

\section{Dichopteris mierophylla Zigno.}

Tav. XV. fig. 5, $a, b$.

D. fronde bipartita, bipinnata, rachide striato-sulcata; pinnis alternis, linearibus, apicem versus attenuatis, rachidibus alatis, profunde sulcatis, decurrentibus; pinnulis alternis, patulis, confertis subimbricatis, late linearibus oblongis, brevissimis, basi confluentibus, ac in rachidem decurren- 
tibus, terminali minima; nervis inconspicuis; soris rotundis, prominulis, irregulariter sparsis.

Dichopteris microphylla. Zıgno, Monogr. Gen. Dichopt. in Mem. R. Istit. Venet. Vol. XII. pag. 219, tab. XII. fig. 2.; Enum. Filic. foss. form. Oolith. pag. 24.

In calcarea cinereo-fusca formationis oolithicae ad Bienterle prope M. Alba nec non apud Roverè di Velo in Provincia Veronensi.

In questo esemplare non si può scorgere la bipartizione della rachide principale, però vi si osserva la parte superiore di uno dei due rami ed alcune pinne dell'altro ramo. Nel ramo visibile la rachide si palesa robusta, alquanto stretta e flessuosa, profondamente striato-solcata per lo lungo. Le pinne sono alterne, lineari, allungate, e misurano dagli otto ai dieci millimetri di larghezza presso la base, poi minuiscono lentamente procedendo verso l'estremità ove terminano ottusamente. La maggiore lunghezza a cui giungono le pinne sta fra $\mathrm{i}$ sei ed i sette centimetri. Sono profondamente pinnatifide, e le rachidi pure striato-solcate, da cui sono percorse, decorrono sul ramo della rachide primaria e riescono brevemente alate, per la confluenza delle pinnule, di cui sono foltamente guernite. Queste ultime sono alterne, patenti, molto avvicinate, per lo più toccandosi, e talvolta essendo embricate in guisa che il margine anteriore dell' una è ricoperto dal posteriore della successiva. La forma delle pinnule è largamente lineareoblunga, impercettibilmente un po' attenuata alla base e rotondata nell'estremità. Esse si distinguono per la loro picciolezza e brevità anche nel saggio figurato, che, per la sua grandezza, evvi ogni ragione di credere, fosse giunto al suo pieno accrescimento. Le maggiori non oltrepassano i cinque millimetri di lunghezza, sopra due e mezzo di larghezza. Al punto d'inserzione sono confluenti fra di loro e decorrono nella rachide. Nei saggi finora raccolti non potei ancora discernere la nervatura, ma non è a dubitarsi ch' essa ci si paleserà eguale nella disposizione a quella delle altre specie di questo genere. Bensì in un frammento di pinna trovato a Roverè di Velo mi fu dato accertarmi, che le piccole prominenze rotonde sparse qua e là sulle pinnule ne contenevano la fruttificazione. Poichè, avendo rivolto ogni mia cura ad esaminare il contenuto di queste prominenze, lacerandone la cuticola, e sottoponendo la materia che ne estrassi alle pratiche opportune per renderla trasparente, dopo molte prove, giunsi finalmente a discernere, in mezzo ad innumerevoli frammenti più o meno opachi ed informi, alcune vescichette trasparenti ovato-globose, senza traccia di pedicello, percorse da una fascia articolata, formante una specie di zona longitudinale, che cingeva tutta la vescichelta, fuorchè da un lato, ove mostravasi fessa nel senso trasversale ed aperta, precisamente come sogliono rimanere le capsule delle Felci dopo aver lasciato uscire le spore. 
La difficoltà che presenta la preparazione degli oggetti fossili non mi permise di poter scorgere più di sei di queste vescichette nelle molte indagini microscopiche da me istituite, ma queste però bastarono ad assicurarmi, che nelle prominenze osservate sulle pinnule si contenevano degli sporangi analoghi a quelli che riempiono i sori delle Felci viventi.

L' esemplare di cui porgo la figura fu estratto dalla calcaria grigio-oscura, sottoposta al calcare ammonitico, a Bienterle presso $M$. Alba, e qualche altro frammento munito dei sori fu trovato nella stessa roccia presso Roverè di Velo nel Veronese.

\section{Ordo XI. Neuropterideae.}

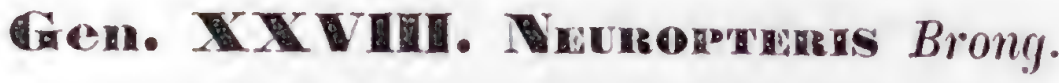

Frons pinnata vel bipinnata. Pinnae pinnulaeque basi cordatae vel subcordatae, integrae, liberae, rarius adnatae vel decurrentes. Nervus medius crassiusculus, apicem versus in nervulos dissolutus. Nervuli e nervo medio orientes, obliqui, arcuati, pluries furcati ad margines pinnularum usque producti. Fructificatio e pinnularum apice prostans, soris subrotundis, solitariis, exsertis.

Brong. Prodrom. pag. 52 ; Hist. Végét. foss. I. pag. 226 ; Tabl. Gen. Végét. foss. pag. 17 - Sterñ. Vers. Fl. Vorw. II. pag. 69. - Göpr. Syst. Filic. foss. pag. 173. - Scmimp. el Mougеот, Monogr. pag. 76. - Urg. Gen. el Spec. Plant. foss. pag. 74.

\section{Veuropteris undulata Lindl. Hutt.}

N. fronde bipinnata, rachide crassa sulcata; pinnis alternis, remotis, patenlissimis; pinnulis sessilibus, liberis, alternis, ovatis, basi cordatis, marginibus undulatis, infimis brevioribus obovatis, basi cuneatis; nervo medio tenui, apice evanescente; nervulis crebris approximatis, dichotomis, arcuatis.

Neuropteris undulata. Linde et Hutt. Foss. Flor. of Gr. Brit. II. tab. 83. - Sterne. Vers.

Fl. Vorw. II. pag. 137. - Zigvo, Enum. Filic. foss. form. Oolith. pag. 26.

Cheilanthites undulatus. Görp. Syst. Filic. foss. pag. 248. 


\section{$-125-$}

Sphenopteris undulata. Uxg. Gen. et Spec. Plant. foss. pag. 118.

Nolochlaena undulala. Eтrixgsm. Farrnkr. pag. 72.

In arenaria formationis oolithicae ad Gristorpe Bay prope Scarborough Angliae.

L'esemplare figurato dai Signori Lindley e Hutton manca delle estremità, per cui non si può indicare la forma generale della fronda. Vi si scorge una porzione della rachide, che nella parte più larga misura cinque millimetri, ed è percorsa per lo lungo da un solco profondo. Le pinne sono alterne, assai patenti, allungate, e distanti l'una dall'altra dai due centimetri e mezzo ai tre. Le rachidi secondarie sono assai sottili, non oltrepassando, col loro diametro, il millimetro, ma ingrossano a guisa di picciuolo al punto d'inserzione sulla rachide principale. Le pinnule di cui sono fornite sono sessili, alterne, di forma ovata un poco allungata, ottuse all'apice ed alquanto cordate alla base, col margine ondato tutto all'intorno. La loro maggiore lunghezza è di otto millimetri e nella parte più larga misurano quattro millimetri appena. Decrescono gradatamente procedendo verso la base delle pinne, ove sono assai più piccole misurando solo due millimetri di larghezza sopra una lunghezza di quattro. Ivi ne è pure diversa la forma, essendo più rotondate nell' estremità e stringendosi verso la base a guisa di cuneo. Il nervo mediano da cui sono percorse è assai sottile e svanisce presso l'apice della pinnula. I nervetti secondari sono numerosi, avvicinati fra di loro e forcuti. Essi escono obbliquamente dal nervo primario e divergono, curvandosi verso i margini laterali delle pinnule.

Questa specie fu rinvenuta dal Signor W. Williamson nelle arenarie e nelle marne della serie oolitica inferiore a Gristhorpe Bay presso Scarborough nell' Inghilterra.

Non saprebbesi, a dir vero, comprendere per qual ragione i Signori Lindley e Hutton abbiano riunita questa specie alla Neuropteris lobifolia di Phillips, quando nelle figure stesse che dell' una e dell'altra ci porgono (Foss. Fl. Gr. Brit. Vol. III. tav. 179, e Vol. II. tav. 83), balzano tosto all' occhio, non solo le differenze specifiche onde sono distinte, ma pur anco i caratteri che impediscono di annoverarle in uno stesso genere.

E per vero, solo che si ponga mente alla diversa forma delle pinnule, ed al loro differente modo d'inserzione sulla rachide, si scorgerà di leggieri come la presente specie debba prender posto fra le Neurotteridi e la Neuropteris lobifolia del Plillips fra le Pecotteridi. 


\section{Neuropteris arguta Lindl. Hutt.}

N. fronde bipinnata, rachide crassa striata; pinnis sessilibus, alternis, patulis, late linearibus elongatis, acuminatis; pinnulis, sterilibus, alternis, lineari-lanceolatis, subfalcatis acutis, approximatis, basi constrictis, in rachidem alatam breviter decurrentibus, marginibus crenulatis; fertilibus brevissimis, soris subrotundis solitariis, apice aculo pinnularum insidentibus, exsertis; nervo medio crassiusculo, apice evanescente; nervulis furcatis, obliquis arcuatis.

Neuropteris arguta. Linde. Hutr. Foss. Fl. Gr. Brit. Vol. Il. tab. 105. - Unger. Gen. et Spee, Plant. foss. pag. 88, --- Leciendx, On the Ool. of Scarb. with descrip. new spec. foss. Pl. in Quart. Journ. Geol. Soc. Lond. Vol. XX. Febr. 1864, pag. 74, 79, tab. X. fig. 4. - Zigro, Enum. Filic. foss. form. Oolith. pag. 26.

Pecopteris Lindleyana. Presl in Sternib. Vers. Fl. d. Worw. II. pag. 153. (non Royle).

Pleris Lindleyana. Eтtingsi. Farrnkr. pag. 114.

In eadem formatione ad Cloughton et ad Gristhorpe prope Scarborough Angliae.

La fronda bipinnata di questa specie, nel suo generale contorno, ci presenta una forma ampiamente lanceolata ed acuminata, percorsa da una rachide che ha un diametro di quattro millimetri all'incirca, finamente striata e fornita di pinne sessili, alterne, patenti, largamente lineari allungate, acuminate all' apice, che a prima vista sembrerebbero piuttosto profondamente pinnatifide, che pinnate. Se non che le pinnule, quantunque assai avvicinate decorrano brevemente nell'ala delle rachidi secondarie, pure sono fra loro disgiunte fino alla base, ed anzi si fanno più anguste presso al punto d'inserzione. Esse sono alterne, lineari-lanceolate, coi margini ottusamente crenati, un po' falcate ed acute all' apice. Le pinnule munite della fruttificazione, sono brevissime e quasi triangolari, aderenti per un lato alla rachide, mentre l'angolo opposto ne forma l'estremità sulla quale sporge un piccolo soro rotondo. Il nervo mediano è alquanto rilevato, ma svánisce verso l'apice dividendosi in ramoscelli forcuti; i nervetti secondari escono obbliquamente dal nervo primario, e dicotomizzandosi divergono ad arco verso $\mathrm{i}$ margini delle pinnule.

Questa specie si trova nelle argille della formazione oolitica tanto a Cloughton che a Gristhorpe Bay presso Scarborough nell' Inghilterra. 


\section{- $127-$}

Saggi forniti per intero di pinne fertili si erano trovati anche per lo addietro dal Signor Bean, ma credevasi che tal forma spettasse ad un nuovo genere di Felce. La scoperta fatta recentemente dal Signor Leckenby di un esemplare su cui si scorgono riunite le pinne fertili colle sterili, pose in chiaro come quella forma rappresenti invece la fruttificazione di questa specie. (Vedi Leckenby loc. cit. pag. 79, tav. X. fig. 4.)

\section{Ordo XII. Pecopterideae.}

\section{Gen. XXX. Hecopteris Brong.}

Frons pinnata, vel bi-tripinnata, aut bi-tripinnatifida. Pinnae lineares elongatae. Pinnulae basi aequali vel dilatata rachi adnatae, inter se unitae, rarissime basi contractae. Nervus medius valde notatus, subflexuosus passim apicem versus decrescens. Nervuli e nervo medio sub angulo recto vel acuto egredientes, raro simplices, saepius dichotomi; ramulis simplicibus vel furcatis, magis minusve arcuatim adscendentibus. Sori rotundi biseriales.

Brosg. Prodrom. pag. 54 ; Hist. Végét. foss. I. pag. 267; Tabl. Gen. Végét. foss. pag. 24. - Sterna. Vers. Fl. Vorw. II. pag. 146. - Unger. Synops. pag. 95; Gen. el Spec. Plant. foss. pag. 169.

Alethopteris. Sterna. Görp. Syst. Filic. foss. pag. 294. (pro parte)

Aspidites. Görr. Syst. Filic. foss. pag. 348. (pro parte)

Cladophlebis. Brong. Tabl. Gen. Végét. foss. pag. 25.

\section{Pecopteris recentior Phillips.}

P. fronde bipinnata, tripinnata (?); rachide crassa longitudinaliter sulcata; pinnis suboppositis, sessilibus, linearibus elongatis, patulis, rachidibus secundariis striatis; pinnulis oppositis alternisque, contiguis, rachi adnatis, sinu subrotundo discretis, horizontaliter patentibus, oblongis, subfalcatis obtusiusculis, integerrimis, laeviter incurvis, basi superne dilatata; nervo medio crassiusculo, apicem versus attenuato; nervulis tenuibus, obliquis, dichotomis, arcuatis. 


\section{$-128$}

Pecopteris recentior. Pniluss. Geol. of Yorksh. I. pag. 119, tab. VIII. fig. 15. (Ic. mala.) Boxbunx. On the Genus Neuropt. etc. in Quart. Journ. Geol. Soc. Lond. Vol. XIV. P. 3. pag. 246. - Zıso. Enum. Filic. foss. form. Oolith. pag. 26.

Neuropteris recentior. Lind. Hutt. Foss. Fl. Gr. Brit. I. pag. 195, lab. 68. - Stensn. Vers. Fl. Vorw. II. pag. 76. - Göre. Syst. Filic. foss. pag. 205. - Ung, Gen. et Spec. Plant. foss. pag. 85.

Cladophlebis recentior. Brosg. Tabl. Gen. Végét. foss. pag. 105.

Pteris recentior. Eтtixgsu. Farmlir. pag. 113.

In arenaria maryacea scissili seriei oolithicae inferioris ad Cloughton et ad Gristhorpe prope Scarborough in comitatu York, Angliae.

Dai grandi frammenti di fronda bipinnata che si conoscono, puossi ragionevolmente dedurre, che questa specie raggiungesse dimensioni ragguardevoli, e si dividesse for's' anco in forma tripinnata: la rachide principale si mostra piuttosto grossa e robusta, giungendo in alcuni esemplari al diametro di nove millimetri, ed è segnata longitudinalmente da parecchi solchi, continui e paralleli, che la dividono in un numero di coste convesse, appianate, e larghe all'incirca due millimetri. Naturalmente verso l'estrenità della fronda queste proporzioni si fanno minori. Le pinne sono sessili, quasi opposte, lineari, larghe, a seconda dell' età, dai quindici ai venticinque millimetri, e lunghe, a quanto sembra, oltre i venti centimetri. Esse sono percorse da una rachide striata, avente un diametro d'un millimetro e mezzo all'incirca, la quale decorre alcun poco sulla rachide principale. Le pinnule della parte superiore della fronda sono per la maggior parte opposte, le altre invece si mostrano alterne. Esse sono contigue e separate soltanto da seni rotondi più o meno angusti, e variano nella lunghezza dai cinque ai quindici millimetri; però al punto d'inserzione la loro larghezza si mantiene sempre fra i quattro ed i cinque millimetri. Ivi aderiscono per tutta la base alla rachide, ed il lembo superiore della base è dilatato, e forma ala alla rachide, mentre l'inferiore si stringe al punto d'inserzione per modo, che il nervo mediano riesce eccentrico e più approssimato al margine inferiore della pinnula che al superiore. La loro forma sarebbe lineare, lanceolata, bislunga, coll' apice piuttosto ottuso, ma la dilatazione superiore della base, congiunta alla curva generale della pinnula, le rende invece falcate. Il nervo mediano è abbastanza rilevato, e marcato fino all'estremità, verso la quale s'attenua. I nervetti secondari sono pure ben marcati, ma finissimi, obbliqui, dicotomi ed arcuati.

Da questa descrizione, che risulta dall' accurato esame degli esemplari, si scorgerà di leggieri, come le figure pubblicate dal Prof. Phillips e dai Signori Lindley ed Hutton, non rappresentino colla dovuta esattezza questa bella specie. In quella del Phillips non 


\section{$-129$}

è segnata la curva generale delle pinnule, ed in quella della Fossil Flora of Great Britain, è ommessa la dilatazione del lembo superiore della base; in tutte e due poi, le estremità sono molto più ottuse di quello lo sieno in realtà.

Questa specie rinviensi nell' arenaria scissile della serie oolitica inferiore, tanto a Gristhorpe quanto a Cloughton, presso Scarborough, , nell' Inghilterra.

L'illustre Brongniart non fece menzione di questa specie, nella sua Histoire des Végétaux fossiles, bensi la annovera nel suo Tableaux des Genres des Végétaux fossiles, fra le piante dell'oolite, sotto il nome di Cladophlebis recentior, cosi collocandola nella sua Sezione delle Pecotteridi Neurotteroidi che forma passaggio fra questi due generi. Mi sembra però, che il modo d'inserzione delle pinnule e la disposizione dei nervi, autorizzino a ritenerla nel genere Pecopteris, a cui fin dalle prime fu riferita dal Phillips, e tale vediamo pure essere l'opinione del chiarissimo Sir C. Bunbury (Vedi, On a remark. Spec. of Neuropt. with Remarlis on the Genus. in Quart. Journ. Geol. Soc. Lond. Vol. XIV. P. 3. pag. 246.).

\section{Cecoptenis denticulata Brong.}

P. fronde bipinnata, rachide angusta; pinnis sessilibus, alternis, approximatis, patulis, elongatis, profunde pinnatifidis; laciniis vel pinnulis adnalis obliquis, fere usque ad basim discrelis, alternis vel suboppositis, oblongo lanceolatis, falcatis, apice acuminatis, argute denticulatis, superioribus subtriangularibus arcuatis, sinu obtuso interstinclis; nervo medio recto, prominulo, apicem versus, attenuato; nervis secundariis e nervo medio oblique egredientibus, prope basim furcatis, ramulis rectis subparallelis.

Pecopteris denticulata. Bnong. Prodrom, pag. 57 ; Hist. Végét. foss. 1. pag. 30, tab. 98. fig. 1. 2. (esclus. synon. Phillips.) - Zıso. Enum. Filic. foss. form. Oolith. pag. 27

Neuropteris ligata. Lixd. IIvtr. Foss. Fl. Gr. Bril. Vol. I. pag. 197, Lab. 69. fig. 1. 2. (exclus. synon. Phillips.) - Sterki. Vers. Fl. Vorw. II. pag. 76. - Göpr. Syst. Filic. foss. pag. 205. -- Unger. Gen. et Spec. Plant. foss. pag. 86.

Pteris ligata. Eтtuxgsu. Furrnkr. pag. 113.

In arenaria formationis oolithicae ad Gristhorpe et ad Cloughton prope Scarborough Angliae. 


\section{$-130$}

La fronda bipinnata di questa bella specie, per quanto si può desumere dai saggi esistenti, deve aver raggiunta una larghezza di circa trenta centimetri, e si scorge percorsa da una rachide lievemente striata, che nel diametro non oltrepassa i quattro millimetri, e la cui sottigliezza piuttosto si attaglia alle dimensioni di una rachide secondaria, che a quelle di una rachide primaria; per il che riesce assai probabile l'opinione posta innanzi dai Signori Lindley e Hutton, che la fronda fosse anche tripinnata. Le pinne sono sessili, alterne, distanti l'una dall'altra, al punto d'inserzione, dai trenta ai trentacinque millimetri e sorrette da una rachide ritta, alquanto compressa e pure leggermente striata, che ha circa due millimetri di diametro verso la base delle pinne maggiori. Misurano quest'ultime tre centimetri e mezzo in larghezza, e dai frammenti raccolti, si può arguire che giungessero ad una lunghezza di quattordici centimetri all'incirca. Esse sono largamente lineari, allungate, piuttosto patenti, profondamente pinnatifide ed avvicinate in guisa, che si toccano colle estremità delle rispettive lacinie. Le pinnule o lacinie, ora alterne, ora quasi opposte ed alquanto obblique, quantunque assai fra loro vicine, sono però divise fin verso alla base, ove sono contigue. Hanno forma lanceolata, bislunga, falcata ed acuminata, col margine tutto all'intorno denticolato o, per meglio dire, minutamente seghettato. Raggiungono sovente una lunghezza di oltre due centimetri, e misurano nella parte più larga dai sei agli otto millimetri. Quelle che appartengono alle parti superiori della fronda sono invece quasi triangolari, arcuate, maggiormente acuminate ed un poco più discoste l'una dall'altra, per cui riescono separate verso la base da un seno piuttosto ottuso o rotondato. Il nervo mediano che le percorre va ritto e rilevato fin verso l'apice della pinnula ove s'attenua, ed i nervetti secondarii escono obbliquamente da quello e si dividono, poco sopra la loro origine, in due ramoscelli, che ritli e paralleli vanno a metter capo, con lieve divergenza e con direzione obbliqua, nelle addentellature del margine.

Questa specie si rinviene nell'argilla scissile spettante alla serie oolitica inferiore tanto a Gristhorpe che a Cloughton presso Scarborough nell'Inghilterra.

Ho escluso dalla sinonimia di questa specie la Pecopteris ligata di Phillips, quantunque sia ad essa riferita da tutti gli autori che ne diedero la descrizione, ad onta che lo stesso Phillips, l'avesse invece considerata quale sinonimo della Pecopteris Whitbiensis dei Signori Lindley e Hutton (Vedi Phillips, Geol. Yorksh. I. pag. 153). E per vero la figura del Phillips ci presenta delle pinnule molto più lentamente acuminate, col margine interissimo anzichè denticolato, e fornite di nervi secondarii più divergenti e quasi arcuati; e queste notevoli differenze mi parvero sufficienti a giustificare la sua esclusione dalla sinonimia della presente specie. 


\section{$-131$}

Il Professore Unger all'incontro pone la specie testè descritta fra i sinonimi della Pecopteris dentata L. H. che io descrivo più innanzi sotto il nome datole da Presl di Pecopteris Huttoniana, e che si distingue dalla Pecopteris denticulata Broś., per avere le pinnule più piccole, meno acuminate, seghettate soltanto verso l'apice, ed i nervi meno numerosi e più rilevati.

\section{Pecopteris lobifolia Lindl. Hutt.}

P. fronde bipinnata; rachidibus canaliculatis; pinnis alternis, patulis, linearibus elongatis, oblongis, profunde pinnatifidis; laciniis vel pinnulis ovato-lanceolatis obtusiusculis subfalcatis, basi vix constrictis, subdecurrentibus approximatis, sinu aculo oblique interstinctis, infimis inferioribus rachi proximis, obovatis, latioribus, obtuse $\mathbf{2}$-trilobatis, lobis inaequalibus, brevissimis, rotundatis; nervo medio tenui, subflexuoso; nervulis obliquis, crebris, tenuissimis, bifurcatis, arcuatis.

Pecopteris lobifolia. Linde. Hutr. Foss. Fl. Gr. Bril. III. pag. 79, tab. 179, tig. 1. 2. A (exclus. synon. Neuropteridis undulatae). - Stensi. Vers. Fl. Vorw. II. pag. 153. - Unger, Synops. pag. 99. - Zigxo. Enum. Filic. foss. form. Oolith. pag. 27.

Neuropteris lobifolia. Pmlurs. Geol. Yorksh. I. pag. 119, tab. 8. fig. 3.-Göpr. Syst. Filic. foss. pag. 206. - Unger. Gen. et Spec. Plant. foss. pag. 86.

Cladophlebis lobifolia. Brosa. Tabl. Gen. Végét. foss, pag. 105.

In eadem formatione ad Gristhorpe et Cloughton Angliae cum praecedentibus.

Questa specie ha una fronda bipinnata di aspetto piuttosto irregolare percorsa da una rachide scanalata avente il diametro di due millimetri. Le pinne sono pinnatifide, alterne, patenti, lineari, oblunghe, larghe circa venti millimetri, ma si attenuano verso la base, ivi riducendosi a soli dieci millimetri di larghezza. Nulla può dirsi circa la loro lunghezza, poichè tanto negli esemplari che ho osservato, quanto nelle figure date dal Phillips e dagli autori della Flora Fossile della Gran Bretagna mancano le estremità delle pinne. Le rachidi secondarie che le sorreggono sono piuttosto sottili, ma scorgonsi pure evidentemente segnate da una scanalatura longitudinale. Le pinnule o lacinie, lunghe dodici millimetri e larghe verso la base quattro millimetri all'incirca, hanno forma ovata, lanceolata, un po' falcata ed attenuata all'apice, ove terminano oltusamente. Verso la base si dilatano da prima alquanto, specialmente nel lembo superiore, cosicchè pajono quasi auricolate, poi si fanno insensibilmente piu anguste, ed inserendosi con tutta la 


\section{$-152$}

base obbliquamente sulla rachide appariscono decorrenti; esse sono assai avvicinate e solo divise da un seno acuto che per la sporgenza dell'orecchietta superiore della base, prende una direzione obbliqua. Decrescono nelle dimensioni procedendo verso il punto d'inserzione delle pinne, ma la ultima inferiore che si accolla alla rachide principale, scorgesi più grande, più larga, di forma obovala, divisa ottusamente in due o tre lobi ineguali, brevissimi e rotondati nelle estremità. Il nervo primario che le percorre è assai sottile ed alquanto flessuoso; i nervetti secondarj che da quello si diramano obbliquamente, sono tenuissimi, numerosi, due volte forcuti e lievemente arcuati.

Anche questa specie si trova colle precedenti tanto a Gristhorpe che a Cloughton presso Scarborough in Inghilterra, nello stesso terreno.

Diligenti confronti istituiti fra gli esemplari che mi pervennero dall' Inghilterra e le figure pubblicate dagli autori che ho citato, mi posero nella necessiti di rifondere e modificare le descrizioni anteriori della presente specie, e di ritenerla in pari tempo differente dalla Neuropteris undulata. Lisdu. Hutr., cosi confermando l'opinione espressa dal Presl, dal Göppert e dall'Unger che descrissero separatamente queste due forme, collocandole in generi diversi.

Il più volte citato, chiarissimo Signor Leckenby, sospetta che la forma figurata dai Signori Lindley e Itutton solto il nome di Tympanophora simplex. Foss. Fl. Gr. Brit. Vol. III. tav. 170. A. possa essere lo stato fertile della Pecopteris lobifolia L. H. Però dichiara di non essere ancor giunto ad averne il pieno convincimento, ed è per tal ragione che, altendendo i risultamenti delle ulteriori sue investigazioni, mi limito a far cenno di questa sua opinione, tralasciando per ora di comprendere questa forma nella descrizione della specie.

\section{Decopteris serrata Ung.}

P. fronde bipinnata, rachide sulcato-carinata; pinnis alternis, obliquis, remotis, elongatis; pinnulis linearibus, opposilis alternisque, patentibus, serrato-pinnatifidis, apice attenualis; laciniis subaequalibus alternis integerrimis, ovato-lanceolatis acutis, basi contiguis; nervis in qualibet lacinia pinnatis, rectis, excurrentibus; nervulis obliquis, simplicibus.

Pecopteris serrala. Unger. Synops. pag. 97; Gen. el Spec. Planl. foss. pag. 172. - Broxi. Tabl. Gen. Végét. foss, pag. 105. - Zigso. Enum. Filic. foss. form. Oolith. pag. 27. 


\section{$-153$}

Sphenopteris serrala. Linde. Ilutw. Foss. Fl. Gr. Brit. Vol. II. pag. 187, lab. 148. -- Sterni. Vers. Fl. Vorw. II. pag. 130.

Aspidites serratus. Görp. Syst. Filic. foss. pag. 363.

In arenaria ferruminosa seriei oolithicae inferioris ad Cloughton-Wyke prope Scarborough, Angliae.

La fronda di questa specie dir si potrebbe tripinnata a cagione della partizione quasi pinnatifida delle pinnule. Essa è percorsa da una rachide primaria solcato-carenata, avente il diametro di due millimetri e mezzo, e che s'attenua verso l'estremita, ivi diventando flessuosa. Le pinne, alcune delle quali oltrepassano i tredici centimetri di lunghezza sopra una larghezza di tre, sono alterne, diștanti l'una dall'altra circa tre centimetri ed inserite obbliquamente sulla rachide principale. Le rachidi secondarie da cui sono percorse, sono erette, leggermente flessuose, ed hanno un millimetro di diametro, e veggonsi regolarmente fornite di circa ventiquattro paja di pinnule che distano fra loro al punto d'inserzione, poco più di tre millimetri. Le dette pinnule sono per la maggior parte opposte e patenti, però verso le estremità delle pinne scorgonsi alterne ed alquanto oblique. Esse hamno una lunghezza che sta fra i dodici ed i dieciotto millimetri, e verso la base una costante larghezza di tre millimetri. La loro forma è lineare e lievemente s'attenua verso l'apice, e sono cosi profondamente seghettate da sembrar pinnatifide, colle lacinie quasi tutte eguali ovato-lanceolate, interissime, acute nell' estremità, dilatate alla base, contigue nel terzo inferiore, e decrescenti lentamente verso l'apice della pinna che riesce angusto ma non acuminato. I nervi sono pinnati, e dai nervetti principali che scorrono dritti nel mezzo delle lacinie fino all' apice, escono in forma pure pinnata i nervetti secondarj semplici ed obbliqui.

L'esemplare figurato nell'opera dei Signori Lindley e Hutton fu rinvenuto dal Signor Williamson a Cloughton-Wyke in un' arenaria ferruginosa a grossi elementi spettante alla serie oolitica inferiore.

\section{Decopteris Huttoniana Presl.}

P. fronde bipinnata, rachide crassa, medio sulcata; pinnis alternis, elongatis, patentibus, linearibus acuminatis, superioribus oppositis; pinnulis alternis approximalis ovato-lanceolatis acutis, falcatis, basi superne dilatata vel subauriculata, inferieribus apicem versus argute serratis, summis inte- 
gerrimis; nervo medio excurrente prominulo apice furcato, secundariis obliquis $\mathbf{1}$-bifurcatis subdivergentibus, ramulis erectiusculis.

Pecopteris Hultoniana. Presr, in Stennв. Vers. Fl. Vorw. II. pag. 157.

Pecopteris denlata. Willanson, in Linde. Hutr. Foss. Fl Gr. Brit. III. pag. 55, tab. 169. (non P. dentata. Bnovg. Hist. Végét. foss. I. pag. 346.)

Alethopteris dentala. Görp. Syst. Filic. foss. pag. 306. - Ung. Synops, pag. 82 ; Gen. et Spec. Plant. foss. pag. 149. (exclus. synon. Pecopteridis Brongniartianae Stennв., et P. denticulatae Broxg.)

Cladophlebis dentala. Brosg. Tabl. Gen. Végét. foss, pag. 105.

In arenaria inferiori formationis oolithicae ad Cloughton et ad Gristhorpe Bay prope Scarborough, in Anglia.

La fronda bipinnata ha una rachide del diametro di cinque millimetri percorsa nella sua faccia anteriore da un solco longitudinale poco profondo. Le pinne sono alterne, molto allungate, lineari, pinnate ed acuminate verso l'apice; quelle che stanno presso all' estremità della fronda sono invece opposte. Nel punto più largo misurano dai quindici ai dieciotto millimetri. In quanto alla lunghezza non posso dare precise indicazioni non avendo vedute pinne complete; dirò soltanto che in alcuni esemplari da me posseduti si scorgono delle porzioni di pinna della lunghezza di otto o dieci centimetri, che ancora non danno a divedere alcun principio di quel ristringimento che incomincia assai prima dell'estremità, e procedendo gradatamente le rende acuminate, il che manifesta esservi ancora una ragguardevole distanza da quel punto al termine della pinna. Le pinnule sono alterne ed assai avvicinate, però disgiunte fino alla base, ciò clee non è abbastanza chiaramente indicato nella figura data dai Signori Lindley e Hutton. Sono lunghe dai sei agli otto millimetri, e larghe dai tre ai cinque. La forma ne è ovato lanceolata, acuta e curva a guisa di falce, col lembo superiore della base dilatato in forma d'orecchietta. Quelle delle pinne inferiori sono verso l'apice finamente seghettate, nelle pinne superiori si palesano invece interissime. Questa specie si distingue per avere i nervi tutti molto marcati ed alquanto sporgenti. Il nervo principale, che per la dilatazione della pinnula nella parte superiore della base, ivi sorge più d'appresso al margine inferiore, scorre poi nel mezzo fino all' apice della pinnula ove si fa forcuto, ed i nervetti secondarii che escono dal primario obbliquamente sono alcun poco divergenti, specialmente quelli più prossimi alla base, e si mostrano una o due volte forcuti coi ramoscelli piuttosto eretti. 
Gli esemplari di questa specie si trovano frequentemente nelle arenarie e nelle argille inferiori della formazione oolitica tanto a Cloughton che a Gristhorpe Bay presso scarborough nell' Inghilterra.

I Professori Göppert ed Unger descrissero la specie di cui si tratta sotto il nome di Alethopteris dentata, ma conviene escludere dalla sinonimia appostavi dall' Unger, la Pecopteris Brongniartiana di Presl, e la Pecopteris denticulata del Brongniart. Imperciocchè la prima, che è la Pecopteris dentata Brosg. è tutt'altra specie, e si rinviene nella formazione carbonifera, e la seconda, ch'io descrissi più sopra, conservando il nome datole dal Brongniart, sebbene presenti qualche analogia colla presente, pure ne diversifica pei caratteri specialissimi che ho indicato nelle rispettive descrizioni. Nella Flora fossile della Gran Brettagna dei Signori Lindley e Hutton sono descritte e figurate due felci diverse solto il nome di Pecopteris dentata, l' una alla pagina 201 del Volume II. tav. 154 che è la vera Pecopteris dentata del Brongniart (Pecopteris Brongniartiana, Prest. Gyatheites dentatus, Görp.) e l'altra alla pag. 55 del Volume III. tav. 169, che è la testè descritta.

Essendovi così due Pecopteris col nome di $P$. dentata, era d'uopo, a scanso di equivoci, cangiare il nome ad una delle due, e fu ottimo consiglio quello del Presl di apporre alla presente specie il nome d'uno dei due illustri autori della Flora fossile della Gran Bretagna, chiamandola Pecopteris Huttoniana, nel che ho trovato indispensabile di seguirlo per togliere ogni confusione.

\section{Pecopteris insignis Lindl. Hutt.}

P. fronde bipinnata, rachide crassa, striata; pinnis remotis, alternis, patentibus, elongatis; pinnulis alternis oppositisque, adnatis, late-lanceolatis, falcatis, obtusis, integerrimis, approximatis, basi contiguis, sinu angusto discrelis; nervulis basi una vice furcatis e nervo medio dislincto excurrente angulo subacuto egredientibus, ramulis parallelis.

Pecopteris insignis. Lixnd. Hutr. Foss. Fl. Gr. Brit. II. pag. 69, tab. 106. - Strmab. Vers. Fl. Vorw. II. pag. 151, - Zigno. Enum. Filic. foss. form. Oolith. pag. 27.

Alethopteris insignis. Görp. Syst. Filic. foss. pag. 307. - Uxger. Synops. pag. 82 ; Gen. el Spec. Plant. foss. pag. 149. — Eichivald. Leth. Ross. Vol. II. pag. 15, tab. 2. fig. 6.

pteris insignis. Extrasas. Farrnkr. pag. 114. 
In arenaria inferiori ferruminosa formationis oolithicae ad Gristhorpe Bay et ad Cloughton prope Scarborough, Angliae; nec non in saxo calcareo ferruminoso, seriei Oxfordianae? inferioris, ad Kamenka prope Izoume in Russia.

E forse la specie più grande fra quelle che si conoscono della formazione oolitica. La fronda è bipinnata e munita di una rachide irregolarmente striata per lo lungo, il cui diametro misura cinque millimetri e dalla quale escono obbliquamente delle grandi pinne lunghe oltre venti centimetri e larghe un poco più di sei, appena picciuolate, patenti, distanti l'una dall'altra dai quattro ai cinque centimetri, e sorrette da rachidi, larghe due soli millimetri al punto d'inserzione, che si stringono gradatamente e diventano sottilissime verso l'estremità delle pinne. Le pinnule hanno il margine interissimo ed hanno una lunghezza di quattro centimetri, mentre alla base misurano poco più d'un centimetro. Sono generalmente alterne, però si osservano qua e là alcune paja opposte. La loro forma largamente lanceolato-falcata, partendo dalla base, si curva ed attenua verso l'estremità che è oltusa. Esse sono molto avvicinate, ma si mantengono divise fin quasi al punto d'inserzione sulla rachide, ove sono contigue e disgiunte soltanto da un seno angusto. Il nervo mediano è finissimo, ma bene distinto, e scorre fino all' apice della pinnula. I nervetti secondarj escono lateralmente da quello in senso obbliquo formando un angolo quasi acuto, e poco sopra la loro base si dividono in due ramoscelli semplici e paralleli che si prolungano fino al margine della pinnula.

Anche questa specie trovata a Gristhorpe Bay dai Signori Murray e Williamson è annoverata dal Signor Leckenby fra quelle che si trovano pure a Cloughton. Il chiarissimo Consigliere Eichwald, autore della Lethaea Rossica, ne descrive e figura un saggio trovato in Russia nell' argilla calcarifera e ferruginosa di Kamenka presso Izoume, che si ritiene appartenere alla parte più bassa degli strati Oxfordiani. Non saprei trovare alcuna analogia fra la presente specie e l'esemplare ad essa riferito dall'illustre Professore Göppert, e trovato a Wilmsdorf nell'argilla ferruginosa del terreno giurassico medio della Slesia. Vedi Göppert, Flor. d. m. Jura in Bericht. d. Nat. Sect. $d$. Schles. Gesellsch. 1845, pag. 144, tab. I. fig. 11.

Le pinnule, nel saggio figurato dal Göppert sono assai più corte e del doppio più larghe, ed i nervetti secondarj sono due e persino tre volte forcuti, coi ramoscelli divaricati. Questa figura s'avvicina piuttosto, per la nervatura e le proporzioni, a quella delle pinnule terminali della Pecopteris tenuis e della $P$. nebbensis del Brongniart, ma potrebbe pur anco spettare ad una specie nuova, certo non confondibile colla presente. 
7. Pecopteris Ilaiburnensis Lindl. Hutt.

P. fronde bipinnata, rachide striata; pinnis oppositis, sessilibus, patentibus, linearibus; pinnulis adnatis ovato-oblongis, obtusis, approximatis, basi subcontiguis; nervo medio tenui subflexuoso, apice furcato; nervulis tenuissimis, creberrimis, pluries furcatis, obliquis, arcuatis.

Pecopteris Haiburnensis. Lindu. et Hutrox. Foss. Flor. of Gr. Brit. III. pag. 97, Iab. 187. Prest. in Sterinb. Vers. Flor. Vorw. II. pag. 154. - Unger. Synop. pag. 100 ; Gen. et Spec. Plant. foss. pag. 179. - Zigno. Enum. Filic. foss. form. Oolith. pag. 27.

Cladophlebis Haiburnensis. Brorg. Tabl. Gen. Végél. foss. pag. 105.

Pteris Haiburnensis. Emrngsin. Farrnlir. pag. 114.

In formatione oolithica ad Haiburn Wyke prope Scarborough Angliae.

La fronda bipinnata ha una rachide primaria striata del diametro di appena tre millimetri da cui escono alla distanza di due centimetri e mezzo o tre, le pinne opposte sessili assai patenti e lineari, larghe circa tre millimetri ed ornate di pinnule ovato bislunghe assai ottuse ed avvicinate, inserite con tutta la base sulla rachide e quasi contigue, da prima un po' obblique, poi patenti, lunghe dai quindici ai venti millimetri e larghe dai sei agli otto. Il nervo primario che le percorre è molto sottile e dir potrebbesi capillare ed alquanto flessuoso, e sottilissimi sono gli assai numerosi nervicciuoli secondarii che obbliquamente ne escono ai lati, e biforcandosi più volte corrono piegandosi ad arco fino al margine della pinnula.

Questo esemplare trovato dal Prof. Phillips nei terreni oolitici di IIaiburn Wyke lungo la costa orientale della Contea di York in Inghilterra, è figurato nella Flora foss. della Gran Brettagna dei Signori Lindley e Hutton, ma, come avvertono gli autori, il disegnatore nell'ingrandimento sottoposto alla figura complessiva non rappresentò la base della pimnula come sta in natura, e la delineò affatto libera, mentre doveva segnarne l'inserzione sulla rachide con tutta la base.

Questa specie non trovasi illustrata nè nel Systema Filicum fossilium di Göppert, nè nella Histoire des Végétaux fossiles del Brongniart; quest' ultimo però l'inseri nell'elenco delle piante dell'oolite posto in calce al suo Tableau des Genres des Végétaux Fossiles, col nome di Gladophlebis Haiburnensis. 


\section{Pecopteris Odtonis Göpp.}

P. fronde bi vel tripinnata, rachide foliata, imbricato-squamosa; pinnis pinnulisque subpalentibus, pinnulis oblongo lanceolatis, obtusiusculis, inferioribus subpinnatifidis subremolis, superioribus subimbricalis, approximalis, integris, summis confluentibus; nervis secundariis e nervo medio subexcurrente, angulo acuto egredientibus, subobsoletis; soris subrotundis biserialibus.

Pecopteris Oltonis. Göpr. Flor. foss. d. m. Jura in Bericht. d. Arbeil. d. Schlessich. Gesellsch. etc. Breslau 1845-46, pag. 144, lab. 1. fig. 4-10. - Urg. Gen. et Spec. Plant. foss. pag. 174. (exclus. synon.) - Zigno. Enum. Filic. foss. form. Oolith. pag. 28.

In argilla ferruminosa formationis jurassicae mediae apud Matzdorf, Ludwigsdorf et Wilmsdorf in Silesia.

La fronda due e forse tre volte pinnata è munita di una rachide robusta del diametro di tre millimetri da cui si diramano obbliquamente le pinne quasi opposte ed un poco patenti. Negli intervalli frapposti alle pinne la rachide primaria è fornita di pinnule inserite per tutta la base, eguali a quelle che adornano le pinne. Le pinnule sono lanceolato-oblunghe un po' ottuse, le inferiori sono frastagliate e quasi pinnatifide e alquanto discoste fra loro, le superiori sono avvicinate quasi imbricate ed integre, e quelle vicino all'apice sono confluenti, ma tulte in generale sono contigue alla base ed inserite per intero sulla rachide. La larghezza delle pinne oltrepassa i due centimetri, quella delle pinnule varia dai tre ai quattro millimetri sopra una lunghezza d'un centimetro ad uno e mezzo. Oggni pinnula è percorsa da un sottil nervo mediano fiancheggiato da esilissimi nervi uscenti ad angolo acuto dai suoi lati e poco visibili. La fruttificazione è composta di sori quasi rotondi disposti in serie ai lati del nervo mediano.

Questa specie fu trovata nell' argilla ferruginosa del terreno giurassico medio a Matzdorf, Ludwigsdorf e Wilmsdorf della Slesia, e non deve, a parer mio, confondersi coll'Alethopteris Ottonis Göpr. Syst. Fil. foss. pag. 303, tab. 37, fig. 3. 4. di cui è sinonimo la Pecopteris Ottonis Press. in Sternis. Vers. Fl. Vorw. II. pag. 161, e che fu rinvenuta nella formazione del carbon fossile a Wielun nella Polonia. E forza però confessare che tanto la descrizione che le figure sono un poco confuse, e che questa specie meriterebbe un nuovo esame per parte dell'illustre suo scopritore. 


\section{Pecopteris Phillipsii Brong.}

P. fronde bipinnata, rachide crassa, striata; pinnis alternis, sessilibus, patentibus, lineari-lanceolatis, elongatis, obtusiusculis, profunde pinnatifidis; laciniis vel pinnulis integris, patentibus, alternis, linearibus oblongis, acutiusculis, ima basi connatis rachimque marginantibus, sinu angusto obtuso discretis, marginibus convolutis; nervo medio valido excurrente; nervis secundariis remotis, obliquis, una tantum vice medio furcatis, ramulis divaricatis.

Pecopteris Phillipsii. Brovas. Prodrom. pag. 57; Hist. Végét. foss. I. pag. 304, tab. 109, fig. 1.

- Stensa. Vers. Fl. Vorw. II. pag. 150. - Zıgno. Enum. Filic. fuss, form. Oolith. pag. 28.

Alethopteris Phillipsii. Görr. Syst. Filic. foss. pag. 304. - Unger. Gen. et Spec. Plant. foss. pag. 148.

In formatione oolithica ad Cayton prope Scarborough Angliae, invenit Gl. Williamson.

La fronda piuttosto larga e bipinnata è munita di una rachide grossa, sporgente, striata per lo lungo ed avente un diametro di oltre quattro millimetri. Le pinne lunghe sette centimetri e larghe circa dieciotto millinıtri sono alterne, patenti e fornite esse pure di una rachide robusta e striata. Hanno forma lineare lanceolata, allungata, un poco ottusa all' apice, e sono profondamente pinnatifide. Le pinnule o lacinie, lunghe nove millimetri e larghe uno e mezzo, sono pure alterne e leggermente discoste fra di loro verso le estremità, ma si congiungono presso la base e con essa formano ala alla rachide, separate soltanto da un seno angusto ed ottuso un po' prima del punto d'inserzione ove sono contigue. Sono lineari bislunghe, interissime, coll'estremità appuntita e coi margini leggermente ricurvi verso la pagina inferiore. Un nervo mediano appariscente le percorre ritto fino all' apice, e da questo escono obbliquamente i nervelti secondarii che nel mezzo si fanno una sol volta forculi, dividendosi in due soli ramoscelli molto divaricati.

Questa specie, che non è amnoverata nella Fossil Flora of Great Britain dei Signori Lindley e IIutton, fu trovata nella formazione oolitica d'Inghilterra a Caython presso Scarborough dal chiarissimo Signor Williamson. 


\section{Hecopteris ligata Phillips.}

P. fronde bipinnata, rachide aequali medio sulcata; pinnis oppositis, patentibus, elongatis; pinnulis alternis, integerrimis, lanceolato-acuminalis, falcatis, base sensim dilatata rachi adnatis, approximatis, sed usque ad basim discretis; nervis secundariis, e nervo medio excurrente, angulo acuto egredientibus, oppositis, unifurcatis, arcuatis.

Pecopteris ligata. Pmulirs. Geol. of Yorkish. I. pag. 153, tab. 8. fig. 14. - Monns. Cat. Brit. foss. pag. 16. - Zigro. Enum. Filic. foss. form. Oolith. pag. 28.

Pecopteris Whitbiensis. Linde. Ilutr. Foss. Fl. Gr. Brit. II. pag. 145, tab. 134 . (exclus, synen. Brovg.)

Pecopleris Whitbiensis var. (a) Lindleyana, laciniis acuminalis, venis unifurcatis. Prest. in Sterna. Vers. Fl. Vorw. II. pag. 150.

Alethopteris Whitbiensis. Görr. Syst. Filic. foss. pag. 304. - Urger. Gen. et Spec. Plant. foss. pag. 148. - Eichwald. Leth. Ross. Vol. II. pag. 16.

In argilla ferruminosa formationis oolithicae ad Cloughton prope Scarborough Angliae, nec non in formatione jurassica inferiori in Daghestan al Caucasum juxta $\mathrm{Cl}$. Eichwald.

La fronda piuttosto grande e bipinnata è percorsa da una rachide segnata nel mezzo da lieve solco longitudinale. La rachide ha un diametro di due millimetri, e si mantiene eguale per tutto il tratto visibile sull' esemplare figurato nella Fossil Flora of Great Britain, e che raggiunge una lunghezza di quattordici centimetri. Le pinne sono opposte e patenti, uscendo quasi ad angolo retto dalla rachide primaria e distano l'una dall'altra, al punto d'inserzione, circa due centimetri. Verso la base sono larghe due centimetri, ma poscia procedendo verso l'apice si fanno gradatamente piu anguste in guisa, che ad una distanza di sei centimetri dalla base, la loro larghezza si riduce a dodici millimetri. Mancano nell'esemplare tutte le estremità delle pinne, ma dalle esposte misure si può arguire che la loro lunghezza non fosse minore degli otto centimetri. Le pinnule sono alterne, interissime, di forma lanceolata falcata molto acuminata, leggermente incurve verso l'apice e lunghe circa quindici millimetri. Gradatamente si allargano procedendo verso la base ove misurano dai quattro ai cinque millimetri, e s' inseriscono per intero ed un poco obbliquamente sulla rachide, rimanendo fra loro disgiunte, quantunque avvicinate in guisa da toccarsi al punto d'inserzione. Il nervo me- 
diano scorre ben marcato fino all' apice della pinnula, ed escono da questo ad angolo acuto i nervetti secondarii, che poco sopra la loro origine si fanno una sol volta forcuti, e si volgono con lieve curva verso i margini laterali della pinnula.

Questa specie fu trovata nelle arenarie e nei noduli di argilla ferruginosa della formazione oolitica a Cloughton presso Scarborough nell'Inghilterra dal Prof. Phillips e dal Signor Williamson. Il chiarissimo Consigliere. Eicliwald la annovera nella sua Lethaea Rossici, come proveniente dal terreno giurassico inferiore del Daghestan nel Caucaso.

La figura che di questa specie ci porge il Prof. Phillips (Geol. Yorksh. tav. 8. fig. 14), sebbene lasci molto a desiderare, corrisponde a quella assai migliore che i Signori Lindley e Hutton riferirono alla Pecopteris Witbiensis del Brongniart (Foss. Fl. Gr. Brit. Vol. II. tav. 134). Tutte due però sono affatto diverse dalle figure della Pecopteris Witbiensis delineate nella Histoire des Végétaux fossiles, alla tav. 109, fig. 2. 3. 4. In quest' ultime la rachide è segnata da più solchi paralleli, le pinne, sebbene opposte nella parte inferiore della fronda, sono alterne nella parte superiore, ed hanno una direzione obbliqua, invece d'essere patenti come nella presente specie. Le pinnule sono assai meno acuminate, ed invece d'essere separate fra loro, si congiungono alla base formando ala alle rachidi secondarie. I nervetti secondarii sono ora opposti, ora alterni e quasi tutti due volte forcuti coi ramoscelli delle inforcature piuttosto divaricati, mentre nella $\mathrm{Pe}$ copteris ligata PHiL. sono costantemente opposti, una sol volta forcuti, coi ramoscelli avvicinati, arcuati e rivolti all'infuori.

Tanto il Prof. Phillips, nell'opera testè nominata, quanto il Signor Morris nel suo Catalogue of British fossils pag. 16, citano a proposito della Pecopteris ligata Pumc., la figura della Pecopteris Whitbiensis data dai Signori Lindley e Hutton, ma non riferiscono ad essa la $P$. Whitbiensis del Brongniart, che anzi dal Morris viene annoverata separatamente (loc. cit. pag. 17) quale specie diversa. Nell'opera dello Sternberg il Presl calcola la $P$. Whitbiensis figurata dai Signori Lindley e Ilutton quale una varietà, e la distingue colla frase $P$. Whitbiensis var. a. Lindleyana, laciniis acuminatis, venis umifurcutis. Göppert ponendo questa forma nel genere Alethopteris, cita egli pure la figura della Fossil Flora of Great Britain, e fa della Pecopteris Whitbiensis del Brongniart la varietà $\beta$. Brongniartii, pimmulis majoribus latioribus, nervis secundariis dichotomis, ramulis furcatis.

Nella frase specifica non è poi accennato il carattere delle pinne opposte e quello assai più importante dei nervetti secondarii una sol volta forcuti, mentre invece li descrive per dicotomi, la quale espressione, come è noto, significa ripetutamente e più volte forcuti. L'Unger riporta inalterata la frase specifica che accompagna l'Alethopteris 


\section{$-142$}

Whitbiensis di Göppert, e riguardo alla specie lo segue nella citazione della figura della Fossil Flora of Great Britain, ma sotto alla var. B. Brongniartii di Göppert, colloca quali sinonimi la $P$. Whitbiensis Brong., la P. tenuis Brong. e le varietà Lindleyana e Brongmiartii di Presl.

Dal fin qui detto mi sembra chiaramente emergere, che la Pecopteris ligata di Phillips e la Pecopteris Whitbiensis figurata dai Signori Lindley e Ifutton sieno una sola e medesima specie, affatto distinta dalla Pecopteris Whitbiensis descritta e figurata dal Brongniart, ed è perciò che allo scopo di evitare ogni confusione io qui la descrissi conservandole il nome che le fu imposto da Phillips, e dandomi ogni cura per rettificarne la sinonimia.

\section{Hecopteris Whidbiensis Brong.}

P. fronde ampla, bipinnata; rachide crassa pluries sulcata, sulcis parallelis remotiusculis; pinnis obliquis, linearibus, elongatis, apice attenuatis, inferioribus oppositis, superioribus alternis; pinnulis contiguis, aequalibus, lanceolatis, acutis, inflexis, subarcuatis, basi, inferius contracta superne protracla tantum conjunctis, sinu subrotundo, angustissimo, discrelis; nervo medio crassiusculo, excurrente, apice furcato; nervis sccundariis obliquis, oppositis alternisve, dichotomis, ramulis divaricatis subinflexis.

Pecopteris Whitbiensis. Beose. Prodrom. pag. 57; Hist. Végét. foss. 1. pag. 321, tab. 109 , fig. 2. 3. 4. - Puncirs. Geol. of Yorksh. I. pag. 153. - Monis. Cal. Bril. foss. pag. 17. Zigxo. Enum. Filic. foss. form. Oolith. pag. 28.

Pecopleris Whitbiensis var. $\gamma$. Brongniartii, laciniis acutis oblusisque vix faleatis, venis bitrifurcatis. Presl in Sternib. Vers. Fl. Vorw. II. pag. 450.

Pecopteris Whitbiensis var. $\beta$. Brongniartii, pinnulis majoribus latioribus, nervis secundariis dicholomis, ramulis fureatis. Görp. Syst. Filic. foss. pag. 304 . - Urger. Gen. el Spec. Plant. foss. pag. 148. (excl. synon. P. Whitbiensis var. a. Lindleyanae STEnxв.)

Pecopteris hastata. Pnulurs. Geol. of Yorksh. I. pag. 119, 153, lab. 8. fig. 17.

Pecopteris tenuis. Brong. Hist. Végét. foss. I. pag. 322, tab. 110, fig. 4.

In formatione oolithica ad Whitby et Scarborough in Gomitatu York, Angliae.

La ragguardevole grossezza della rachide di uno fra i varii esemplari figurati dal Brongniart, dà a divedere come fosse assai grande la fronda di questa specie. Però, come giustamente osserva lo stesso Brongniart, da nessuno dei saggi raccolti si può 


\section{$-145-$}

trarre argomento per ammettere che fosse tripinnata, e quindi dobbiamo ritenere che ad onta della sua ampiezza fosse soltanto bipinnata, come avviene eziandio in parecchie Felci della Flora attuale.

La rachide primaria ha nella sua parte inferiore un diametro di dodici millimetri, ed è segnata per lo lungo nella sua faccia anteriore da quattro solchi paralleli, discosti l'uno dall'altro due millimetri. Nella parte superiore della fronda il diametro della rachide decresce fino ai tre millimetri, ed allora è segnata nel mezzo da un solo solco longitudinale. Le pinne sono obblique, lineari, larghe al basso dai quindici ai ventisei millimetri e molto allungate, poichè se ne veggono delle porzioni lunghe nove centimetri, la cui larghezza indica mancare ancora un bel tratto per giungere all' estremità, verso la quale, nelle pinne più piccole e meno incomplele, si scorge come vadano lentamente attenuandosi. Le pinne inferiori sono opposte, le superiori invece sono alterne, e le rachidi secondarie onde sono percorse hanno una depressione nel mezzo e leggermente s'ingrossano a guisa di picciuolo nel punto d'inserzione sulla rachide principale. Le pinnule, negli esemplari più grandi, misurano tredici millimetri in lunghezza sopra sei di larghezza. Esse sono contigue, eguali, lanceolate acute, e descrivono una lieve curva coll' apice diretto verso l'estremità della pinna. La base è nella parte sua inferiore leggermente contratta, mentre si protende alcun poco col lembo superiore, e per esso soltanto si congiungono fra di loro le pinnule, formando ivi un seno rotondato assai angusto e rimanendo divise in tutto il resto della loro lunghezza. Per questa disposizione della base delle pinnule le rachidi secondarie riescono brevemente alate. Il nervo primario è inferiormente piuttosto rilevato, poi scorre, assottigliandosi e facendosi un poco flessuoso, fino all'apice della pinnula, ove termina forcuto. I nervetti secondarii escono assai obbliquamente da quello talvolta opposti, ma più spesso alterni, biforcandosi due o tre volte, e coi ramoscelli delle ultime inforcature divaricati e leggermente arcuati l'un verso l'altro.

Questa specie proviene dalle argille schistose della formazione oolitica delle vicinanze di Wilhby e di Scarborough in Inghilterra.

Si citano saggi della presente specie provenienti anche dai terreni liassici della Stiria, della Baviera e del Banato, per cui riuscirebbe promiscua a due formazioni, e come tale viene pure ritenuta da alcuni autori. Ma la identità di questi saggi colla Pecopteris Whilbiensis dell'Inghilterra non mi sembra per anco pienamente provata. E sarebbe, a dir vero, di somma importanza che venissero scrupolosamente confrontati con quelli dell'oolite di Whitby per chiarire se realmente appartengano alla stessa specie. Io non mi farò qui a negare la possibilita che qualche fossile possa trovarsi promiscuo fra 


\section{$-144-$}

due terreni, specialmente se prossimi nell'ordine cronologico delle formazioni, tuttavia devo dichiarare che ben di spesso ho veduto come il diligente esame dei caratteri differenziali abbia sovente fatto sparire queste asserite identita di specie, comuni a due formazioni diverse.

\section{Peopteris exilis Phillips.}

P. fronde tripinnata, rachide crassiuscula, sulco medio longitudinaliter instructa; pinnis elongatis laxis, late lanceolatis, rachidibus secundariis gracilibus, sulcato-carinatis; pinnulis alternis, oppositisve, patulis, lincaribus, acuminatis, apice obtusiusculis, profunde pinnatifidis; laciniis ovato-linearibus, integris, obtusissimis, patentibus, alternis, sinu subrolundo angusto discretis, remotiusculis; nervis secundariis, e nervo medio excurrente, angulo acuto egredientibus, remoliusculis, arcuatis, furcatis; sporangiis solitariis, magnis, ovato-globosis, apice umbilicatis, annulo stellatim multiradiato instructis, juxta nervum medium seriatim dispositis.

Pecopteris exilis. Geol. of Yorksh. I. pag. 149, lab. 8. fig. 16. - Momus. Cal. Brit. foss. pag. 16. - Bunbury. On foss. Pl. from the Yorksh. Coast in Quart. Yourn. Geol. Soc. Lond. Vol. VII. pagg. 188, tab. 13. fig. 5. a, 6. - Lecrenвy. On the Sandst. and Sh. of Scarb. etc. in Quart. Yourn. Geol. Soc. Lond. Vol. XX, pag. 76

Neuropteris obtusifolia. Munnar. MSS.

Pecopteris oblusifolia. Lindu. Hotт. Fuss. Fl. Gr. Brit. III. pag. 17, lab. 158, fig. 1. a, b, tab. 157, fig. 1. c. - Stenra. Vers. Fl. Vorw. II. pag. 153.

Cyatheites obtusifolius. Görp. Syst. Filic. foss. pag. 328. - Urgen. Gen. et Spec. Plant. foss. pag. 159. - Zigro. Enum. Filic. foss. form. Oolith. pag. 29.

In argilla inferiori formationis oolithicae ad Gristhorpe prope Scarborough Angliae.

Dagli esemplari che mi furono favoriti dal Signor Leckenby riesce palese la forma tripinnata della fronda, che è sorretta da una rachide robusta del diametro di quattro millimetri, e percorsa nel mezzo da un solco longitudinale. Le pinne, alquanto sparpagliate, e distanti l'una dall'altra dai tre ai quattro centimetri, hanno una rachide piuttosto gracile, solcata nella faccia anteriore e carenata nel dorso, che ha un diametro di circa un millimetro; e si palesano di forma largamente lanceolata. Dai frammenti che si osservano sui saggi si può calcolare che raggiungessero una lunghezza non minore 


\section{$-145-$}

di quattordici centimetri. La larghezza poi si palesa di circa sei centimetri. Le pinnule ora alterne, ora opposte sono patenti, discoste l'una dall'altra al punto d'inserzione cinque millimetri all'incirca, lunghe fino a tre centimetri e larghe dai quattro ai sei millimetri, di forma lineare acuminata, coll'apice un poco ottuso, e profondamente pinnatifide. Le lacinie sono ovato-lineari interissime, assai ottuse o, per meglio dire, rotondate all'apice, alterne, patenti, disgiunte fra loro e separate verso la base da un seno rotondato ed angusto, sotto il quale si congiungono e formano ala alla rachide. Esse misurano dai tre ai quattro millimetri di lunghezza sopra una larghezza di un millimetro e mezzo o due. Ogni lacinia è percorsa fino all' estremità da un nervo mediano assai marcato, dai cui lati escono obbliquamente e quasi opposti i nervetti secondarii una sol volta forcuti e lievemente arcuati. La fruttificazione si manifesta composta di sporangi solitari di ragguardevole grandezza, ordinati in serie regolare lungo cadaun lato del nervo principale delle lacinie. Essi hanno una forma ovato-sferica, e sono lievemente ombilicati nel vertice, ove si palesano forniti di un annello composto da una serie di cellule raggianti dal punto centrale ombilicato.

Gli esemplari di questa specie si trovano nelle argille inferiori dei terreni oolitici di Gristhorpe presso Scarborough nell'Inghilterra.

La fruttificazione si scorge segnata sulle figure pubblicate dai Signori Phillips, Lindley e Hutton, che la credettero composta di sori rotondi posti lungo il nervo mediano delle lacinie, e come tale la descrissero. Basato su queste indicazioni il Professore Göppert collocava la presente specie, unitamente a quella che segue, nel suo genere Cyalheites, ma le accurate indagini fatte di poi da Sir C. Bunbury, svelarono delle analogie con altri generi ben diversi. Questo acutissimo osservatore giunse a riconoscere, che la fruttificazione della Pecopteris exilis non si componeva di sori racchiudenti un aggregato di sporangi, ma bensi constava di sporangi isolati, di considerevole grandezza, coll'apice fornito di un annello composto di cellule disposte a raggio intorno ad un punto centrale più depresso. E questa interessante scoperta, mentre da uin lato ci vieta di più annoverare queste forme nel genere Gyatheites, che il Göppert stabilisce provveduto di sori muniti dell'indusio, svela dall'altro canto un modo di fruttificazione, che piuttosto s'avvicina a quello dei generi Aneimia e Mohria di Swartz, ed Anemidictyon di Smith, della Flora attualmente vivente, ed al genere Senftenbergia di Corda della Flora fossile dell' epoca carbonifera. Tuttavia il chiarissimo Sir C. Bunbury non ha creduto opportuno di creare un nuovo genere in cui accogliere questa nuova forma, ed ha preferito restituirla al genere Pecopteris nel quale era stata collocata da Phillips. Nel che divido perfettamente la sua opinione, e perciò reputo miglior 


\section{$-146$}

consiglio il conservare tanto questa specie, quanto la seguente nel genere cui maggiormente s'avvicinano tanto per la forma delle lacinie, che per il modo di distribuzione dei nervi.

\section{Pecopteris acutifolia Lindl. Hutt.}

P. fronde tripinnata, rachide unisulcata; pinnis elongatis, late lanceolatis, patentibus, remotis, rachidibus secundariis sulcato-carenalis subflexuosis; pinnulis linearibus acuminatis profunde pinnatifidis, alternis, patulis, extremis oppositis obliquis; laciniis linearibus angustis, integris, acutis, patentibus, alternis, remotis, ad rachidem sinu oblique lanceolato subacuto discretis, decurrentibus; nervis secundariis tenuissimis, e nervo medio tenui excurrente, angulo aculo egredientibus, remotiusculis, arcuatis, furcatis.

Pecopteris acutifolia. Linde. Hutr. Foss. Fl. Gr. Brit. III. pag. 13, tab. 157, fig. 2. 2, b; lab. 158, fig. 2. 2, a. - Stennb. Vers. Fl. Vorw. II. pag. 153. - Lechenny. On Sandst. and Sh. of the Ool. of Scarb. etc. in Quart. Journ. Geol. Soc. Lond. Vol. XX. pag. 76.

Neuropteris aculifolia. Munnay. MSS.

Cyatheites acutifolius. Görp. Syst. Filic. foss. pag. 328. - Unger. Gen. et Spec. Plant. foss. pag. 160. - Zisno. Enum. Filic. foss. form. Oolith. pag. 29.

Invenitur eodem in loco cum praecedenti.

La fronda anche di questa specie apparisce tripinnata, per la forma profondamente pinnatifida delle pinnule, ed è sorretta da una rachide un po' più angusta, segnata nel mezzo da un solco longitudinale. Le pinne sono pure ampiamente lanceolate, molto patenti e remote, e le rachidi secondarie, nello stesso modo solcate per lo lungo sul dinanzi e carenate sul dorso, sono leggermente flessuose. Le pinne sembrano aver raggiunto la medesima lunghezza di quelle della specie precedente, ed averle superate di un centimetro o poco più nella larghezza. Le pinnule sono lineari, acuminate, profondamente pinnatifide, lunghe quattro centimetri e larghe dai cinque ai sei millimetri, alterne e patenti. Però quelle collocate verso le estremità delle pinne sono invece opposte ed obblique. Le lacinie sono lineari, anguste, coll'apice acuto, lunghe dai quattro ai cinque millimetri e larghe poco oltre un millimetro. Sono patenti, alterne, alquanto l'una dall'altra discoste, e separate fino alla rachide sulla quale decorrono divise da un seno obbliquamente lanceolato e quasi acuto. Il nervo mediano è soltile e scorre fino all' apice della lacinia. I nervetti secondarii sottilissimi, sorgono dal nervo mediano ob- 


\section{$-147$}

bliquamente ed un poco discosti l'uno dall'altro, facendosi un po' arcuati ed una sol volta forcuti come nella specie antecedente.

Gli esemplari di questa specie si trovano frequentemente, con quelli della precedente, negli stessi luoghi.

Le differenze che distinguono questa specie dall'antecedente sono, a dir vero, poco rilevanti e tali, da indurre nel sospetto che possa essere una semplice varietà. Però, confrontando i saggi della Pecopteris exilis con quelli della Pecopteris acutifolia, si scorge come le pinnule collocate verso l'estremità delle pinne, sieno in quest' ultima più obblique e più costantemente opposte. Le lacinie poi sono tutte più anguste e coll'apice acuto, e tutti i nervi si palesano relativamente più šottili.

\section{Pecopteris ctenis Pomel.}

P. fronde...., pinnulis lineari-oblongis, subfalcatis, obtusis, usque ad rachidem discrelis, sinu rotundato angusto divisis, basi dilatata decurrenti conjunclis; nervo medio excurrente, nervulis obliteratis.

Pecopteris ctenis. Pones. Matériaux pour servir à la Flore Jurass. de la France, in Amil. Bericht. d. Gesellsch. Deutsch. Nalurf. U. Erzte. Aachen 1847-48, pag. 339, n. 3. - Zıмхo. Enum. Filic. foss. form. Oolith. pag. 29.

In formatione coralliana seriei oolithicae mediae ad S.' Michel in Gallia.

1) Pomel non dà alcuna indicazione circa alla forma della fronda, ed accenna soltanto che le pinnule sono lineari oblunghe, un poco falcate, ottuse, divise fino alla rachide, ove sono separate da un seno rotondato ed assai angusto, e congiunte per la base, che è dilatata e decorrente. Un solo nervo mediano le percorre, dal quale, suppone il Pomel, uscissero i nervetti secondarii, di cui però confessa non rimanere traccia alcuna.

Questo saggio fu trovato nel piano coralliano della serie oolitica media presso S.' Michel in Francia.

Io conservo con qualche esitanza questa forma nel genere Pecopteris in cui fu collocata dal Pomel. Tutti i caratteri di cui fa menzione quell'autore, indurrebbero piut- 


\section{$-148$}

tosto a toglierla dalle Felci e porla nel genere Gycadites; ma senza un accurato esame degli esemplari sarebbe avventatezza il farlo. Perciò conviene attenersi alle indicazioni, per dir il vero, assai superficiali, che ci vengono somministrate dallo scopritore di questa curiosa forma vegetale.

\section{Pecopteris Ittieri Pomel.}

P. fronde crassa; pinnulis tota base rachi adnatis, subcontiguis, usque ad rachidem discretis, oblongis, apice obtusis, rotundatis, terminalibus majoribus, lanceolatis, obtusis, basi inaequali plus minusve auriculatis; nervo medio crasso excurrente; nervulis secundariis obliteratis.

Pecopteris Ittieri. Pones. Matériaux p. la Fl. Jurass. de la France, in Amtl. Bericht. d. Gesellsch. Naturf. u. Erzle. Aachen 1847-48, pag. 339, n. 4.-Zigno. Enum. Filic. foss. form. Oolith. pag. 29

In eadem formatione ad Seyssel in Gallia.

Dice il Pomel, che questa specie, per la sua forma generale, rammenta un poco l'aspetto delle Neurotteridi. Sogggiunge però tosto, che le pinnule aderiscono alla rachide con tutta la base, e che un nervo mediano assai grosso le percorre fino all' apice. Della fronda non indica che il rilevante spessore, ed ommette di parlare della forma, la quale deve essere stata almeno pinnata, poichè subito dopo ci favella di pinnule, e le descrive di forma oblunga, ottuse e rotondate all'apice, quasi contigue, ma divise fino alla rachide, aggiungendo che le pinnule terminali sono piu grandi, lanceolate, ottuse, colla base ineguale, e più o meno auricolate. In quanto alle affinità ch' egli accenna sussistere fra questa forma ed alcune specie dei generi Neuropteris, Geterach e Maranta, non vale la pena di farne menzione.

Anche questa specie proviene dagli strati dell'oolite media, e fu rinvenuta a Seyssel nel Bugey in Francia. 


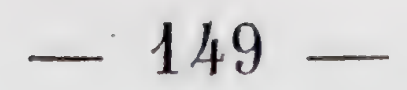

Species nondum descripta.

\section{Pecopteris Moretiana Brong.}

In formatione coralliana seriei oolithicae mediae, invenit Gl. Moret ad Chatillon sur Seine in Gallia. Brong. Tabl. Gen. Végét. foss. pag. 105, 106.

Questa forma non fu ancora descritta. Essa si trova semplicemente annoverata dal Brongniart nell' elenco delle piante proprie dell' epoca oolitica, posto in fine del suo Tableau des Genres des Végétaux fossiles, e fu da lui dedicata al Colonnello Noret che la rinvenne in Francia presso Chatillon sur Seine in un terreno spettante al piano corálliano dell' Oolite media.

\section{Gen. XX. Achostichutes Göpp.}

Frons bipinnata. Pinnulae rachi adnatae. Nervus medius tenuis. Nervi secundarii, e nervo medio oblique egredientes, arcuati dichotomo-furcati. Fructificatio, capsulis (sporangiis) confertis, partim vel totam paginam inferiorem pinnularum occupans.

Acrostichites. Göpr. Syst. Filic. foss. pag. 174, 284 (pro parte). - Unger. Synops. pag. 76 ; Gen. et Spec. Plant. foss. pag. 140 (pro parte).

\section{Merostichites Villiamsonis Göpp.}

A. fronde bipinnata, rachide crassa striato-sulcata; pinnis linearibus, elongatis, oppositis, subpatentibus; pinnulis approximatis vel remotiusculis, suboppositis alternisve, patentissimis, elliptico-arcuatis, integerrimis, apice rotundatis, tota base dilatata rachi adnatis, pagina inferiori, capsulis (sporangiis) arcte confertis, omnino vel partim, obtecta; nervo medio tenui, subflexuoso; nervis secundariis obliquis, arcualis, dichotomo-furcatis.

Acrostichites Williamsonis. Göpr. Syst. Filic, foss. pağ. 285. - Ungen. Gen. el Spec. Plant. foss. pag. 141. - Bunnurx. Foss. Plants from. Scarb. in Quart. Journ. Geol. Soc. Lond. 1851. 
Vol. ViI. pag. 187. - Brosx. Lethaea Geogn. II. pag. 54, tab. 44. fig. 2. - Eicuwald. Leth. Ross. II. pag. 17. tab. 2. fig. 3. - Zısvo. Enum. Filic. foss. form. Oolith. pag. 29.

Pecopteris Williamsonis. Brosg. Prodrom. pag. 53; IIist. Végét. Toss. I. pag. 334, tab. 110, fig. 1. 2. - Linde. el Hutt, Foss. Fl. Gr. Brit. II. Lab. 126. - Stennis. Vers. Fl. Vorw. II. pag. 151 .

Pecopteris curtata. Pniluris. Geol. of Yorksh. 1. pag. 119, 125, tab. 8. fig 12. el tab. 10. fig. 7 .

Cladophlebis Williamsonis. Bnoxc. Tabl. Gen. Végét. foss. pag. 105.

Acrostichum Williamsonis. Eтtrasir. Farrnkr. pag. 21.

In stratis arenaceo-argillosis formationis oolithicae ad Gristhorpe et ad Cloughton prope Scarborough, Angliae; nec non in schisto argilloso micaceo nigrescente formationis jurassicae ad Räscht et Kasbine, aliisque in locis, prope Ghilan et Mazanderan in Persia, juxta Gl. Eq. de Eichwald.

Un esemplare di questa bella specie, favoritomi dal chiarissimo Signor Leckenby di Scarborough, misura trentadue centimetri in lunghezza e dieciotto di larghezza, e tuttavia è mancante dell'estremità superiore e della parte inferiore della fronda, dal che possiamo inferire che essa giungesse ad una dimensione piuttosto ragguardevole; tanto più che il diametro della rachide primaria, il quale al basso misura poco più di otto millimetri, verso l'estremità del saggio si riduce soltanto a sei, cosi diminuendo gradatamente di soli due millimetri, sopra un tratto di ventotto centimetri all'incirca di lunghezza. La fronda è bipinnata colla rachide principale lievemente striata, percorsa da qualche solco longiludinale e depressa nel nezzo. Le pinne sono opposte e quasi patenti, lineari, larghe dieci millimetri, lunghe dai dieci ai dodici centimetri, discoste l'una dall'altra, al punto d'inserzione, circa dodici millimetri. La rachide secondaria che le sorregge, sebbene non superi nel diametro il millimetro e mezzo, apparisce però alquanto robusta e pure segnata nel niezzo da una depressione longitudinale poco profonda. Le pinnule sono interissime, quasi opposte, assai patenti e molto avvicinate tra loro; però nelle parti superiori della fronda sono all'invece alterne ed un poco discoste l'una dall'altra. Hanno una forma che il Göppert definisce per ellittico-arcuata, ma clie potrebbe anche dirsi obovato-falcata od incurva, coll' apice ottuso e rotondato e colla base dilatata ed inserita per intero sulla rachide secondaria. La loro larghezza è di tre millimetri e non oltrepassano i cinque in lunghezza. Il nervo mediano è sottile, ma ben marcato ed un poco flessuoso, ed i nervetti secondarii uscendo obbliquamente da quello, si fanno poscia leggermente arcuati, e due volte forcuti. La fruttificazione non porge alcun indizio di sori, e consta invece di un aggregato di capsule 


\section{$-151$}

(sporangi) fittamente addensate, che ricoprono per intero, od in parte, la pagina inferiore delle pinnule.

Questa specie si rinviene nelle stratificazioni arenaceo-argillose della serie oolitica, tanto a Cloughton che a Gristhorpe presso Scarborough nell' Inghilterra, e trovasi pur anco, secondo il chiarissimo autore della Lethaea Rossica, in uno schisto nero argilloso micaceo, spettante alla formazione giurese, a Räscht ed a Kasbine, ed in altri luoghi del Ghilan e del Mazanderan in Persia.

La fruttificazione, già da gran tempo riconosciuta dai Signori Dunn, Lindley e IIutton, e da questi ultimi figurata e descritta, fu poscia accuratamente esaminata da Sir C. Bunbury, sopra un esemplare esistente nella collezione del Dott. Murray. E potè il Bunbury convincersi che i corpicciuoli rotondi e stipati, i quali cosi di sovente ricoprono la pagina inferiore delle pinnule, non sono squame od altre appendici della cuticola, come sospettava il Presl, ma bensi capsule (sporangi), che nel citato esemplare del Sig. Nurray, si manifestano cosi bene conservate e cosi poco compresse, da poter facilmente, col semplice ajuto di una lente, riconoscerne la forma e struttura affatto simile a quella degli sporangi delle Felci viventi. Osserva inoltre, assai giustamente, Sir C. Bunbury, che la distribuzione di questi organi su tutta la pagina inferiore delle pinnule, o su parte di essa, presenta una decisa analogia col modo in cui è distribuita la fruttificazione sulla fronda di varie Acrosticacee della Flora attuale, e comunque per altri caratteri la presente forma se ne allontani, consiglia però a conservarla nel genere Acrostichites, come quello che accenna alla sua affinita col genere Acrostichum preso in largo senso ed avuto riguardo soltanto alla fruttificazione senza tener conto della nervalura. Ed io tanto più volentieri mi acconcio al parere di Sir C. Bunbury, in quanto che, come si è veduto, ho escluso dal genere Acrostichites, tutte le forme a nervi reticolati che Göppert ed Unger vi avevano riunite, ma che lo stesso Göppert separava di poi da questo genere e collocava nel genere Sagenopteris di Presl.

\section{Gen. XXN. Crcamopterens Zigno.}

Frons pinnatifida, vel pinnata aut bipinnata, rachide alata, striata, basi incrassata. Laciniae vel pinnulae integrae, coriaceae, marginatae, secus rachidem alatam decurrentes. Nervi primarii crassissimi, lati, striati, excurrentes, apice furcati. Nervi 
secundarii crassi e nervo medio oblique egredientes, arcuali, unifurcati, interdum simplices. Sori lineares, arcuati, juxta nervulos dispositi, immersi, sub cuticula nascentes. Cuticula, sporangiorum maturitate fissa, fissura arcuata, lineari-oblonga, marginibus labiiformibus inaequaliter incrassatis.

Filices elasticae, facie Polypodiorum, frondem elasticam, coriaceam pectinatam gerentes.

Zigro. Cicad. foss. dell'Oolite. in Rio. period. 1. R. Accad. Pad. 1853 ; Entdeck. foss. P $l$. d. Jura d. Venet. Alp. in Neu. Jahr. Min. Geogn. Geol. und Petrefactenk. von Leonh. u. Bronn, 1854, pag. 34; Nuovo Gen. di Fel. foss. in Act. I. R. Istit. Ven. Vol. VI. Ser. III. 1861; Enum. Filic. foss. form. Oolith. pag. 29.

Fino dalla prima volta che in'imbattei in alcuna delle varie forme, che poscia riunii in questo nuovo gruppo generico, entrai nella persuasione che spettassero alla classe delle Felci; quantunque, nei primi esemplari raccolti, niuna traccia si potesse scorgere della fruttificazione, nè visibili fossero i nervetti secondarii (1). Ed è perciò, che quando nel 1853 recai meco in Vienna questi saggi, e li sottoposi al gitudizio del chiarissimo Professore Unger, egli mi esternava l'opinione che dovessero piuttosto appartenere ad un nuovo genere di Cicadeacea. L'autorità di un tal parere $m$ 'indusse quindi ad annoverarle fra le piante di questa famiglia nelle pubblicazioni in cui diedi l'elenco delle specie vegetali da me scoperte nell'oolite delle Alpi Venete; ma siccome io mi sentiva d'altra parte trascinato dal convincimento che in que' resti fosse rappresentato un nuovo genere di Felce, cosi v'apposi fin d'allora il nome generico di $C y$ cadopteris.

I caratteri che in quel tempo io assegnava a questo genere erano i seguenti:

"Cycadopteris. Charact. Gen. Frons pinnata vel bipinnata, pinnis vel pinnulis inte"gris, coriaceis, margine induplicatis uninerviis, in rachide decurrentibus (2) ).

Appoggiato a questi caratteri ( $\mathrm{i}$ soli che allor si potessero distinguere sui pochi esemplari rinvenuti sullo spizzo di Rotzo nei sette Comuni ed ai Pernigotti nel Vero-

(1) Nella mia memoria: Sui terreni giurassici delle Alpi Venete, pubblicatá nel 18ä2, riferii queste forme al genere Asplenites, ponendovi però in segno di dubbio un punto interrogativo.

I caratteri che mi presentarono di poi mentre confermano spettar esse alle Felci, non permettono però più di annoverarle fra le Aspleniles.

(2) Zigno. Sulle Cicadee fossili dell' oolile. Rivista periodica della R. Accad. di Padova, 18 3.

Zigno. Entdeckung, foss. Pfanzen in den Jura Gebilden der Venetischen Alpen. Neues Jahrbuch für Min. Geogn. Geol. und Petrefactenk. Herausgegeben von D.r V. Leonha rd und D. Broun. Jahrg. 1834, p. 34. 


\section{$-153-$}

nese) il chiarissimo Professore Dott. C. F. W. Braun di Bayreuth molto giustamente scorgeva nel mio genere Gycadopteris dei punti di ravvicinamento colle sue Kirchnerie ed una evidente affinità col genere Pachypteris del Brongniart (1).

Non andò guari però che posteriori osservazioni mi posero in grado di togliere questi dubbii, convertendo in certezza quanto aveva fin dalle prime sospettato. Imperciocchè, continuate per varii anni le più accurate ricerche e radunati moltissimi esemplari di queste pianticelle, ebbi la ventura di trovarne parecchi che mostravano nitidamente espressa la nervatura, ed altri su cui scorgevasi distinta nel modo più preciso la frultificazione collocata, come suole avvenire nella maggior parte delle Felci, sulla pagina inferiore della fronda.

I nuovi caratteri presentati dalle nervature e dalla fruttificazione resero allora necessaria la rifusione della frase generica per porla in accordo coi fatti che mi si svelarono di poi e che chiariscono nel modo più evidente l'appartenenza di questo genere alla classe delle Felci (2).

Ed in fatti, gli studii microscopici da me istituiti sui nuovi saggi raccolti in gran copia dopo quell' epoca mi fecero riconoscere che il margine delle pinne, anzichè essere munito di una ripiegatura, come aveva per lo innanzi indicato, è invece provveduto di una specie di orlo, lungo il quale il tessuto si mostra più consistente. Questa ingrossalura, che scorre tutt'attorno il lembo delle pinne, che persiste anche dove sia distrutto il tessuto mediano e che lascia sempre sulla roccia un'impronta più profonda del rimanente della fronda, mi fece per lungo tempo ricercare in quel punto l'esistenza della fruttificazione, che per queste apparenze era indotto a ritenere dovesse essere marginale come nelle Pteris viventi.

Le nervature, che nei primi esemplari erano appena espresse da un lieve solco longitudinale scorrente nel mezzo delle pinnule, mi si palesarono composte da un largo nervo mediano, che nell'estremità si risolve in due o tre nervicciuoli forcuti e che lateralimente è fiancheggiato da nervelti secondarii uscenti obbliquamente dal nervo principale che si biforcano una sol volta curvandosi all'infuori, ed a cui ne sono tratto tratto intercalati degli altri affalto semplici.

'Tutte queste nervature s'arrestano all' orletto marginale.

Ma il fatto piu importante e che toglie ogni dubbio sulla natura di queste pianticelle si è la scoperta dei Sori da me minutamente osșervati sopra buon numero di saggi.

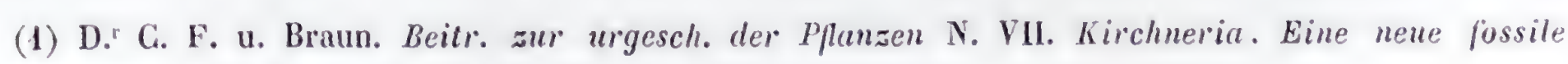
Planzengattung etc. Bayreuth 1854.

(2) Vedi Zigio. Sopra un movo genere di Felce fossile, negli Alti del R. Istituto Veneto di Scienze, Lettere ed Arti. Vol. VI. Ser. III. 1861. 
Essi presentano l'aspetto di una serie di ucchielli ricurvi, che, alquanto più in dentro del margine, stanno disposti tutt'all'intorno la pagina inferiore delle pinnule, collocati trasversalmente e paralleli ai nervi secondarii, a cui s'appoggiano per lo lungo.

Osservati col microscopio mostrano nel loro mezzo un'apertura lineare poco ed irregolarmente dilatata, il cui orifizio è guarnito di un orlo rotondato, più grosso e rilerato nel lato anteriore e più sottile nel lato posteriore (Vedi Tav. XVI. fig. 4.). Spogliato il fossile d'ogni materia calcarea mediante l'immersione nell'acido nitrico e poscia lascialo per alcuni giorni nell'acqua, potei facilmente separare le due pagine delle pinnule e riscontrare come la cuticola della pagina inferiore sia lacerata e manchi del tutto nel vano compreso dagli orli di quelle fenditure, ciò che risulta anche dall' esame dell'impronta lasciata sulla roccia dal rovescio della fronda, scorgendovisi modellate in rilievo o convesse le impressioni lasciate da queste piccole cavità.

Da queste indagini emerge chiaramente, come nella nostra Felce gli sporangi avessero origine sotto la cuticola della pagina inferiore e poscia, crescendo, la rigonfiassero e fendessero per porsi in comunicazione colla luce, cosi formando i Sori, cui nel nostro genere, tiene luogo dell'indusio l'orlo prodotto dalle labbra rigonfie dell' apertura. Questo modo di fruttificazione è assai raro nelle Felci viventi, ed abbiamo il solo genere Cryptosorus di Fee appartenente alla sezione delle Polipodiacee sporangiis immersis subcuticularibus che vi si accosti alcun poco (1).

Però nel genere Cryptosorus le aperture sorifere sono regolarmente ellittiche od ovate, hanno un contorno di eguale spessore su tutta la periferia dell'orifizio, e sono collocate sull'apice dei nerviccinoli, mentre nelle Cycadopteris le fenditure sono irregolari e ricurve, hanno l'apertura bislunga ed angusla coll'orlo più grosso nel lato anteriore, e si trovano disposte lungo i nervetti secondarii seguendone l'andamento senza oltrepassarne le estremità. Oltre a ciò, non sono tulte di un'eguale lunghezza, più lunghe riuscendo quelle poste fra i nervicciuoli e più brevi quelle che stanno in mezzo al loro biforcamento, e che perciò si arrestano all'ascella dell'inforcalura stessa (Vedi Tav. XVI. fig. 3.).

In quanto alle Felci fossili, di cui è nota la fruttificazione, non ve ne ha alcuna che al nostro genere si avvicini.

Le Kirchnerie di Braun e le Pachypteris del Brongniart, che per la forma generale dclla fronda e per la sua consistenza coriacea sembrerebbero a prima giunta potervisi accostare, non hanno fin qui svelato alcuna traccia di fruttificazione e diversificano d'altronde dalle Cycadopteris pei caratteri che si desumono dalle loro nervalure.

(1) Prof. A. L. A. Fee. Genera Filicum. Polypodiacées. Paris 1850-ă2, pag. 231. 
Nelle Kirchnerie il Prof. Braun caratterizza la nervazione nel modo seguente: Nervus medius distinctus partim obscurus; nervi secundarii flabellatim dichotomi (1): e l'illustre Brongniart distingue le sue Pachypteris colla frase: Pimmlis integris coriaceis enerviis vel uninerviis (2).

Quindi nelle prime il nervo primario in parte svanisce, ed i nervi secondarii sono flabellato-dicotomi e più fiate forcuti, come apparisce tanto dalle figure del Braun, quanto dagli esemplari ch'egli m'inviò, e nelle seconde finalmente o mancano del tutto le nervature o consistono in un sol nervo mediano. All'incontro nelle Cycadopteris il nervo principale scorrente nel mezzo delle pinuule è molto largo e rilevato e termina biforcandosi, e le nervature secondarie, pure assai marcate, sono una sol volta forcute, e di tratto in tratto intercalate da nervicciuoli semplici.

Per lutti questi caratteri io non esito a ritenere sufficientemente poste in chiaro le differenze che distinguono il genere Gycadopteris da tutti i conosciuti.

\section{Cycadopteris Brauniana Zigno.}

Tav. XVI. fig. 1, 2, 3. 4. 5. 6. Tav. XVII. fig. 1. 2.

C. Tronde simplici, lanceolata, utrinque attenuata, profunde pinnatifida lobo terminali elongato; rachide alata, elongata, basi incrassata; laciniis alternis, patulis, remotis late linearibus elongatis, integerimis, planis, apice obtusis, rolundatis, basi in rachidem alatam decurrentibus; nervo medio excurrente latissimo, apice altenuato furcalo; nervis secundariis crassiusculis obliquis, arcuatis, furcatis, interdum simplicibus; soris arcuatis, juxta nervulos dispositis.

Cycadopleris Brauniana. Zigno. Sopra un nuovo genere di Felce fossile. in Act. R. Istit. Venel. Vol. VI. Ser. III. 1861, pag. 580, Tab. IV. V. VI. fig. 1. 2. 3.; Enum. Filic. foss form. Oolith. pag. 30. - IIEn. Urwell der Schweis pag. 143, 144, fig. 96, b.

In saxo calcareo seriei oolithicae inferioris ad M. Pernigotti, ad Vallem Zuliani et ad Scandolara Provinciae Veronensis, nec non ad Rotzo et in Val d'Assa Provinciae Vicentinae. Juxta Gl. Prof. Heer occurrit etiam in stratis formationis oolithicae mediae ad Otten in Helvetia, ad Dorche prope Chanay in Gallia et ad Nusplingen in Suevia.

(1) Braun, Kirch. Loc. cit., pag. 6.

(2) Brongniart, Hist. Fégét. foss. Tom. I., pag. 166. 


\section{$-156$}

La fronda pinnatifida, elastica, coriacea e levigata di questa specie presenta nel suo generale contorno una forma lanceolato allungata costantemente semplice ed una sol volta pinnata, la quale viene sostenuta da una rachide striata scorrente lunghesso il dorso della fronda, che si prolunga alquanto inferiormente a guisa di picciuolo e si allarga rapidamente ingrossandosi verso la base.

Le pinne, o meglio lacinie, in cui si parte regolarmente la fronda, sono alterne, poco discoste tra loro, piane, perpendicolari alla rachide ed interissime. Ianno una forma ampiamente lineare allungata, ottusa e rotondata all' estremità. Misurano in larghezza per lo più dai quattro agli otto millimetri, e questo diametro si mantiene eguale in tutta la loro lunghezza, solo lievemente stringendosi verso la base ove esse si volgono obbliquamente al basso congiungendosi fra loro, e cosi formando l'ala che fiancheggia e ricopre anteriormente la rachide. La lunghezza delle lacinie che varia dai due ai quattro centimetri diminuisce gradatamente procedendo ver'so l'estremita e verso la base della fronda, ma la lacinia terminale, che ne costituisce l'apice, si prolunga e raggiunge nuovamente una lunghezza che supera alle volte del doppio quella delle due penultime lacinie laterali.

Tutto il contorno delle pinne si palesa fornito di una specie di orlo marginale più rilevato, che lascia sulla roccia un'impronta più profonda di quella lasciata dal rimanente della fronda. Quest' orlo non è prodotto da una ripiegatura del margine, come io aveva da prima creduto, ma bensi da una maggior consistenza del tessuto che, esaminato col microscopio, appare ivi tinto di un color bruno chermisino sbiadato.

Lungo ogni lacinia scorre un nervo primario che, largo alla base, si assoltiglia all'apice ed ivi si divide in due o tre nervetti forcuti. Dai lati di questo nervo principale escono obbliquamente in forma pinnata dei nervicciuoli secondarii che arcuandosi all'infuori si dirigono verso il margine terminando al limite interno dell'orlatura. Circa alla metà della loro lunghezza si mostrano forcuti, ma spesso tra i nervelti forcuti se ne osservano alcuni affatto semplici. Anche le nervature secondarie sono piuttosto grosse e ben marcate, come in proporzione assai maggiore lo è il nervo primario.

Sul rovescio di quelle frondi in cui si può scorgere la fruttificazione, essa si manifesta sotto l'aspetto di una serie di piccole fessure trasversali più o meno arcuate o ricurve, d'ineguale lunghezza, clie seguono tutto all'intorno l'andamento dei nervetti secondarii e vi si appoggiano per lo lungo. Quelle che cadono in mezzo alle dicotomie dei nervi sono meno curve e più brevi perchè s'arrestano all'ascella dell'inforcatura.

Queste fessure disposte in serie e che rassembrano gli ucchielli di una bottoniera sono prodotte dalla lacerazione della cuticola, cagionata dall' uscita degli sporangi, che nascono immersi sotto la stessa e crescendo la squarciano per aprirsi un varco e venire alla luce. 


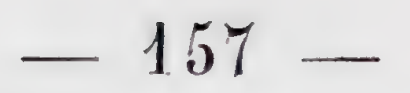

L'apertura è contornata a mo' di labbra da un orlo rotondo, rilevato, trasparente e tinto di color rubiginoso, più rigonfio nel lato anteriore e più stretto nel lato opposto e posteriore, piegato agli angoli e più soltile verso l'estremità interna ed inferiore.

Queste aperture lineari, obblique, ricurve, munite di un margine rigonfio corrispondono ai sori delle altre Felci. Mancano però dell'indusio, a cui tiene luogo invece nel nostro genere l'orlo prodotto dall'ingrossamento della lacerata cuticola.

Nel vano interposto agli orli stavano collocati gli sporangi, la cui origine subcuticulare chiaramente si comprova coll'osservazione che negli esemplari ne' quali la fruttificazione non è ancor giunta a perfetta maturità, e quindi non è ancor effettuata la lacerazione, si scorgono tante piccole prominenze disposte nello stesso senso e cagionate dal rigonfiamento della cuticola pella sottoposta spinta dei crescenti sporangi.

La forma dei sori, quale si scorge ingrandita col microscopio, è delineata alla Tavola XVI. fig. 4. $a$, $a$.

Abbonda questa specie nella calcarea giallognola spettante alla serie oolitica inferiore del M. Pernigotti presso S. Bortolamio, come pure nella Valle Zuliani presso Roverè di Velo ed a Scandolara nel Veronese. Con eguale frequenza si rinviene nella calcarea grigia di Rotzo e della Val d'Assa nel Vicentino. Il chiarissimo Professore Heer ne cita la presenza negli strati che appartengono alla serie oolitica media di Olten in Isvizzera, di Dorche presso Chanay nella Francia e di Nusplingen nella Svevia.

\section{Cycalopteris undulata Zigno.}

Tav. XVII. fig. 3. 4. 5.

C. fronde oblongo-lanceolata, profunde pinnatifida; laciniis suboppositis usque ad basim discrelis, arcuato-patulis, confertis, late linearibus elongatis, apice latioribus olstusis, rotundatis; marginibus undulato-repandis, basi plicatis, subcordato-auriculatis, in rachidem strictam alatam decurrentibus; nervo medio crasso, apice furcato; nervis secundariis obliquis, furcatis.

Cycadopteris undulata. Zigno. Enum. Filic. foss. form. Oolith. pag. 31.

In calcarea grisea seriei oolithicae inferioris ad Bienterle prope Campo Yontana in Provincia Veronensi.

La fronda di questa specie ha una forma lanceolata bislunga, assai profondamente pinnatifida e larga cinque centimetri all'incirca. Le lacinie, lunghe tre centimetri e lar- 
ghe otto millimetri, sono quasi opposte ed assai avvicinate, però separate fino al punto d'inserzione sulla rachide. Sono patenti e leggermente arcuate ed hanno una forma largamente lineare allungata, che verso l'apice insensibilmente s'allarga e termina rotondandosi. Il loro margine si palesa all'intorno irregolarmente undulato, sinuato, ed alla base della lacinia forma due pieghe laterali che danno origine a due orecchiette assai ottuse, per le quali apparisce cordato-auricolata (Vedi Tav. XVII. fig. 4.). La rachide corre ritta fino all'apice della fronda e riesce ampiamente alata mercè l'unione della base delle lacinie che in essa decorrono. Il nervo primario è poco marcato sulla pagina superiore delle lacinie, ma apparisce assai largo, rilevato e coll'apice forcuto sul dorso delle medesime, ove pure si osservano i nervetti secondarii obbliqui, robusti e forcuti; però non sempre chiaramente visibili, in causa della consistenza coriacea della fronda.

Questa specie trovata nella calcarea grigia della serie oolitica inferiore, proviene dal luogo chiamato Bienterle non lunge dal M. Alba nella Provincia di Verona.

L'arcuazione delle lacinie, l'ondulazione del loro margine e le ripiegature che ne fiancheggiano il punto d'inserzione sono i caratteri principali che distinguono la presente specie.

\section{Cycadopteris Ineterophylla Zigno.}

Tav. XVIII. fig. 1, 2, 3. 4.5.

C. fronde ovato lanceolata pinnata vel bipinnata; rachide subalata striata ; pinnis sessilibus, obliquis arcuatis vel patulis, superioribus alternis lineari-oblongis obtusis integris, mediis et inferioribus suboppositis, pinnalifidis, segmentis alternis obovato-rotundatis, brevissimis, decurrentibus, obliquis basi attenuatis connatis, terminali longiore pinnis superioribus conformi; nervis primariis excurrentibus, apice furcalis, in laciniis brevioribus dichotomis; nervis secundariis obliquis, arcuatis, furcatis, interdum simplicibus.

Cycadopteris helerophylla. Zıgno. Nuovo Gen. Fel. foss, in Act. R. Istit. Venet. Vol. VI. Sel. III. 1861, pag. 584, Tab. VIl. fig. 1.2.; Enum. Filic. foss. form. Oolith pag. 30.

In eadem formatione ad Vallem Zuliani et ad Bienterle in Provincia Veronensi et ad Rotzo et in Val d'Assa Provinciae Vicentinae. Specimen ad Tab. XVIII. fig. 4. delineatum comunicavit mihi Gl. Prof. Heer et provenit ex stratis seriei oolithicae mediae prope Nusplingen Sueviae. 
Questa specie si distingue per una fronda più espansa, ovato-lanceolata e quasi bipinnata, che verso l'estremità si mostra semplicemente pinnata 0 , per meglio dire, pinnatifida. Questa varia forma delle pinne è il carattere principale che la rende essenzialmente diversa dalle altre specie di questo genere.

Sono le pinne superiori alterne, lineari, lanceolate, allungate, interissime e rotondate all'apice, contigue e decorrenti alla base ed affatto somiglianti a quelle della Gycadopteris Brauniana.

Le medie ed inferiori quasi opposte ed assai più lunghe, talvolta obblique, spesso patenti ed arcuate, sono pinnatifide colle lacinie alterne brevissime, obovato rotondate, un po' attenuate alla base, contigue, obblique e decorrenti. Però l'ultima pinnula terminale è molto più lunga ed ha la forma lineare lanceolata allungata dalle pinne semplici superiori della fronda.

Anche in questa specie il margine delle pinne e delle pinnule mostra quella specie di orlo o contorno rilevato che si osserva nella specie antecedente, come pure è eguale la forma, la consistenza e la disposizione dei nervi, tanto nelle pinne terminali della fronda, quanto nelle pinnule allungate che si trovano all'estremità di tutte le altre pinne medie ed inferiori.

Nelle pinnule brevissime e rotondate, che fiancheggiano, per lo più in numero di quattro o sei per parte, la rachide di quest'ultime, il nervo mediano assai largo alla base apparisce monco, perchè poco sopra la sua origine si divide in tre o quattro nervetti forcuti che gli danno forma bidicotoma ed escono lateralmente presso la sua base due altri nervetti secondarii quasi sempre semplici, che s'allungano divergendo da ambi i lati del nervo principale mediano (Vedi Tav. XVIII. fig. 2.).

Nessuna traccia di fruttificazione ho potuto scorgere sui pochi esemplari di questa specie da me raccolti finora; ma la consistenza coriacea della fronda, la presenza dell'orlo marginale che ne forma il contorno, e sopra tutto la natura e disposizione della nervatura, pongono fuor di dubbio la sua spettanza allo stesso genere, di cui è tipo la precedente Gycadopteris Bramiana.

Questa specie si rinviene negli stessi strati nella Valle Zuliani ed a Bienterle nel Veronese, come pure sullo spizzo di Rotzo e nella Val d'Assa nel Vicentino. Il saggio figurato nella Tav. XVIII. alla fig. 4. mi fu comunicato dal chiarissimo Professore Heer e proviene da Nusplingen nella Svevia, ove fu rinvenuto in una formazione che si ritiene coeva del calcare scissile lilografico di Solenhofen della Baviera. 


\section{Cyeadopteris Hecrii Zigno.}

Tav. XIX. fig. 1. 1, a. 1, 6. lig. 2.

C. fronde bipinnata, rachide crassa, striata; pinnis linearibus, elongatis, obliquis; pinnulis brevibus, remoliusculis, patulis; inferioribus alternis, ovato-lanceolatis obtusis, basi angustatis; superioribus oppositis, brevissimis, obovatis, apice rotundatis, basi superne vix constrictis, inferne in rachidem decurrentibus; nervo medio crasso, apice furcato; nervulis secundariis obliquis, furcalis, interdum simplicibus.

In calcarea cinerea formationis oolithicae, ad Rotzo in Septem Communibus Provinciae Vicentinae, nec non in arenaria calcarifera lutescente, Montis Scandolara in Provincia Veronensi.

La fronda si palesa in qualche saggio decisamente bipinnata. Le pinne si diramano obbliquamente dalla rachide primaria ed hanno una forma lineare allungata della larghezza di quindici a venti millimetri. Le pinnule sono patenti ed assai brevi in confronto delle altre specie. Al punto d'inserzione sono discoste l'una dall'altra dai sei agli otto millimetri. Esse variano alquanto nella forma e disposizione, le inferiori essendo alterne, ovato-lanceolate, coll'estremità molto ottusa, e ristrette al punto d'inserzione sulla rachide secondaria che è larga un millimetro e mezzo e minutamente striata per lo lungo. La loro lunghezza sta fra i dieci e i dodici millimetri sopra una larghlezza di cinque a sei. All'incontro le superiori non giungono che a sei millimetri di lunghezza sopra tre di larghezza, sono opposte, hanno forma obovata coll'estremita rotondata e colla base lievemente ristretta nel lato superiore e decorrente nella rachide colla parte inferiore. Il nervo mediano è grosso e si divide all' apice in ramoscelli forcuti. I nervetti secondarii sono obbliqui, una sol volta forcuti ed intercalati da nervelti semplici (fig. 1. b).

Gii esemplari delineati alle fig. 1. 1, a. della Tav. XIX. derivano dalla calcaria cinerea di Rotzo nei Sette Comunị del Vicentino. Quello figurato nella stessa Tavola alla fig. 2. fu trovato nell'arenaria calcarifera giallastra del Monte Scandolara nel Veronese. Occupano queste due roccie lo stesso orizzonte geologico soggiacente al calcare ammonitico.

Questa specie, distinta dalle altre sopra tutto per la fronda bipinnata, e per la forma e brevità delle pinnule, è dedicata all'illustre Prof. D.r Osvaldo Heer di Zurigo. 


\section{$-161$}

La diversità che corre fra $\mathrm{i}$ saggi rappresentati dalle fig. 1. 1, $a$. e quello delineato alla fig. 2. potrebbe forse indurre nel sospetto che si trattasse di due differenti specie. Ma osservando accuratamente in quest'ultimo esemplare le pinnule che stanno collocate lungo la parte più bassa delle pinne, vi si scorge in modo assai palese un gradato passaggio alla forma ovato-lanceolata propria delle pinnule che guerniscono le porzioni inferiori della fronda e che si veggono disegnate alle fig. 1. 1, $a$.

Egualmente potrebbe a taluno sembrare che la Cycudopteris Heerii altro non fosse che lo stato giovane della Gycadopteris Branniana o della Gycadopteris heterophylla. Della prima ho dato la figura di una fronda appena svolta, alla Tav. XVI. fig. 6., e della seconda ho figurato una fronda giovane alla Tav. XVIII. fig. 5., e credo che l' ispezione di queste figure basterà a togliere ogni dubbio.

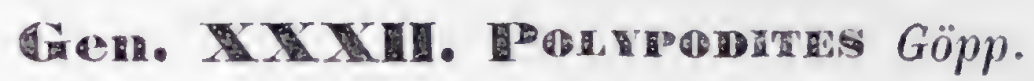

Frons simplex vel pinnata aut pinnatifida. Frons simplex subenervis. Frons pinnata, pinnis sessilibus discretis, nervo medio excurrente. Frons pinnatilida, nervis secundariis e nervo primario, angulo recto vel subacuto egredientibus, dichotomis, ramulo uno sorigero. Sori rotundi biseriales.

Görn. Sỵst. Filic. foss. pag. 175, 338 (pro parte). - Brosg. Tabl. Gen. Végét. foss. pagy. 30. - Uvg. Gen. et Spec. Plant. foss, pag. 165 (pro parte).

Come avvertii nelle osservazioni premesse alla Classe delle Felci (vedi pag. 70 della presente Opera), io adotto il genere Polypodites di Güppert, come fu circoscritto nel 1850 dal Brongniart nel suo Tableau des Genres des Végétaux fossiles, pag. 30: escludendone cioè le specie a nervi secondarj che si congiungono anastomosandosi c formando delle areole lungo il nervo primario, le quali specie hanno il naturale loro posto nel genere Phlebopteris fondato molti anni prima dallo stesso Brongniart nella sua Histoire des Végétaux fossiles. Vol. I. pag. 37\%. Accenna pure alla opportunità di questa separazione il Sig. Leckenby, nella sua Memoria sull' oolite e sulle piante fossili di Scarborough, letta alla Società Geologica di Londra nella tornata del 17 Gingno 1863 (Quart. Journ. Soc. Geol. Lond. Vol. XX. Proceed. pag. 80). 


\section{Holyodires Lindleyi Göpp.}

1. fronde profunde pinnatifida; laciniis patentissimis, linearibus elonga1is. obtusis, integerrimis, sinu rotundato interstinctis, superioribus oppositis. inferioribus alternis; nervis secundariis e nerro medio crasso, excurrente, sul) angulo recto egredientibus, dichotomis, ramulo uno furcato longiore, altero breviore sorigero; soris rotundis biserialibus.

Polypodites Lindleyi. Görp. Syst. Filic. foss, pag. 342, tab. 38, lig. 5. 6. - Momns. Caut. Brit. foss. pag. 19. - Brosg. Tabl. Gen. Végét. foss. pag. 105. - Unger. Gen. et Spec. Plant foss. pag. 167. - Zigno. Enum. Filic. foss. form. Oolith. pag. 31.

pecopteris Polypodioides. Lisd. Hut, Foss. Fl. Gr. Brit. 1. pag. 167, tab. 60 (exclus. synon.).

Steffensia Polypodioides. Presc. in Sternx. Vers. Fl. Vorw. Il. pag. 125.

Polypodium Lindleyi. Errixgsn. Farrnkr. pag. 66.

In stratis argillae calcariferae formationis oolithicae ad Gristhorpe mrope Scarborough in Anglia.

La fronda piuttosto oblunga, mostrasi profondamente pinnatifida e percorsa da una rachide liscia, avente un diametro di due millimetri. Le lacinie sono assai patenti e quasi orizzontali, alquanto fra loro discoste, e disgiunte da un seno rotondato più o meno ristretto. Le superiori sono opposte o quasi opposte, e le inferiori alterne. Sono interissime, lineari, allungate ed un poco lanceolate all'apice ove terminano ottusamente, e misurano dai quattro ai sei millimetri in larghezza, sopra una lunghezza di tre a sei centimetri. Il nervo primario scorre ritto fino all'estremita della lacinia, ed i nervetti secondarii escono da quello ad angolo retto, partendosi tosto in due rami, di cui uno più lungo si mostra furcuto coi ramoscelli assai divaricati, l'altro più breve, rimane semplice e porta nella sua estremità un soro rotondo. La disposizione poi generale dei sori risulta in serie lungo il nervo principale.

L' esemplare figurato dai Signori Lindley e Hutton, fu rinvenuto dal Sig. Bean negli strati di argilla calcarifera schistosa, spettanti alla serie oolitica inferiore, a Gristhorpe presso Scarborough nell' Inghilterra. 


\section{$-163$}

Gli autori della Flora fossile della Gran Brettagna riferirono con qualche dubbio la presente specie alla Pecopteris Polypodioides del Brongniart, Prodr. pag. 57. Ma la descrizione e la figura che di quest'ultima diede poscia il Brongniart nella sua Histoire des Végétaux fossiles, I. pag. 372, tav. 83. fig. 1., sotto il nome di Phlebopteris Polypodioides fecero chiaramente divedere che si trattava di altra specie, munita di nervetti secondarj formanti, mercè la loro congiunzione, delle areole lungo i nervi principali. La quale disposizione dei nervi indusse il Brongniart a toglierla dal genere $P e$ copteris creando per essa e per qualche altra forma analoga il genere Phlebopteris. II Göppert per evitare la confusione prodotta dall'identità del nome specifico fra due specie spettanti a generi diversi, molto opportunamente collocò quella a nervi dicotomi non reticolati nel genere Polypodites, denominandola Polypodites Lindleyi. Lo che fu poscia adottato dal Morris, dal Brongniart e dall'Unger, e con tal nome fu pure da me annoverata nella mia Enumeratio Filicum fossilium formationis Oolithicae pag. 31.

\section{Polypodites erenifolius Göpp.}

P. fronde elongata profundissime pinnatifida, rachide crassa; laciniis alternis patentissimis, lineari-elongatis, margine undulato-crenatis, apice atfenuatis oblusis, basi confluentibus, rachimque marginantibus, sinu lato truncato interstinclis; nervis secundariis e nervo medio crasso excurrente, angalo acuto egredientibus, dichotomis, ramulis furcatis, uno ante divisionem sorigero; soris rotundis, prominulis, biserialibus.

Polypodites crenifolius. Görp. Syst. Filic. foss, pag. 343. - Monns. Cat. Brit. foss. pag. 18. - Brosg. Tabl. Gen. Végét. foss. pag. 105. - Uxger. Gen. et Spec. Plant. foss. pag. 167. ZıGко. Enum. Filic. foss. form. Oolith. pag. 32.

Pecopteris erenifolia. Puilurs. Geol. Yorksh. I. pag. 148, tab. 8. fig. 11.

Pecopteris propinqua. Lisnt. Hutr. Foss. Fl. Gr. Bril. II. pag. 101 , tab. 119.

Phlebopteris propinqua. Brosc. Hist. Végét. foss. I. pag. 373, tab. 132, fig. 1.; tab. 133. lig. 2 .

Steffensia crenifolia. Presl in Stenna. Vers. Fl. Vorw. II. pag. 124

Polypodium crenifolium. Etringsn. Farrnkr. pag. 66.

In arenaria seriei oolithicae inferioris ad Gristhorpe Bay prope Scarborough, Angliae; ac in Italia in arenaria calcarifera scissili, ferruginei coloris, formationis oolithicae Montium prope Marana in Provincia Vicentina. 
In questa specie la fronda è assai profondamente pinnatifida, ed ha una rachide il cui diametro giunge ai tre millimetri, senza calcolare l'ala prodotta dal congiungimento della base delle lacinie. Quest' ultime sono alterne, molto patenti, di forma lineare allungata che si attenua leggermente verso l'apice, che è ottuso, ed hanno il margine tutto all'intorno undulato crenato. La loro larghezza sta fra $\mathrm{i}$ quattro ed i cinque millimetri, e la lunghezza raggiunge i sei centimetri. Sono distanti fra loro e separate da un ampio seno ottusamente tronco, sotto il quale si mostrano continue e formano ala alla rachide. Il nervo primario è piuttosto robusto e va dritto fino all'apice della lacinia, ed i nervetti secondarii escono obbliquamente dai suoi lati e si dicotomizzano scorrendo coi loro ramoscelli forcuti fino al margine della lacinia. I sori sono rotondi, sporgenti, e disposti in serie lungo il nervo mediano. Ognuno di essi è collocato sopra uno dei ramoscelli della prima divisione, presso alla seconda forcatura in cui è successivamente partito.

Questa specie proviene dall' arenaria scissile appartenente alla serie oolitica inferiole di Gristhorpe Bay presso Scarborough in Inghilterra. Alcuni frammenti muniti della fruttificazione furono da me trovati nella arenaria calcarifera schistosa di color ferrugineo, che forma parte dei terreni oolitici dei monti di Marana posti a settentrione di Crespadoro nella Provincia di Vicenza.

Il Sig. Leckenby attribuisce le frastagliature del margine delle lacinie ad un effetto prodotto dalla corrosione, ed esterna il parere che la presente specie altro non sia che uno stato imperfetto della Phlebopteris contigua Lrsdu. Hurr.

La regolarità delle frastagliature che rendono crenato il margine, e sopra tutto la diversa disposizione dei nervetti secondarj (che nella Phlebopteris contigua si congiungono formando le areole proprie del genere) non mi permettono di abbracciare questa opinione.

\section{Holypodites undans Göpp.}

P. fronde pinnata, rachide bisulcata flexuosa; pinnis sessilibus, alternis, patcntibus, linearibus, undulato-crenatis, acutiusculis, superficie, sulcis transrersis aequidistantibus, regulariter undulato-bullosa; nervo medio excurrente, apice evanescente, nervis secundariis dichotomis?: soris rotundis prominulis biserialibus. 


\section{$-165$}

Polypodites undans. Görp. Syst. Filic. foss. pag. 345. - Bnoss. Tabl. Gen. Végét. foss. pag. 105. - Unger. Gen. el Spec. Plant. foss. pag. 468. - Zigno. Enum. Filic. foss. form. Oolith. pag. 32.

Pecopteris undans. Jandr. Hutr. Foss. Fl. Gr. Brit. II. pag. 103, tab. 120, - Strnab. Vers.

Fl. Vorw. II. pag. 161. - Morns. Cat. Brit. foss. pag. 17.

Phlebopteris? undans. Brosag. Itist. Végét. foss. I. pag. 375, tab. 133 , fig. 3.

Polypodium undans. Etтrisgsi. Farmkr. pag. 66.

Invenitur cum praccedentibus ad Gristhorpe in Anglia. Una tantum vice mili occurrit etiam in saxo calcareo cinereo-fusco formationis oolithicae, mope Marana in Provincia Vicentina.

Si distingue questa specie per la fronda decisamente pinnata, e non bipinnatifida come fu erroneamente indicato da alcuni autori. La rachide ne è flessuosa e segnata da due solchi paralleli longitudinali, ed ha un diametro di poco più d'un millimetro. Le pime sono sessili, alterne, patenti, di forma lineare, larghe tre millimetri e lunghe all'incirca due centimetri, ondulato-crenate coll'apice piuttosto acuto. La loro superficie è segnata ad eguali distanze da solchi trasversali alquanto ottusi, che la rendono regolarmente ondulato-gibbosa, e dividono i lembi della pinna in tanti piccoli quadrati un poco obbliqui, col margine esterno rotondato e rigonfi nel mezzo, o per meglio dire convesso-concavi. I sori, che ho potuto scorgere sull'esemplare trovato a Marana nel Vicentino, sono rotondi, disposti in serie lungo il nervo mediano, e cadauno di essi sporge dalla parte convessa delle accennate divisioni. Il nervo che percorre la pinna va ritto, assottigliandosi fin presso all'apice, ove svanisce. I nervetti secondarj sono disposti allo stesso modo di quelli della Polypodites Lindleyi Göpr.

Questa specie si trova, colle precedenti, nel solito banco fitolitifero dell'oolite a Gristhorpe nell' Inghilterra. Il solo esemplare in cui m'imbattei nell' oolite delle Alpi Venete proviene dalla calcarea cinereo-bruna dei Monti di Marana nel Vicentino.

Dei nervetti secondarj non si trova alcun cenno negli autori che descrissero questa specie. In quanto poi alla fruttificazione è d'uopo ricordare come i Signori Williamson, Lindley e IIutton, Brongniart e Göppert la indicassero composta di piccoli sori puntiformi disposti trasversalmente in linee parallele lungo i lati interni delle infossature prodotte dalla rilevata ondulazione delle pinne, e come lo Sternberg Vers. Fl. Vorw. II. pag. 162, muovesse dubbio sulla natura di queste apparenze puntiformi, nè si mostrasse inclinato a vedere in esse alcun che di analogo ai sori. Il Sig. Leckenby 
$c$ il primo che abbia recentemente annunciato possedere questa specie una nervatura ed una fruttificazione analoghe a quelle della Pecopteris Polypodioides Linds. IIurt. o PoIypodites Lindleyi Göpr. (Vedi Leckenby, On the Sandst. and Sh. of the Ool. of Scarb. etc. in Quart. Journ. Soc. Geol. Lond. Vol. XX. Proceed. pag. 81).

Sull' esemplare trovato nel Vicentino non si distinguono chiaramente i nervelti, ma, come ho gia indicato, si osservano invece sulle porzioni rigonfie e sporgenti della pinna delle prominenze rotonde che hanno tutta l'apparenza di sori.

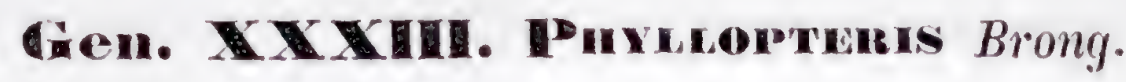

Frons simplex vel pinnata aut digitala. Pinnae lineares vel lanceolatae. Nervus medius valde notatus. Nervi secundarii obliqui, dichotomi.

Brons. Tabl. Gen. Végét. foss. pag. 22.

\section{Hyllopteris Dhillipsii Brong.}

Ph. fronde simplici vel pinnata aut digitata; pinnis lineari-lanceolatis, integerrimis, obtusis, basi apiceque angustatis; nervo medio crassiusculo excurrente, nervis secundariis tenuibus, crebris, obliquissimis, dichotomis.

Phyllopteris Phillipsii. Brong. Tabl. Gen. Végét, foss. pag. 22. - Zıgro. Enum. Filic. foss. form. Oolith. pag. 31 .

Glossopteris Phillipsii. Brong. Iist. Végét. foss. I. pag. 225, tab. 64, fig. 5. et tab. 63, fig. 2. (excl. synon. Phillips).

Taeniopteris Phillipsii. Presl in Stens. Vers. Fl. Vorw. II. pag. 140

In formatione oolithica ad Gristhorpe Cliff prope Scarborough in Comitatu York Angliae.

E molto probabile che gli esemplari figurati dal Brongniart rappresentino le pinne semplici di una fronda digitata. Le pinne sono lineari-lanceolate, ottuse all'apice, col margine interissimo ed alquanto attenuate verso le due estremità. Le percorre un nervo mediano piuttosto rilevato, che lentamente assottigliandosi giunge fino all' apice della pinna. I nervetti secondarii, numerosi, finissimi e più volte forcuti escono obbliquamente ad angolo molto acuto dai lati del nervo principale. 


\section{$-167$}

Questa specie, trovata nei terreni oolitici d'Inghilterra a Gristhorpe Cliff presso Scarborough, fu da prima fatta conoscere al Brongniart dal Prof. Phillips, che gliene inviò un disegno. Poscia il Sig. Murray procurava all'illustre antore della Histoire des Végétaux fossiles un esemplare, che è quello figurato alla tav. 61, fig. 5. dell'opera suddetta, e che non presenta alcuna traccia di nervi reticolati. Però avendola il Brongniart annoverata fra le sue Glossopteris, si riferi ad essa un'altra forma, che per la palese reticolazione dei nervetti secondarj deve invece prender posto nel genere Sagenopleris di Presl.

Göppert fu il primo che parlando della Glossopteris Phillipsii Brosa. (Acrostichites Phillipsii Göpr. Syst. Filic. foss. pag. 286) accennasse alle differenze sussistenti fra le figure date dal Brongniart e quelle che si veggono delineate sotto lo stesso nome nell'opera dei Signori Lindley e Iutton (Foss. Fl. Gr. Brit. Vol. I. tav. 63, fig. 1. 2. 3.); ed esternava in pari tempo il dubbio che potessero appartenere a specie diverse. Presl, nell'opera dello Sternberg (Vers. Fl. Vorw. II. pag. 140), andò più innanzi, e ponendo la specie figurata dal Brongniart in altro genere la descrisse solto il nome di Taeniopteris Phillipsii.

Varj anni dopo, lo stesso Brongniart nel suo Tableau des gemres des Végétaux fossiles, avrebbe tolto ogni motivo di confusione, creando per essa il genere Phyllopteris; e confermando che la pianta descritta e figurata da lui e da Phillips non ha menomamente i nervi reticolati, conclude esservi a Scarborough due piante diverse, cioè la specie presente, che serve di tipo pel genere Phyllopteris, e quella figurata dai Signori Lindley e Hutton sotto il nome di Glossopteris Phillipsii, che sarebbe una Sagenopteris molto affine a quelle del Lias di Bayreuth.

Senonchè il chiarissimo Sir C. Bunbury, nella più volte citata sua Memoria sulle Piante fossili di Scarborough, senza voler muover dubbio intorno ai caratteri assegnati dall'illustre Brongniart al genere Phyllopteris, osserva però che tal forma deve essere molto rara, non avendola giammai riscontrata nelle collezioni esistenti a York, a Scarborough e presso il Prof. Phillips, ed aggiunge che le figure dell'opera di quest'ultimo, citate dal Brongniart, non sono esatte, poichè sui disegni originali la reticolazione dei nervi secondarj è chiaramente visibile.

Fon avendo potuto esaminare gli esemplari, in mi sono attenuto, nella descrizione del genere e della specie, a quanto espose il Brongniart nel Tableau des Genres des Végétaux fossiles. Devo tuttavia avvertire che fra i sagggi di piante fossili di Scarborough che mi furono cortesemente inviati dal Sig. Leckenby, o che mi sono in altra guisa procurati, quelli che più si approssimano alla presente forma hanno tulti la nervatura reticolata ed appartengono senza alcun dubbio alla Sagenopteris Phillipsii di Presl. 


\section{Gen. XXXU. Mamana Zigno.}

Frons stipitata? digitato-radiata, vel digitato-pedata; laciniis integerrimis, basim versus attenuatis, duabus inferioribus opposilis, brevioribus. Nervi pinnati, nervulis furcatis. Frons sterilis, crassa digitato-radiata, laciniis obovalis, approximatis; nervo medio excurrente apice furcato, secundariis crebris, obliquis, arcuatis, dichotomis, tenuissimis. Frons fertilis, digitato-pedata, laciniis longioribus, divaricatis, obverse lanceolatis obtusis, basim versus angustioribus; nervo primario crasso apice furcato, nervis secundariis remotiusculis, obliquis, arcuatis, dichotomis, ramulo inferiore simplici, superiore furcato sorigero. Sori rotundi, biseriales, semiimmersi.

\section{Zıgro, Emum. Filic. foss. form. Oolith. pag. 32.}

Gli esemplari che di questo singolar genere di Felce si scopersero finora, lasciano in dubbio se fosse munito di uno stipite, dalla cui estremità allargata ed ampiamente scanalata, si espandesse la fronda, o se invece fosse composto di verticilli aperti ed abbraccianti una rachide comune a più verticilli. Nel secondo caso l'aspetto generale della fronda rammenterebbe allora quello della Trizygia speciosa di Forbes Royle; senonchè di quest'ultima non si conosce la frultificazione, e d'altronde nelle foglioline componenti i verticilli della Trizygia la disposizione dei nervi è flabellata, mentre nella Marzaria è decisamente pinnata. In questa incertezza io mi limito ad avanzare con qualche dubbio che fosse semplicemente stipitata. In quanto alla fronda, essa si divide in forma digitato-radiata o digitato-pedata, colle lacinie interissime disposte a raggio ed uscenti dal margine esterno di un sottil lembo descrivente una curva semicircolare che sembra indicare il posto ove la fronda si univa allo stipite. Le due lacinie laterali formanti le estremità della curva si distinguono per esser più piccole e più brevi delle altre. I nervi che percorrono le lacinie sono pinnati. La fruttificazione poi consta di sori rotondi, immersi per metà in una piccola cavità, e disposti in serie longitudinale fra il margine delle lacinie ed il nervo mediano, e precisamente collocati sul punto ove incomincia la forcatura di uno dei due ramoscelli in cui si dividono i nervetti secondarj. 
Due forme ho osservato in questo genere, che dapprima sospettai appartenessero a due specie diverse, e come tali le descrissi fino dal 1865 nella mia Enumeratio Filicum fossilium, alla pag. 33, sotto il nome di Marzaria Paroliniana e di Marzaria sphenophylloides. Ma ben riflettendo alle affinità che palesano, alla presenza dei sori sull' una, ed alla mancanza di ogni traccia di fruttificazione sull'altra, come pure alla diversa consistenza della fronda, che svela un'analogia con quanto avviene in alcune Ielci viventi, in cui le pinne o le frondi fertili si mostrano per lo più emaciate in confronto delle sterili, mi trovo ora inclinato a considerarle quali stati diversi rappresentanti l'uno la fronda sterile, e l'altro la fronda fertile di una medesima specie.

Alieno dall'introdurre nomi nnovi nella scienza avrei ben volentieri comprese queste forme in alcuno dei generi conosciuti, se dopo le più accurate indagini non mi fossi convinto che nessuno vi corrisponde. E per vero giova qui rammentare, come nelle Felci fossili, la forma digitata della fronda sia per lo più accompagnata da uma nervatura, o flabellata (Cyclopteris), o più o meno areolata (Bajera, Thaumatopteris, Camptopteris, Clathropteris, Sagenopteris). I generi Andriania e Gutbiera, i cui nervi non formano delle areole, li hanuo però disposti in modo affatto diverso da quelli dellat Marzaria. Il solo genere Laccopteris, ove non avesse le pinne profondamente pinnatifide, si accosterebbe alcun poco al presente, per un'analoga distribuzione dei nervi nelle lacinic.

Egualmente nelle Felci della Flora attuale veggiamo le frondi flabellate, digitate, pedate o palmate, fornite o di nervi eguali flabellati (Acrostichum peltatum Swrartz, Acrostichum sphenophyllum Kunze, Actiniopteris australis Lnк, Actiniopteris radialu Lans), oppure di nervi reticolati (Pteris pedata Lnx., Pteris hederacea Prese, Gymnogramme palmala Lisk, Cheiroglossa palmata PrEsL), e soltanto fra le Ligodiacee la forma palmato-lobata della fronda si trova associata alla distribuzione pinnata dei nervi nel Lygodium palmatum di Swantz, Felce rampicante dell'America Settentrionale. Però nel nostro genere i nervi sono assai meno numerosi e molto più grossi, e la frultificazione poi è affatto diversa da quella del genere Lygodium.

Altre affinita potranno forse esserci svelate in seguito dalla forma e disposizione degli sporangi, dei quali finora non potei rilevare alcuna traccia per quanto abbia diligentemente esaminato col microscopio la sostanza polverosa contenuta nelle piccole cavita sorifere.

Ho dedicato questo genere alla memoria del rinomato geologo Conte Giuseppe Marzari Pencati. Chi bramasse conoscere di quanto sia debitrice la scienza a questo illustre Vicentino ed in quanta onoranza fosse tenuto dagli uomini più celebri che al suo tempo collivassero la Geologia e la Mineralogia, ne legga la Biografia pubblicata nel 1836 (Bibl. Ital. Vol. 83) dal chiarissimo geologo ed amico suo Commendatore Lodovico Pasini Senatore del Regno. 


\section{Marzaria Daroliniana Zigno.}

Tav. XIX. fig. 3. 3, a. 3, 6. fig. 4.5.6.7

M. fronde stipitata? digitato-radiata aut digitato-pedata, lacinis inacqualibus, integerrimis, duabus inferioribus brevioribus: fronde sterili, crassa digitato-radiata, laciniis octo, approximatis, contiguis, obovatis, apice rolundatis, basi altenuatis; nervo medio tenui, apice furcalo, nervulis secundariis crebris, arcuatis, furcatis, tenuissimis: fronde fertili, digitato-pedata, laciniis duodecim, longioribus, divaricatis, obverse lanceolatis, obtusis, basim versus allenualis et angustioribus; nervo medio crasso canaliculato, excurrente apice furcato, nervis secundariis crassiusculis, oblique e nervo medio exeuntibus, arcuatis, dicholomis, ramulo superiore furcato sorigero, inferiore simplici. Soris rolundis, biserialibus semimmersis.

Marzaria Paroliniana. Zigno. Eumm. Filic. foss, form. Oolith. pag. 32 (Frons ferlilis).

Marzaria sphenophylloides. Zigio. Loc. cil. pag. 33 (Frons sterilis).

In stratis calcareis seriei Oolithicae inferioris ad Vallem Zuliani prope Roverè di Telo in Provincia Veronensi.

Questa curiosa Felce ci presenta due forme che descrissi fino dal 1865 come specie diverse, ma che ora attribuisco l' una allo stato sterile, l'altra allo stato fertile della medesima specie. Il seno semicircolare più o meno aperto che occupa il mezzo della fronda, ma ne forma realmente la base, sembra indicare il punto ov'essa si univa allo slipite, che però, nei varii saggi raccolti finora, non è visibile.

La forma digitato-radiata (Tav. KIX. fig. 6. T) che apparisce quasi verticillata pella piccolezza del seno mediano, è quella che ritengo appartenere alla fronda sterile. Essa e. munita di otto lacinie ineguali, interissime, di consistenza crassa, di forma obovata, rotondate all'apice ed attenuate presso la base, ove in qualche esemplare si mostrano quasi cuneiformi. Sono molto tra loro avvicinate e per lo più contigue nel terzo inferiore, e le due ultime, che si protendono dalle estremità del seno formato dalla riunione delle basi delle lacinie, sono assai più piccole delle altre, misurando sette millimetri in lunghezza e poco più di tre in larghezza, mentre le rimanenti arrivano ai dodici millimetri di lunghezza sopra una larghezza di cinque. La loro consistenza piuttosto crassa, rende meno apparente la nervatura pinnata che le percorre. Si distingue però un nervo mediano alquanto sottile, che verso l'apice svanisce risolvendosi in più 
nervelti forcuti, e dai lati di quello escono numerosi ad angolo acuto i nervetti secondarj, soltilissimi, che poscia facendosi arcuati e biforcandosi, volgono verso il margine delle lacinie. La fronda fertile all'incontro più irregolare e sparpagliata (Tav. XIX. fig. 3. 4.) ha una forma digitato-pedata, col seno della base più aperto, e si divide in dodici lacinie lunghe sedici nillimetri e larghe, nella parte loro piu ampia, dai tre ai cinque millimetri. Anche nella fronda fertile le due ultime lacinie laterali inferiori sono più piccole non oltrepassando i nove millimetri in lunghezza ed i tre in larghezza. La forma delle lacinie è lanceolato-oblunga, coll'apice ottuso e gradatamente attenuata verso la base. Sono pure ineguali e col margine interissimo, giammai contigue, ma piuttosto distaccate fra loro e divaricate. Il nervo mediano da cui sono percorse è molto marcato, grosso e scanalato e scorre fino all' apice ove diventa forcuto. I nervetti secondarj sono robusti, meno numerosi di quelli che si osservano nella fronda sterile e dicotomi, col ramoscello inferiore semplice ed il superiore forcuto (Tav. XIX. fig. 3. a fig. 5.).

La fruttificazione si palesa composta di sori rotondi, disposti in serie longitudinale tra il nervo mediano ed il margine della lacinia e collocali ove comincia la forcatura del ramoscello superiore dei nervetti secondarj (Tav. XIX. fig. 3. 6.). Levando colla punta di un ago la sostanza polverosa che occupa il posto degli sporangi, si scorge una piccola cavità in cui apparisce fosse immersa la base del soro. Degli sporangi non ho pointo finora trovar traccia alcuna.

Tutti i saggi di questa specie provengono dagli strati calcarei superiori della serie oolitica inferiore e si rinvennero nella Valle Zuliani presso Roverè di Velo nella Provincia Veronese.

\section{Ordo XiII. DigTyopterideae.}

Frons simplex vel digitala, pinnatilida aut pinnata vel bipinnata; nervis aut omnibus areolato-reticulatis, nervo medio evanescente, vel primariis rectis excurrentibus, aut bi-dichotomis, secundariis vero reticulatis, aut angulo recto vel subrecto a nervo medio cxeuntibus dichotomis, ramulis in areolas polygonalcs confluentibus. Fructificatio, soris rotundis biserialibus. sporangiis stellatim dispositis.

Zigno, Emum. Filic, foss, form. Oolith, pag. 33. 


\section{$-172$}

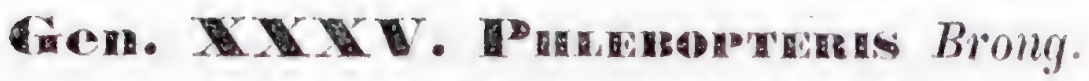

Frons profunde pinnatifida, laciniis integerrimis. Nervus medius rigidus excurrens. Nervi secundarii dichotomi apice liberi, ramulis inferioribus plus minusve arcuatim anastomosantibus. areolas polygonales, nervum medium rachimque marginantes, efformantibus. Sori rotundi biseriales, sporangiis $:-8$ stellatim dispositis.

Brong. Hist. Végét. foss. I. pag. 371; Tabl. Gen. Végét. foss. pag. 30. - Strnn. Vers. lil. Vorw. II. pag. 163.

Hemitelites. Görp. Syst. Filic. foss. pag. 329 (pro parte). - Uxger. Gen. el Spec. Plame. foss. liag. 160 (pro parte).

\section{Plabebopteris polypodiondes Brong.}

Phl. fronde profunde pinnatifida, laciniis linearibus elongalis, integerrimis, obtusis, remotis, basi confluentibus rachimque marginantibus, sinu obtuso, rotundato, interstinclis; nervo medio recto excurrente; nervis secundariis angulo subrecto e nervo medio egredientibus dichotomis, ramulis transversim conjunctis, areolas subtetragonas rachim et nervum medium marginantes, efficientibus, externe nervulos e basi bifidos emiltentibus; soris rolundis, biserialibus, extra areolas insertis.

Phlebopleris polypodioides. Brong. llist. Végét. foss. I. pag. 372, tab. 83, fig. 1. - Stennw. Vers. Fl. Voru. II. pag. 163. - Zigno. Enum. Filic. foss. form. Oolith. pag. 33.

Pecopteris polypodioides. Влолg. Prodr. pag. 57.

IIemilelites polypodioides. Görr. Syst. Filic. foss. pag̨. 336, lab. 15, fig. 8. 9

Hemitelia polypodioides. Ertingsn. Farrnkr. pag. 226.

In formatione oolithica prope Scarborough Angliae, nec non in calcarea cinerea seriei oolithicae inferioris ad Val d'Assa in Provincia Vicentina.

L'esemplare figurato nell' Opera del Brongniart ed in quella del Göppert ci presenta una porzione di fronda profondamente pinnatifida colla rachide principale liscia, dritta ed avente il diametro di poco più d'un millimetro e mezzo. Le pinnule o laci- 


\section{$-173$}

nie, lunghe all'incirca tre centimetri e mezzo e larghe dai quattro ai cinque millimetri sono lineari allungate, interissime, ottuse all' apice e fra loro parallele. Sono esse un po' distanti fra loro, ma confluiscono alla base formando larga ala alla rachide e sono disgiunte da seni ottusi o rotondati. Il nervo primario che le percorre nel mezzo corre ritto fino all' apice, e dai suoi lati escono ad angolo quasi retto dei nervetti secondarj appajati che divergendo fra loro si uniscono alle paja vicine col mezzo di ramoscelli trasversali e formano cosi, lungo il nervo mediano ed ai lati della rachide, delle areole quadrilatere piuttosto regolari, dal cui lato esterno sorgono dei nervelti di terz'ordine bifidi fin dalla base, coi rami semplici alcun poco divergenti e talvolta anche forcuti che scorrono fino al margine della lacinia. La fruttificazione è composta di sori minuti e rotondi disposti in serie longitudinale lungo il nervo mediano e collocati all'infuori delle areole, ove alternano coi nervetti di terz'ordine.

Questa specie fu trovata nel deposito fitolitifero speltante alla formazione oolitica che si osserva presso Scarborough nell'Inghilterra. Io ne ho trovato dei frammenti colla fruttificazione nella calcarea grigia della Val d'Assa nei Sette Comuni del Vicentino, roccia che appartiene alla serie oolitica inferiore.

\section{Holleldopteris contiogna Lindl. Hutt.}

Phl. fronde profunde pinnatifida, laciniis lanceolatis integerrimis, sursum subcurvatis, apicem versus altenuatis obtusis, basi sensim latioribus, confluentibus approximalis, sinu angustissimo discretis; nervis secundariis angulo subrecto e nervo medio egredientibus, dichotomis, ramulis transversis arcualim conjunctis, areolas obliquas irregulariter subcordato-hexagonoideas, nervum medium rachimque marginantes efficientibus, externe nervulos simplices, rarissime apicem versus furcatos, emittentibus.

Phlebopteris contigua. Lirnu. Hutr. Foss. Fl. Gr. Brit. II. pag. 177, 178, lab. 144:- Stenns. Vers. Fl. Vorw. II. pag. 166. - Zigno. Enum. Filic. foss. form. Oolith. pag. 34.

Hemitelites Brownii. Göer. Syst. Filic. foss. pag. 334, tab. 38, fig. 1. - Ung. Gen. et Spec. Plant. foss. pag. 161.

Hemitelia Brownii. Eтtuxgsm. Farmk. pag. 226.

In globulis ferruminosis formationis Oolithicae ad Gristhorpe Bay prope Scarborough in Anglia, nec non in stratis calcareis seriei Oolithicae inferioris Montium Marana in Provincia Vicentina. 


\section{$-174$}

La fronda di questa specie è pure profondamente pinnatifida, colle lacinie interissime e confluenti alla base, ma assai più avvicinate e separate soltanto da un seno angustissimo. Ilanno eziandio la base più larga e vanno gradatamente attenuandosi verso l'apice curvandosi alquanto all'insù. Nella parte più ampia che è l'inferiore giungono ai dodici millimetri di larghezza e misurano talyolta in lunghezza i cinque centimetri. La rachide è minutamente striata per lo lungo ed ha un diametro di circa tre millimetri che va rapidamente assottigliandosi verso l'estremità. Il nervo primario scorre ben marcalo fino all'apice delle lacinie, ed i nervetti secondarj ne escono quasi ad angolo retto, tosto dividendosi in forma dicotoma e mettendo dei ramoscelli trasversali che arcuandosi li congiungono e formano, lungo la rachide ed il nervo mediano, delle areole obblique irregolarmente cordato-esagone, dal cui lato esterno escono dei nervetti semplici e paralleli, rade volte coll' estremità forcuta.

L'esemplare descritto dal Sig. Williamson e figurato dai Signori Lindley e IIutton fu trovato in un nodulo ferruginoso nella formazione oolitica a Gristhorpe Bay presso Scarborough nell'Inghilterra. Anche di questa specie ho trovato dei frammenti nella formazione oolitica delle Alpi Venete, nella calcarea grigio-bruna dei Monti di Marana nel Vicentino.

Questa specie è diversa dalla precedente non solo per avere le lacinie più larghe e più avvicinate alla base e maggiormente attenuate verso l'apice, ma sopra tutto per la forma irregolarmente cordato-esagona ed obbliqua delle areole, e per la semplicitit ed il parallelismo dei nervetti di terz' ordine che escono equidistanti dal lato esterno delle areole siesse, e che assai di rado si mostrano forcuti verso l'estremità; mentre nella Phlebopteris polypodioides Brosg. le areole, come vedemmo, sono regolari tetragone, ed i nervetti di terz'ordine che si diramano dal loro lato esterno, sono bifidi fin dalla base, un po'divergenti e spesse volte con uno dei due rami forcuto.

La nervatura di questa specie presenta una qualche analogia con quella della Phlebopteris Schouwii del Brongniart.

\section{Inlebopteris oodwardii Leckenby.}

Phl. fronde.... pinnis pinnulisve linearibus, angustis, elongatis; nervo medio crasso excurrente longitudinaliter bisulcato; nervis secundariis angulo recto exeuntibus, basi simplicibus horizontalibus, remotis, marginem rersus flabellatim bi-trifurcatis, ramulorum lateralium conjunclione, areolas 


\section{$-175-$}

pentagonas efficientibus; soris stellatis, biserialibus, in areolarum centro, solitariis.

Phlebopteris Woodwardii. Lecnisnx. On the Sandst and sh. of the Ool. of Scarb. will descrip. of Foss. Pl. in Quart. Journ. Geul. Soc. Lond. Vol. XX. Proceedings pag. 81, lab. VIII. fig. 6 .

Polypodites Woodwardii. Zicio. Enum. Filic, foss. form. Oolith. pag. 32

In saxo calcareo-arenaceo scissili, seriei Oolithicae inferioris, ad Cloughton mope Scarborough Angliae.

II frammento figurato dal Sigg. Leckenby con un ingrandimento di tre diametri non permette che si pronunci alcum giudizio sulla forma e disposizione della fronda, nè manco puossi arguire se sia porzione di una pinna o piutlosto di una pinnula.

Sia che all' una od all'altra appartenga, esso si presenta di forma lineare angusta, che pel parallelismo dei margini si può ritenere fosse alquanto allungata. Il nervo mediano corre dritto per tutta la lunghezza del frammento, è piuttosto rilevato ed appariscente e segnato longitudinalmente da un solco per parte. I nervi secondarj escono ad angolo retto dal nervo primario, si mantengono semplici ed orizzontali fino alla metà, e poscia si dividono in ramoscelli due o tre volte forcuti, che espandendosi a ventaglio vanno a metter capo nel margine. I ramoscelli laterali si congiungono con quelli dei nervi vicini e formano delle areole regolari pentagone interposte ai nervi secondarj e fiancheggianti il nervo mediano. La frultificazione si palesa composta di piccoli sori aventi una forma stellata, cadauno dei quali è collocato nel centro delle areole. formando cosi due serie Iongitudinali disposte una per parte del nervo principale.

Questa specic fu trovata dal chiarissimo Sig. Leckenby nelle arenarie calcarifere inferiori dulla formazione oolitica a Gloughton presso Scarborongh nell' Inghilterra.

Nella figura che di questa nuova specie ci porge il Sig. Leckenby le areole appariscono aperte verso il margine della pimna, non essendovi minimamente espressa la congiunzione dei ramoscelli laterali, in cui sono divisi i nervi sccondarj. E fu per tal ragione che nella mia Emumeratio Filicum fossilium formationis Oolithicae trasportai questa forma nel genere Polypodites, arendo circoscritio il genere Phlebopteris a quelle sole specie che son provvedute di vere areole perfettamente chiuse, come svolsi anche nell'opera presente alla pag. 7 , nelle osservazioni generali premesse alla Classe delle Felci. Ma dopo la stampa di quel foglio mi giungeva lettera del Sig. Leckenby nella quale mi faceva avvertito che il disegno da lui pubblicato non era in quella parte 
esatto, mentre le areole sull' esemplare scorgevansi realmente chiuse. Ciò ebbi pur campo di verificare sopra un saggio gentilmente favoritomi di poi dallo stesso Sig. Leckenby, per cui cessa ogni molivo di dubbio sulla appartenenza di questa specic al genere Phlebopteris in cui fu posta dallo scopritore.

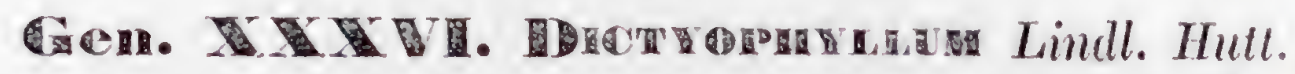

Frons pinnatifida vel pedato-pinnatilida. Laciniae pinnatifidae vel inciso-lobatae aut crenatae. Laciniarum nervi primarii excurrentes. Nervi secundarii angulo subrecto egredientes, flexuose excurrentes, apice dichotomo-reticulati. Nervi tertiarii. angulo recto, egredientes pluries dichotomi, ramulis divaricatis anastomosantibus, areolas pluriscriales inaequaliter hexagonoideas vel pentagonas elficientibus; nervulis postremis in areolas minores irregulariter polygonales confluentibus. Sori rolundi sparsi. Sporangia annulo multiarticulato instructa. Sporac tetraedro-globosae.

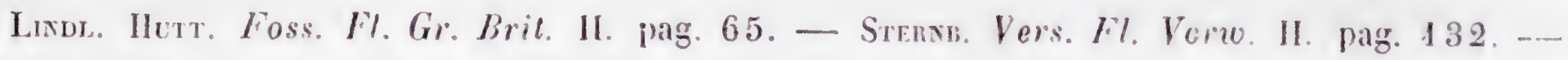
Schexr, Foss. Fl. d. Grenzsch. d. Keup. und Lias Frank, pag. 75.

Diplodiclyon. Braux in Münster. Beitr. z. Petrefact. Vi. pag. 13. - Brosg. Tabl. Gen. Tegut foss. pag. 33. - Ung. Gen. et Spec. Plant. foss. pag. 164.

Polypodites spec. Göpr, et Ung. - Camptopteris spec. Prest. et Brosr.

\section{Dietyophyllam rugosum Lindl. Hutt.}

Tav. XXIII. fig. 2, fig. 2, a.

D. fronde profunde pinnatifida, rachide crassa striata; laciniis alternis, distantibus, patulis, oblongo-lanceolatis, apice acuminatis, basi confluentibus rachimque late marginantibus, sinu lato, subtruncato, inacqualiter snuato dentato, discretis; marginibus, grosse dentato-lobatis, lobis subovatis acutiusculis obtusisve; nervis secundariis, e nervo medio tereti excurrente, angulo subrecto egredientibus, nunc flexuose versus apicem loborum (xcurrentes, nunc, ante apicem, dichotomo-reticulatis; nervis tertiariis, angulo recto egredientibus, pluries dichotomis, ramulis divaricatis, anastomosanti- 


\section{$-177-$}

bus, cum prioribus areolas pluriseriales, inaequaliter hexagonoideas vel pentagonas, efficientibus; nervulis postremis, in areolas minores irregulariter polygonales, confluentibus; soris rotundis, sparsis.

Dictyophyllum rugosum. Lind. Пuтt, Foss. Fl. Gr. Brit. II. pag. 65, tab. 104. - Stenni. Vers. Fl. Norw. II. pag. 133. - Monns. Cat. Bril. foss. pag. 7.

Phyllites nervulosus. Phillirs. Geol. Yorksh. I. pag. 148, tab. 8. fig. 9.

Phlebopteris Phillipsii. Bnosg. Hist. Végét. foss. I. pag. 377, tab. 133, fig. 1. - Ztgro. Enum. Filic. foss. form. Oolith. pag. 34 .

Polypodites heracleifolius. Görp. Syst. Filic. foss. pag. 344. - Uxaer. Gen. el Spec. Plant. foss. pag. 167.

Camptopteris Phillipsii. Brong. Tabl. Gen. Végét. foss. pag. 105.

Polypodium heracleifolium. Etringsir. Farrnkr. pag. 67.

In arenaria calcarifera inferiori formationis oolithicae, ad Gristhorpe et ad Cloughton prope Scarborough in comitatu York, Angliae. Juxta Cl. Prof. Unger occurrit etiam ad IInterholz prope Waidhofen, nec non ad Kirchberg et Pechgraben, Austriae.

La fronda ampia e profondamente pinnatifida è percorsa da una rachide avente il diametro di tre millimetri e leggermente segnata da minute strie longitudinali. Le lacinie in cui è frastagliata, sono alterne, disianti e patenti, di forma lanceolata bislunga, piuttosto allungate ed acuminate verso l'apice; confluenti alla base ove formano larga ala alla rachide disgiunte fra loro da un ampio seno, ottusamente tronco, il cui margine per lo più intero è talvolta sinuato-dentato. All'incontro i margini delle lacinie sono largamente dentato-lobati coi lobi quasi ovati ed un poco acuti, ma spesso anche ottusi e rotondati. La larghezza delle lacinie varia fra $\mathrm{i}$ venti ed $\mathrm{i}$ ventiotto millimetri, e la lunghezza, per quanto si può desumere dagli esemplari esistenti, deve avere raggiunto gli otto centimetri e forse più. Il nervo primario è liscio e rotondo, e scorre fino all' apice della lacinia. I nervi secondarj escono da quello ad angolo quasi retto, alcuni scorrendo in modo flessuoso verso la estremità dei lobi, altri dividendosi in forma dicotoma ed anastomosandosi assai prima di giungervi. Escono da questi ad angolo retto dei nervetli che diremo terziarj, i quali si dicotomizzano più volte in ramoscelli divaricati che anastomosandosi e suddividendosi formano cogli antecedenti più serie di maglie poligone di cui le maggiori, irregolarmente esagone o pentagone, fiancheggiano per lo più il nervo primario. Le ultime ramificazioni dei nervicciuoli confluiscono anche esse fra di loro e formano delle areole più piccole pure irregolarmente poligone. Tav. XXIII. fig. 2., $a$. 


\section{$-178-$}

Della fruttificazione di questa specie non è fatta menzione in alcun autore. Però sopra un esemplare della mia collezione, si scorgono qua e là delle piccole prominenze rotonde, che sembrano corrispondere ai sori, osservati dal chiarissimo Prof. Schenk di Wirzburgo, sopra altre specie di questo genere.

Questa specie si rinviene nelle arenarie calcarifere scistose della serie oolitica inferiore, tanto a Gristhorpe che a Gloughton presso Scarborough nell' Inghilterra. Sccondo il chiarissimo Prof. Unger trovasi pur anco ad Hinterhol presso Waidhofen ed a Kirchberg e Pechgraben in Austria.

Quasi tutti gli autori che descrissero e figurarono la presente specie si attennero alla figura ed alla descrizione che ne diedero i Signori Lindley e Hutton nella Fossil Flora of Great Britain loc. cit., ommettendo di dare la dovuta importanza alla reticolazione dei nervi. Il solo Brongniart si espresse in modo più esplicito ponendola nel genere Phlebopteris e rilevando che i ramoscelli dei nervetti secondarj si anastomosano fra di loro (Tedi Hist. Végét. foss. loc. cit.). Più tardi, in quell'aurea Operetta che è il suo Tableau des genres des Végétaux fossiles, alla pagg. 105, egli la annoverò nell' elenco delle piante dell' oolite sotto il nome di Camptopteris Phillipsii, con ciò dando a divedere come meglio di ogni altro si fosse accostato al vero circa alla distribuzione dei nervi. Però questa specie si distingue anche dal genere Camptopteris principalmente per la diversa forma delle areole primarie e per una più irregolare disposizione dei nervi secondarii, come si scorgerà di leggieri dalla descrizione che ne ho dato. Recentemente il Prof. Schenk di Würzburg nella sua bell'opera intitolata: Die Fossile Flora der Grenzschichten des Keupers und lias Frankens, pag. 75, 76, fece invece conoscere le analogie sussistenti fra la nervatura del genere Dictyophyllum Lindu. Hutr. e quella del genere Diplodictyon di Braun, e riuni questi due generi conservando la prima denominazione come la più antica. Gli esemplari del Dictyophyllum rugosum Lrsd. Iutr. che posseggo nel mio Gabinetto e sui quali si può chiaramente distinguere i diversi ordini de' nervi e le varie forme delle areole confermano pienamente le osservazioni del Prof. Schenk.

\section{Dietyophyllum Leckenbyi Zigno.}

Tav. XXIII. fig. 1. 1, a.

D. fronde profundissime pinnatifida, rachide striata; laciniis alternis, aequalibus, remotis, horizontaliter patentibus, late linearibus elongatis, obtu- 


\section{$-179$}

se remoteque undulato-crenatis, apicem versus sensim attenuatis, basi abrupte dilatatis rachimque marginantibus, sinu latissimo truncato integerrimo discretis; nervo medio tereti excurrente, nervis secundariis angulo recto egredientibus, dichotomis, ramulis in areolas inaequaliter pentagonas vel hexagonoideas subarcuatim anastomosantibus, nervulis omnibus in areolas minores irregulariter polygonales confluentibus.

Invenitur cum praecedenti in formatione oolithica prope Scarborough in Anglia.

In questa specie la fronda è assai più profondamente pinnatifida ed è percorsa da una rachide più sottile ed appena segnata da lievissime strie longitudinali, che non possono chiaramente vedersi senza l'ajuto della lente. Le lacinie in cui è partita sono eguali, molto discoste l'una dall'altra, alterne ed orizzontali; di forma lineare allargata, coi margini ottusamente e largamente ondulato-crenati, o per meglio dire colle crenature molto larghe, appianate e poco profonde in guisa che danno un'apparenza leggermente ondulata al margine. Esse si attenuano molto lentamente verso l'estremitì, mentre alla base si dilatano bruscamente e fondendosi colle altre formano l'ala della rachide. Poco sopra la base sono larghe un centimetro e raggiungono in lunghezza i dieci centimetri. L'ampio seno che le disgiunge è di forma tronca cogli angoli ottusi e col margine intero. Il nervo mediano delle lacinie le percorre fino all'apice. I nervi secondarii escono ad angolo retto dal nervo mediano e poco sopra la loro origine si dicotomizzano in ramoscelli talvolta legggermente arcuati, i quali si congiungono fra di loro e formano delle areole irregolarmente pentagone od esagone che fiancheggiano il nervo mediano. Da questi ramoscelli, altri nervetti si diramano in forma dicotoma, i quali anastomosandosi danno origine a delle areole minori irregolari e poligone.

Il saggio di questa bella specie proviene dal solito deposito di piante fossili che giace nella formazione oolitica della Contea di York nell'Inghilterra, e mi fu favorito dal più volte lodato chiarissimo Sig. Leckenby di Scarborough.

Questa forma che mi giunse dall' Inghilterra unitamente a varii saggi bene caratterizzati del Dictyophylllum rugosum Linds. Iuтt. merita di esserne separata tanto pelía diversità che presenta il suo aspetto generale quanto pegli altri caratteri che ho già notato nella descrizione. In confronto del Dictyophyllum rugosum L. If. essa ha la fronda più regolarmente pinnatifida, gli spazj interposti alle pinne o lacinie più ampi e circoscrilli presso la rachide da seni quasi rettangolari, il margine che fiancheggia 
quest' ultima è quasi dritto o leggermente flessuoso, le pinne o lacinie sono più strelte e più patenti anzi orizzontali, molto più allungate, e gli orli anzichè essere frastagliati in lobi piuttosto profondi e talvolta acuti, sono invece appena segnati da larghe crenature molto appianate e poco profonde per cui il margine apparisce soltanto lievemente ondulato.

La grandezza dell' esemplare e la regolare costanza di questi caralteri in ogni parte del medesimo m'indussero a ritenere questa specie come affatto distinta dalla precedente.

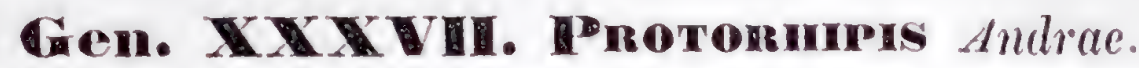

Frons semiorbiculata. Nervi primarii flabellati vel radiatim dispositi, pluries dichotomi. Nervi secundarii transversales cum prioribus areolas parallelogrammas efficientes, ramulis in areolas subquadratas confluentibus.

Axdrae. Foss. Fl. Siebenb. und Banal. pag. 35.

\section{Protorhipis Asarifolia Zigno.}

Tav. IX. fig. 2, 2, a.

P. fronde stipitata? orbiculato-reniformi convexiuscula, margine integerrimo; nervis primariis radiatim dispositis, crassiusculis, subflexuosis, pluries dichotomis, ramulis divaricatis; nervis secundariis tenuibus, ramulis in areolas polygonales subquadratas confluentibus.

Protorhipis Asarifolia. Zıgo. Enum. Filic. foss, form. Oolith. pag. 34 .

In saxo calcareo cinereo seriei oolithicae inferioris ad Vallem Zuliani prope Roverè di Velo in Provincia Veronensi.

La fronda di questa curiosa specie è orbiculato-reniforme colla superficie leggermente convessa, e col margine interissimo. E molto probabile che fosse sorretta da uno stipite scorgendosi nel seno mediano una frattura che presenta l'apparenza del taglio trasversale di uno stipite scanalato. Da quel punto si espandono a raggio cinque o sei nervi principali bene appariscenti, un po'flessuosi e divaricati, più volte dicotomi, colle dicotomie allargate che si ripetono fino al margine della fronda. Da questi si diramano 


\section{$-181$}

ad angolo retto i nervetti secondarj molto più sottili, i cui ramoscelli tenuissimi confluiscono reticolandosi in guisa da formare delle areole poligone per lo più irregolarmente quadrate.

L'unico saggio di questa specie che mi fu dato osservare proviene dalla calcaria grigia spettante alla serie oolitica inferiore della Valle Zuliani presso Roverè di Velo nella Provincia Veronese.

\section{Gen. XXXVII. Canptopteris Presl.}

Frons subcrassa rigida pedato-pinnatifida. Nervi primarii prominentes, crassi, excurrentes. Nervi secundarii obliqui subflexuosi usque ad marginem frondis producli, ramosi, ramulis angulo recto egredientibus, subarcuatis, transversim in arcolas plus minusve regulariter parallelogrammas confluentibus. Venulae ramosissimae, in areolas irregulariter quadratas, vel parallelogrammas, aut subhexagonas, confluentes, rarius libere desinentes.

Presl in Stensis. Vers. Fl. Vorw. II. pag. 168. - Göpr. Gall. foss. P 7 . fasc. 5. 6. pag. 149. - Brong. Tabl. Gen. Végél. foss. pag. 31. - Urg. Gen. el Spec. Pl. foss. pag. 162.

\section{Camptopteris jurassica Göpp.}

C. fronde...., laciniis lanceolato-oblongis, remote sinuato-dentatis, dentibus obtusis; nervo primario crasso subflexuoso excurrente; secundariis suboppositis, obliquis, aeque distantibus, usque ad laciniarum marginem excurrentibus, ramulis transversis, simplicibus, subflexuosis, aeque distantibus, unitis, areolas plus minusve regulariter parallelogrammas efficientibus; venulis crebris, in areolas regulares, subhexagonas confluentibus.

Camptopteris jurassica. Görr. Foss. Fl. d. m. jurag in Uebers. d. Arbeit. d. Schles. Gesellsch. 1845-46, pag. 143; Gall. foss. Pfl. fasc. 3. 4., tab. 17, fig. 4. - Uxg. Gen. et Spec. Pl. foss. pag. 162. - Zigno. Enum. Filic. Joss, form. Oolith. pag. 33.

Aspidium jurassicum. EtTixgsı. Farrnkr. pag. 201. 


\section{$-182-$}

In argilla ferruminosa formationis Oolithicae ad Matzdorf Silesiae, nec non in saxo calcareo ferruginei coloris seriei oolithicae inferioris montium prope Marana et Crespadoro in Provincia Vicentina.

Il frammento figurato dal Prof. Göppert (ma non descritto) nell'Opera: Die Gattungen der fossilen Pflanzen alla Tav. XVII. fig. 4. dei fascicoli 3. 4., non giunge a dare un'idea della forma generale della fronda, ma rappresenta porzione di una delle lacinie in cui era probabilmente frastagliata. La lunghezza di questo frammento, cui manca gran parte delle estremità inferiore e superiore, arriva ad otto centimetri e la larghezza ai quattro e mezzo. La forma apparisce lanceolato-bislunga, coi margini ampiamente sinuato-dentati e colle dentature molto distanti ed oltuse all'apice. Il nervo primario è piuttosto rilevato, e percorre la lacinia in forma flessuosa. I nervi secondarii, pure flessuosi, sono per lo più opposti o quasi opposti, ed escono obbliquamente dal primario, scorrendo equidistanti fino al margine della lacinia. Essi sono uniti trasversalmente ad angolo rello ed a distanze quasi eguali, da ramoscelli semplici lievemente flessuosi, che per tal modo danno origine a delle areole piuttosto grandi, di forma irregolarmente parallelogramma. Dai lati di queste si svolgono dei sottilissimi nervetti, che colla confluenza delle loro molteplici ramificazioni, formano una rete composta di maglie minute, più o meno regolarmente esagone.

Questa specie, proveniente dall' argilla ferruginosa del terreno giurassico medio di Matzdorf nella Slesia superiore, e figurata dal Göppert nell' Opera testè citata, fu poscia da lui brevemente descritta nella sua Memoria intitolata: Ueber die fossile Flort der mittleren Juraschichten in Oberschlesien, inserita negli Atti della Società di Storia Naturale della Slesia per l'anno 1845 alla pag. 143.

Un frammento dal quale non si può riconoscere la forma della fronda, ma che per la distribuzione dei nervi molto si avvicina a questa specie, fu da me rinvenuto nei monti di Marana, a settentrione di Crespadoro, nella Provincia Vicentina in una calcaria giallo-bruna che per la sua giacitura inferiore al Calcare ammonitico, deve riferirsi alla serie oolitica inferiore.

\section{Gen. XXIX. Sngevoptenes Presl.}

Frons stipitata simplex? vel digitata. Pinnae integerrimae 25-4, raro quinque. Nervi primarii excurrentes, vel saepius, ante apicem, evanescentes. Nervi secundarii obliqui, aequales, tenuis- 


\section{$-183$}

simi, iterato dichotomi, ramosissimi, ramulis omnibus reticulatim confluentibus, areolas plus minusve regulariter elongatorhomboideas vel hexagonas efficientibus. Fructificatio adhuc dubia.

Prest. in Stenxa. Vers. Fl. Vorw. II. pag. 164. - Görr. Gatt. foss. Pf. fasc. 5. 6. pag. 113.

- Brong. Tabl. Gen. Végét. foss. pag. 33.

Acrostichites. Görr. Syst. Filic. foss. pag. 284 (pro parte). - Ung. Gen. el Spec. Plant. foss. pag. 140 (pro parte).

\section{Sagenopteris cuneata Morris.}

Tav. XX. fig. 11.

S. fronde stipitata simplici obcordato-cuneata, apice profunde bilobata, vel pinnis duabus oppositis, obovatis, subemarginatis, basi cuneatis, sulpetiolatis, composita, stipite canaliculato; nervis aequalibus, tenuibus crebris, omnibus e basi flabellatim orientibus, pluries furcatis, ramulis areolato-reliculatis.

Sagenopteris cuneata. Monnis. Cal. Brit. foss. pag. 20. - Bunbunx. Foss. Pl. of Scarb. Quart. Joum. Geol. Soc, Lond. Vol. VII. pag. 184. - Zigxo. Enum. Filic, foss. form. Oolith. pag. 36.

Otopteris cuneala. Lindu. Hutr. Foss. Fl. Gr. Brit. II. pag. 203, tab. 155.

Adiantites irregularis. Görp. Syst. Filic. foss. pag. 385.

Cyclopteris cuneala. Prest, in Stensw. Vers. Fl. Vorw. II. pag. 135. - Urg. Gen. el Spec. Plant. foss. pag. 98.

In formatione oolithica ad Gristhorpe Bay prope Scarborough in Anglia; nec non in Alpibus Venetis in saxo calcareo lutescente seriei oolithicae inferioris ad montem Pernigotti in Provincia Veronensi.

La fronda è munita di uno stipite canaliculato del diametro di due millimetri, dall'estremità del quale sorgono due opposte foglioline o pinne, lunghe dai quindici ai dieciotto millimetri e larghe dai dieci ai dodici, di forma obovata un poco rotondata, coll' orlo irregolarmente emarginato e cuneate alla base ove si ristringono quasi in forma di picciuolo. Nanca in esse ogni traccia di nervo mediano, ma sono percorse da numerosi e sottili nervelti che tutti si diramano egualmente a ventaglio dalla base e 


\section{$-184$}

biforcandosi ripetutamente formano, colla congiunzione dei loro ramoscelli, una rete a maglie allungate che copre tutta la pagina della pinna.

Questa singolar forma trovata nei terreni oolitici d'Inghilterra presso Gristhorpe Bay non lungi da Scarborough fu collocata dagli autori nei generi Otopteris, Adiantites e Gyclopteris, perchè non posero attenzione alla reticolazione dei nervi così bene espressa nella figura che ne diedero i Signori Lindley e Hutton (Foss. Fl. Gr. Brit. II. tab. 155). 11 Prof. Morris fu il primo che la riferi al genere Sagenopteris (Gat. Brit. foss. pag. 20), ed il chiarissimo Sir C. Bunbury nella più volte citata sua Memoria sulle piante fossili di Scarborough inserita nel Vol. VII. del Quart. Journ. Soc. Geol. Lond. alla pagina 184, approvando l'operato del Prof. Morris, aggiunge di avere esaminato nella collezione del Sig. Bean, dei saggi in cui si scorge chiaramente la nervatura areolato-reticolata propria delle Sagenopteris, colla sola differenza cle non vi si osserva alcun indizio di nervo mediano. Egli accenna inoltre di avere veduto nella stessa collezione un saggio munito di una sola fogliolina obcordata, fessa nel terzo superiore e divisa in due lobi, che mostra la medesima distribuzione reticolata dei nervi. Queste diverse forme indussero quell'accurato osservatore a porre innanzi il dubbio che possano rappresentare le foglie seminali di qualche Felce e forse anco della Sagenopteris Phillipsii comune nello stesso terreno. Eguale dubbio sorgeva in me alla vista dell'esemplare trovato nella calcarea giallognola della formazione oolitica sul Monte Pernigotti nel Veronese, e del quale do qui la figura alla Tav. XX. fig. 11. Questo saggio presenta una fogliolina piuttosto crassa e parenchimatosa, di forma rotondata, obcordata, cuneata verso la base, fessa nel terzo superiore in due lobi ottusi e tutta percorsa da minutissimi nervetti uscenti dalla base in forma flabellata e più volte forcuti, coi ramoscelli che si anastomosano componendo una reticolazione a maglie assai minute ed allungate.

Non andrebbe forse troppo lontano dal vero chi supponesse che la piccola fronda semplicemente fessa all'apice, col crescere dell'età diventasse bipartita e poscia si dividesse in due foglioline staccate, che alla lor volta bipartendosi esse pure dessero origine ad una fronda digitata, fornita di quattro foglioline o pinne, come si osserva nella maggior parte delle Sagenopteris. 0ve tal progressione fosse vera, le forme testè descritte non sarebbero che lo stato giovane di queste pianticelle.

\section{Sagenopteris reniformis Zigno.}

Tav. XXI. fig. $6, \boldsymbol{a}, \boldsymbol{b}$

S. fronde stipitata, simplici, integerrima, reniformi undique marginem versus convexiuscula; nervis crebris tenuissimis subaequalibus, c basi fla- 
bellatim radiantibus usque ad marginem iterato furcatis, ramulis omnibus in areolas clongato-rhomboideas reticulatim confluentibus.

Sagenopleris renifornis. Zigno. Enum. Filic. foss. form. Oolith. pag. 35.

In saxo calcareo cinereo-fusco seriei oolithicae inferioris, ad Vallem Zuliani prope Roverè di Velo in Provincia Veronensi.

L'unico esemplare fin qui trovato lascia vederc breve porzione dello stipite, che si mostra segnato da numerose e finissime strie longitudinali interposte a delle minutissime coste. Sembra eziandio che fosse angoloso, poichè ove si congiunge alla base della fronda si scorge una piega trasversale, che forma una breve curva poligona. Lo stipite in quel punto misura due millimetri di larghezza. La fronda è reniforme coll'orlo interissimo e lievemente convessa tutt'all'intorno lungo il margine. $\mathbf{E}$ più larga che lunga, giungendo a ventiotto millimetri di larghezza, sopra una lunghezza di venti. Non è segnata da alcun nervo mediano, ed i nervetti che la percorrono sono tenuissimi, numerosi, quasi tutti eguali e sorgono dalla parte mediana della base irradiando a venlaglio verso il margine e biforcandosi ripetutamente fino all'orlo della fronda. Tutli i ramoscelli in cui dividonsi le forcature si anastomizzano fra di loro e formano una rete di areole romboidali allungate, simili a quelle delle altre specie di questo genere, ma un poco più piccole (Tav. XXI. fig. 6. b).

M'imbattei una sol volta in questa bella specie, che rinvenni con molti altri resti di Sagenopteris nel calcare grigio spellante alla serie oolitica inferiore, della Valle Zuliani presso Roverè di Velo nella Provincia Veronese.

La forma è diversa da quella di tutte le altre specie di questo genere, e rammenterebbe piuttosto le forme proprie del genere Cyclopteris, se la reticolazione delle nervature non ci additasse chiaramente il naturale suo posto fra le Sagenotteridi. Non intendo però escludere la probabilità che possa essere soltanto una fogliolina seminale di alcuna delle specie che giaciono negli stessi strati. Assai di spesso si trovano saggi di Sagenopteris muniti di una o due sole foglioline, e ciò potrebbe accennare ad un modo di svolgersi analogo a quello delle Marsilee indicato dal Fabre. E per vero i Professori Braun e Unger collocarono il genere Sagenopteris fra le Marsileacee, ma non avendo alcun autore seguita questa classificazione, lasciai per ora questo genere nella classe delle Felci. Sono però d'avviso che future scoperte ci obbligheranno a porre le Sagenopteris fra le Rizocarpee. 


\section{Sagenopteris angustifolia Zigno.}

Tav, XX. fig. 1. 2. 3. 4. 5, 6.7.8, 9. 10 .

5. fronde stipitata, digitata, stipite striato longitudinaliter bisulcato; pinnis $4-\mathbf{3}$, integerrimis, aequalibus, angustis, lanceolato-linearibus elongatis obtusis, basim versus altenuatis vel constrictis; nervis aequalibus, rectis, crassiusculis, pluries furcatis, ramulis anastomosantibus, areolas erectas, elongato-rhomboideas efficientibus.

Sagenopteris angustifolia. Zısro. Enum. Filic. foss. form. Oolith. pag. 35.

In calcareo lutescente seriei oolithicae inferioris ad montem Pernigotti prope S. Bortolamio in Provincia Veronensi.

Lo stipite, che raggiunge il diametro di due millimetri, è minutamente striato e percorso, lungo la superficie anteriore, da due solchi paralleli che corrispondono a due coste sporgenti nella parte dorsale. Le pinne o foglioline sono eguali fra di loro, angustissime, per lo più quattro, ma talvolta anche cinque, e mostrano di avere una consistenza piuttosto crassa. La loro forma è lanceolato-lineare allungata, coll'apice assai oltuso ed attenuate verso la base, ove nel punto d'inserzione sullo stipite si fanno ancor più ristrette. Misurano in lunghezza due centimetri ed hanno nel mezzo una larghezza di tre millimetri. Le figure 8. 9. della Tav. XX. rappresentano degli individui giovani in cui la lunghezza delle pinne non oltrepassa $\mathrm{i}$ dieci millimetri. I nervi che percorrono le pinne sono poco numerosi e tutti di eguale e piuttosto rilevante grossezza, avuto riguardo alla piccolezza delle pinne. Essi si biforcano ripetutamente, e, mercè la confluenza dei ramoscelli delle forcature, formano delle areole di forma molto stretta ed allungata, dirette verso l'estremità della pinna con andamento quasi parallelo al margine della medesina. Devo qui avvertire che nell'ingrandimento delineato alla fig. 10. della Tav. XX., la nervatura non è espressa colla dovuta precisione, scorgendovisi i nervi molto più sottili del vero, e troppo regolari e simmetriche le areole.

Alcuni corpicciuoli rotondi, colla superficie coperta di piccole asperità e talvolta muniti di lieve traccia d'un breve peduncolo, si trovano non di rado sparsi qua e la sulla roccia in vicinanza dei saggi di questa specie (Vedi Tav. XX. fig. 2.), e taluno ne osservai collocato presso all' estremitit inferiore dello stipite (Vedi Tav. XX. fig. 3.). Ritengo assai probabile che questi corpicciuoli possano avere una qualche attenenza colla fruttificazione, ancora ignota, di queste pianticelle, e ciò confermerebbe in qualche modo 


\section{$-187$}

la loro affinità colle Marsileacee: ma fino a che altri saggi non dieno maggior luce su questo argomento, sarebbe cosa prematura il pronunciare alcun giudizio.

Tutti gli esemplari di cui porgo la figura furono rinvenuti, nella calcaria giallognola a strati sottili che forma parte della serie oolitica inferiore, nel Monte Pernigotti, presso S. Bortolamio nella Provincia Veronese.

Questa specie si distingue da tutte le altre, non solo per la piccolezza delle sue dimensioni, ma principalmente per la diversa forma e ristrettezza delle foglioline, per la consistenza crassa delle medesime, per la grossezza e lo scarso numero dei nervi e per l'assoluta mancanza del nervo mediano. Tuttavia, anche riguardo a questa forma non sarebbe fuor di luogo il dubbio, che potesse essere per avventura lo stato giovane di qualche altra specie, e fors' anco della stessa Sagenopteris Phillipsii di Presl.

\section{Sagenopteris Phillipsii Presl.}

S. fronde longe stipitata, digitata; pinnis quaternis, integerrimis, lanceolato-ellipticis, vel late linearibus elongatis, basi apiceque attenuatis; nervo medio crasso excurrente, apice evanescente; nervis secundariis, tenuibus, aequalibus, obliquis, usque ad marginem iterato furcatis, ramulis omnibus in areolas hexagonoideas elongatas, reliculatim confluentibus.

Sagenopteris Phillipsii. Prese in Stenns, Vers. Fl. Vorw. II. pag. 165. - Monris. Cat. Brit. foss. pag. 19. - Zigro. Enum. Filic. foss. form. Oolith. pag. 35.

Glossopteris Phillipsii. Lixpl. Hutr. Foss. Fl. Gr. Brit. I. pag. 177, tab. 63, fig. 1-3 (exel. synon. Brovg.).

Acrostichites Phillipsii. Görr. Syst. Filic. foss. pag. 286. - Unger. Gen. et Spec. Plant. foss. pag. 141 .

Pecopteris paucifolia. Punc. Geol. Yorksh. I. pag. 119, tab. 8. fig. 8.

Gymnogramme Phillipsii. Exrıscsn. Farnkr, pag. 71.

In stratis arenaceo-argillosis inferioribus, formationis Oolithicae ad Gristhorpe prope Scarborough in Anglia.

La fronda è sorretta da un lungo stipite finissimamente striato il cui diametro misura dai due ai tre millimetri. Le pinne o foglioline che la compongono sono costantemente quattro, semplici e col margine intero, ora lanceolato-ellittiche, ora di forma am- 


\section{$-188$}

piamente lineare allungata, che si attenua tanto all'apice che verso la base. Variano d'assai in questa specie le dimensioni delle pinne. Poichè se ne trovano dei saggi in cui raggiungono una larghezza di quindici millimetri sopra cinque centimetri di lunghezza; in altri le pinne sono larghe solo dieci millimetri quantunque giungano ad una lunghezza di otto centimetri, ed uno ne conservo nel mio Gabinetto in cui le pinne non superano i due centimetri in lunghezza ed i quattro millimetri in larghezza. Il nervo mediano è piuttosto appariscente e scorre ben marcato fin presso all' apice della pinna gradatamente assoltigliandosi ed ivi svanendo. I nervetti secondarii sono assai numerosi e sottili e si biforcano ripetutamente volgendosi obbliquamente verso i margini delle pinne, formando, colla congiunzione delle loro ramificazioni, una rete a maglie esagone molto allungate.

Questa specie si rinviene negli strati arenaceo-argillosi della serie oolitica inferiore a Gristhorpe presso Scarborough nell' Inghilterra.

Una delle forme in cui le pinne si mostrano alquanto più larghle, fu distinta dal Chiarissimo Sig. Bean col nome di Glossopteris Dumnii (Vedi Buns. Mem. on foss. Pl. of Scarb. in Quart. Journ. Geol. Soc. Lond. Vol. VII. pag. 186); ma le variazioni che vedemmo essere frequenti nei saggi della presente specie, non permettono di accogliere una separazione fondata sopra questo solo carattere.

\section{Sagenopteris Goeppertiana Zigno.}

Tav. XXI. fig. 1. a, b, fig. 2. 3. 4. 5. Tav. XXII. fig. 1. 2.

S. Pronde stipitata, digitata, stipite crasso striato; pinnis 4, inaequalibus, integerrimis, subpetiolatis, mediis latis, obovato-cuneatis apice rotundatis, lateralibus lanceolato-oblongis, obtusis, inaequilateris; nervo medio crasso canaliculato, longe ante apicem, evanescente; nervulis obliquis, tenuibus, usque ad marginem iterato-furcatis, ramulis omnibus in areolas hexagonoideas elongatas, reticulatim confluentibus.

Sagenopteris Goeppertiana. Zigno. Enum. Filic. foss, form. Oolilh. pag. 36.

Sagenopteris rolundala. Zigko. Loc. cit. pag. 35.

Sagenopleris Brauniana. Zigro. Loc. cit. pag. 36.

Sagenopteris Brongniartiana. Zıкso. Loc. cit. pag. 36. 


\section{$-189-$}

In saxo calcareo cinereo seriei oolithicae inferioris ad Vallem Zuliani prope Roverè di Velo in Provincia Veronensi.

Lo stipite in questa specie è piuttosto robusto e raggiunge negli esemplari più grandi il diametro di quattro millimetri. Esso è percorso da numerose strie longitudinali e nella sua estremità superiore si parte in quattro brevissimi picciuoli che vanno a formare il nervo mediano delle pinne. Quest' ultime sono quattro, ben disgiunte fra

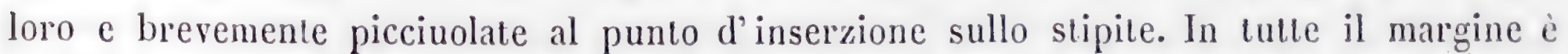
intero, ma non sono di egual forma e dimensione. Le medie (Tav. XXI. fig. 4. e Tav. XXII. fig. 2) sono ampiamente obovate, rotondate nell' estremità e cuneate verso la base, e misurano per lo più dai nove ai dieci centimetri in lunghezza. La loro larghezza presa nel terzo superiore supera i quattro centimetri. Le pinne laterali hanno invece una forma lanceolato bislunga coll'apice semplicemente outuso e coi lati ineguali, l'esterno essendo più largo e l'interno più stretto, ed attenuato verso la base (Tav. XXI. figg. 1. Tav. XX. fig. 1.). L'ordinaria larghezza di queste pinne laterali è di tre centimetri e la lunghezza sta fra i sette e gli olto centimetri. Il nervo mediano che si prolunga dal picciuolo è al basso piuttosto largo e marcato, ed ha una depressione nel mezzo che per breve tratto lo fa apparire scanalato. All'incirca alla metà della pinna, e quindi molto prima di giungere all' apice, esso svanisce risolvendosi in numerosi e soltili nervetti secondarii eguali a quelli che sorgono obbliquamente da' suoi lati. Tutli i nervetti secondarii si dirigono a ventaglio verso l'orlo delle pinne, biforcandosi ripetutamente in tulta la loro estensione, e $\mathrm{i}$ ramoscelli risultanti dalle forcature si anastomizzano fra di loro, e danno origine ad una rete di maglie più o meno regolarmente esagone e molto allungate (Vedi Tav. XXI. fig. 1.b).

Gli esemplari di questa specie sono abbondantissimi nella calcaria grrigia, spettante alla serie oolitica inferiore, della Valle Zuliani presso Boverè di Velo nella Provincia Veronese.

L'esame di un buon numero di saggi, ni pose in grado di seguire i passaggi delle varie forme che distinsi come specie diverse nella mia, Enumeratio Filicum fossilium formationis oolithicae pag. 35-36., alla forma adulta, che descrissi nello stesso lavoro, sotto il nome di Sagenopteris Goeppertiana, e di cui mi fu dato rinvenire, dopo quell'epoca, degli esemplari migliori (Vedi Tav. XXI. fig. 1. $a$, b, fig. 4. e Tav. XXII. fig. 1. 2.).

La Sagenopteris Brauniana, Tav. XXI. fig. 2., è la medesima specie allo stato giovane, e la Sagenopteris Brongniartiana, Tav. XXI. fig. 3., una forma intermedia non ancor giunta al suo pieno accrescimento. In quanto alla Sagenopteris rotundata, Tav. XXI. 
fig. 5., essa non è che una delle pinne medie della Sagenopteris Goepperliana, alquanto corrosa nel margine.

L'unica specie che a prima giunta paleserebbe una qualche rassomiglianza colla presente, si è la Sagenopteris Rhoifolia di Presl, specialmente dopo che il chiarissimo Prof. Schenk (Foss. Fl. d. Grenssch. d. Keup. u. Lias Frank, pag. 58) notava di quest'ultima le frequenti variazioni (probabilmente dovute a differenza di età), e ad essa riuniva parecchie forme descritte come specie diverse da Presl, da Göppert, da Unger e da altri. Però la Sagenopteris Goeppertiana, esaminata nei varii suoi stadj, si distingue non solo per aver sempre tutte le pinne molto più ottuse, ma perchè negli esemplari adulti le pinne medie si mostrano costantemente coll'estremità più ampia e rotondata (Tav. XXI. fig. 4. 5., Tav. XXII. fig. 1. 2.). Oltre a ciò il nervo mediano, che nella $S(\ell-$ genopteris Rhoifolia di Presl è rilevato e percorre le pinne fin quasi all'apice, nella nostra specie invece è scanalato alla base e svanisce circa alla metà delle pinne, risolvendosi in nervetti reticolati eguali a quelli (Tav. XXI. fig. 1. $a, 1 . b$ ) che inferiormente si diramano da' suoi lati.

\section{Gen. XL. Crevis Lindl. Hutt.}

Frons profunde pinnatifida, rachide crassa striato-sulcata. Laciniae approximatae, lineares, elongatae, integerrimae, basi dilatatae, sinu acuto interstinctae. Nervi aequales, crassiusculi, e rachide orientes, basim versus divergentes, dein approximati, paralleli, excurrentes, furcati, ramulis in areolas oblique elongato-rhomboideas confluentibus.

Livde. Hutt. Foss. Fl. Gr. Brit. II. pag. 63. - Stensв. Vers. Fl. Vorw. II. pag. 162. Brong. Tabl. Gen. Végét. foss. pag. 62. - Usa. Gen. et Spec. Pl. foss. pag. 307.

\section{Ctenis falcata Lindl. Hutt.}

Tav. XXIV. fig. 1, 2. 3.

C. fronde profundissime pinnatifida, rachide aequali, recta, multistriata, :3-millin. circiter crassa; laciniis suboppositis, linearibus, integerrimis, elongatis, subarcuatis patulis, leviterque falcatis, basi paululum dilatatis ac decurrentibus, sinu acuto interstinctis, terminali lanceolata, basi angustala; 
nervis crebris parallelis, basim versus divergentibus, huc illuc furcalis, ramulis in areolas irregulares, oblique elongato-rhomboideas, confluentibus.

Clenis falcala. Linde. IIutr. Foss. Fl. Gr. Brit. II. pag. 63, lab. 103 . - Stenмi. Vers. Fl. Vorw. II. pag. 162. - Urg. Gen. el Spec. Pl. foss. pag. 307. - Monns. Cat. Brit. foss. pag. 6.

Cycadites sulcicaulis. Pmil. Geol. Yorksh. I. pag. 119, tab. VII. fig. 21, a, b.

In formatione oolithica ad Gristhorpe prope Scarborough in Anglia frequenter orcurrit.

Le frondi di questa Felce dovono essere state piuttosto grandi, poichè se ne trovano dei frammenti che hanno una larghezza di oltre undici centimetri sopra diciassette di lunghezza, i quali sono ancora ben lontani dal poterci dare una idea esatta della sua dimensione, mancando essi della base e dell'apice, nonchè di tutte le estremità delle lacinie, mentre, per la grossezza della rachide, e la larghezza delle lacinie al punto ove rimasero troncate, evvi ogni ragione di credere che si prolungassero ancora non poco.

La fronda è profondamente pinnatifida e sorretta da una rachide, avente un diametro di cinque millimetri all'incirca, segnata per lo lungo da minutissimi solchi o strie, alternanti con delle sottili costicine, il cui andamento è simile a quello dei nervi che percorrono le lacinie. Le lacinie sono lineari allungate, un po' arcuate e leggermente falcate, col margine intero e molto avvicinate presso la base, ove allargandosi, confluiscono e formano ala alla rachide, disgiunte da un seno alquanto acuto e più o meno anģusto. La lacinia terminale della fronda è di forma lanceolata, attenuata verso la base ove si congiunge all' apice della rachide. La larghezza delle lacinie varia dai cinque ai dieci millimetri, ma nulla può dirsi circa alla loro lunghezza, imperciocchè in tutte le figure che esistono pubblicate ed in tutti gli esemplari che ho esaminato mancano le estremità delle lacinie.

I nervetti da cui sono percorse nascono dalla rachide piuttosto discosti l'uno dall'altro, e poscia si avvicinano, per cui nel senso inverso alla lor direzione appariscono divergenti e rivolti parte verso il lato superiore della lacinia e parte verso il lato inferiore; proseguono poi paralleli e ben marcati per tutta la lunghezza della lacinia, facendosi però di tratto in tratto forcuti, e mettendo dei ramoscelli più sottili ed obbliqui cle si congiungono fra di loro e formano colla loro confluenza delle areole irregolarmente romboidali molto allungate (Vedi Tav. XXIV. 1., a.). 


\section{$-192$}

Questa specie è posta dal Sigg. Leckenby nel novero di quelle che si trovano soltanto nell'argilla calcarifera scissile della serie oolitica inferiore a Gristhorpe presso Scarborough nell' Inghilterra.

Varj furono i pareri degli Autori sulla Classe o famiglia cui spettar potesse questa curiosa forma. Il Prof. Phillips la collocò fra le Cicadee. I Signori Lindley e IIutton opinarono dovesse prender posto fra le Palme. Sternbergg la annoveró fra le Felci. Unger invece la pose fra le sue Cicadeacee dubie, e Brongniart nel suo Tableau des Genres de Végétaux fossiles, descrisse questo genere ponendolo nelle Cicadee subito dopo il genere Zamites.

Il Prof. Braun ritenne egli pure il genere Gtenis fra le Cicadeacee, e vi riuni alcune forme da lui scoperte nel lias di Bayreuth (Ctenis angusta; C. abbreviata; G. marginata; G. inconstans. Braun in Münst. Beitr. zur Petref. VI. Heft. Ueber foss. Zamien. etc. pag. 34-42), che a dir vero non hanno alcuna analogia colla specie di Gristhorpe e sono fornite di nervetti semplici, e privi di qualsiasi traccia di ramoscelli secondarj reticolati. Le forme descritte dal Braun quali specie appartenenti al genere Ctenis, sono realmente da annoverarsi fra le Cicadeacee nel genere Pterophyllum, mentre la Ctenis falcata dei Signori Lindley e IIutton si collega evidentemente alle Felci per la reticolazione prodotta dalla confluenza dei ramoscelli uscenti dalle forcature dei nervi.

Come feci pel Diclyophyllum rugosum Lind. Hutr., di cui pubblicai la figura di un esemplare da me posseduto, perchè in esso era chiaramente visibile la distribuzione dei nervi, mentre in nessuna delle figure esistenti era stata esaltamente espressa; cosi stimai utile del pari, il porgere le figure di tre esemplari della Gtenis falcata Lrnot. Iutr., pure appartenenti alla mia collezione, sui quali si scorge bene distinta la relicolazione dei nervetti, che forma l'argomento principale per cui fui indotto ad escluderla dalle Cicadeacee e ad annoverarla fra le Felci.

\section{Opdo XIV. Gleichentaceae.}

\section{Gen. XLI. GLichevites Göpp.}

Frons dichotoma pinnata vel bipinnata. Pinnae pluries dichotomae, ramis sparsis divaricatis, pinnulis parvis, coriaceis. Nervi flabellato-pinnati. Sori rotundi.

Görp. Syst. Filic. fuss. pag. 172 el pag. 181. - Uxger. Gen. et Spec. Plant. foss. pag. 207. 


\section{Gleichenites elegans Zigno.}

Tav. X. fig. 1. 1, a. fig. 2.

G. fronde irregulariter pinnato-dichotoma, rachide striata crassiuscula, hasi stricta, superne flexuosa; pinnis angustis, pinnatisectis, gracilibus, remolis, imis oppositis, superioribus alternis, omnibus pluries dichotomis, ramulis angulo recto vel obtuso divergentibus, divaricatis, sparsis; segmentis brevissimis, oblique semirotundis, alternis vel suboppositis, tota base rachi adnatis, sinu angusto discrelis; nervo primario basi excentrico in nervulos furcatos, tenuissimos, vix conspicuos, resoluto.

Gleichenites elegans. Zıgno, Enum. Filic. foss. form. Oolith. pag. 37.

In calcarea cinerea seriei oolithicae inferioris ad Vallem Zuliani prope Roverè di Velo in Provincia Veronensi.

La fronda di questa bella specie passa dalla forma irregolarmente pinnata alla piì volte dicotoma, ed è munita di una rachide consistente e finamente striata, il cui diametro non oltrepassa i due millimetri, e che corre ritta per alcun tratto e poscia procede flessuosa fino all'estremità. Le pinne inferiori sono opposte e distanti fra di loro circa quattro centimetri. Le superiori sono alterne e gradatamente più avvicinate si che giungono ad essere discoste appena un centimetro presso l'apice della fronda. La loro larghezza non supera i tre millimetri e si dicotomizzano ripetutamente, fin dalla base, suddividendosi ad angolo retto, oppure oltuso, in rami sparsi ed assai divaricati.

Le pinne sono in tutta la loro estensione frastagliate fittamente in tanti segmenti un poco obliqui, semirotondi e brevissimi, non giungendo che ad un millimetro e mezzo di lunghezza sopra una larghezza di due. Quantunque assai avvicinati, sono però disgiunti da un seno angusto nel punto ove con tutta la base s'inseriscono sulla rachide, mostrandosi per lo più alterni ed in qualche parte della fronda anche quasi opposti. Alla base dei segmenti, ma fuori di centro, scorgesi un nervo primario brevissimo che tosto si divide in pochi nervetti secondarii forcuti appena visibili.

Sebbene non abbia ancor potuto raccogliere questa specie colla fruttificazione, tuttavia credo di non iscostarmi dal vero ponendola nel genere Gleichenites. L'aspello generale della fronda, le ripetute dicotomie delle pinne, la forma e piccolezza dei segmenti la avvicinano in modo non dubbio alle Gleichenie della Flora vivente. A dir vero 


\section{$-194$}

il genere Gleichenites di Göppert non fu ammesso dal Brongniart (Vedi Tabl. Gen. Végét. foss. pag. 15), perchè basato sul solo carattere della biforcazione della fronda, ma nel caso presente, la forma che io vi riferisco ci porge caratteri sufficienti per meglio circoscrivere lo stesso genere.

Ne trovai da prima non pochi frammenti, che per lungo tempo mi lasciarono incerto sul genere cui potesse appartenere. Il bell' esemplare completo ('Tav. X. fig. 1.) rinvenuto nella roccia calcaria grigia della serie oolitica inferiore, nella Valle Zuliani presso Roverè di Velo nel Veronese, venne a porre in chiaro le analogie che collegano questa forma alle Gleichenie dell'epoca attuale.

\section{Gleichenites? Desnoyersii Zigno.}

G. fronde... pinnis pinnaliseclis elongatis, latiusculis, rachide crassa; segmentis oppositis, approximatis, semirotundis, oblusis, usque ad basim discretis, rachi adnatis et perpendicularibus; nervis nervulisque nullis distinctis.

Gleichenites? Desnoyersii. Zigno. Enum. Filic. foss. form. Oolith. pag. 37.

Pecopteris Desnoyersii. Brong. Ann. Sc. Nat. IV. pag. 421, tab. 19, fig. 1.; Prodrom. pag. 59 ; Hist. Végét. foss. I. pag. 366, tab. 129, fig. 1. - Unger. Gen. et Spec. Plant. foss. pag. 178.

Filicites Desnoyersii. Presl, in Sternis. Vers. Fl. Vorve. 11. pag. 174.

In stratis formationis oolithicae ad Hamers in Gallia.

Di questa forma non si conosce che il frammento di pinna trovato nell'oolite a Mamers in Francia ed illustrato dal Brongniart. Esso è lungo all' incirca quattro centimetri e mezzo, e largo appena dieci millimetri ed ha le estremità troncate, senza che, verso le medesime, si scorga alcuna diminuzione nel diametro, per cui è a ritenersi che abbia appartenuto ad una pinna piuttosto allungata. La rachide che lo percorre è liscia, dritta e rilevata, e misura in diametro poco più di un millimetro. La pinna è divisa fino alla rachide in segmenti perfettamente opposti, semirotondi, otlusissimi che sembrano avere il margine leggermente incurvato verso la pagina inferiore. Sono inseriti con tutta la base perpendicolarmente sulla rachide e disgiunti fra di loro, quantunque al punto d'inserzione appariscano contigui. La loro lunghezza non oltrepassa i quattro millimetri ed alla base misurano dai quattro ai cinque millimetri di larghezza. Della nervatura non si può scorgere alcuna traccia. 
Questa forma non so per qual ragione collocata dagli autori nel genere Pecopteris non ha alcun carattere che giustifichi la sua spettanza a quel genere, e palesa invece maggiori analogie col genere Gleichenites. La forma dei segmenti rammenta quella dei segmenti della Gleichenites elegans Zigro, ma nella specie presente sono molto più grandi, più decisamente opposti, affatto perpendicolari alla rachide, e cosi avvicinati che verso la base si toccano.

\section{Gen. X Lit. Laccoptenis Presl.}

Frons stipitata digitato-pinnatifida. Pinnae pinnatilidae vel pinnatisectae. Nervi primarii excur!entes. Nervi secundarii dichotomi, ramulis furcatis simplicibusve, mediis in medio dorso soriferis. Sori rotundi biseriales, fovea semiglobosa immersi. Sporangia in orbem disposita, annulo multiarticulato instructa. Sporae teträedricae.

Prest in Sternas, Vers. Fl. Vorw. 11. pag. 115. - Göpr. Gall. foss. PR. fasc. 1. 2. pag. 7. - Brong. Tabl. Gen. Végét. foss. pag. 29. - Unger. Gen. et Spec. Plant. foss, pag. 205. Schenti. Foss. Fl. d. Grenzsch. d. Keup. u. Lius Frankens. pag. 93.

\section{Laccopteris Phillipsii Zigno.}

I. fronde longe stipitata, digitato-pinnatifida, stipite crasso, striato, superne dilatalo; pinnis linearibus elongalis, profundissime pinnatifidis, basim versus allenuatis; laciniis suboppositis patulis, linearibus, integerrimis, subfalcatis, incurvis, apice oblusis, basi confluentibus, sinu rotundo discrelis, infimis brevissimis; nervis secundariis e nervo medio excurrente, angulo subrecto egredientibus, dichotomis; soris biserialibus rotundis, prominulis, approximatis, puncto centrali depresso instructis; sporangiis in orbem dispositis.

Laccopteris Phillipsii. Zacro. Enum. Filic. foss. form. Oolith. pag. 37.

Pecopteris caespitosa. Puncups. Geol. Yorksh. I. pag. 119, tab. 8. fig. 10. - Monns. Cat. Brit. foss. pag. 15. - Bunbuny. Foss. Pl. of Scarb. in Quart. Journ. Suc. Geol. Lond. Vol. VII. pag. 186.

Pecopteris polydactyla. Lecrexbr. Oolit. Pl. of Scarb. in Quart. Jaurn. Soc. Geol. Lonl. Vol. XX. pag. 80 (excl. synon.) tab. 11, fig. 1, $a, b$. 


\section{- 196}

In formatione oolithica ad Cloughton prope Scarborough in Anglia.

Lo stipite si mostra longitudinalmente striato e piuttosto robusto. Esso ha un diametro di quattro millimetri, ma nell' estremità superiore si espande giungendo ad una larghezza di sei, ed ivi si divide in forma digitata dando origine a nove pinne lineari assai allungate e profondamente pinnatifide, la cui lunghezza oltrepassa talvolta i tredici centimetri, mentre la larghezza varia dai dieci ai quattordici millimetri. Però procedendo verso la base esse vanno lentamente altenuandosi fino a che si riducono a misurare due soli millimetri e mezzo, presso il punto ove si congiungono allo stipite. Le lacinie in cui sono frastagliate le pinne, sono per lo più opposte e patenti, col margine intero, di forma lineare, curvate all'insù e quasi falcate, coll'apice ottuso, e confluenti alla base presso il punto d'inserzione ove sono separate da un seno rotondo piu o meno angusto. La loro maggiore lunghezza arriva agli olto millimetri e la larghezza appena a due. Diminuiscono poi gradatamente nelle dimensioni procedendo verso la base delle pinne, ove, tanto in larghezza che in lunghezza, non passano il millimetro. Un sottile ma ben rilevato nervo mediano le percorre fino all'apice, e dai lati di quello sorgono ad angolo quasi retto i nervetti secondarj, poco appariscenti ma che sembrano dividersi in forma dicotoma. La fruttificazione è collocata lungo i lati del nervo mediano negli spazj interposti ai nervetti secondarj, cosi formando due serie piuttosto fitte di sori rotondi e sporgenti, che per la depressione del loro centro presentano la forma di una ciambella, e lasciano quindi arguire, che gli sporangi fossero disposti a cerchio, intorno ad un punto centrale.

Questa specie si trova ora con qualche frequenza nei depositi fitolitiferi dell'oolite a Cloughton presso Scarborough nell' Inghilterra.

Sir C. J. Fox Bunbury nella citata sua Memoria sulle piante fossili di Scarborough, accennò pel primo alla rassomiglianza esistente fra questa forma e quelle comprese nei generi Laccopteris di Presl e Andriania di Braun, e l'accurato esame di alcuni esemplari muniti della fruttificazione, mi persuase della convenienza di collocarla appunto nel genere Laccopteris.

La forma e la distribuzione digitata delle pinne, la disposizione dei nervi che percorrono le lacinie, quella dei sori e la loro figura rotonda, circolare e sporgente, prodotta dall' ordinamento degli sporangi intorno ad un punto centrale depresso, mi sembrano giustificare abbastanza questo trasferimento. Siccome poi il nome specifico di $P e$ copteris caespitosa datole da Phillips, esprimeva l'idea che le pinne uscissero da una specie di cespo, mentre fu riconosciuto di poi che si diramavano in forma digitata dal- 


\section{$-197-$}

l'estremità espansa di uno stipite alquanto prolungato, cosi stimai opportuno di non conservare un nome alludente ad un carattere che non esisteva, e vi ho sostituito invece quello del primo autore che ne diede la figura.

Il Sig. Leckenby riunisce questa forma alla Pecopteris polydactyla di Göppert, che Ettingshausen annovera fra i sinonimi della sua Alethopteris Goepperti, unitamente alla Alethopteris elegans Görp., alla Pecopoteris Comybearii Duns., ed alla Pecopteris Althansii dello stesso Dunien (Vedi Etringsur. Flora d. Wealden periode pag. 16).

Non è qui il luogo d'investigare se tutte queste forme appartengano a quella sola specie che l'Ettingshausen distinse col nome di Alethopteris Goeppertii; ci basterà soltanto esaminare se alcuna di esse corrisponda a quella che abbiamo descritta e di cui possediamo dei saggi ben conservati. Ora, è forza riconoscere che nessuna di queste forme ha le lacinie delle pinne confluenti alla base, chè anzi le hanno ivi perfettamente distaccate fra di loro, in guisa che le pinne risultano pinnate, piuttosto che pinnatifide. Oltre a ciò, nell'Alethopteris Goepperti di Ettingshausen e nella Pecopteris polydactyla di Göppert, le pinnule, invece di essere ottuse, sono alquanto acuminate all'apice. Egualmente discoste fra loro fin dalla base sono le pinnule della Pecopteris Alhausii Duxw. della Pecopteris Comybearii Duкn. e della Alethopteris elegans Göpr. e per sopra più le due ultime hanno le pinnule alterne, mentre nella Laccopteris Phillipsii sono per la maggior parte opposte. Queste differenze, avvalorate anche dall'appartenenza di tutte le sopra citate specie ad una formazione assai più recente, mi determinarono ad escluderle dalla sinonimia della specie di cui qui si tratta.

\section{Laccopteris Botzana Zigno.}

Tav. XXII. fig. 3. a, b, c.

L. fronde stipitata, digitato-pinnatifida; pinnis elongato-lanceolatis, obtusis, pinnatisectis; laciniis alternis approximatis, contiguis, late-linearibus, integerrimis, apice rolundatis, basi tola latitudine oblique insertis, sinu acutissimo interstinclis, superioribus decrescentibus, infimis brevioribus obovatis, basi allenuatis, decurrentibus; nervo medio flexuoso, apice in nervulos furcatos resoluto; nervis secundariis oblique egredientibus, furcalis, arcuatis, tenuissimis, sorigeris crassioribus; soris biserialibus, remotiusculis, semi-immersis.

laccopteris Rolzana. Zusa. Enum. Filic foss, form. Oulith. pag. 38 


\section{$-198-$}

In stratis calcareae cinereae, seriei oolithica inferioris, prope Rotzo in Provincia Ficentina, una tantum vice mihi occurrit.

La fronda è sorrelta da uno stipite lievemente striato, che ha un diametro d'un millimetro e mezzo, dalla cui estremità superiore si diramano in forma digitata cinque o sei pinne lanceolato-allungate profondamente pinnatifide, ottuse all'apice ed attenuate verso la base. Queste pinne, che hanno una rachide consistente e striata, sono lunghe tre centimetri e mezzo e larghe appena un centimetro, e si dividono in lacinie alterne, inserite obbliquamente con tutta la base sulla rachide, di forma largamente lineare, col margine intero e l'estremità rotondata, molto avvicinate e disgiunte da un seno assai acuto, lunghe cinque millimetri e larghe due e mezzo.

Verso le due estremila della pinna decresce gradatamente la lunghezza delle lacinie e le inferiori sono brevissime, più distaccate fra loro, di forma obovata, allenuate verso la base e decorrenti (Tav. XXII. fig. 3. b). Il nervo mediano delle lacinie scorre dapprima flessuoso, indi si risolve verso l'apice in nervetti forcuti. I nervetti secondarii che escono da quello obbliquamente si biforcano arcuandosi verso i margini delle lacinie. Questi nervetti sono in generale assai tenui, ma fra mezzo a loro se ne osservano alcuni assai più grossi degli altri i quali portano la fruttificazione (Tav. XXII. fig. 3. c). Questa si compone di sori rotondi mezzo immersi in una piccola cavità, posti in serie laterali al nervo mediano e piuttosto distanti l'uno dall'altro.

Questa specie fu da me trovata una sol volta nella calcaria grigia spettante alla serie oolitica inferiore dei contorni di Rotzo nei Sette Comuni della Provincia di Vicenza.

Quantunque non mi sia stato possibile il riconoscere la struttura e disposizione degli sporangi, tultavia la forma digitata della fronda, il modo di frastagliarsi delle pinne, la distribuzione dei nervi secondarj delle lacinie, fra i quali alcuni più grossi sorreggono i sori che sono in parte immersi in una piccola cavità, mi sembrano porgere caratteri sufficienti a giustificare il collocamento di questa forma nel genere Laccopteris. La specie poi si distingue da tulte le altre di questo genere, principalmente per avere le lacinie delle pinne, di forma largamente lineare e contigue fin presso all'apice, ove terminano rotondandosi senza prima attenuarsi. 


\section{Ordo XV. Marattiaceie.}

\section{Gen. LET. Thevionemis Brong.}

Frons petiolata, simplex vel pinnata. Nervus primarius rigidus excurrens. Nervi secundarii tenues, angulo recto vel acuto egredientes, paralleli, 1-2-5-furcati, interdum simplices. Sori lineares oblongi, prominentes ad utrumque nervulorum latus, marginem versus, collocati. Sporangia libera, numerosa, prominula, in quovis soro transversim biserialia. Sporae oblongae.

Bnorg. Prodrom. pag. 61; Hist. Végét. foss. I. pag. 262; Tabl. Gen. Végét. foss. pag. 21. Stenxв. Vers. Fl. Vorw. II. pag. 138 (ex parte). - Görr. Gen. Pl. foss. Lief. 3.-4. pag. 51. Ung. Gen. et Spec. Pl. foss. pag. 211 (ex parte). - Scuexr. Foss. Fl. Keup. Lias Frank. pag. 99.

Aspidites. Görr, Syst. Filic. foss. pag. 349 (ex parte).

Le forme comprese finora dagli autori in questo genere si collocarono ora fra le Marattiacee, ed ora fra le Daneacee, ed una parte di esse fu dal Bornemann annoverata fra le Cicadeacee nel genere, cui, per errore, diede il nome di Strangeriles, invece di Stangerites come avrebbe dovuto appellarsi, perchè fondato sopra apparenti analogie colla Stangeria paradoxa di Moore (Bonnen. Org. reste d. Lettenk. Thüring. pag. 59).

Il Chiariss. Prof. Göppert che nel suo Systema Filicum fossilium stampato nel 1836 aveva posto le Teniotteridi nel suo genere Aspidites, avendo poscia scoperto la fruttificazione della Taeniopteris Mïnsterii, che descrisse e figurò nell'Opera intitolata: Les Genres des Plantes fossiles che vide la luce nel 1841, le ricollocò nuovamente nel genere Taeniopteris, ponendo quest'ultimo fra le Daneacee, nel che fu poi seguilo dall'Unger. Ma recentemente il Chiarissimo Prof. Schenk illustrò siffattamente la fruttificazione della Taeniopteris Münsterii Görp., porgendo cosi esatte figure dei sori, degli sporangi e delle spore, da riconoscervi una somma analogia colla frutlificazione delle Angiopteris della Flora altuale (Vedi Scnexк, Foss. Fl. Keup. u. Lias Frank. pag. 99, Tav. XX. fig. 2.-8.), e questa analogia cosi marcata induceva lo Schenk a porre fra le Maralliacee le varie specie di Taeniopteris che descrisse nella sua Opera, non senza ricordare come il Chiarissimo Prof. (). Heer avesse scoperto nel Keuper della Svizzera un esemplare della Taeniopteris marantacea. Sterab. fornito di una fruttificazione assai 
diversa da quella della Taeniopteris Münsterii Görr., ed invece cosi somigliante a quella delle Danee della Flora vivente del Brasile, che lo stesso Prof. Ileer consigliava separarla dal genere Taeniopteris e riporla fra le Daneacee col nome di Danaeopsis marantacea (IEER, Urw. d. Schweiz. pag. 54).

Queste distinzioni fondate sulla diversa forma e distribuzione della frultificazione, sono di tale importanza da rendere indispensabile di seguire il parere del Prof. Ileer dividendo le Teniotteridi in due gruppi generici, l'uno destinato ad accogliere quelle specie che hanno la fruttificazione somigliante a quella delle Marattiacee, l'altro quelle che colla loro fruttificazione si mostrano analoghe alle Daneacee. Il Prof. Ileer consiglia pur anco di serbare il nome di Taeniopteris pel primo, e propone il nome generico di Danaeopsis pel secondo.

Abbracciando interamente l'opinione del Prof. Ileer, confermata anche dalle recenti osservazioni del Prof. Schenk, io conservo adunque il genere Taeniopteris circoscritto secondo i sopra esposti criterii, e ben volentieri adotterei il nome di Danueopsis per le altre forme che devono prender posto fra le Daneacee, se non vi ostasse la circostanza, che fino dal 1845, un tal nome veniva adoperato dal Presl per la Danaea paleacea del Raddi, avendola egli separata dalle altre specie di questo genere sotto la denominazione di Danaeopsis paleacea (Vedi PresL, Suppl. Tentam. Pteridographiae pag. 39). Imperciocchè è ben noto come per denominare una forma fossile, collo stesso nome generico impiegato per un genere della Flora vivente, sia d'uopo ch'esista fra di loro una perfetta identita di caratteri generici, il che nel caso presente non si riscontra, poichè della Danaeopsis di Presl non si conosce la frutlificazione, e questo genere fu creato da Presl sopra una semplice pinna sterile della Danaea paleacea di Raddi, Monographia Filic. Brasil. pag. 76. tav. 5. fig. 2, in cui la reticolazione dei nervi fu il solo carattere che indusse il Presl a separarla dal genere Danaea di Smith, e questo carattere manca affatto nelle forme fossili che pel modo di fruttificazione si associano alle Daneacee.

Anche il genere Danaeites di Göppert, System. Filic. foss. pag. 172, 380, fondato su quella forma cui prima aveva dato il nome di Asplenites Damaeoides, non presenta, nell'unica specie che vi è compresa, caralteri sufficienti per istabilire la sua affinità colle Danee. Però questo genere fu conservato dall' Unger e poscia dall'Ettingshausen che vi riferi la sua Daneites Schlotheimi, trovata nel terreno cretaceo d'Aquisgrana. Ed è forza convenire che l'esemplare descritto e figurato dall'Ettingshausen s'assomiglia assai più alle pinne della Danaea nodosa Smitr, in confronto di quello che servi al Göppert per istabilire il genere Danaeites (Etringsn, Die Acrobr. d. Kreidegeb. v. Aachen u. Maestricht, Denks. d. K. Acad. d. Wissensch. Wien. pag. 202, tav. III. figg. 1.). Perciò stimai opportuno di serbare questo genere, ampliandone la diagnosi colla 
giunta di que' caratteri che ci furono segnalati dalla scoperta del Prof. Heer, evitando in tal guisa la introduzione di un nuovo nome generico per comprendervi le Taeniopteris fornite di una fruttificazione analoga a quella delle Danee.

Siccome poi fra le Teniotteridi finor conosciute non si rinvenne la fruttificazione bene spiegata che sopra due sole specie, la Taeniopteris Münsterii Göpr. e la Taeniopteris marantacea Sternb., cadauna delle quali, come vedemmo, serve di tipo per uno dei due generi in cui è d'uopo dividere il genere Taeniopteris, cosi rimane ancora assai malagevole ed incerta la ripartizione in questi due gruppi generici di tutte le altre specie, di cui ci è tuttora ignota la fruttificazione. Limitandomi nella presente opera alle sole specie dell'oolite, nelle quali a dir vero non ne trovai alcuna che lasciasse scorgere indizj precisi della fruttificazione, ho dovuto ricorrere alla investigazione di tutti quegli altri caratteri, il cui complesso mi ha sembrato porgere dati sufficienti per fissare le basi di quelle analogie che mi parvero acconcie a giustificare la loro distribuzione nei due generi Taeniopteris e Danaeites.

Na l'argomento principale che m'indusse a ripartire in questi due generi le specie dell' oolite si fonda sulla diversa forma e disposizione dei nervi secondarj, secondo che all'uno o all'altro modo di distribuzione dei sori mi apparvero più favorevoli. IIo quindi posto nelle Taeniopteris tutte quelle specie che per la sottigliezza dei nervi e la loro frequente biforcazione più si approssimavano alla Taeniopteris Münsterii Gösp., considerando come la biforcazione dei nervi non porgesse ostacolo allo svolgimento dei sori, ove questi fossero collocati, come nelle Angiopteris, presso il margine della fronda lungo le ultime divisioni dei nervi che volgonsi parallele verso il margine stesso.

All'incontro, siccome nelle Danee la fruttificazione uccupa tutta la lunghezza dei nervetti secondarj, cosi riunii nel genere Danaeites tutte quelle forme in cui questi nervetti sono semplici, oppure una sol volta forcuti, ed alquanto fra di loro discosti in modo, che per Lutta la loro estensione, rimane libero tra l'uno e l'altro uno spazio, che sembra destinato ad accogliere la fruttificazione. Con tulto ciò, io non propongo questa ripartizione in due gruppi generici delle specie proprie dell'oolite, che in via affatto temporanea, allo scopo di richiamare su di essa l'attenzione degli studiosi, potendo venire in seguito confermata, modificata od anche contraddetla dal rinvenimento di esemplari forniti della frultificazione.

\section{Taeniopteris vittata Brong.}

T. fronde simplici, petiolata, lineari-lanceolata elongata, integerrima, ulrinque obtusa, $18-50$-millim. circiter lata, apicem versus altenuata; 


\section{$-202-$}

nervis secundariis tenuibus, e nervo medio subcanaliculato excurrenti, angulo recto egredientibus, horizontalibus, parallelis, 1-2-furcatis, interdum simplicibus.

Taeniopteris viltala. Brosg. Prodrom. pag. 62 ; Hist. Végét. foss. I. pag. 263 , tab. 82 , fig. 1. 2. 3. (excl. fig. 4.). - Lind. Hutr. Foss. Fl. Gr. Brit. I. pag. 175, tab. 62, fig. 1. 2.; et Vol. III. tab. 176 B. - Prese in Srfanis. Vers. Fl. Vorw. II. pag. 139. - Ung. Gen. et Spec. Pl. foss. pag. 213. - Mornis, Cal. Bril. foss. pag. 23. - Zigno, Enum. Filic. foss. form. Oolith. pag. 38.

Taeniopteris scitaminea. Presl in Sterma. Vers. Fl. Vorw. 1I. pag. 139. - Unger. Gen. el Spec. Plant. foss. pag. 212. - Morms, Cat. Bril. foss. pag. 23. - Zigno, Enum. Filic. foss. form. Oolith. pag. 39 .

Phyllites scitamineaeformis. Stensi. Fers. Fl. Vorw. I. pag. 39, lab. 37, fig. 2.

Aspidites Taeniopteris. Görr. Syst. Filic. foss. pag. 350.

Scolopendrium solitarium. Punc. Geol. Yorksh. I. pag. 125, tab. 8. fig. 5.

Scolopendrium. Yousg et Bird, Geol. Yorksh. pag. 182, lab. 2. fig. 7.

In stratis seriei oolithicae inferioris ad Cloughton, ad Gristhorpe et ad Stonesfield in Anglia.

Questa specie ha una fronda semplice, picciuolata, larga dai quindici millimetri ai tre centimetri e mezzo, e lunga dai quindici ai venti centimetri. La sua forma è lineare, lanceolata, allungata, ottusa tanto alla base, quanto all'apice, verso del quale leggermente si attenua. Il picciuolo ha un diametro di due a tre millimetri, e da esso si prolunga il nervo medio scanalato nel mezzo, che prosegue assottigliandosi fino all'estremità della fronda. I nervi secondarii assai soltili, escono ad angolo retto dal nervo primario e facendosi una o due volte forcuti, corrono orizzontali e paralleli fino al margine della fronda, ed a questi tratto tratto se ne osservano intercalati degli altri, affatto semplici.

Gli esemplari che servono di tipo per questa specie derivano dalle argille scistose della serie oolitica inferiore di Cloughton, di Gristhorpe e di Stonesfield in Inghilterra.

Le figure pubblicate da Phillips, Brongniart, Lindley ed Ifutton, e gli esemplari provenienti dall' Inghilterra che formano parte della mia collezione, chiariscono assai bene la forma generale ed i caratteri che distinguono questa specie. In quanto al saggio figurato nella Fossil Flora of Great Britain Vol. III. Tav. 176 B., la cui figura fu semplicemente ripetuta dallo Sternberg nella sua Flora der Vorwelt Vol. I. Tav. 37. Fig. 2., 


\section{- $203-$}

e a cui Presl diede il nome di Taeniopteris scitaminea, io lo considero un semplice frammento di questa medesima specie. La smarginatura che ne rende l'estremità obcordata, non può essere che accidentale, poichè ivi la rachide conserva una grossezza che certo non doveva avere nel punto estremo della fronda, per cui è a ritenersi che sia ivi rimasta troncata poco prima dell' apice. Anche i Signori Lindley e Hutton riferirono l'esemplare di Stonesfield alla Taeniopteris vittata del Brongniart, accennando però alla maggiore consistenza e larghezza della fronda ed alla piu fitta disposizione dei nervi nel saggio di Stonesfield. Ma fra gli esemplari della Taeniopteris vittata, ve ne sono alcuni che superano in larghezæa quello di Stonesfield e palesano una fronda consistente, pure munita di nervetti assai avvicinati.

Rimarrebbe solo la semplicità dei nervi, apparente dalla citata figura, clı potrebbe giustificare la separazione della Taeniopteris scitaminea dalla Taeniopteris viltala; ma di questo caraltere non fanno alcun cenno i Signori Lindley e Hutton, e d'altronde anche sugli esemplari della Taeniopteris vittata i nervi sembrano talvolta semplici, ed è mestieri esaminarli accuratamente colla lente per distinguerne le forcature. Che se questo carattere differenziale della semplicità dei nervi, realmente esistesse sull' esemplare di Stonesfield, gli autori della Flora fossile della Gran Rretagna non avrebbero certo ommesso di farne disegnare un ingrandimento che lo esprimesse.

Nelle opere in cui è trattato di questa specie se ne vede sovente citata la presenza non solo nella formazione oolitica d'Inghilterra, ma eziandio nei terreni liassici della Svezia, della Germania, della Baviera, dell'Austria e della Russia; ma è ancora assai dubbio se i saggi trovati nel Lias di quelle contrade, appartengano realmente alla specie stessa che si rinviene negli strati oolitici di Scarborough e di Stonesfield. E questo dubbio viene avvalorato dallo scorgere come il chiarissimo Professore Schenk nella sua bella Hora fossile degli strati interposti fra il Keuper ed il Lias abbia riunito alla Taeniopteris temuinervis Brauss, il Pterozamites scitamineus di Braun e quelle forme del Lias e dell'Infralias dell'Alemagna, che Andrae e Germar avevano riferito alla Taemiopteris viltata del Brongniart.

In quanto alla Taeniopteris trovata dall' Eichwald nei terreni liassici della Russia e del Caucaso, e che sotto il nome di Tacniopteris vittata egli descrisse e figurò nella sua Lethaea Rossica, Vol. II. pag. 24, Tav. 2. figg. 5., essa si mostra alquanto diversa dalla specie dell' oolite d'Inghilterra per avere la fronda che termina coll'estremita più acuta, e che inferiormente s'allenua, e decorre nel prolungamento della costa o nervo mediano che forma il picciuolo.

E pure da osservarsi, come l'esemplare trovato nell' arenaria liassica di llöer nella Scania, che fu riferito a questa specie e figurato nella Histoire des Végétaux fossiles 


\section{- $204-$}

alla tavola 82 , fig. 4., se ne palesi affatto diverso, avendo esso i nervi molto più grossi, più discosti fra di loro, e perfettamente semplici.

\section{Taeniopteris latifolia Brong.}

T. fronde 7-8-centim. circiter lata; nervo medio tereti crassiusculo; nervis secundariis, angulo subacuto egredientibus, subparallelis, horizontalibus, vel leviter arcuatis, fere omnibus furcalis.

Taeniopteris latifolia. Bnong. Prodrom. pag. 62; Hist. Végét. foss. I. pag. 266, tab. 82, fig. 6. - Sterna. Vers. Fl. Vorw. II. pag. 139. - Ung. Gen. et Spec. Plant. foss. pag. 213. - Monns, Cat. Bril. foss. pag. 23. - Zıgno, Enum. Filic. foss. form. Oolith. pag. 39.

Odontopteris latifolia. Stensi. Vers. Fl. Vorw. II. pag. 79

Zamites latifolius. Stenrib. Vers. Fl. Vorw. II. pag. 199.

In schisto argillaceo seriei oolithicae inferioris ad Stonesfield in Comitatu Oxford, Angliae.

La fronda è larga dai sette agli olto centimetri, ed è fornita di un nervo mediano, rotondo, sporgente, e segnato da qualche stria longitudinale. I nervi, al punto d'inserzione, sono un poco obbliqui, uscendo ad angolo quasi acuto dal nervo primario, e poscia facendosi orizzontali e leggermente arcuati procedono quasi paralleli fino al margine della fronda mostrandosi per lo più forcuti, ma talvolta intercalati da qualche nervo semplice.

Il saggio figurato dal Brongniart fu rinvenuto nello scisto argilloso, spettante alla serie oolitica inferiore, di Stonesfield nella Contea d' Oxford nell'Inghilterra.

Il frammento di cui l'illustre Brongniart diede la descrizione e la figura, ha la fronda trasversalmente fessa in guisa da farla sembrare pinnatifida, ed è perciò che nell'opera dello Sternberg la veggiamo collocata anche nel genere Odontopteris e persino fra le Cicadeacee nel genere Zamites. Ma solo che si ponga mente alla direzione dei nervi, sarà agevole convincersi che un tale aspetto è dovuto ad accidentale lacerazione del lembo della fronda.

Se esemplari più completi giungeranno a meglio stabilire i caralteri distintivi di questa specie, sarà allora necessario di cangiarne il nome. Imperciocchè dopo l'epoca 
in cui fu pubblicata l'Opera del Brongniart si sono scoperte delle Taeniopteris che hanno una fronda di gran lunga più larga della presente.

\section{Taeniopteris Villiamsonis Göpp.}

T. fronde simplici, lanceolato-oblonga, utrinque obtusa, margine subrepando, $\mathbf{b}$-centim. circiter lata; nervo medio crassiusculo, striato-sulcato, attenuato, sub apice frondis evanescente; nervis secundariis tenuibus, remoliusculis, angulo subrecto egredientibus, parallelis, subhorizontalibus 2 $\mathbf{3}$-furcatis.

Taeniopteris Williamsonis. Görp. Gen. Plant. foss. Liv. 3. 4. pag. 51. - Zıgro, Enı . Filic. foss, form. Oolith. pag. 39.

Taeniopteris major. Jinds. Hutr. Foss. Fl. Gr. Brit. II. pag. 31, tab. 92. - Sternв. Vers. Fl. Vorw. II. pag. 140. - Ung. Gen. et Spec. Plant. foss. pag. 212. - Monms, Cat. Brit. foss. pag. 23.

Aspidites Williamsonis. Görr. Syst. Filic. foss. pag. 353.

In schisto argilloso formationis oolithicae ad Gristhorpe et ad Cloughton, prope Scarborough, Angliae.

Il saggio figurato nell'Opera dei Signori Lindley e Hutton presenta un buon tratto della fronda, mancante però di tutta la parte inferiore. All'incontro, in un esemplare della mia collezione manca invèce l'estremità superiore. Questi due saggi porgono elementi bastevoli per riconoscere la forma e larghezza della fronda e per calcolarne approssimativamente la lunghezza. La fronda si palesa quindi lanceolata, bislunga, ottusa tanto all'apice cle alla base, col margine lievemente ondeggiato, larga cinque centimetri e mezzo, e probabilmente non più lunga dei dieciotto centimetri. Essa è fornita di un nervo primario, che al basso ha un diametro di due millimetri e poscia procede attenuandosi fino a che svanisce poco sotto l'apice della fronda. Esso è finamente striato e percorso da un solco longitudinale. I nervi secondarj sono sottili, alquanto fra loro discosti, e partendo ad angolo retto dal nervo mediano corrono paralleli ed orizzontali, biforcandosi due o tre volte, fino al margine della fronda. Però nel terzo superiore di questa e fino alla sua estremità prendono una direzione un po'obbliqua, uscendo ad angolo men retto dal nervo primario.

Questa specie è annoverata dal Sig. Leckenby fra quelle che sono comuni tanto ai depositi di Gristhorpe che a quelli di Cloughton che giaciono nella formazione oolitica della Contea di York in Inghilterra. 
Il nome di Taeniopteris major, accennando ad un carattere che non può più ammettersi in questa specie, dopo che altre ne furono scoperte colla fronda assai piu grande, cosi ho creduto di ritenere quello datole dal Göppert, quando la annoverò fra le sue Aspidites, e che poscia le conservò ritornandola al genere Taeniopteris, come si legge nelle generalità premesse alla sua descrizione della Taeniopteris Mïnsterii, nell'opera intitolata, Les Genres des Végétaux fossiles. Liv. 3. 4. pag. 51.

\section{Taeniopteris ovalis Presl.}

T. fronde simplici? 5-centim. lata, $\mathbf{3}$-centim. longa, breviter petiolata, ovato-subelliptica, integerrima, utrinque acutiuscula; nervo medio filiformi, usque ad apicem excurrente; nervis secundariis tenuibus, angulo subrecto egredientibus, parallelis, subhorizontalibus, furcatis simplicibusque alternantibus.

Taeniopteris ovalis. Prest. in Sterre. Vers. Fl. Voru. II. pag. 141. - Ungre, Gen. el Spec. Plant. foss. pag. 213. - Monns, Cal. Bril. foss. pag. 23. - Zıgno, Enum. Filic. foss. form. Oolith. pag. 39.

Otopteris ovalis. Lisd. Ilutr. Foss. Fl. Gr. Brit. IlI. tab. $210 \mathrm{~A}$.

In formatione oolithica ad Gristhorpe Bay prope Scarborough in Anglia.

Non ho potuto avere saggi di questa specie, e quindi la descrivo attenendomi alla figura che ne diedero i Signori Lindley e Hutton solto il nome di Otopteris ovalis. Essa vi apparisce brevemente picciuolata, di forma ovato-ellittica col margine intero e con ambe le estremita alquanto acute, e misura cinque centimetri in lunghezza sopra tre di larghezza. Il nervo mediano è filiforme, e si protende fino all'apice, ed i nervetti secondarj, assai tenui, escono da quello ad angolo quasi retto e proseguono paralleli e quasi orizzontali, facendosi una sol volta forculi ed alternando con dei nervetti semplici.

Questa specie fu trovata nella formazione oolitica a Gristhorpe Bay presso Scarborough nell' Inghilterra.

I Signori Lindley e IIutlon avvertono che un ailtro esemplare di assai minore dimensione, fu rinvenuto nello stesso luogo, ed esternano il sospetto, che questi due saggi possano avere appartenuto ad una fronda pinnata; e su questo semplice cenno, Presl e 


\section{- 207 -}

Unger nelle loro descrizioni attribuiscono a questa specie una tal forma. Io invece propenderei a credere che fossero frondi giovani di alcuna delle precedenti specie, ma non avendo dati sufficienti per sostenere un tal parere, mi limitai a ritenere la fronda senplice, apponendovi però un punto interrogativo in segno di dubbio.

Nei terreni mesozoici delle Indie si rinvennero alcune forme che furono riferite a questa specie, e che, a dir vero, nell'aspetto generale presentano con essa molta affinità. Però, esaminando scrupolosamente le figure da cui è accompagnata la bellissima 0pera del chiarissimo Prof. Oldham di Calcutta (Palaeontologia Indica, tab. III. fig. 3. 4. 5. 6.), vi si rimarcano alcune differenze che farebbero sospettare, essere la specie Indiana diversa da quella d'Inghilterra. La fronda, negli esemplari delle Indie, ha una forma piì ottusa. Il nervo mediano, anzichè essere filiforme, giunge nella parte inferiore ad un diametro di due millimetri, si attenua assai lentamente, ed è segnato nel mezzo da una stria longitudinale. I nervetli secondarj escono da quello ad angolo acuto, e sono quasi tutti forcuti e ciò fin dalla base o poco sopra di essa, mentre nella specie di Gristhorpe Bay, i nervetti forcuti alternano con dei nervicciuoli semplici e le forcature cominciano per lo più alla metà della loro lunghezza.

\section{Ordo XVI. DaNAEACEAE.}

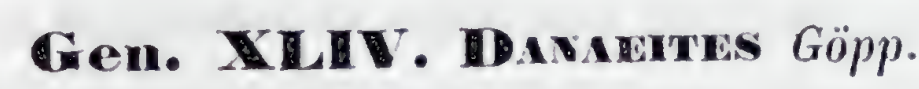

Frons petiolata, coriacea, simplex vel pinnata. Nervus primarius (Costa), crassus, excurrens. Nervi sccundarii, angulo recto vel aculo egredientes, paralleli, remoliusculi, simplices vel basi furcati, apice liberi vel arcuatim confluentes. Sporangia juxta nervulos biscriala, per totam nervulorum longitudinem, in soros lineares connata, apice poro orbiculari instructa.

Görr. Syst. Filic. foss. pag. 380 (emend.). - Uvg. Gen. el Spec. Plant. foss. pag. 211 (emend.). - Etringsn. et Debex, Urw. Acrobr. Kreidegeb. Aach. in Denksch. K. Acal. Wien, Vol. XVI. pag. 202 (emend.).

Taeniopteris. Brosg. Prodrom. pag. 61. Hisl. Végél. foss. I. pag. 262 (ex parte). - Prese in Strang. Vers. Fl. Vorw. II. pag. 138 (ex parte). - Uxa. Gen. el Spec. Plant. foss. pag. 211 (ex parte).

Aspidites. Göpr. Syst. Filic. foss. pag. 348 (ex parte).

Danacopsis. IIter, Urw. d. Scheweiz. pag. 54. 


\section{Danacites Mecrii Zigno.}

Tav. XXV. fig. 1.2. 3. 4.

D. fronde coriacea petiolata, simplici, integerrima, late lineari-lanceolata, obtusa, 70-centim. circiter longa, 7-centim. lata, apicem versus sensim angustata, basi in petiolum altenuata; costa crassissima obtuse striato-costata, 10-milim. lata, dein gradatim attenuata et usque ad apicem frondis excurrente; nervis crassis, simplicibus sub angulo recto egredientibus, parallelis, horizontalibus, remotiusculis, basi sinu semirotundo discretis, apicibus, ad marginem frondis, arcuatim confluentibus.

Taeniopteris Heerensis. Zigno, Enum. Filic. foss. form. Oolith. pag. 39 (Err. Iyp.).

Taeniopteris Beggialiana. Zigno, loc. cit. pag. 39.

In saxo calcareo cinereo, seriei oolithicae inferioris ad Val d'Assa prope Rotzo in Provincia Vicentina, nec non ad Bienterle prope Selva di Progno in Provincia Veronensi.

La fronda è picciuolata, semplice, interissima, di consistenza coriacea e di forma largamente lineare lanceolata, che lentamente si ristringe verso l'apice che è ottuso, attenuata verso la base e decorrente nel picciuolo. La sua maggiore larghezza arriva ai sette centimetri, ed in quanto alla sua lunghezza l'esame di varii esemplari mi fa arguire che giungesse all'incirca ai settanta centimetri. It picciuolo si prolunga superiormente nella costa mediana assai grossa ed ottusamente strialo-costata, il cui diametro, al basso, misura 10 millimetri, la quale poscia assai lentamente assottigliandosi scorre fino all' apice della fronda ove palesa ancora, nel punto estremo, una grossezza di circa due millimetri. Dai lati di essa escono ad angolo retto i nervi secondarj, semplici e piuttosto robusti, separati alla base da un seno semicircolare e discosti tra di loro circa un millimetro, che paralleli ed orizzontali si protendono ritti fino al margine della fronda, ove le loro estremità confluiscono formando una breve curva omologa al seno che dal lato opposto li disgiunge nel punto d'inserzione sulla costa.

Gli esemplari di questa specie provengono dagli strati della calcarea grigia spettante alla serie oolitica della Val d'Assa presso Rotzo nei Sette Comuni del Vicentino e da quelli di Bienterle in comune di Selva di Progno nella Provincia di Verona. 
L'accurato esame di varj saggi mi convinse che la specie da me descritta sotto il nome di Taeniopteris Beggiatiana, nella Enum. Filic. foss. form. Ootith. pag. 39 non è che la porzione inferiore della fronda della Taeniopteris Heerii. Devo pure avvertire che nel detto Elenco fu per errore stampato, T. Heerensis Zig., in luogo di T. Heerii Zra., e lo stesso avvenne rispetto alla Gycadopteris Heerii Zig. Vedi Enum. Filic. etc. pag. 31.

\section{Danacites Brongniartiana Zigno.}

Tav. XXV. fig. 5. 6 .

D. fronde ampla, simplici, integerrima, convexiuscula, ovato-oblonga, apice rotundata, 9-centim. lata; costa crassa prominente irregulariter striatosulcata, 6-millim. circiter lata, sensim altenuata et usque ad apicem frondis excurrente; nervis crebris, simplicibus, rigidiusculis, angulo recto vel subrecto e costa egredientibus, horizontalibus, parallelis, approximatis, sinu angusto obtuso basi interstinctis, apicibus ad marginem frondis arcuatim confluentibus.

Taeniopteris Brongniarliana. Zıano, Enum. Filic. foss. form. Oolith. pag. 40.

In saxo calcareo lutescente, seriei oolithicae inferioris ad Montem Raut prope Selva di Progno in Provincia Veronensi.

Questa forma ha pure una fronda semplice col margine interissimo, ma dall'impronta apparisce fosse alquanto convessa. Essa è ampiamente ovata, bislunga, nè gran fatto si attenua verso l'apice, ma termina ivi coll'estremità assai rotondata. La sua larghezza giunge ai nove centimetri. In quanto alla lunghezza essa non può calcolarsi sull' unico esemplare fin qui trovato, poichè sebbene sia lungo ventitre centimetri manca però inferiormente di gran parte della fronda. La costa che la percorre è robusta e prominente, irregolarmente striato-solcata ed ha un diametro di circa sei millimetri che minuisce gradatamente proseguendo fino all'apice della fronda ove termina conservando ancora la grossezza di un millimetro. I nervetti escono dalla costa mediana ad angolo retto o quasi retto, e sono pure paralleli ed orizzontali ma più numerosi, più avvicinati tra loro, e più sottili di quelli della specie precedente. Alla base sono separati da un seno ottuso molto più angusto, ma colle loro estremità confluiscono egualmente fra di loro giunti al margine della fronda. 
Questa bella specie fu trovata una sol volta nella calcarea giallognola del Monte Raut in Comune di Selva di Progno nel Veronese.

Al genere Danaeites sembra pure che possano essere riferite le foglie scoperte dal chiarissimo Ugo Miller, nei terreni oolitici di Helmsdale, nella Contea di Sutherland in Iscozia, e che dai frammenti raccolti, egli calcolò potessero aver raggiunto una lunghezza di ben trenta pollici sopra cinque di larghezza. Nella sua Opera intitolata: The testimony of the Rocks pag. 438, egli si linita ad accennare alle loro analogie colle Taeniotteridi, rimarcando come siano semplici, coriacee, munite di una grossa costa longitudinale nel mezzo, da cui escono ad angolo retto, dei nervi semplici che corrono orizzontali fino al margine della fronda.

\section{Ordo XVII. Schizencene.}

\section{Gen. Xla. SThonvetenis Pomel.}

Frons pluries pinnata; pinnulis ovatis, integris, basi angustis; nervis obsoletis. Fructificatio spicaeformis, summis rachibus inserta, squamis distichis, alternis, imbricatis, constans.

Ponel, Maler. p. sero. a la Fl. foss. ter. jurass. de la France; in Amll. Ber. Versamml. Deutsch. Natur. u. Aerz. Aachen. 1847, pag. 336.

Il genere Stachypteris di Pomel, che nell'Enum. Filicum foss. form. Oolithicae io aveva posto temporaneamente in un gruppo separato, che chiamai delle Stachitteridee, presenta a dir vero nella fruttificazione caratteri sufficienti per potere ammettere la sua affinità con taluna delle forme che sono comprese nella famiglia delle Schizeacee.

E bensì vero che anche nelle Osmundacee la fruttificazione è posta egualmente sulle rachidi estreme della fronda, ma terminale è pure quella delle Schizee, e d'altronde, come avvertiva il Pomel, la fruttificazione delle Stachypteris si palesa molto analoga a quella dei Lygodium, per la identica forma e disposizione delle squamme che distiche ed embricate compongono la spica. Per queste osservazioni credo più opportuno il collocare per ora le Stachypteris fra le Schizeacee, anzichè farle servire di tipo per una nuova famiglia. 


\section{Stachypteris spicans Pomel.}

S. fronde 2-5-pinnata, rachide stricta, gracili, subflexuosa; pinnis patentibus elongatis; pinnulis discretis, frondis basilari parte pinnatim multilobatis, media parte tantum quinque-trilobatis, superioribus simplicibus, pinnulam majoris ordinis, praecedentibus similem, efficientibus; lobis basi plus minusve discretis, obovatis, basi angusta subdecurrentibus, minutis, terminali aliis conformi; nervulis obsoletis; spicis fructiferis lineari-oblongis, obtusis, frondis summae pinnarum pinnularumque superiorum, rachim terminantibus.

Stachypteris suicans. Pomer, Mat. p. serv. a la Fl. foss. ter. jurass. de la France, in opere cit. pag. 336, n. 4. - Zıgno, Enum. Filic. foss. form. Oolith. pag. 40.

In stratis seriei oolithicae mediae ad S.' Michel in Gallia.

La fronda è due o tre volte pinnata, colle pinne patenti ed allungate, munita di pinnule disgiunte fra loro, e di una rachide gracile ed alquanto flessuosa. Le pinnule della parte inferiore della fronda sono pinnato-multilobate, quelle della parte media, solo divise in cinque lobi, semplici quelle della parte superiore. I lobi delle pinnule sono più o meno separati fra di loro, di forma obovata un po' decorrenti nella base, che è angusta, e col lobo terminale eguale agli altri. La fruttificazione è disposta in ispiche bislunghe, oltuse, collocate sulla parte estrema delle rachidi, che sorreggono le pinne e le pinnule superiori della fronda.

Questa curiosa Felce fu trovata nel terreno coralliano di S.' Michel in Francia.

\section{Stachypteris litophylla Pomel.}

S. fronde, 5-(4?) pinnata, rachide angusta, stricta; pinnis angulo acuto divergentibus, remotis; majoribus, pinnulis lineari-oblongis, distantibus, lobis ovatis minulis.

Stachypteris litophylla. Porex. Loc. cit. pag. 337, n. 2. - Zigro. Enum. Filic. foss. form. Oolith. pag. 40.

Eodem in loco cum praecedenti. 


\section{$-212-$}

Si distingue dalla Stachypteris spicans per una fronda quasi tripinnata, e per le pinne remote e divergenti ad angolo acuto, di cui le maggiori son fornite di pinnule distanti, lineari-oblunghe, coi lobi minuti e di forma ovata.

Iu trovata nel medesimo luogo colla antecedente, e quantunque l'esemplare manchi degli organi della fruttificazione, tuttavia l'analogia presentata dalla forma generale della fronda, indusse il Pomel a comprenderla in questo genere.

\section{Stachypteris pulchrar Pomel.}

S. fronde..., lobis ovato-lanceolatis; spicis minoribus et angustioribus, apice subattenuatis obtusis.

Stachypteris puIchra. Ponee, Loc. cil. pag. 337, n. 3. - Zigno, Enum. Filic. foss. form. Oolith. pag. 40.

In stratis corallianis formationis oolithicae mediae apud Chateauroux in Gallia.

Di questa specie il Pomel rinvenne un solo frammento, però munito della fruttificazione. Esso consta di una sola pinnula, o piuttosto di un lobo ovale un poco lanceolato, e più grande di quelli della Stachypteris spicans. Le spiche poi si manifestano, nella presente specie, più strette, più lineari, e sensibilmente attenuate all'estremità.

Questo saggio fu rinvenuto nel terreno coralliano presso Chateauroux in Francia.

\section{Classis V. LYCOPODIACEAE.}

Le Licopodiacee, che si svolsero con si rigogliosa vegetazione durante l'epoca Paleozoica, e popolarono degli svariati e numerosi loro resti le stratificazioni dei terreni Siluriano, Devoniano e Carbonifero, scemarono grandemente nell' epoca Mesozoica, mancando ogni vestigio di loro nella formazione Triasica, e ricomparendo poscia con poche specie nel Lias, e con quattro sole nell'Oolite. Imperciocchè dobbiamo escludere da questa Classe il Lycopodites uncifolius PhIL., o Lycopodites Williamsonis Brong., cosi frequente nei terreni oolitici dell'Inghilterra, che l'illustre Brongniart fino dal 1849 
sospettava appartenesse alle Conifere e proponeva di collocare nel genere Palissya, e che realmente devesi considerare una Conifera, la quale, per le recenti osservazioni del Prof. Schenk, sarebbe molto affine alle Arancarie.

Le quattro forme dei terreni oolitici, che si troveranno qui descritte, sono ripartite nei generi Lycopodites, Psilotites, Isoetites; nel che ho seguito il parere de' più riputati autori, quantunque, toltone il Lycopodites falcatus Lindu. Hurt., le altre, a dir vero, non ci porgano tali analogie coi generi cui furono riferite, da poterle risguardare in via assoluta, quali rappresentanti fossili dei generi Psilotum ed Isoetes.

\section{Ordo XVIII. Licopodiene.}

\section{Gen. XU. Lucopontes Brong.}

Caulis dichotome ramosus. Rami alterni dichotomi; foliis polystichis vel distichis seriebus oppositis, cicatrices vix distinctas relinquentibus.

Brong. Prodrom. pag. 83 (ex parte); Tabl. Gen. Végél. foss. pag. 40. - Ung. Gen. el Spec. Plant. foss. pag. 273 (ex parte).

\section{Lycopodites falcatus Lindl. Hutt.}

L. caule gracili, filiformi, dichotome ramoso, ramis alternis dicholomis; foliis inacqualibus, distichis, sessilibus, integerrimis, ovato-lanceolatis, falcatis, apice attenuatis obtusis.

Lycopodites falcalus. Lindu. Ilutr. Foss. Fl. Gr. Brit. I. pag. 172, tab. 61, fig. 1. 2. - Uxa. Gen. et Spec. Plant. foss. pag. 274. - Monn1s, Cat. Brit. foss. pag. 12.

Muscites falcatus. Sтевкr. Vers. Fl. Vorw. II. pag. 38.

In formatione oolithica ad Cloughton prope Scarborough, Angliae.

Un caule gracile, filiforme, fornito di foglie e che si ramifica in forma dicotoma distingue questa pianticella. I rami sono alterni e si dicotomizzano più d'una volta. Le foglie non sono tutte di eguale dimensione; sono sessili, distiche, o quasi opposte, col margine interissimo, e prive di alcuna traccia di nervi, di forma ovato-lanceolata, falcala, inferiormente larghe, superiormente attenuate, incurve ed ottuse all'apice. 


\section{- $214-$}

Questa specie proviene dai terreni oolitici di Cloughton presso Scarborough nell' Inghilterra. I Signori Lindley e IIutton nella Fossil Flora of Great Britain, riferirono alla presente specie una informe figura della Tav. II. della Geological Survey of the Yorlishire Goast dei Signori Young e Bird, in cui la ramificazione è invece pinnata, i rami semplici, le foglie anguste e senza alcuna curva verso l'estremità. Per quanto possa riternersi rozza la figura, data dai Signori Young e Bird, è però sufficiente a farci conoscere, ch'essa non può rappresentare la forma, cui i Signori Lindley e Itutton, dicdero poscia il nome di Lycopodites falcatus.

\section{Ordo XiX. Psiloteae.}

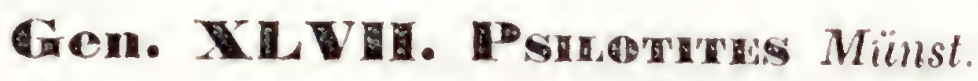

Cormus (Rhizoma) cylindricus oblongus. Caulis longitudinaliter striatus, squamis alternis instructus. Folia dichotoma.

Müsst. Beilr. V. pag. 108. - Brosa. Tabl. Gen. Végét. fass. pag. 41. - Broxx, Lethaca Geogn. II. Pars IV. pag. 58.

\section{Pilotites filiformis Münst.}

P. rhizomate cylindrico, obovato rotundato, basi truncato; caule squamoso simplici, erectiusculo, striato-sulcato, squamis alternis, superne altenuato filiformi, apice foliis capillaribus, dichotomis, brevissimis instructo.

Psilotites filiformis. Mürst. Beitr. V. pag. 108, tav. 13, fig. 11; tav. 15, fig. 20. - Ung. Gen. et Spec. Plant. foss. pag. 279 (inter Lycopod. dubias). - Brosw, Lethaea Geogn. Vol. II. Pars IV. pag. 59, tav. 142, fig. 7. a, 6. - Fruscnur. Vers. Zusamm. foss. Th. u. PRanz. d. Lithogr. Kalk. in Bayern. pag. 44.

In calcareo lithographico seriei oolithicae superioris ad Daiting prope Monheim in Bavaria.

Il rizoma è cilindrico, ha un diametro di quattro millimetri, ed è lungo dodici. Esso è di forma obovata, tronco nell' estremità inferiore e rotondato all'apice, ove sorge il caule alquanto eretto, semplice, lungo ventidue millimetri, striato-solcato, che osservato colla lente si palesa fornito di piccole squamme alterne e brevissime, simili a 
quelle dei Psilotum della Flora attuale. Il caule si attenua e diventa filiforme progredendo verso l'estremità ove si risolve in foglioline capillari e dicotome, assai brevi.

Questa forma descritta e figurata dal Münster e dal Bronn si rinvenne nel calcare litografico di Daiting presso Monheim nella Baviera.

Le affinità di questa pianticella coi Psilotum si limitano alle apparenze presentate dal caule e sopratutto alle piccolissime squamme che vi si riscontrano; poichè del resto le sue dimensioni quasi microscopiche, come giustamente osserva il Brongniart, impediscono di accogliere in modo positivo la sua appartenenza a quel genere.

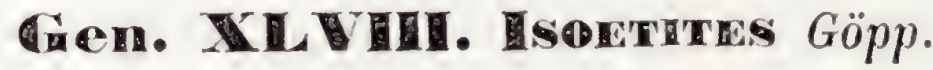

Cormus simplex depressus, foliis simplicibus, linearibus subulatis instructus.

Görp. in Gens. Mineral. 438. - Bnong. Tabl. Gen. Végét. foss. pag. 40. - Ux́, Gen. et Spec. Plant. foss. pag. 225. - Bnows, Lelhaea Geogn. Vol. II. Pars IV. pag. 58.

\section{Isoctites crociformis Münst.}

I. cormo simplici depresso bulbiformi, infra truncato, supra foliis linearibus erectis, basi unilis, apice subulatis, obsesso.

Isoctites crociformis. Müsst, Beilr. V. pag. 107, tab. 4, fig. 4. - Brong. Tabl. Gen. Végét. foss. pag. 40. - Ungen, Gen. el Spec. Plant. foss. pag. 40. - Bross, Lethaca Geogn. II. Pars IV. pag. 58, lab. 14.", fig. 6. - Friscus. Vers. Zusamm. foss. Th. u. Pfanz. d. Lithogr. Kalk. in Bayern. pag. 44 .

Cum praecedenti in calcareo lithographico seriei oolithicae superioris ad Daiting prope Monheim in Bavaria.

Da un fusto semplice, bulbiforme, dilatato e tronco alla base, ove ha un diametro di quattordici millimetri, si svolge superiormente un fascio di foglie, unite alla base, di forma lineare e subulate all'apice, di cui le maggiori hanno un millimetro e mezzo di diametro sopra una lunghezza di tre centimetri. 
Come vedemmo questa pianticella, trovata in Baviera a Daiting presso Monheim in un terreno ora riferito alla serie oolitica superiore, è ritenuta dagli autori quale una forma analoga alle Isoetes della Flora attuale; e sebbene il suo aspetto risvegliar possa anche l'idea che essa rappresenti soltanto lo stato giovane di qualche altra forma vegetale, tultavia non si saprebbe per ora riscontrarvi affinità maggiori con altri generi.

\section{Isoetites Murrayana Ung.}

I. cormo depresso, globuloso, foliis basi confertis, linearibus, subulatis, fistulosis, ancipitibus, striatis, enerviis.

Isoetiles Murrayana. Unger, Gen. et Spec. Plant. foss. pag. 226. - Bnons, Lethaca Geogn. II. Pars IV. pag. 58.

Solenites Murrayana. Lixdu. Hutr, Foss. Fl. Gr. Brit. II. pag. 105-109, tab. 121. - Monnıs, Cat. Brit. foss, pag. 20.

Flabellaria viminea. Primliss, Geol. Yorksh. I. Lab. 10, fig. 12.

In formatione oolithica ad Redcliffe et ad Gristhorpe prope Scarborough, Angliae.

Gli esemplari di questa forma, comune nell'argilla scissile della serie oolitica inferiore a Gristhorpe nell'Inghilterra, ci presentano un ammasso di foglie aggruppate alla base sopra una specie di fusto, le quali poscia s'allungano in forma lineare coll'apice subulato, larghe appena un millimetro, fistolose ed ancipiti nella loro sezione trasversale, prive di nervi, ma striate per lo lungo e talvolta leggermente curvate.

Sebbene questa pianticella lasci ancor molto da desiderare per ben conoscere le sue reali aftinità, pure non v'ha dubbio che essa molto si avvicini alle Isoetes dell'epoca attuale. 


\section{SPIEGAZIONE DELLE TAVOLE}

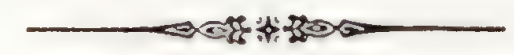

TAv. I.

Fig. 1-2. Confervites Veronensis Zigno, pag. 6. - Fig. 3. Codites Krantzianus Zigno, pag. 10. Fig. 4. a, b. Trevisania furcellata ZIGNo, pag. 23. - Fig. 4.* Chondrites Targionii STERnв. pag. 28.

TAv. II.

Fig. 1. Chondrites furcatus STERno. pag. 30. Fig. 2. Chondrites intricatus STERnв. pag. 31. - Fig. 3. Sphaerococcites Meneghinii Zigno, pag. 33. Fig. 4. Sphaerococcites Savii Zigso, pag. 34. Fig. 5-5, a. Granularia linearis ZigNo, pag. 37.

TAV. III.

Fig. 1. Calamites Beanii Buns. pag. 45. - Fig. 2. Equisetites Bunburyanus ZigNo, pag. 62. - Fig. 3. Calamites lateralis Zigno, pag. 46. - Fig.4-5. Equisetites Bunburyanus Zigno, pag. 62.

\section{TAv. IV.}

Fig. 1-6. Equisetites Bunburyanus ZigNo, pag. 62. Esemplari provenienti dal Monte Pernigotti nel Veronese.

\section{TAY. V.}

Fig. 1-4. Equisetites Bunburyanus ZigNo, pag. 62. Esemplari della maggiore grandezza trovati sul Monte Raut nel Veronese.

\section{TAv. VI.}

Fig. 1-6. Equisetites Veronensis Zigno, pag. 64 .

$$
\text { TAv. VII. }
$$

Fig. 1-6. Phyllotheca Brongniartiana Zigno, pag. 59. - Fig. 7. Ingrandimento che fa vedere come l'inserzione dei rami sia superiore alla guaina. -
Fig. 7. $a, b, c$. Ingrandimenti delle guaine $e$ delle lacinie.

$$
\text { TAv. VIII. }
$$

Fig. 1-5. Phyllotheca equisetiformis Zigno, pag. 60. - Fig. 5., a. Ingrandimento di una guaina. - Fig. 6-7. Ricostruzione a semplice contorno della stessa specie.

\section{TAV, IX.}

Fig. 1.-1, a. Cyclopteris minor Zigno, pag. 106. - Fig. 2.-2, a. Protortipis Asarifolia Zigno, pag. 180. - Fig. 3.-3, a. 4.-5.-5, a. Hymenophyllites Leckenbyi ZiGNo, pag. 95.

$$
\text { Tav. X. }
$$

Fig. I. Gleichenites elegans ZiGNo, pag. 193. Fig. 1., a. Ingrandimento di porzione di una pinna, che fa vedere il modo con cui si dicotomizza e la forma e disposizione alterna dei segmenti. - Fig. 2. Frammento della stessa specie.

$$
\text { TAV. XI. }
$$

Fig. 1-2. Odontopteris Ungeri ZIGNo, pag. 110. Esemplari del Monte Pernigotti. - Fig. 3. Esemplare della stessa specie proveniente dalla calcarea grigia di Rotzo. - Fig. 3., a. Ingrandimento che fa conoscere la distribuzione dei nervi.

$$
\text { TAv. XII. }
$$

Fig. 1. Uno dei due rami in cui è partita la fronda della Dichopteris Visianica Zigno, pag. 116, rappresentato nella sua naturale grandezza. - Fig. 1., a. Ingrandimento che fa vedere la forma delle pinnule o segmenti, ma in cui per errore $i$ nervetti si segnarono più numerosi del vero. 


\section{TAv. XIII.}

Fig. 1. Dichopteris Visianica Zigno, pag. 116. Ridotta alla metà della grandezza dell' esemplare. - Fig. a. Forma e grandezza delle pinnule. - Fig. b. Vera disposizione dei nervetti.

\section{TAv. XIV.}

Fig. 1. Hymenophyllites Leckenbyi ZiGNo, pag. 95. - Fig. 1., b. Ingrandimento di una pinna. Fig. 2. Dichopteris lanceolata Zigno, pag. 118. Fig. 3. Dichopteris laevigata ZiGno, pag. 118. - Fig. 4. Dichopteris Paroliniana Zigno, pag. 120.

\section{TAV. XV.}

Fig. 1-3. Dichopteris angustifolia ZIGNo, pag. 121. - Fig. 4. Dichopteris rhomboidalis $Z_{\mathrm{IGNO}}$ pag. 122. - Fig. 5., a. Dichopteris microphylla Zigno, pag. 122. - Fig. 5., 6 . Una pinnula ingrandita per far vedere i sori, la cui grandezza fu però esagerata dal disegnatore. - Fig. 6. Pinnula ingrandita della Dichopteris Paroliniana ZIGNo, pag. 120.

\section{TAV. XVI.}

Fig. 1-2. Cycadopteris Brauniana ZIGNo, pag. 155. - Fig. 3-4. Ingrandimenti che fanno vedere la disposizione e forma dei sori. - Fig. 5. Distribuzione esatta dei nervi. - Fig. 6. Fronda giovane della stessa specie.

\section{TAv. XVII.}

Fig. 1. Fronda della Cycadopteris Brauniana $\mathrm{ZI}_{\mathrm{I}}$ GNo, giunta al suo pieno accrescimento. - Fig. 2. Ingrandimento in cui si scorgono i sori e la disposizione dei nervi. - Fig. 3. Cycadopteris undulata Zigivo, pag. 157. - Fig. 4. Rovescio di una porzione di pinna da cui si rileva la grossezza del nerro mediano. - Fig. 5. Ingrandimento di una pinna che fa vedere l'ondulazione del margine ed il modo d'inserzione sulla rachide.

\section{TAv. XVIII.}

Fig. 1. Cycadopteris heterophylla ZiGNo, pag. 158. - Fig. 2. Pinna ingrandita che palesa la distribu- zione dei nervi. - Fig. 4. Esemplare del calcare litografico di Nussplingen. - Fig. 5. Individuo giovane della stessa specie.

\section{TAv, XIX.}

Fig. 1.-1, a.-2. Cycadopteris Heerii ZiGNo, pag. 160. - Fig. 1. b. Pinna ingrandita. - Fig. 3.-5. Frondi fertili della Marzaria Paroliniana Zigno, pag. 170. - Fig. 3., a.-3, b. Ingrandimenti che fanno vedere la distribuzione dei nervi e dei sori. - Fig. 6.-7. Frondi sterili della stessa specie.

\section{TAv. XX.}

Fig. 1. Sagenopteris angustifolia $\mathrm{Z}_{\mathrm{IGNO}}$ pag. 186. - Fig. 2-3. Esemplari nella cui vicinanza si osservano dei corpicciuoli rotondi colla superficie munita di piccole asperitì, che potrebbero forse rappresentare la fruttificazione di queste pianticelle. Fig. 4-5-6-7. Saggi diversi della stessa specie, fra i quali deve rimarcarsi che il $n 0^{\circ} 5$. ha cinque pinne invece di quattro. - Fig. 8-9. Frondi giovani della stessa specie. - Fig. 10. Ingrandimento in cui fu troppo esagerata la regolarità delle areole, ed in cui i nervi sono segnati assai più sottili del vero. - Fig. 11. Sagenopteris cuneata Morris, pag. 183. Esemplare della calcarea giallognola del Monte Pernigotti nel Veronese.

\section{TAv. XXI.}

Fig. 1. Pinna laterale della Sagenopteris Goeppertiana ZiGNo, pag. 188. - Fig. 1., a. Ingrandimento che spiega la esatta distribuzione dei nervi e la brevità del nervo mediano. Fig. 2-3. Frondi giovani della medesima specie. - Fig. 4-5. Pinne mediane della Sagenopteris Goeppertiana ZIGNo, giunta al suo pieno accrescimento. - Fig. 6., a. Sagenopteris reniformis ZIGNo, pag. 184. - Fig. 6., b. Distribuzione dei nervi della stessa specie.

$$
\text { TAV. XXII. }
$$

Fig. 1-2. Sagenopteris Goeppertiana Zigno, pag. 188. - Fig. 3., a. Laccopteris Rotzana ZigNo, pag. 197. - Fig. 3., b. Ingrandimento di una pinna. Fig. 3., c. Ingrandimento di una pinnula che palesa la disposizione dei nervi e dei sori. 


\section{$-219$}

TAv. XXIII.

Fig. 1. Dictyophyllum Leckenbyi Zigno, pag. 178. - Fig. 1., a. Ingrandimento. - Fig. 2. Dictyophyllum rugosum Linde. Hutr. pag. 176. - Fig. 2,a a. Ingrandimento. Ho creduto opportuno di dare una esatta figura di questa specie, perchè non ne ho trovata alcuna fra le pubblicate, in cui fosse esattamente espressa la vera distribuzione dei nervi.

TAv. XXIV.

Fig. 1.-2.-3. Ctenis falcata Lindu. Hutr. pag. 190. - Fig. 1., a. Ingrandimento. Anche di questa specie ho voluto figurare alcuni esemplari da me posseduti, allo scopo di far meglio conoscere la disposizione dei nervi.

\section{TAv. XXV.}

Fig. 1. Danaeites Heerii Zigno, pag. 208. Esemplare mancante dell'estremità. - Fig. 2. Saggio assai più grande della stessa specie cui manea la parte inferiore. - Fig. 3. Disegno a contorno di un pezzo di fronda. - Fig. 4. Parte inferiore di altro esemplare. - Fig. 5. Danaeites Brongniartiana ZiGxo, pag. 209. - Fig. 6. Porzione di fronda segriata a contorno per far vedere le nervature. 


\section{IN DICE}

Cenki preliminari . . . . . pag. III-XVI

\section{ACOTYLEDONES}

Classis I. Fungi. . . . . . pag. 1

Ordo I. Iyphomycetes. . . . . . . » 2

Gen. I. Rhizomorphites Göpp. . . . . » ivi

1. Rhizomorphites intertextus Trevis. . „ivi

Ordo II. Gasteromycetes. . . . . . » 3

Gen. II. Xylomites Unger. . . . . . » ivi

*1. Xylomites irregularis Göpp. . . . » ivi

Classis II. Igae. . . . . . » ivi

Ordo III. Confervaceac. . . . . . » 6

Gen. III. Confervites Brong. . . . . » ivi

1. Confervites Veronensis Zigno. . . . »ivi

Gen. IV. Excoelocladium Zigno. . . . » 7

1. Encoelocladium tortuosum Zigno. . „ivi

Ordo IV. Phyceac. . . . . . . . . \ 8

Gen. V. Codites Sternt. . . . . . . »ivi

1. Codites serpentinus Stemb. . . . »ivi

2. \ crassipes Sternb. . . . . " 9

3. „ Krantzianus Zigno . . . . »10

Gen. VI. Encoelites Sternb. . . . . 》 ivi

1. Encoelites Mertensii Stemb. . . . »11

Ordo V. Foridene. . . . . . . . . » ivi

Gen. VII. Münsteria Sternb. . . . . » ivi

1. Münsteria encoelioides Bronn . . . \12

2. \ vermicularis Sternb. . . »13

3. \ lacunosa Sternb. . . . »ivi

Gen. VIII. Halymenites Stemb. . . . » 14

1. Halymenites vermiculatus Sternt. . »ivi
2. Halymenites cactiformis Sternb. - pag. 15

3. " varius Sternb. . . . » ivi

4. » subarticulatus Sternl. . »16

5. » secundus Stemb. . . »17

6. » Schnitzleinii Sternz. . »17

7. " cernuus Sternל. . . . " 18

8. » Stockesii Unger. . . » 19

9. » Goldfussii Sternb. . . »ivi

10. 》 Brongniartii Sternb. . »20

11. " concatenatus Sternb. . "21

12. 》 ramulosus Sternb. . . " ivi

Gen. IX. Mastocarrites Trevis . . . ” 22

1. Mastocarpites erucaeformis Trevis. . »ivi

Gen. X. Trevisania Zigno. . . . . 》23

1. Trevisania furcellata Zigno. . . . » ivi

Gen. XI. Chondrites Sternb. . . . . » 24

1. Chondrites laxus Sternb. . . . . » ivi

2. \ Solenites Unger. . . . »25

3. » lumbricarius Miunst. . . \27

4. " elongatus Presl. . . . »ivi

5. » Targionii Sternb. . . „ 28

6. » furcatus Sternb. . . . »30

7. » intricatus Sternb. . . . »31

Gen. XII. Sphaerococcites Sternb. . . »32

1. Sphaerococcites ligulatus Kurr. . . ” ivi

2. " arcuatus Presl. . . "ivi

3. " Meneghinii Zigno. . »33

4. » Savii Zigno. . . . »34

*5. » ? dichotomus Zigno. »35

Gen. XIII. Rhodymentes Trevis. . . . » ivi

1. Rhodymenites ciliatus Trevis . . „ ivi

(*) Le specie precedute da un'asterisco non appartengono alla Flora dell'Oolite, e furono comprese nelle prime dispense della presente Opera perchè in quel tempo da parecchi riputati geologi si riteneya, che le stratificazioni in cui erano state ritrovate, spettassero a qualche piano di quella formazione. 
Gen. XIV. Granularia Pomel. . . pag. 36 1. Granularia linearis Zigno . . . . »37 2. » repanda Pomel . . . " ivi Gen. XV. Corallinites Ung. . . . . 》38

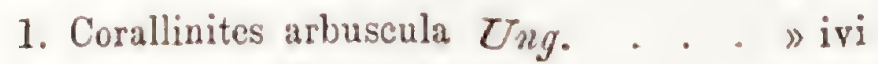

2. » Halimeda Ung. . . »39

Classis III. Calamaniac. . . . ivi Ordo VI. Calamitenc. . . . . . . . » 43

Gen. XVI. Caidanites Suck. . . . . 》 ivi

*1. Calamites Lehmannianus Göpp. . . „ ivi

*2. » Hocrensis Hiscing . . . » 44

3. » Beanii Bunbury. . . . " 45

4. " lateralis Zigno . . . „ 46

*5. „ Rogersii Bunbury . . . »48

*6. » planicostatus Rogers . . »49

Gen. XVII. Sphaenopmllum Brong. . . „50

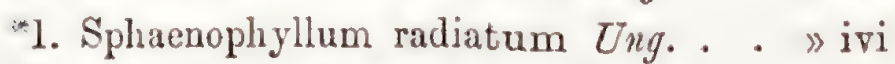

2. » australe Ung. . . »52

*3. » Trizygia Ung. . . „53

Gen. XVIII. Phyllotheca Brong. . . „ » 54

*1. Phyllotheca australis Brong. . . . „55

*2. 》. ramosa $M^{\prime}$ Coy. . . . »57

*3. » Hookeri $M^{\prime}$ Coy . . . » 59

4. 》 Brongniartiana Zigno . »ivi

5. 》 equisetiformis Zigno . . »60

Ordo VII. Equisetacenc. - . . . . » 62

Gen. XIX. Equisetites Sternb. . . . » ivi

1. Equisetites Bunburyanus Zigno . . "ivi

2. » Veronensis Zigno . . . »64

3. " columanis Sternb. . \65

Classis IV. Rilices. . . . . \68

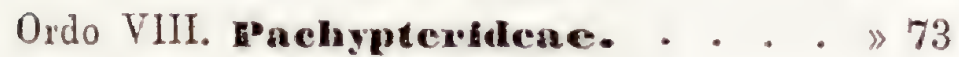

Gen. XX. Pachypteris Brong. . . . . " ivi

1. Pachypteris lanceolata Brong. . . „ivi

2. " ovata Brong. . . . . 74

3. „ Brongniartiana Zigno . »75

4. " microphylla Brong. . . „ivi

Ordo IX. Sphremopterideac. . . . „76

Gen. XXI. Sphannopteris Brong. . . . » ivi

1. Sphaenopteris denticulata Brong. . » ivi

2. \ arguta Lindl. Hutt. . »78

3. » cysteoides Lindl. Hutt. »79

4. \# athyrioides Presl. . . \80

5. 》 Münsteriana Göpp. . »81

6. » angusta Pomel. . . \82

7. „ lobophylla Pomel . „83
8. Sphaenopteris Michelinii Ponel - pag. 84 9. " pennatula Pomel . . ivi 10. " modesta Beau. . . 》 85 Gen. XXII. Hrmenophyllites Gopp. 》 86

1. Hymenophyllites macrophyllus Gopp. » 87 2. 》 crenulatus Göp. » 88 3. 》 Williamsonis Göpp.» 89 4. » Phillipsii Gopp. . » 90 5. " Murrayana Zigno. » 92 6. » Leckenbyi Zigno. » 95 7. 》 ? prisca Zigno. . » 97

Gen. XXIII. Dicroptras Pamel •. . » 98

1. Dicropteris laciniata Pomel . . . " ivi

2. 》 flabelliformis Pomel . . » 99

3. \ longifolia Pomel . . " ivi

Gen. XXIV. Loxomteris Pomel. . . » 100

1. Loxopteris elegans Pomel . . . . » ivi

Ordo X. Cyclopteridere. . . . . \ 101

Gen. XXY. Cyclopteris Brong. . . ” 102

1. Cyclopteris digitata Brong. . . . \ ivi

2. \Huttoni Stenz. . . . » 103

3. » gracilis Zigno... . » 104

4. " minor Zigno . . . »106

5. 》 incisa Eichroald . . . 》107

6. 》 lingua Lichoatd . . »108

Gen. XXVI. Odontopteris Brong. . . 》109

1. Odontopteris? jurensis Kur\%. . . » ivi

2. 》 Ungeri Źigno . . »110

3. " ? Leckenbyi Zigno . . »111

Gen. XXVII. Dichopteris Zigno . . 》 113

1. Dichopteris Visianica Zigno . . . 》 116

2. 》 lanceolata Zigno . . »118

3. „ laevigata Zigno . . . » ivi

4. » Paroliniana Zigno . . »120

5. 》 angustifolia Zigno . . »121

6. \ rhomboidalis Zigno. . »122

7. » microphylla Zigno . . » ivi

Ordo XI. vemopterideae. . . . . 》 124

Gen. XXVIII. Neunopteris Brong. . . 》 ivi

1. Neuropteris undulata Lindl. Hult. . » ivi

2. » arguta Lindl. IIutt. . " 126

Ordo XII. Pecopterideac. . . . " " 127

Gen. XXIX. Pecopteris Brong. . . . 》 ivi

1. Pecopteris recentior Phillips . . . \ivi

2. » denticulata Brong. . . » 129

3. » lobifolia Lindl. Hutt. . »131

4. 》 serrata Ung. . . . . » 132

5. » Huttoniana Presl. . . » 133 
6. Pecopteris insignis Lindl. Hutt. pag. 135

7. » Haiburnensis Lindl. Ifutt. »137

*8. » Ottonis Göpp. . . . . »138

9. » Phillipsii Brong. . . . » 139

10. 》 ligata Phillips. . . . . » 140

11. 》 Whitbiensis Brong. . • » 142

12. 》 exilis Phillips. . . . . »144

13. " acutifolia Lindl. Hutt. . »146

14. » ctenis Ponel . . . . »147

15. » Ittieri Pomel . . . . »148

16. 》 Moretiana Brong. . . . » 149

Gen. XXX. Acrostichites Göpp. . . . » ivi

1. Acrostichites Williamsonis Göpp. . 》 ivi

Gen. XXXI. Crcadopteris Zigno . . . »15l

1. Cycadopteris Brauniana Zigno . . » 155

2. 》 undulata Zigno. . . »157

3. 》 heterophylla Zigno . »158

4. 》 Heerii Zigno. . . . \160

Gen. XXXII. Polypodites Göpp. . . . \161

1. Polypodites Lindleyi Göpp. . . . \162

2. „ crenifolius Göpp. . . »153

3. » undans Göpp. . . . »164

Gen. XXXIII. Phyllopteris Brong. . . 》 166

1. Phyllopteris Phillipsii Brong. . . „ ivi

Gen. XXXIV. Marzaria Zigro. • . • »168

1. Marzaria Paroliniana Zigno . . . »170

Ordo XIII. Dictyopteridene. . . . "171

Gen. XXXY. Phlebopteris Brong. . . „172

1. Phlebopteris polypodioides Brong. . " ivi

2. " contigua Lindl. IIult. " 173

3. 》 Woodwardii Leckenby. » 174

Gen. XXXVI. Dictrophyllum Lindl. Hutt. » 176

1. Dictyophyllum rugosum Lindl. Hutt. » ivi

2. 》 Leckenbyi Zigno. . „178

Gen. XXXVII. Protorhipis Andrae . . 180

1. Protorhipis Asarifolia Zigno . . . ” ivi

Gen. XXXVIII. Camptopteris Presl. . „ 181

1. Camptopteris jurassica Göpp. : . » ivl

Gen. XXXIX. SAGenopteris Presl. . . ” 182

1. Sagenopteris cuneata Morris . . „183
2. Sagenopteris reniformis Zigno. - pag. 184

3. 》 angustifolia Zigno . \186

4. » Phillipsii Presl. . . »187

5. 》 Goeppertiana Zigno - 》188

Gen. XL. Ctenss Lindl. Hutt. . . . . »190

1. Ctenis falcata Lindl. Hutt. . . . " ivi

Ordo XIV. Gleicheniaceac. . . . . »192

Gen. XLI. Gleichenites Göpp. . . . » ivi

1. Gleichenites elegans Zigno. . . . » 193

2. 》 ? Desnoyersii Zigno. . „ 194

Gen. XLII. Laccopteris Presl . . . . „195

1. Laccopteris Phillipsii Zigno . . . „ ivi

2. 》 Rotzana Zigno. . . . »197

Ordo XV. garattinceac. . . . . „199

Gen. XLIII. Taeniopteris Brong. . . » 199

1. Taeniopteris vittata Brong. . . . \201

2. " latifolia Brong. . . . \204

3. » Williamsonis Göpp. . »205

4. " ovalis Prest . . . \206

Ordo XVI. Danneaceac. . . . . . »207

Gen. XLIV. Danaeites Göpp. . . . » ivi

1. Danaeites Heerii Zigno. . . . . 》208

2. " Brongniartiann Zigno. . „209

Ordo XVII. Schixcaceac. . . . . . »210

Gen. XLV. Stachypteris Pomel . . ” ivi

1. Stachypteris spicans Pomel. . . . \211

2. \ litophylla Pomel. . . 》 ivi

3. \ pulchra Pomel . . \212

Classis V. Lycopochaceac. " ivi

Ordo XVIII. Eycopodicac. . . . . 》213

Gen. XLVI. Lxcopodites Brong. . . . " ivi

1. Lycopodites falcatus Lindl. Hutt. . " ivi

Ordo XIX. psinotenc. . . . . . . »214

Gen. XLVII. Psicotres Minst. . . . " ivi

1. Psilotites filiformis Minst. . . . \ ivi

Gen. XLVIII. Isoetrtes Göpp. . . . . »215

1. Isoctites crociformis Minst. . . . » ivi

2. » Murrayana Unger. . . \216 


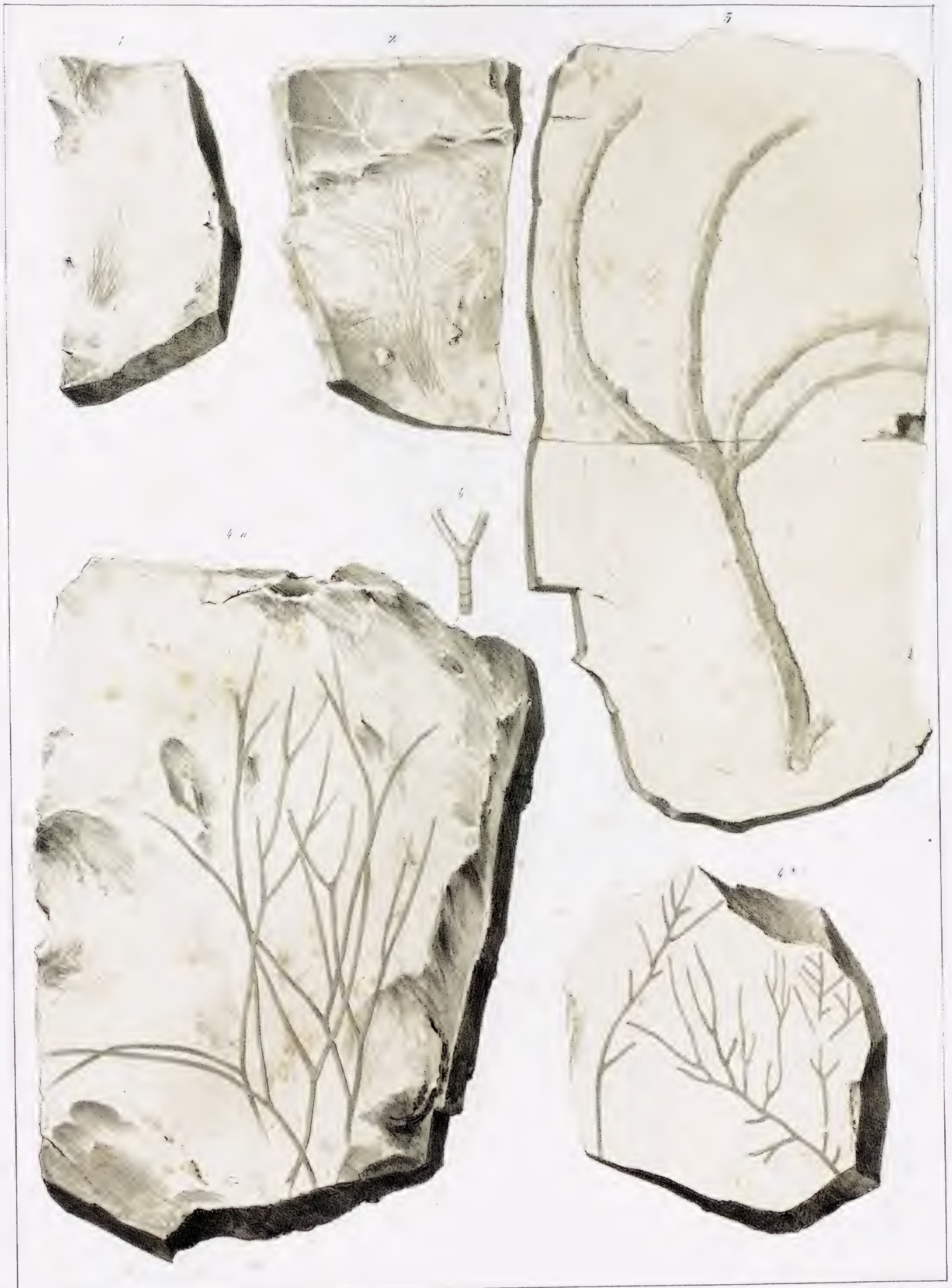



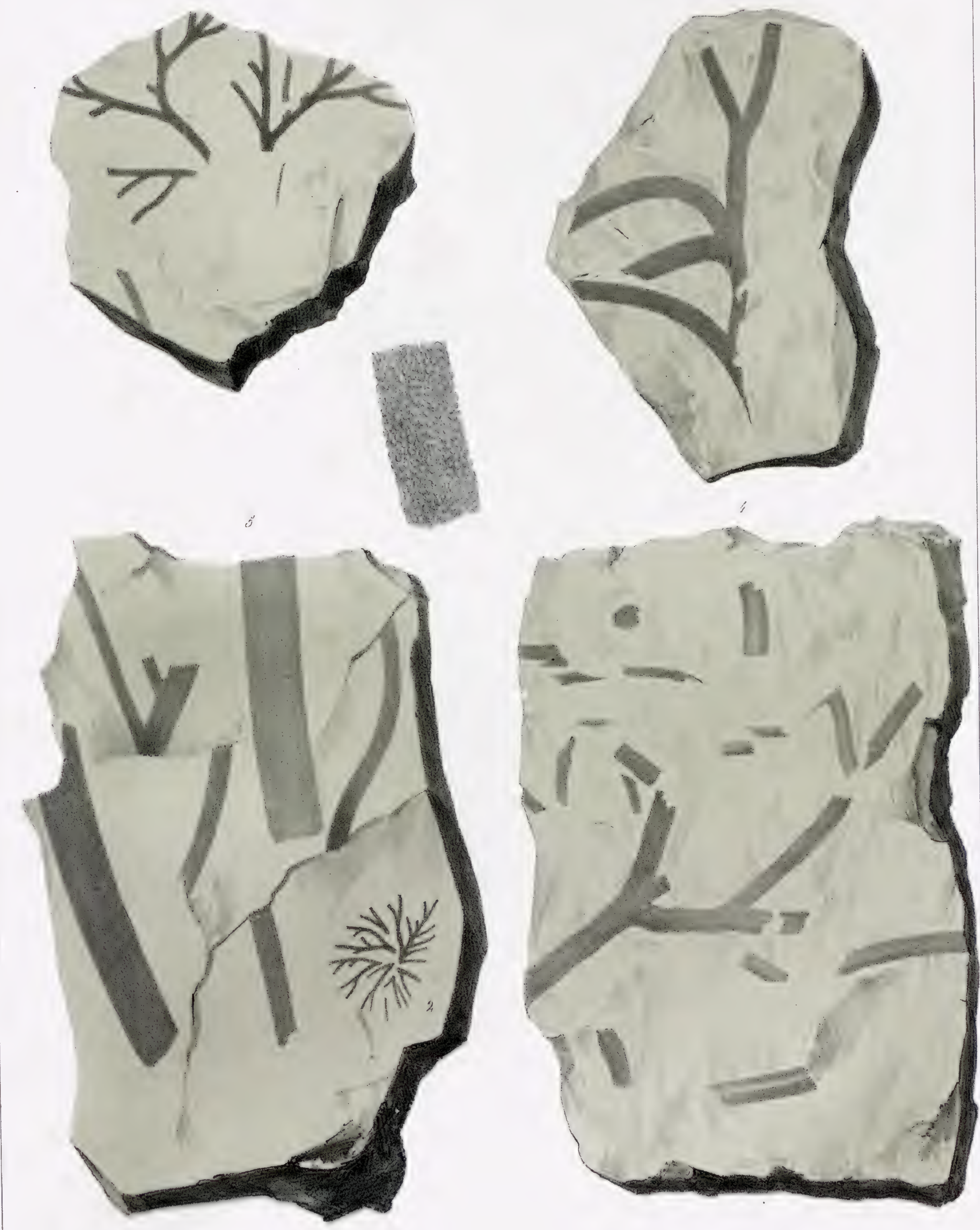

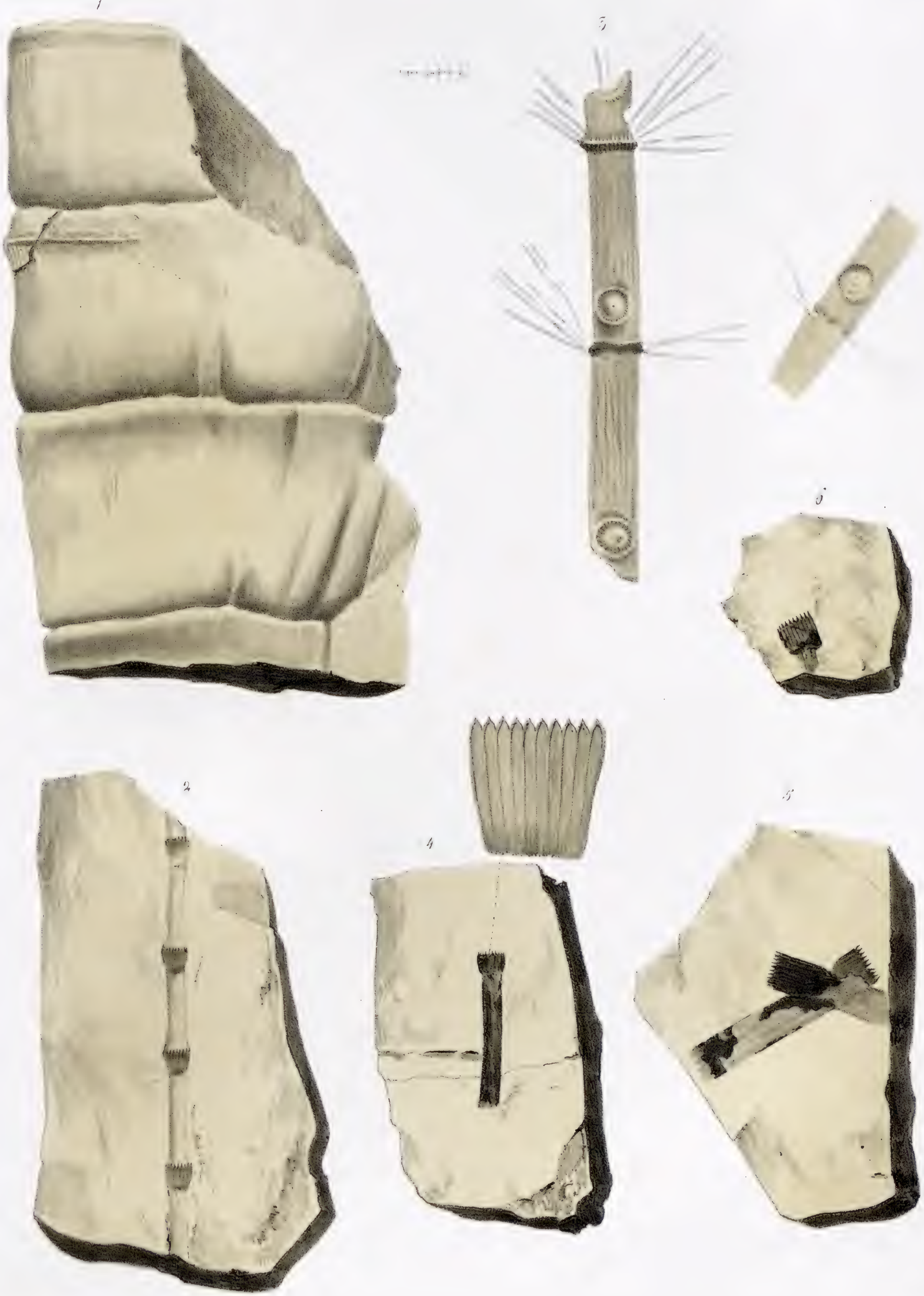


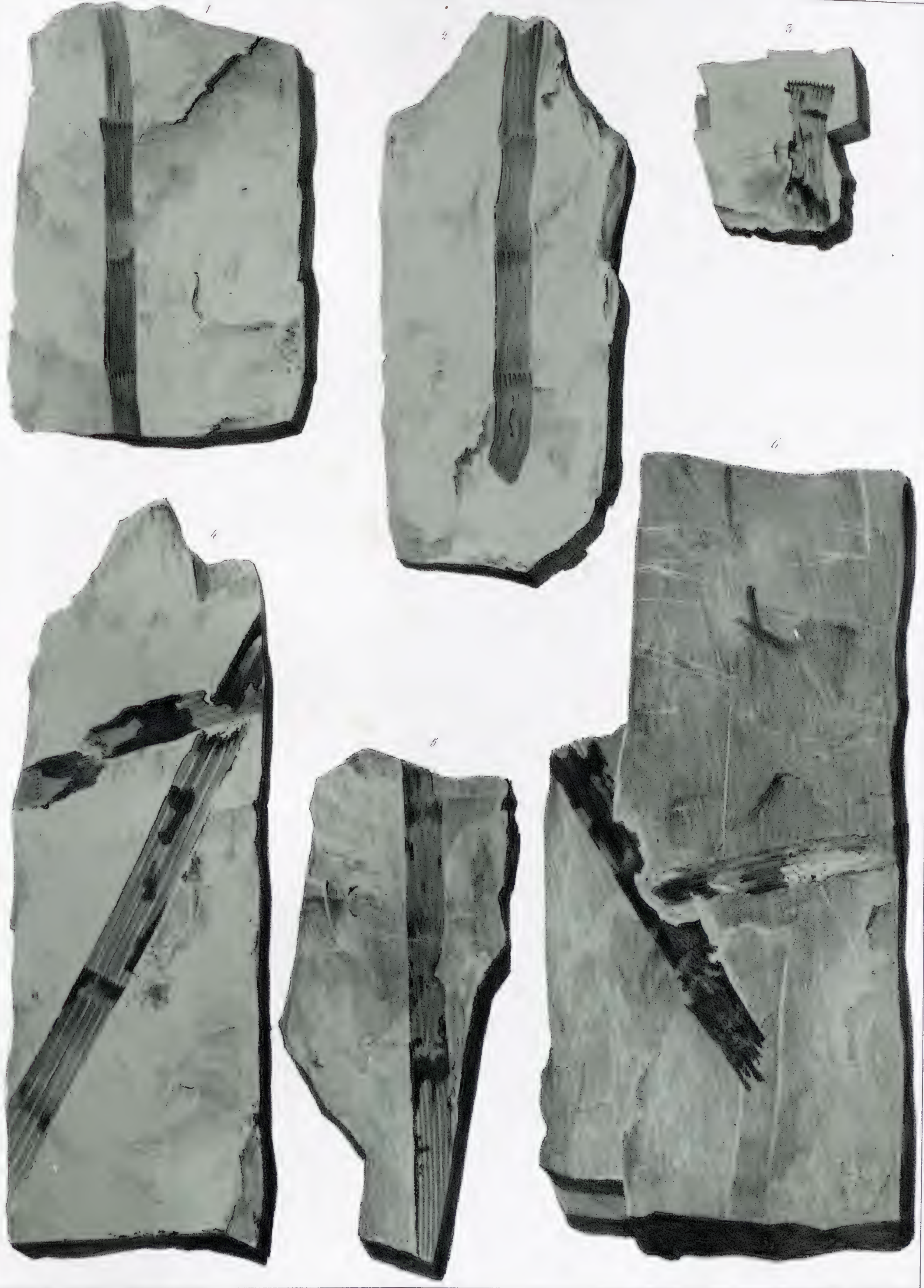



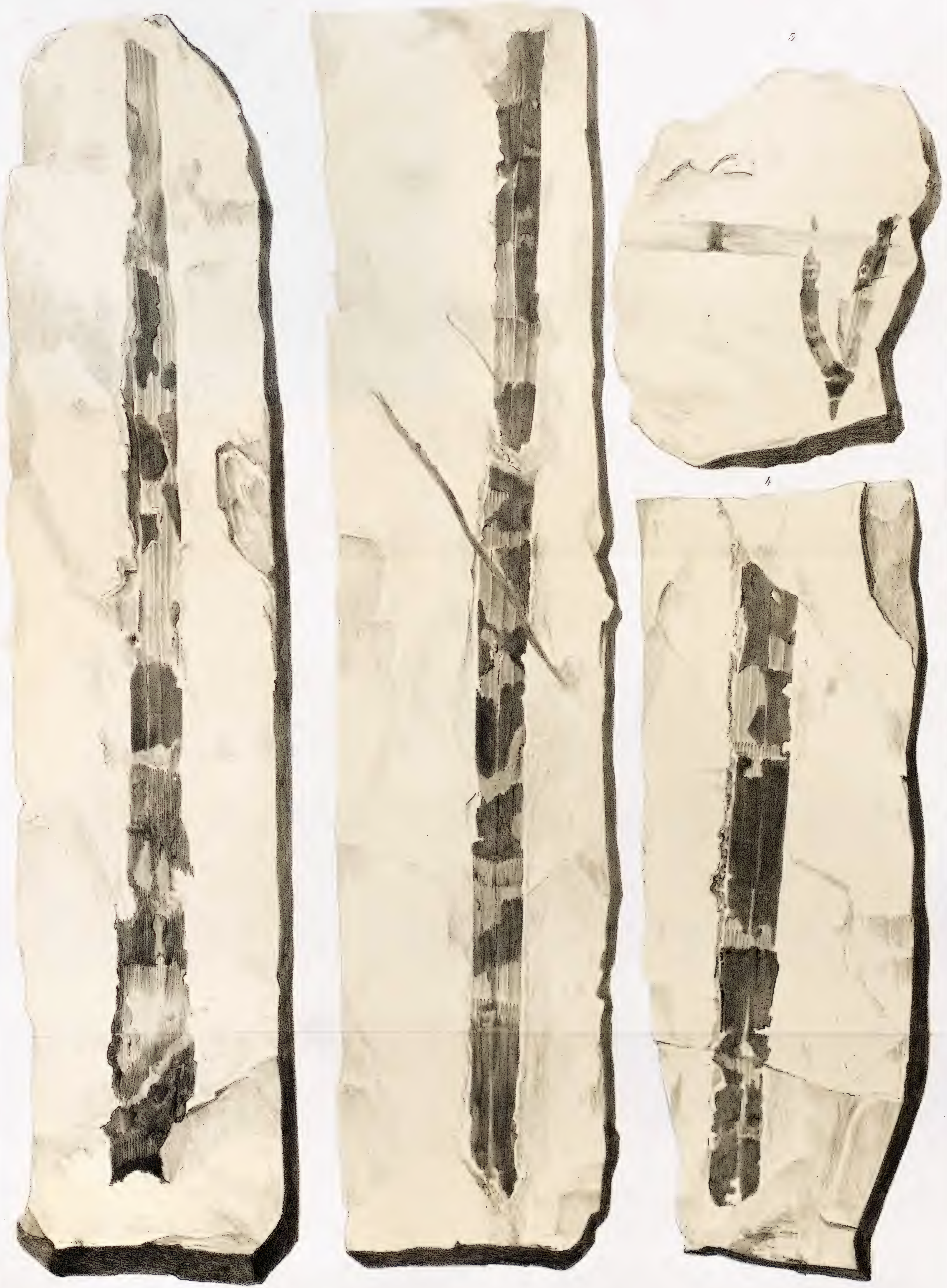


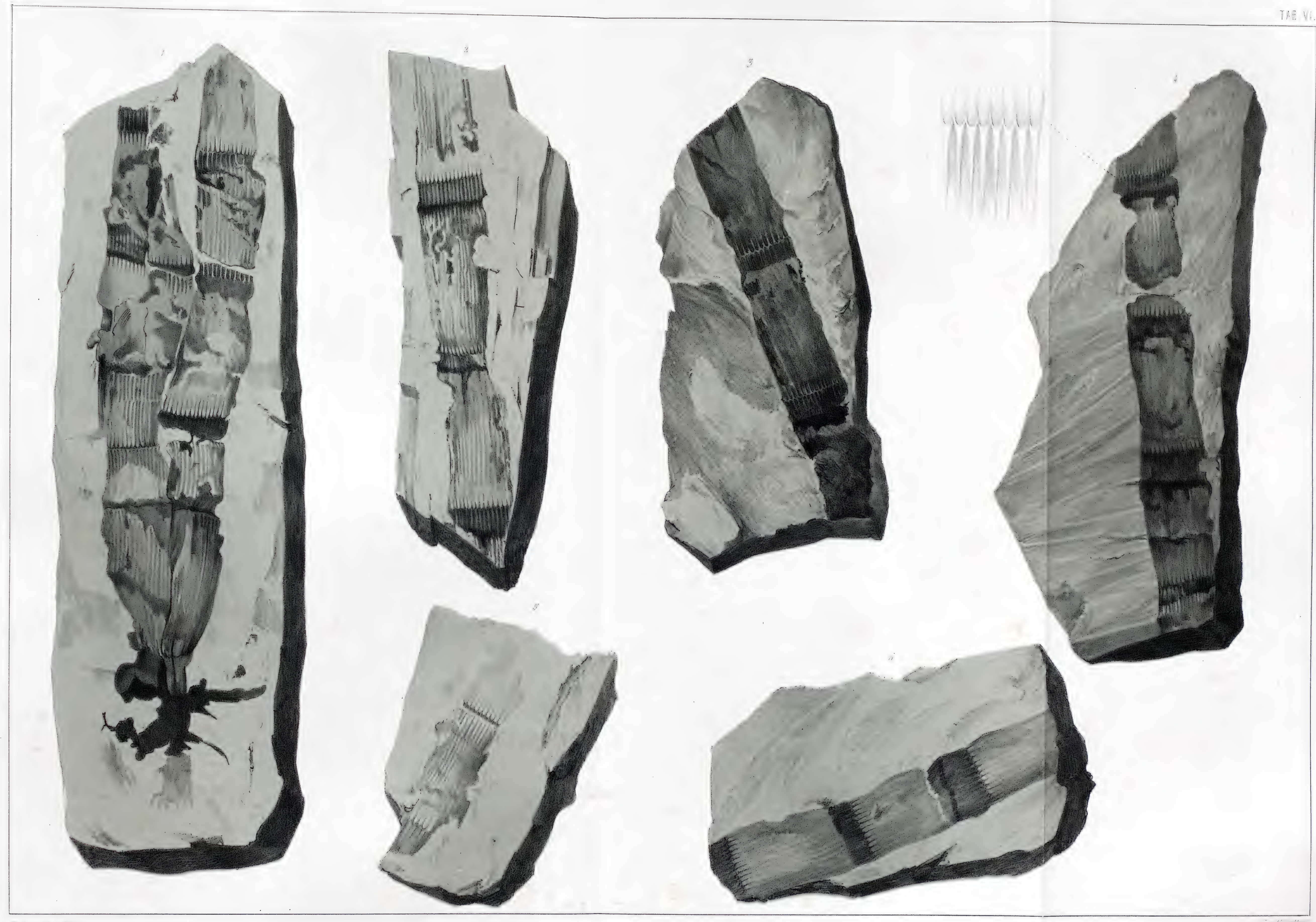




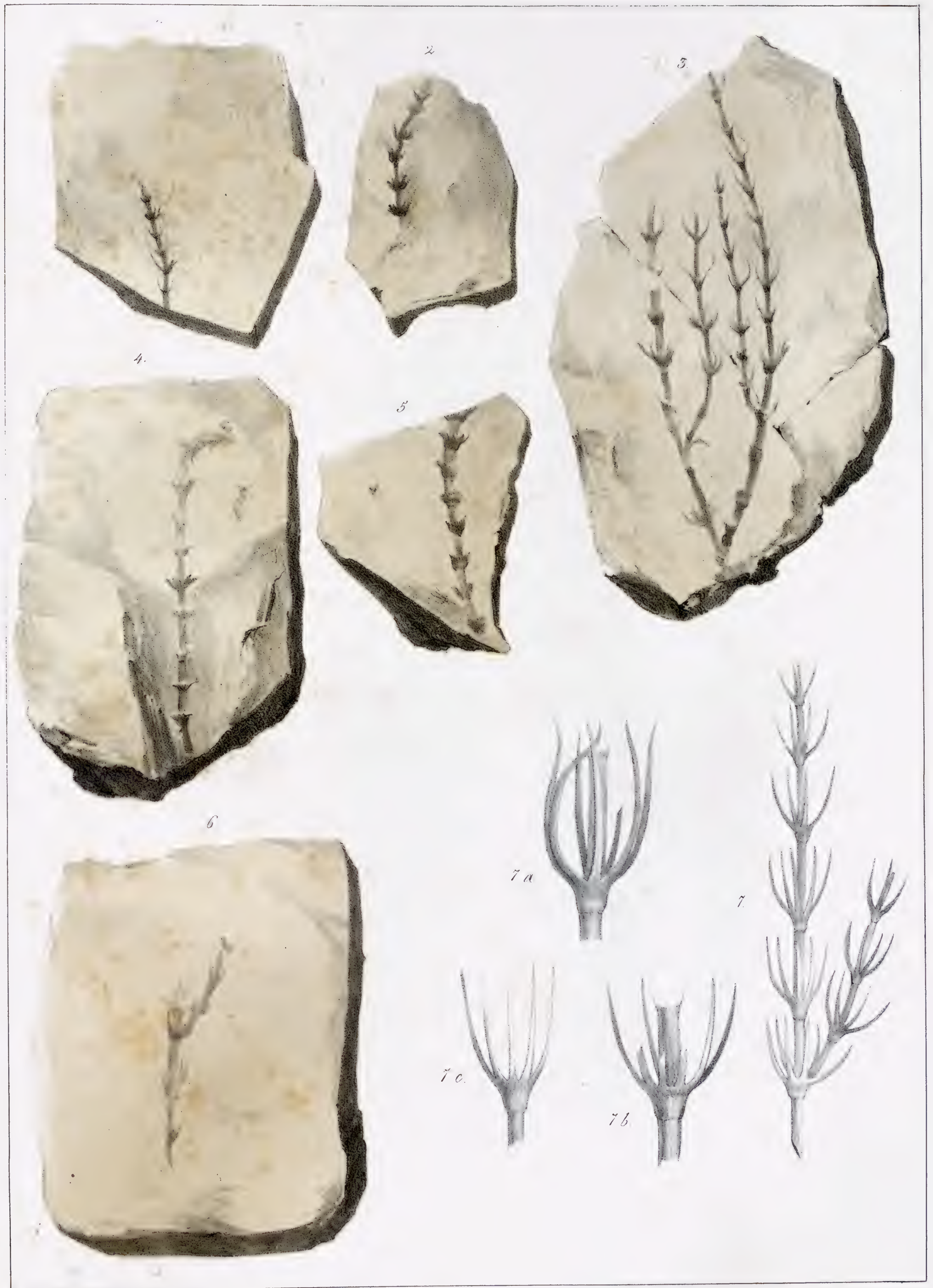




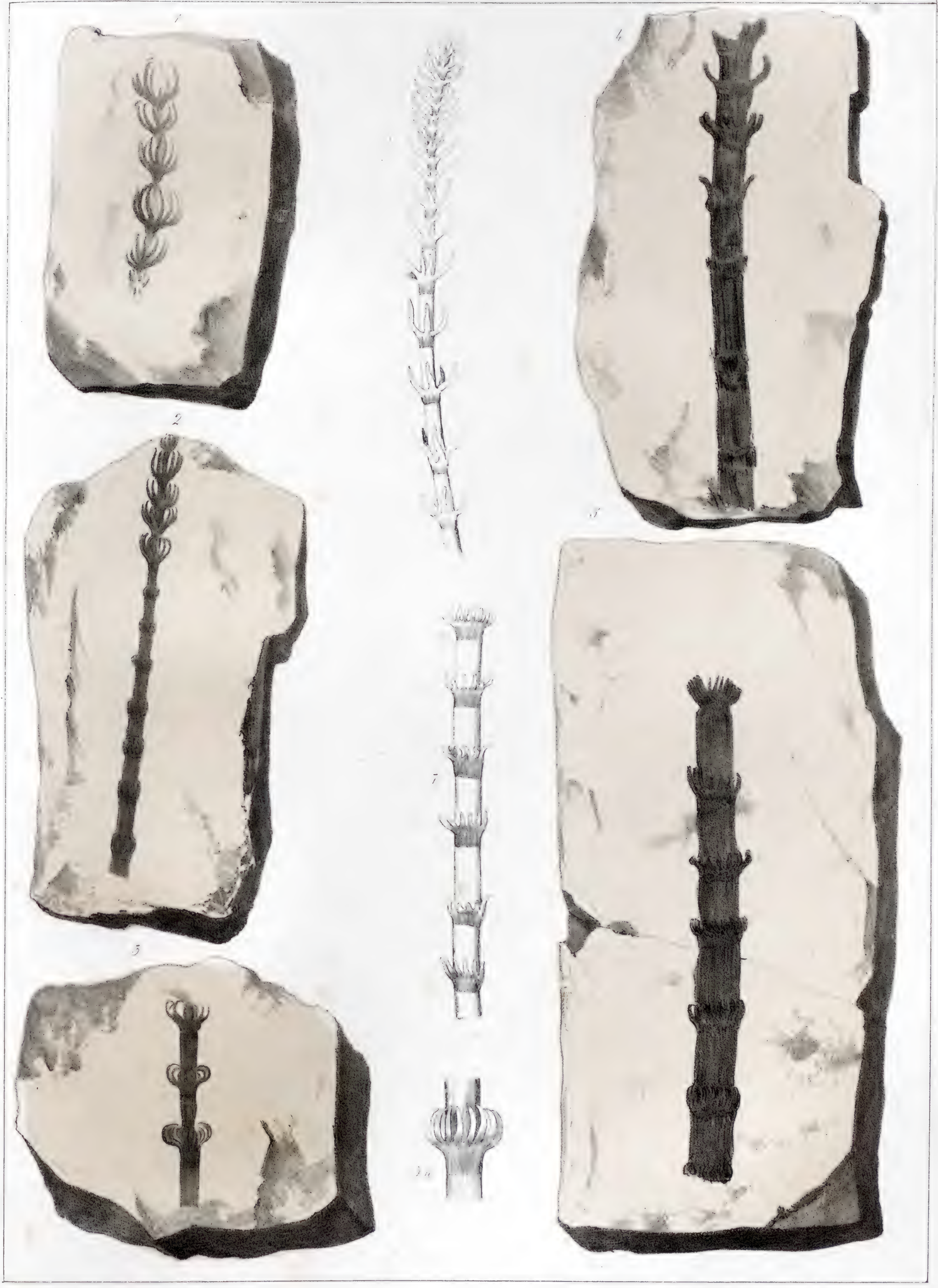


TAB. IX

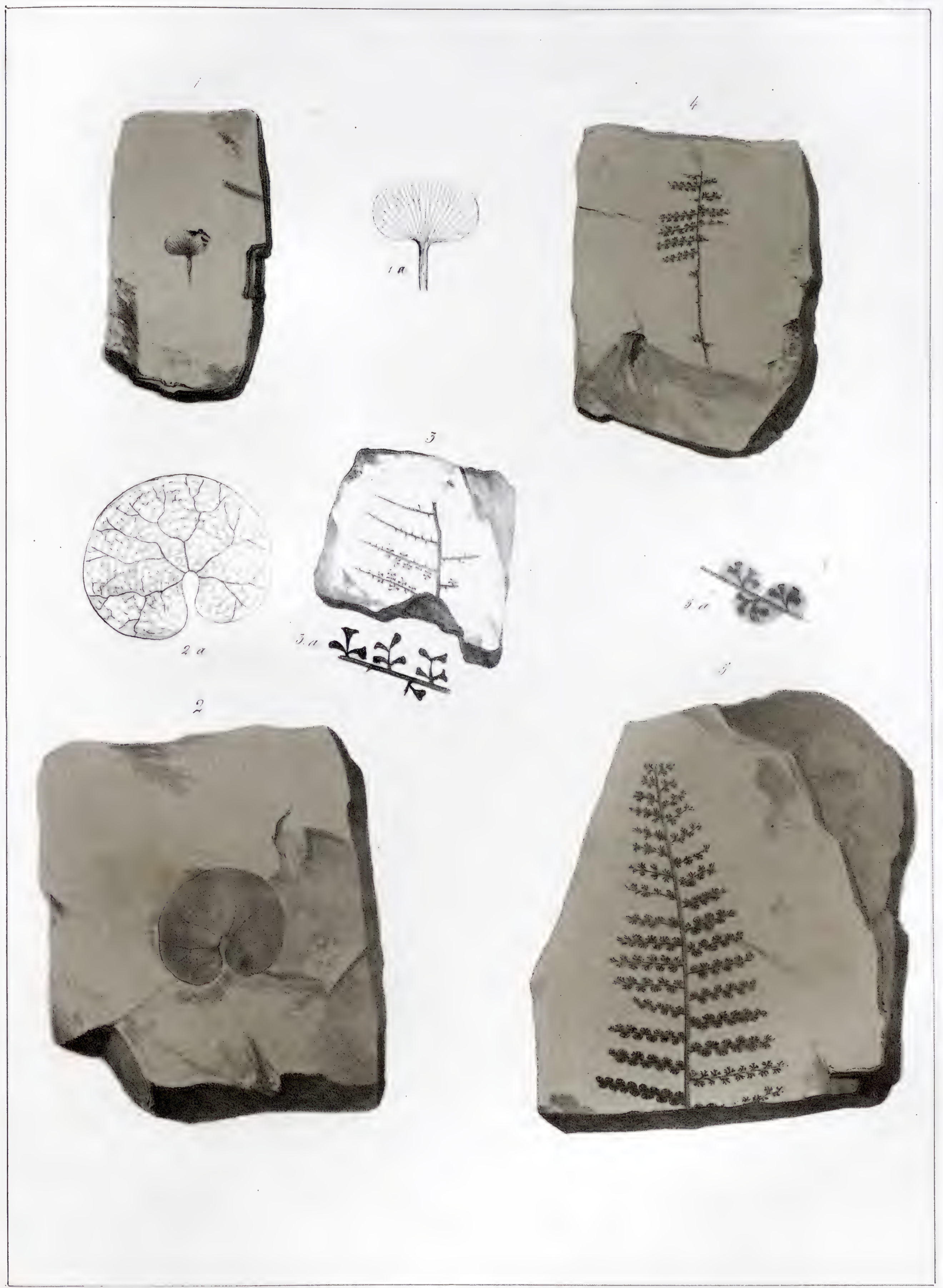


TAB $X$.

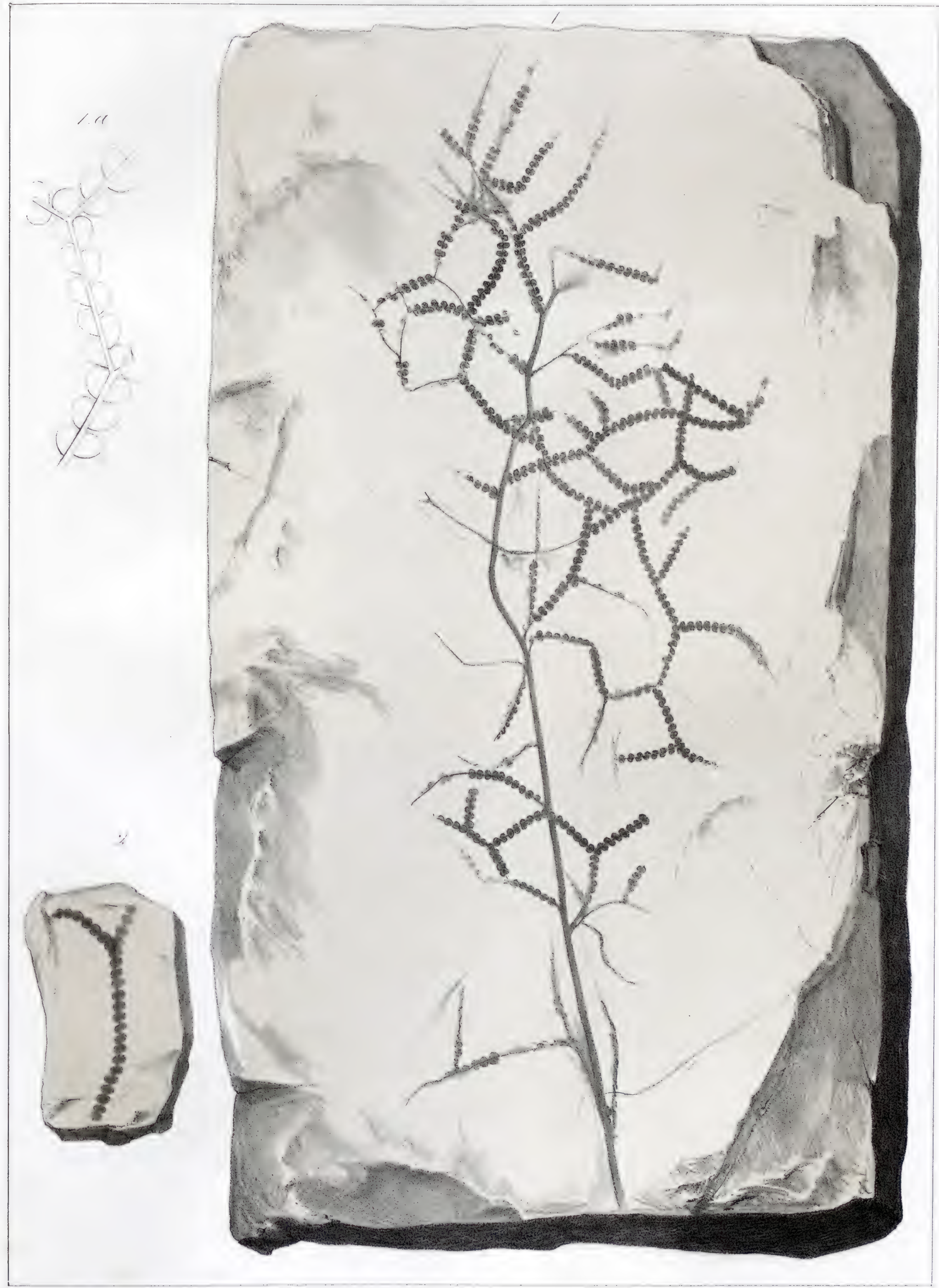




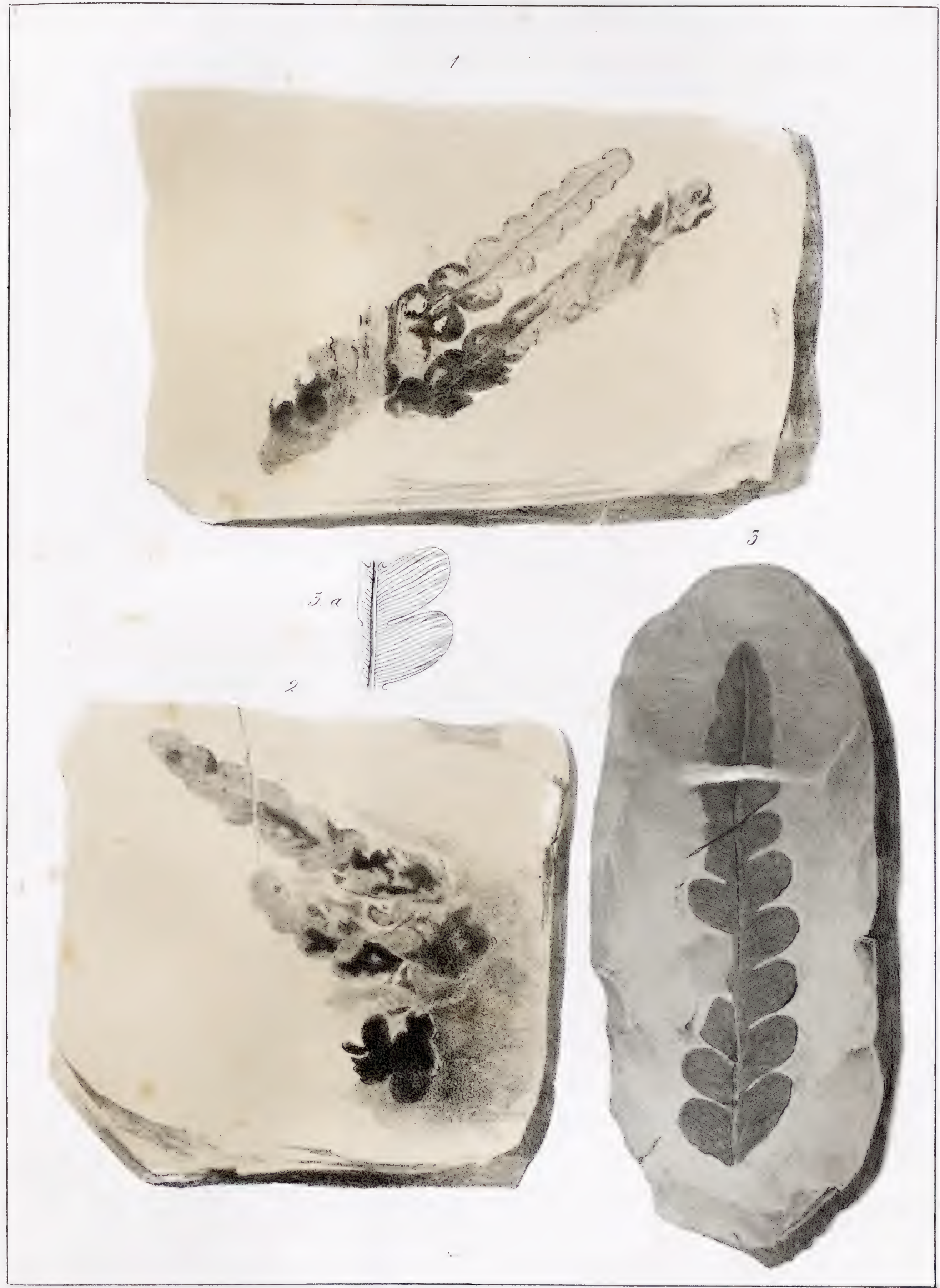



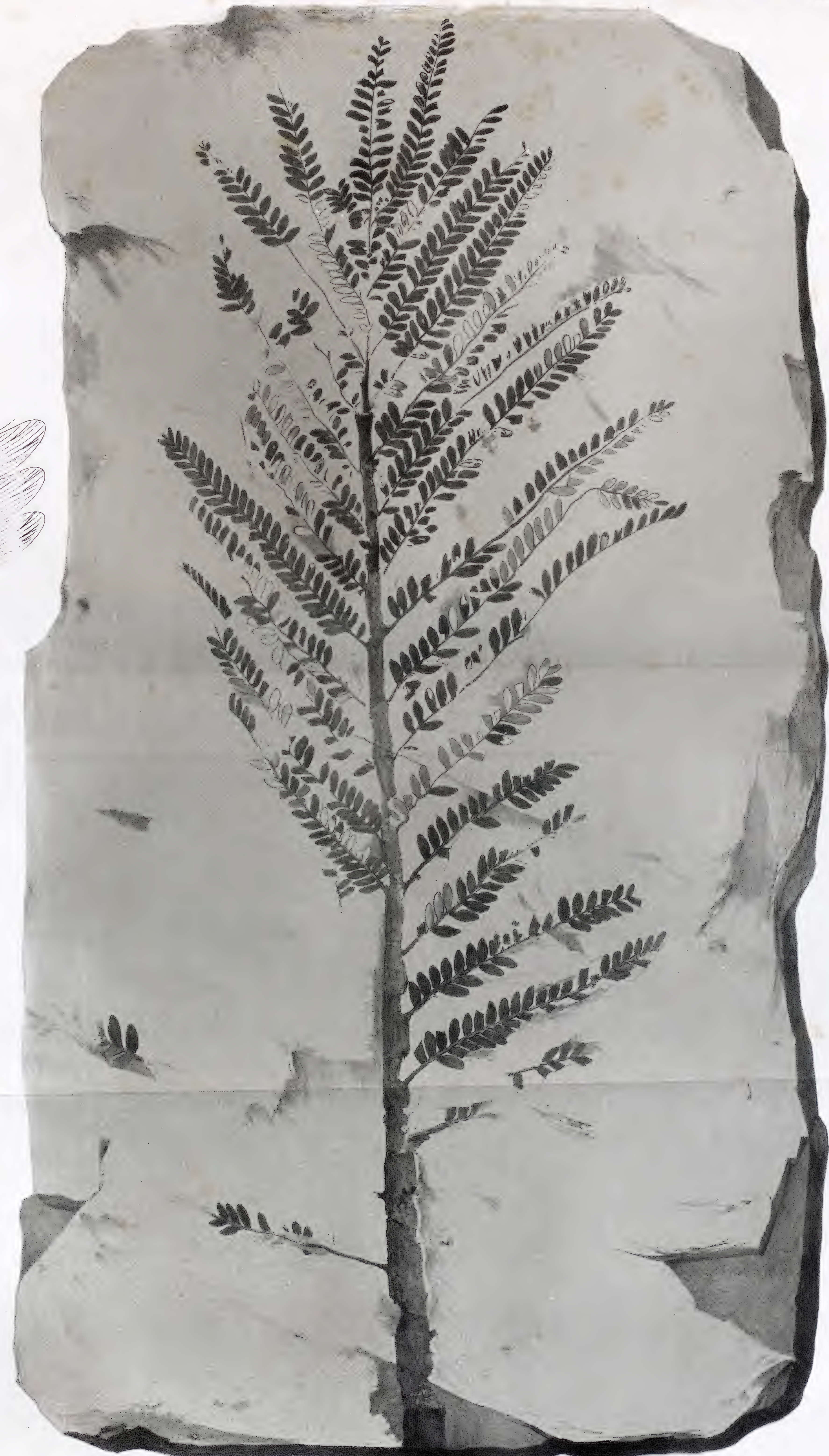


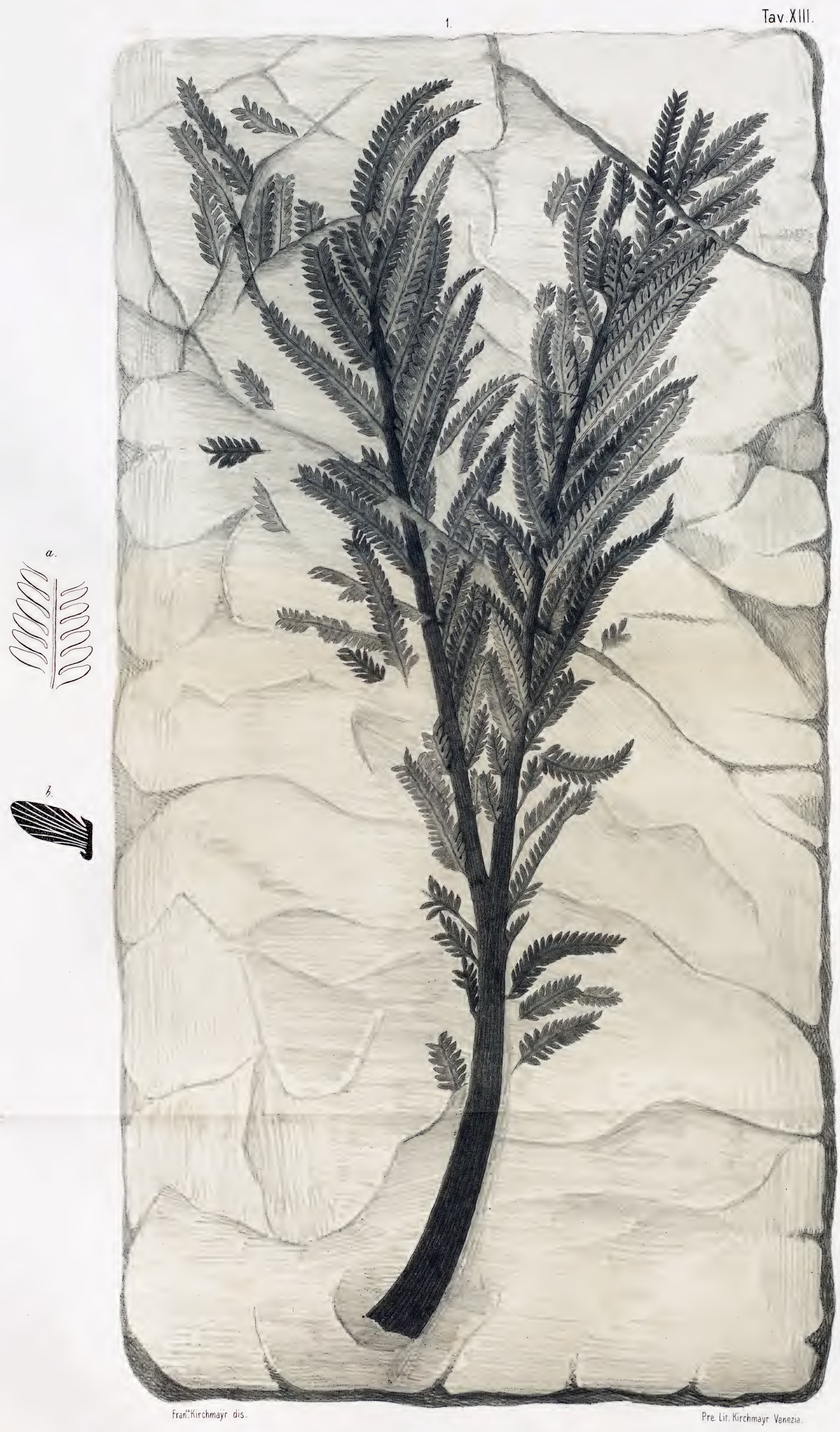



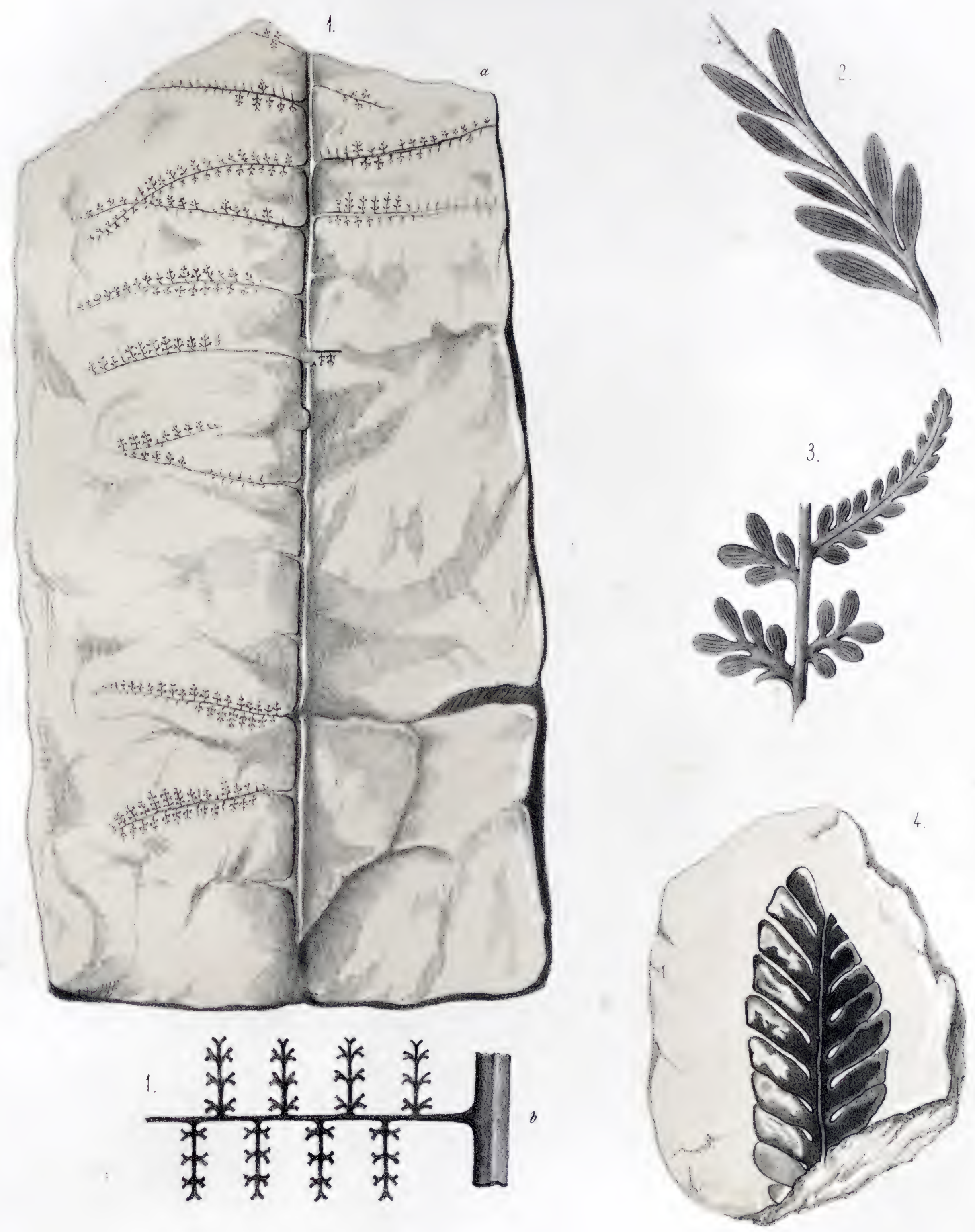

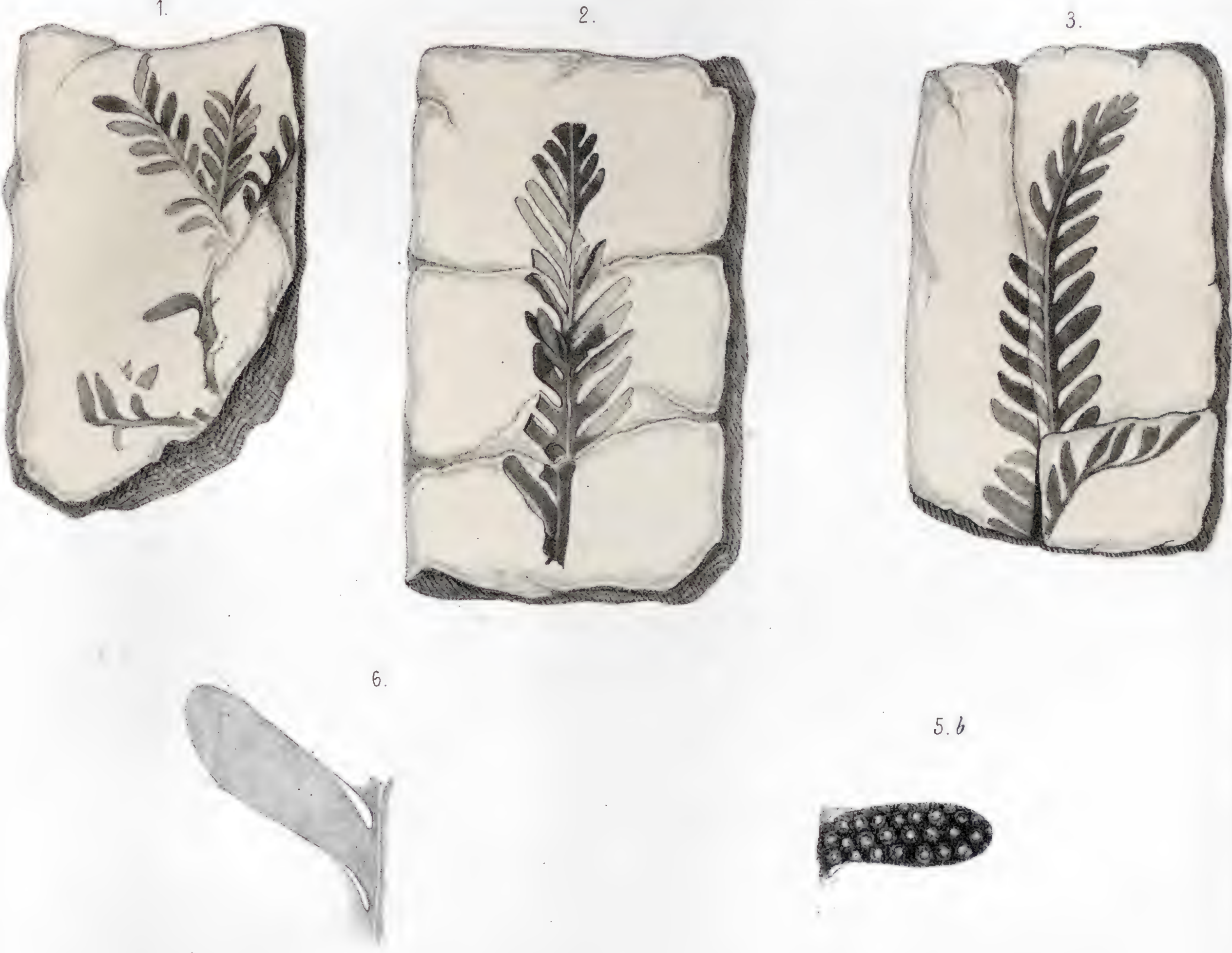

5.6
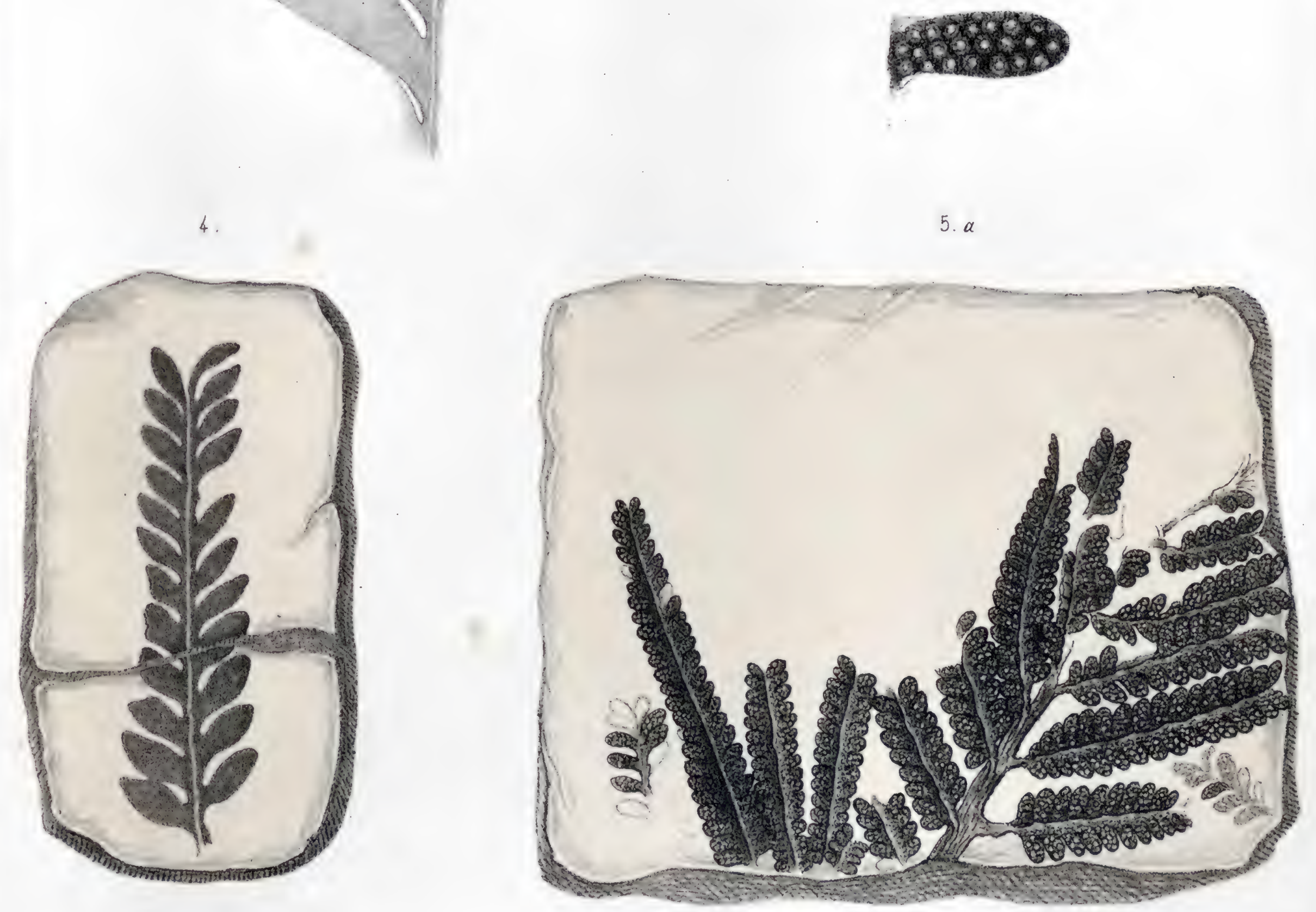

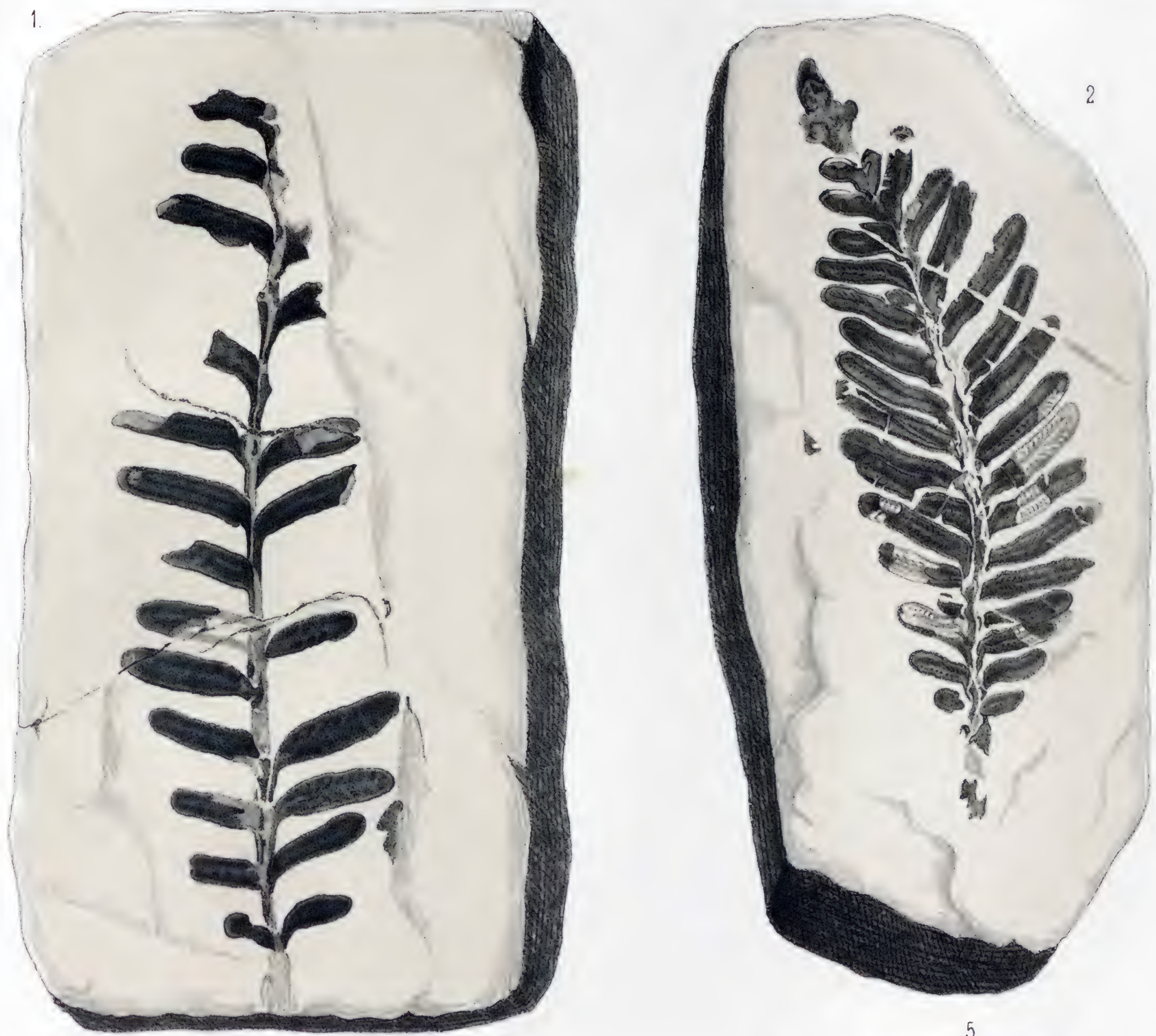

3.
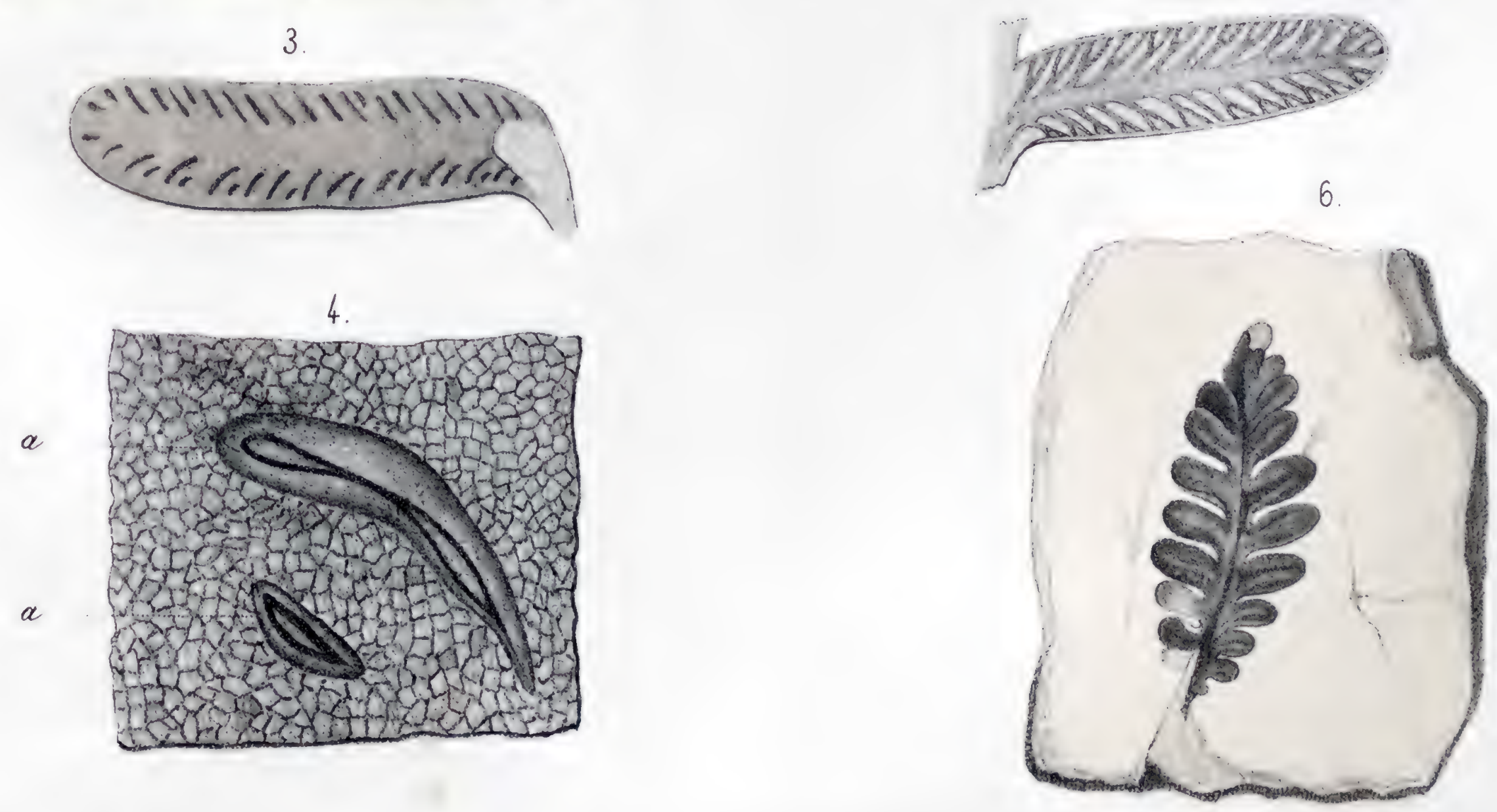

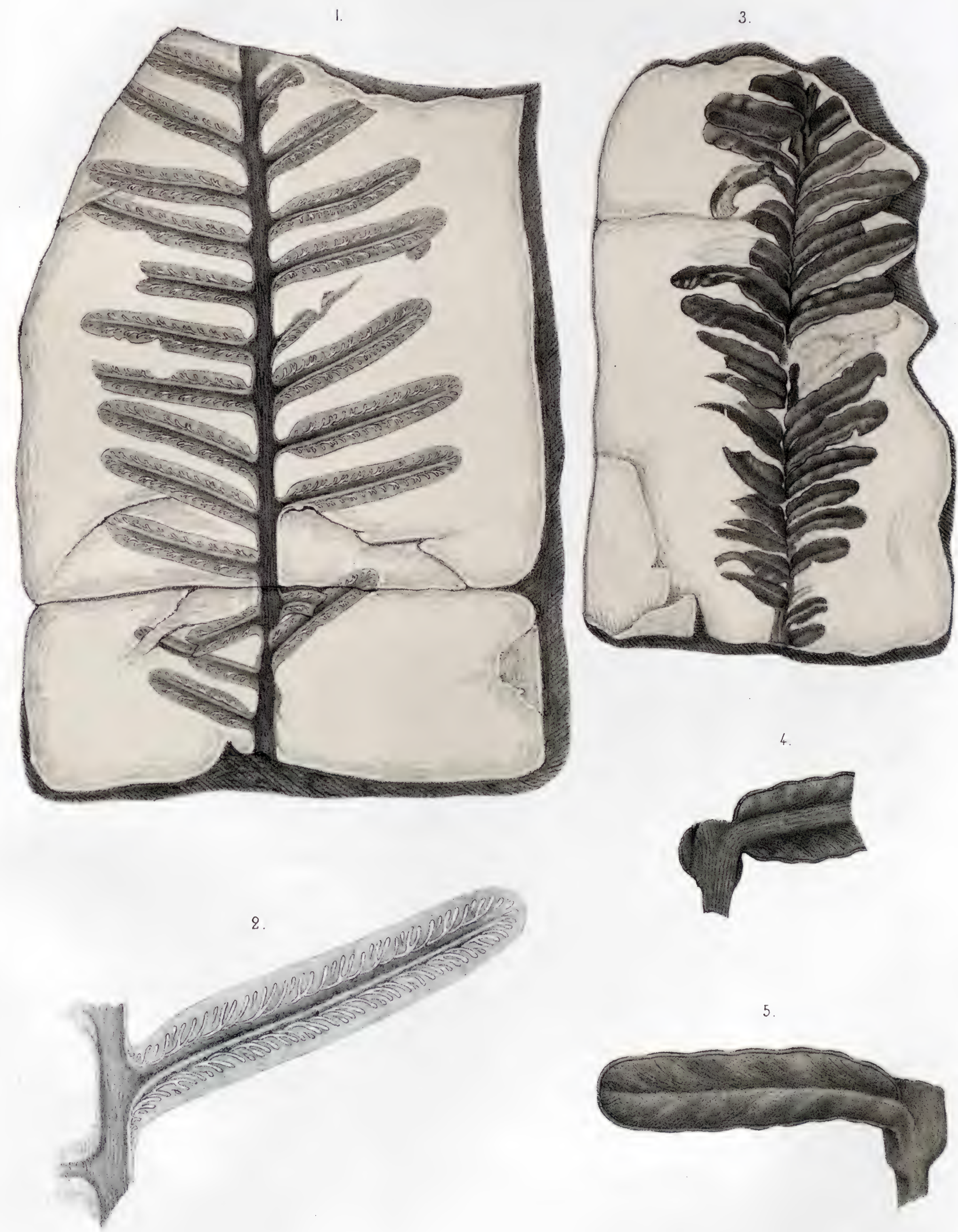

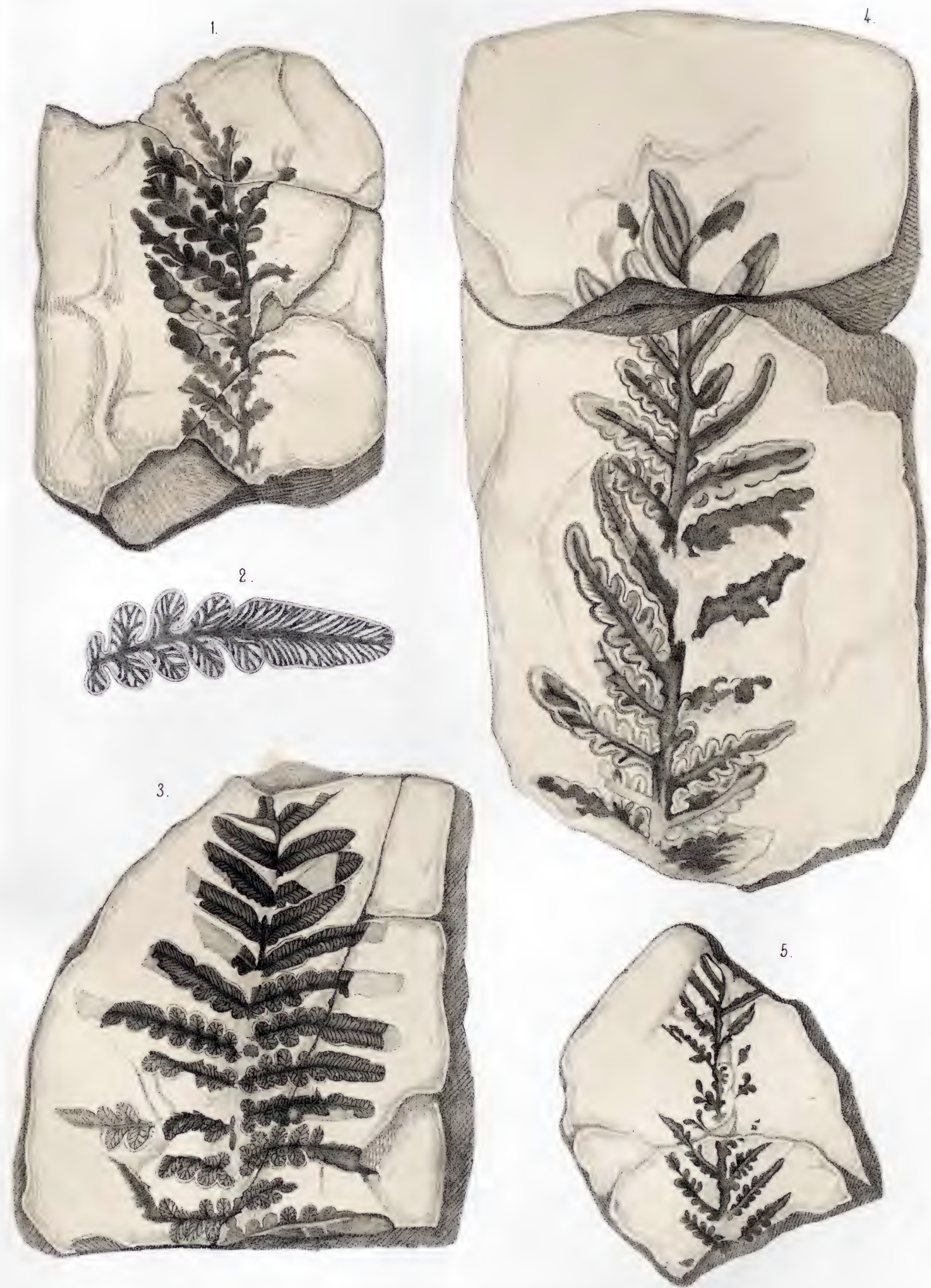


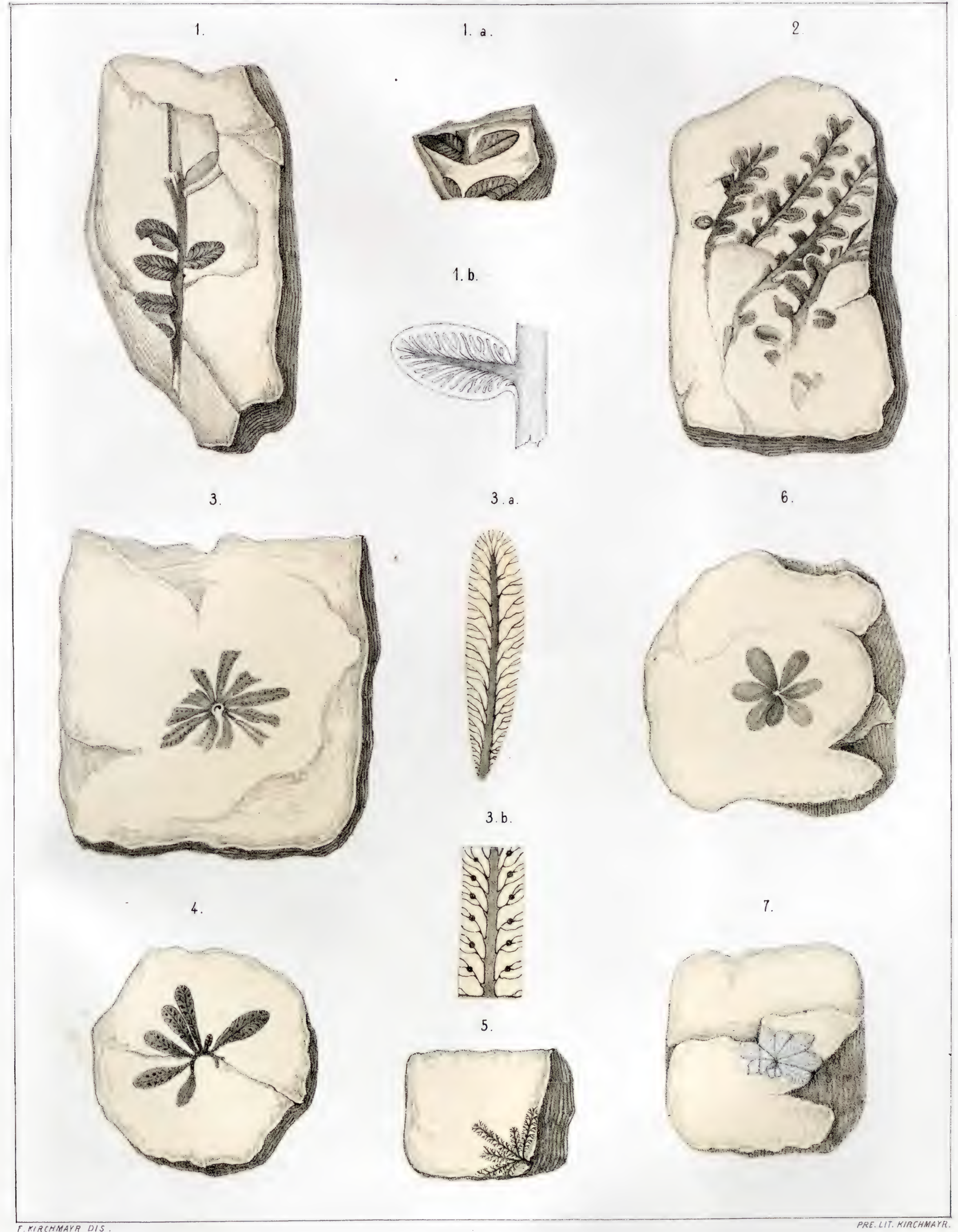




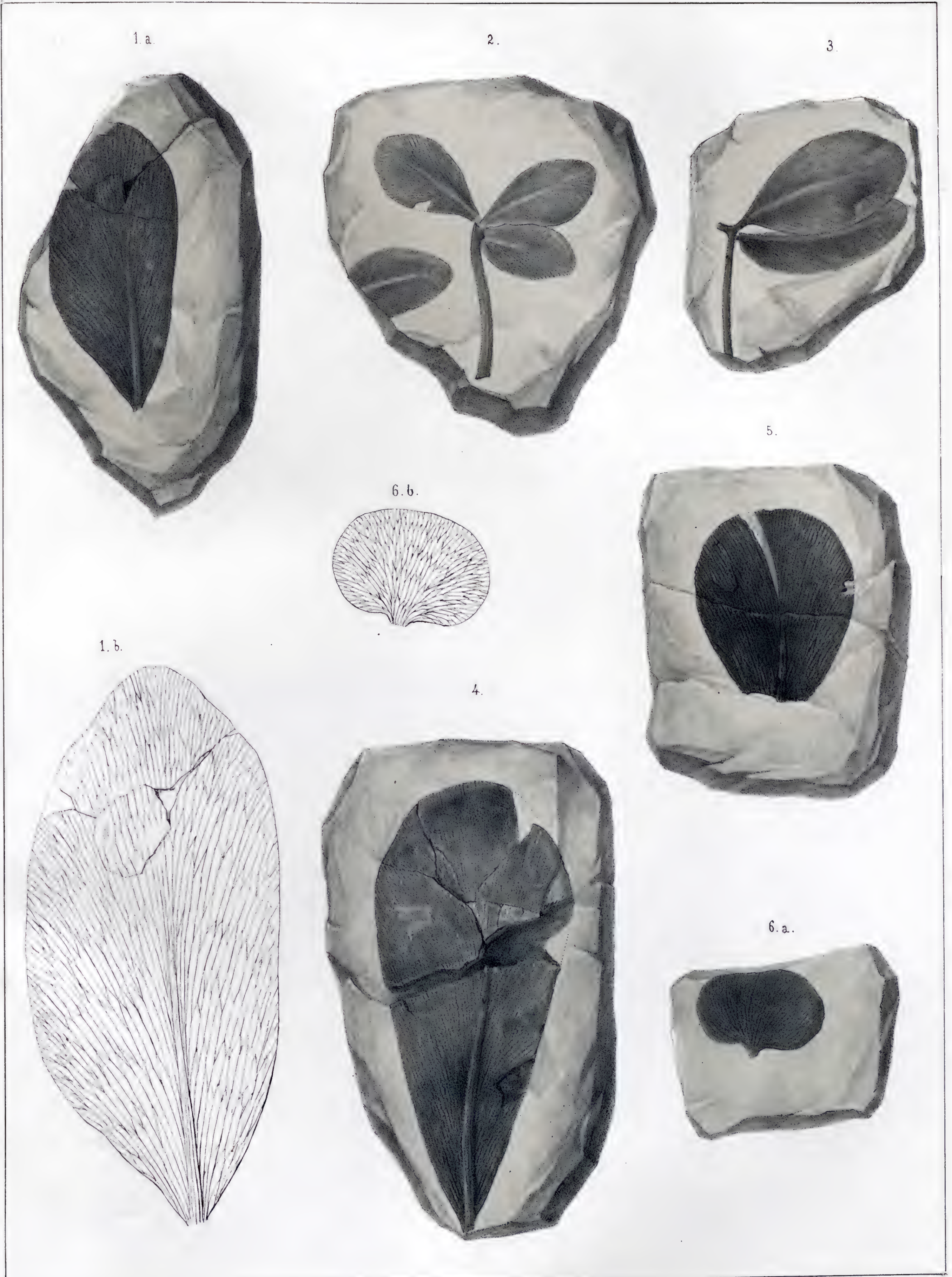



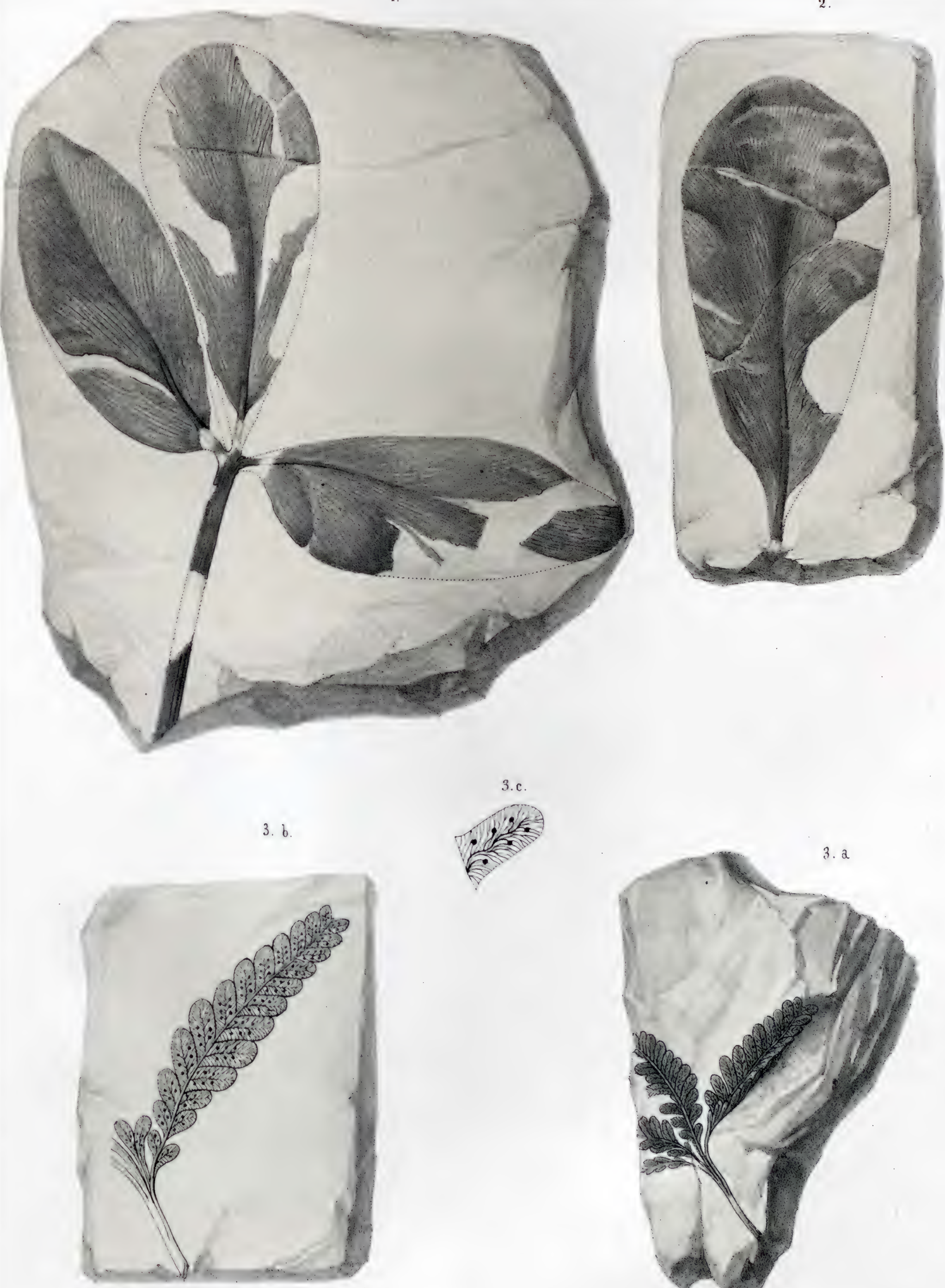

3.c.

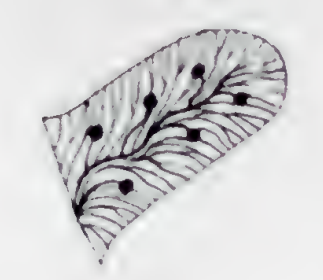




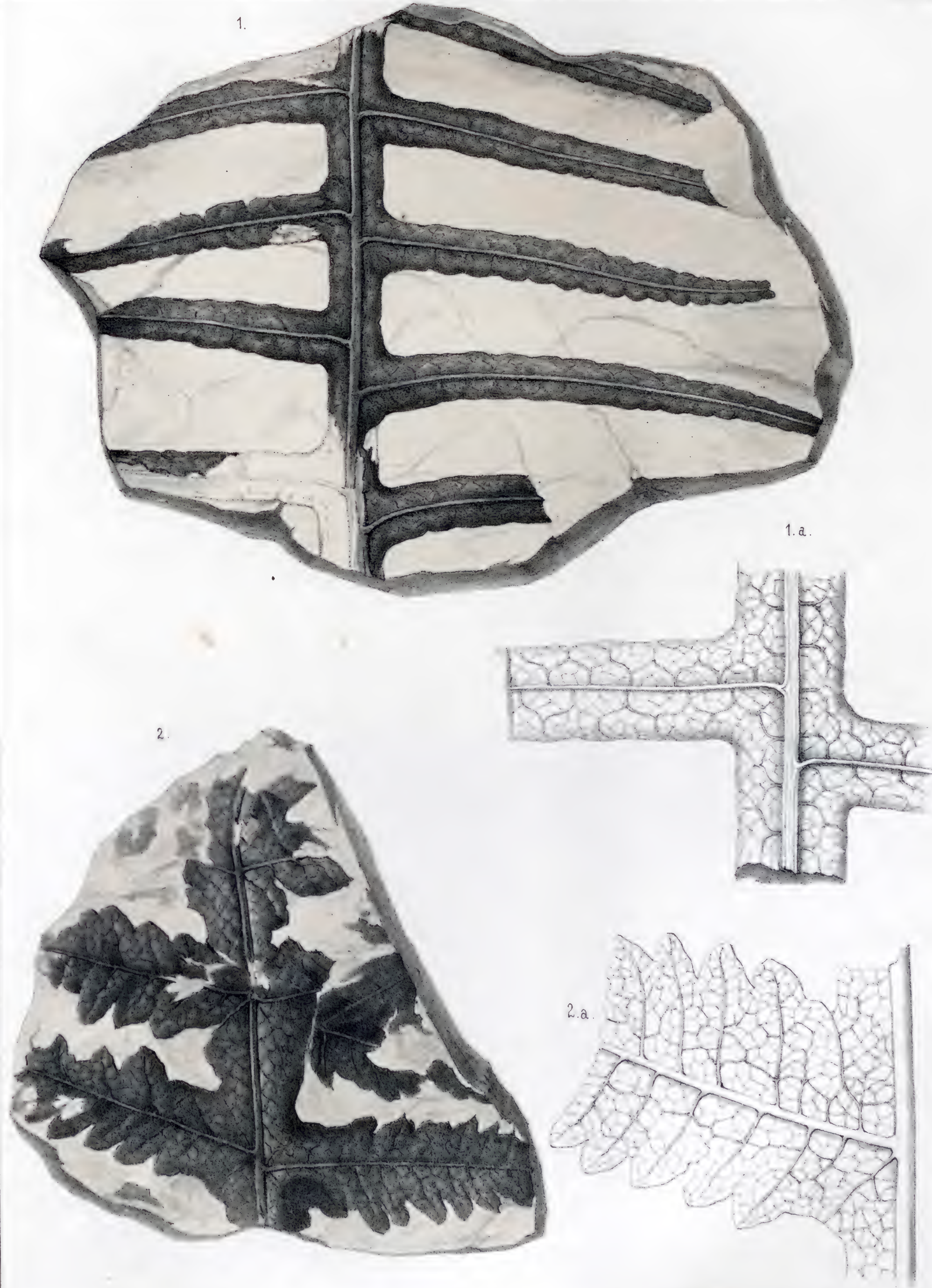



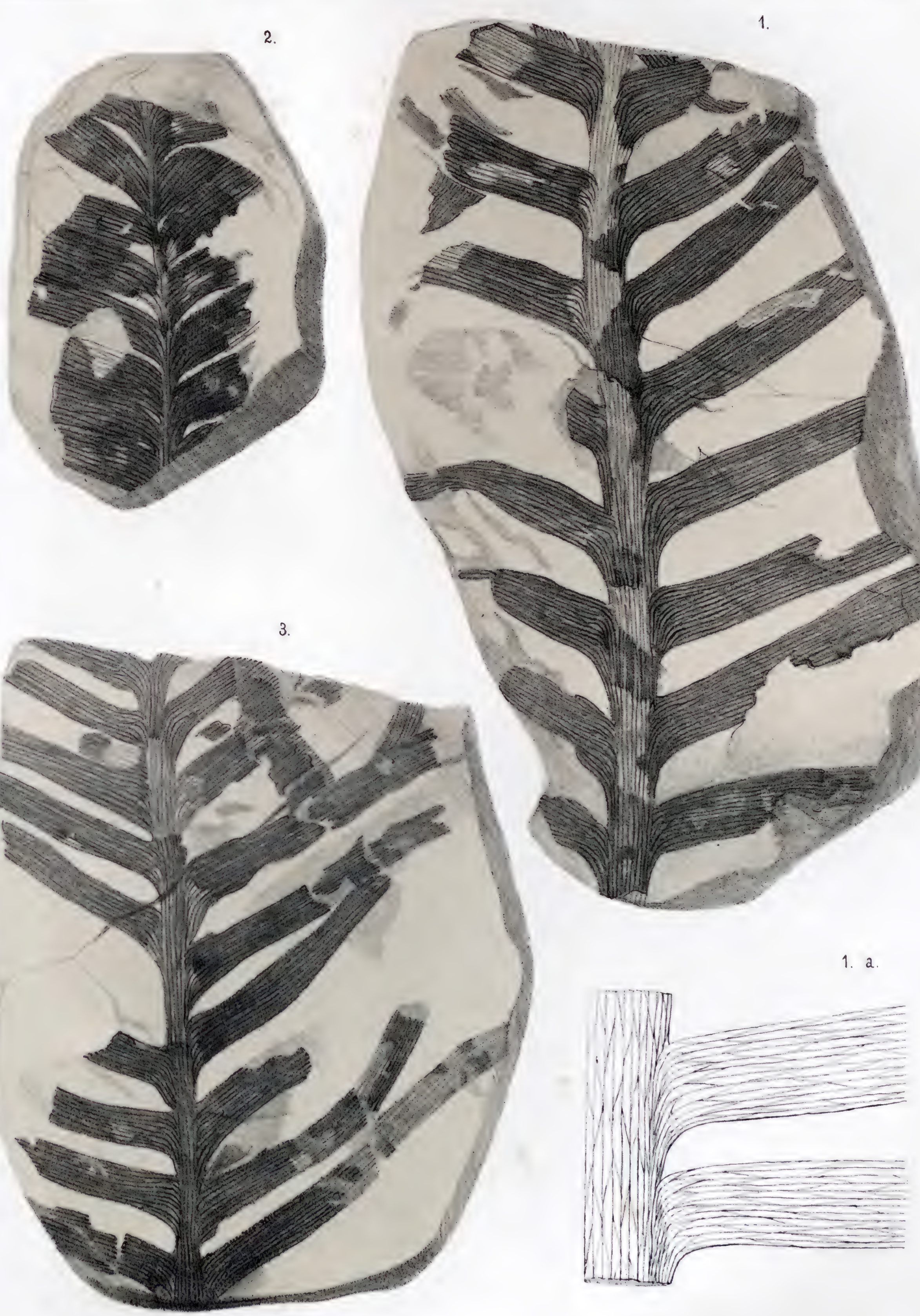


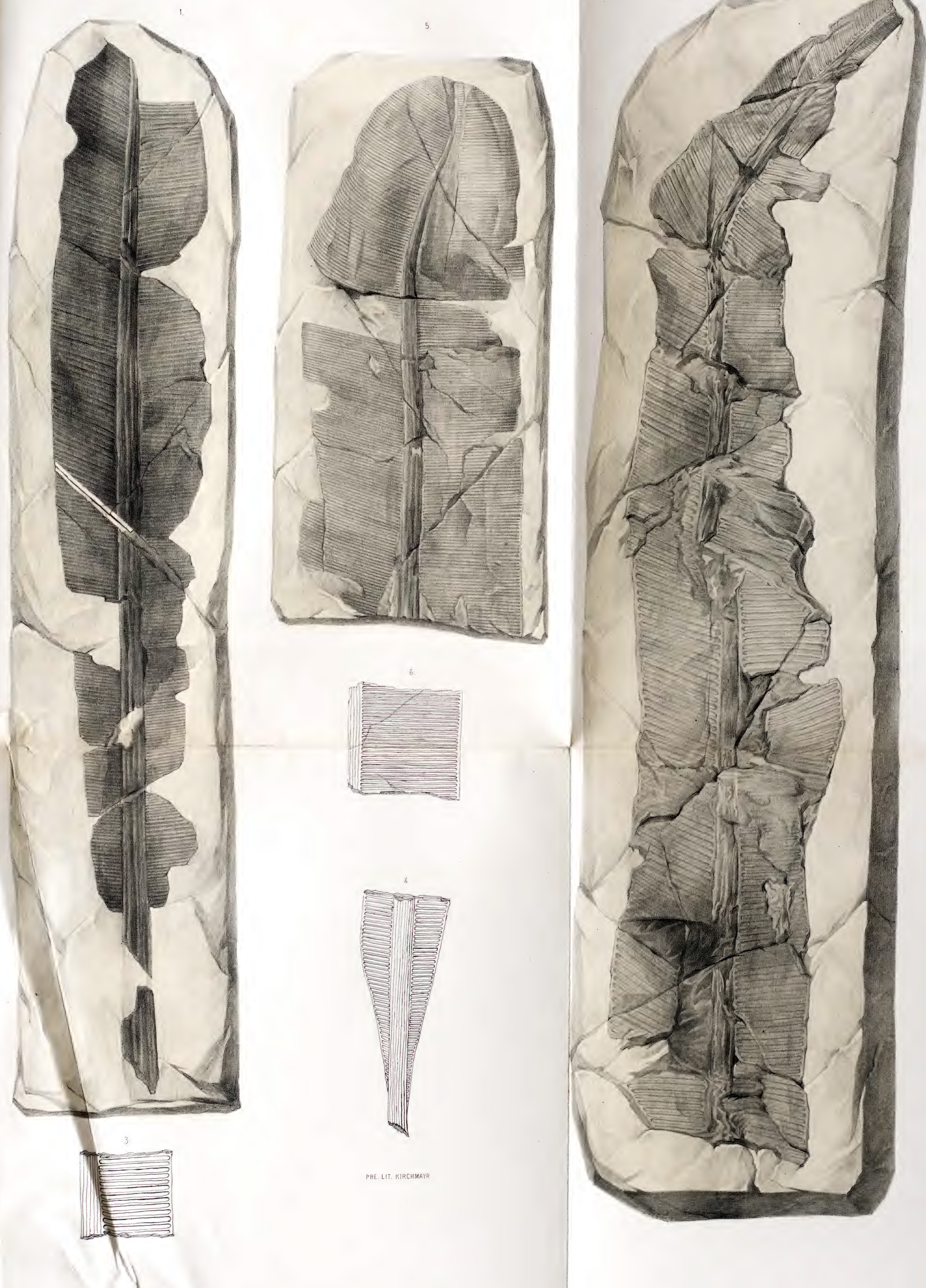




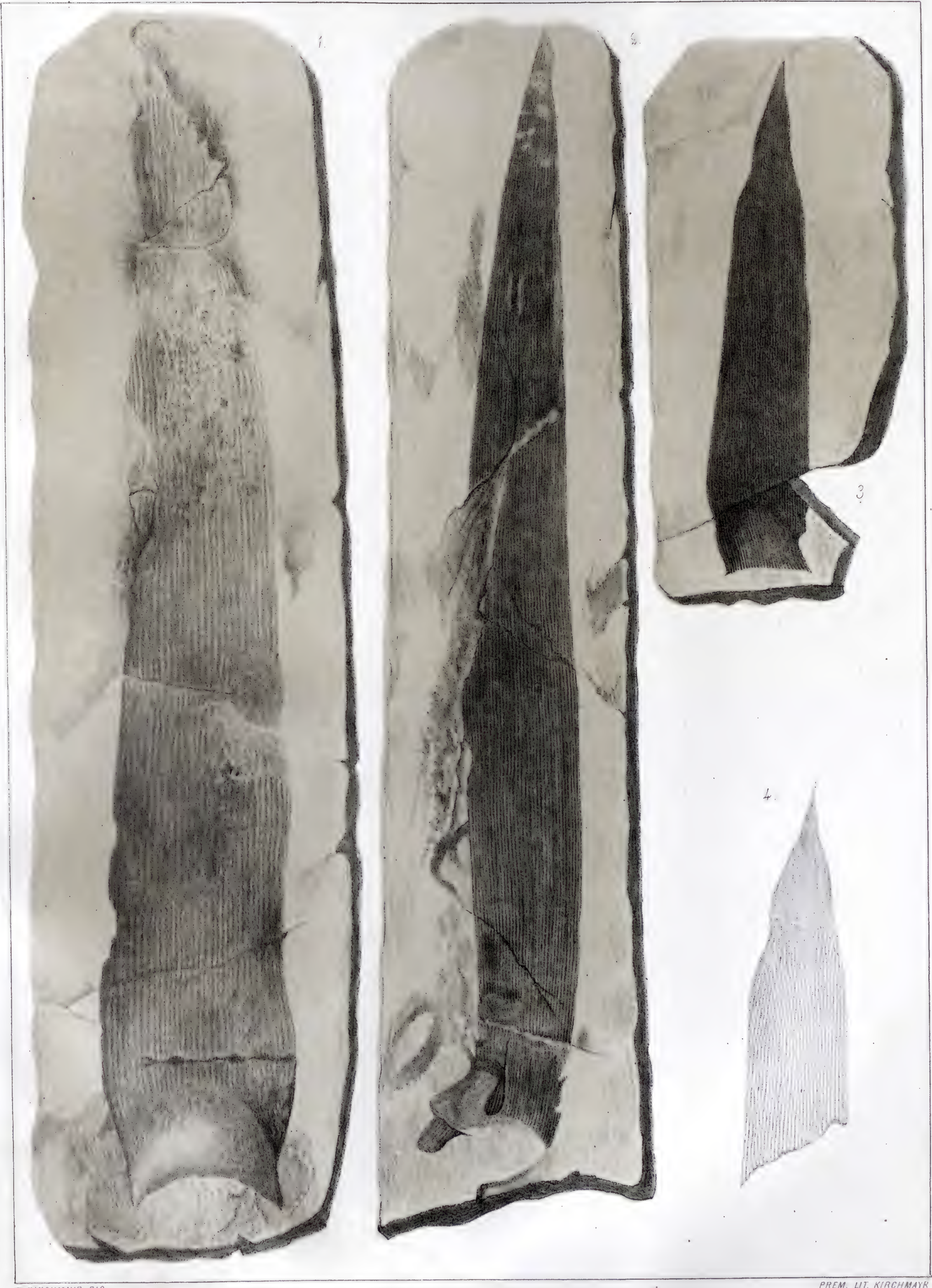




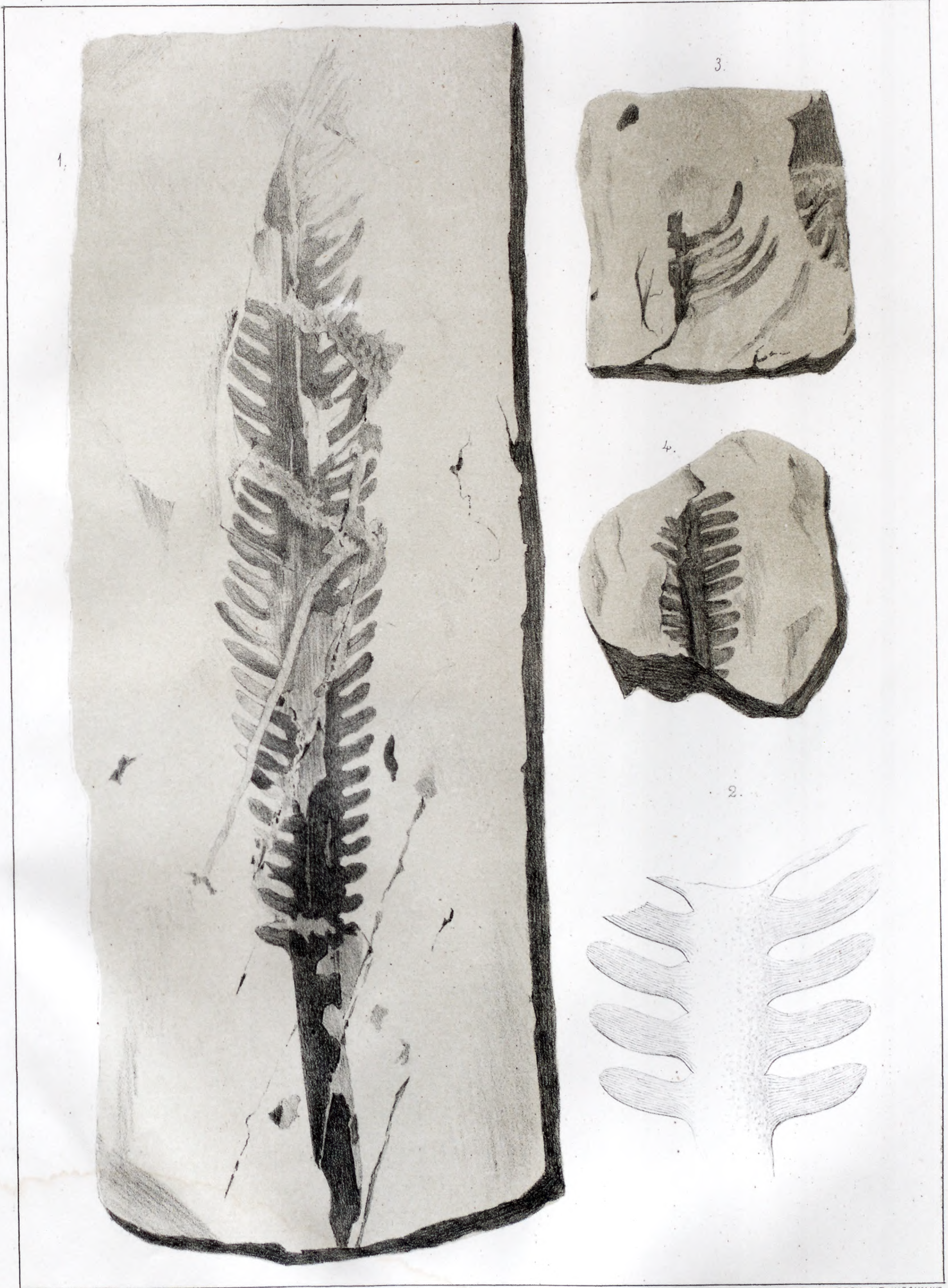



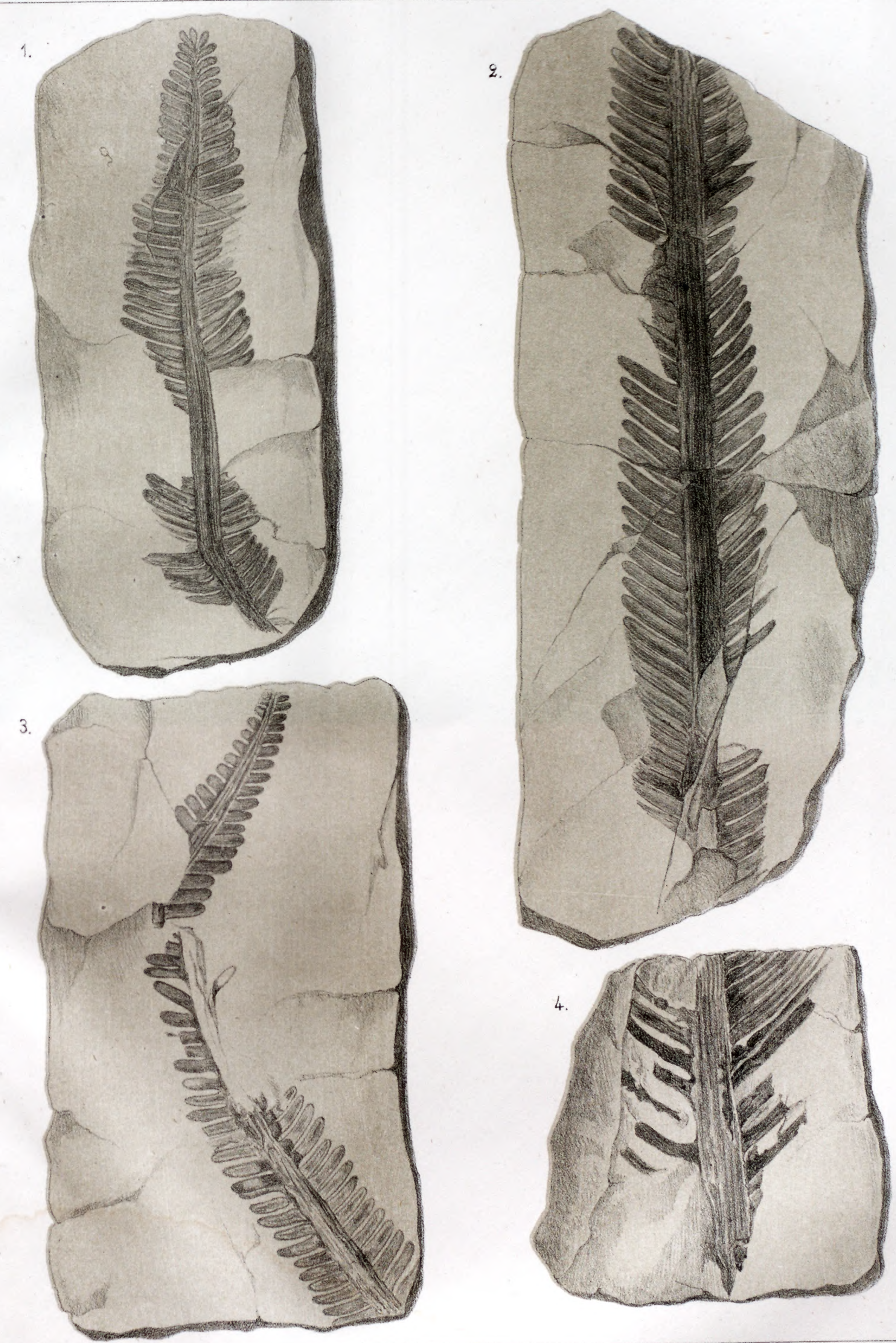


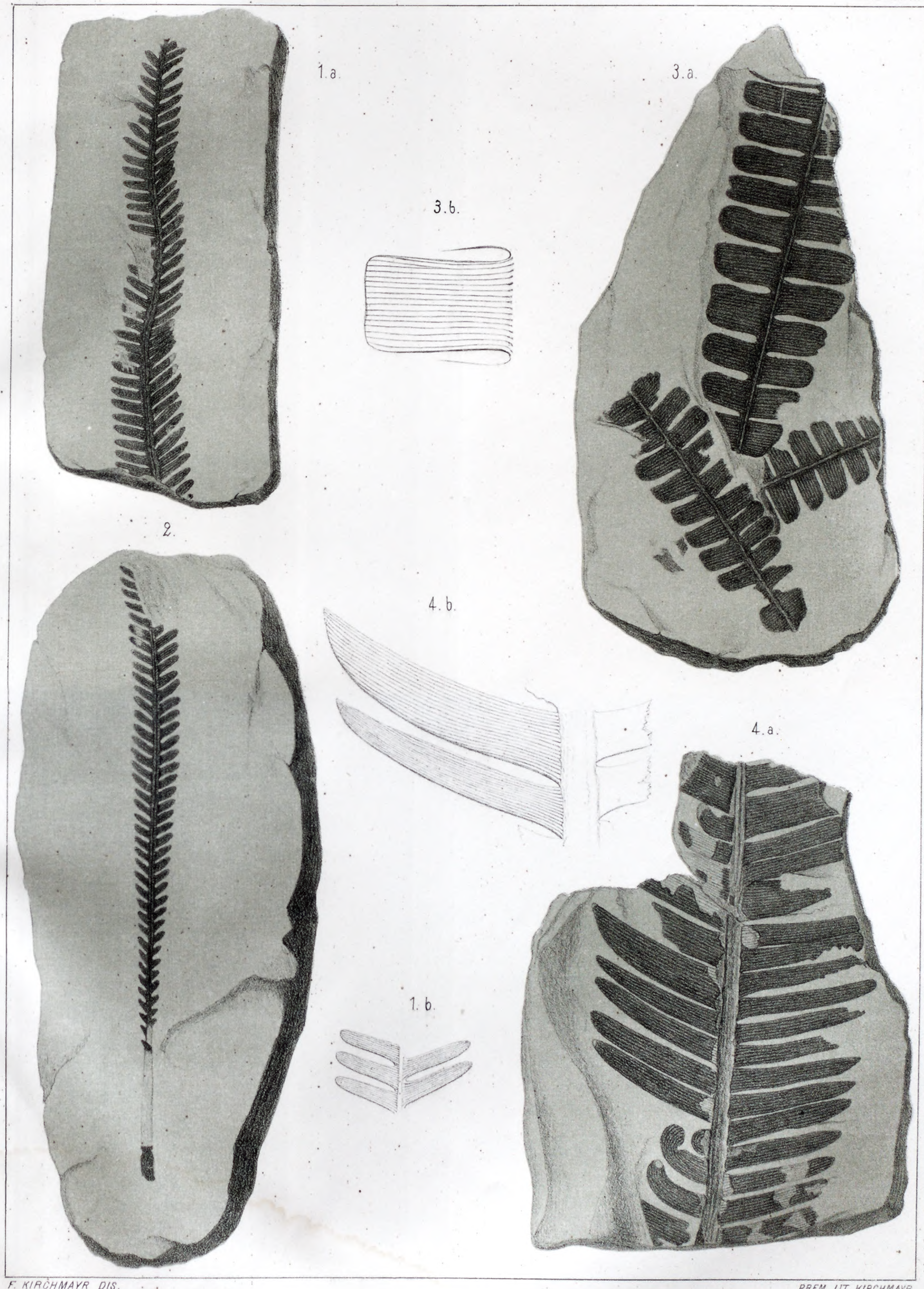


